\title{
New sample preparation techniques of macromolecular complexes for high resolution structure determination using cryo-EM
}

\author{
DISSERTATION \\ for the award of the degree \\ "Doctor rerum naturalium" (Dr. rer. nat.) \\ of the GEORG-AUGUST-UNIVERSITÄT GÖTTINGEN
}

within the doctoral program

MOLECULAR BIOLOGY

of the Göttingen Graduate Center for Molecular

Biosciences, Neurosciences and Biophysics (GGNB)

submitted by

Kashish Singh

from Jammu, India

Göttingen, 2019 



\section{Thesis Committee}

Prof. Dr. Holger Stark

Structural Dynamics, Max Planck Institute for Biophysical Chemistry

Prof. Dr. Kai Tittmann

Bioanalytics, Göttingen Center for Molecular Biosciences

Prof. Dr. Ralf Ficner

Molecular Structural Biology, Institute for Microbiology and Genetics

\section{Members of the Examination Board}

$1^{\text {st }}$ Referee: Prof. Dr. Holger Stark

Structural Dynamics, Max Planck Institute for Biophysical Chemistry

$2^{\text {nd }}$ Referee: Prof. Dr. Kai Tittmann

Bioanalytics, Göttingen Center for Molecular Biosciences

Prof. Dr. Ralf Ficner

Molecular Structural Biology, Institute for Microbiology and Genetics

Prof. Dr. Markus Zweckstetter

Structure determination of proteins using NMR, Max Planck Institute for Biophysical Chemistry

Dr. Alex Faesen

Biochemistry of Signal Dynamics, Max Planck Institute for Biophysical Chemistry

Dr. Alexander Stein

Membrane Protein Biochemistry, Max Planck Institute for Biophysical Chemistry

Date of oral examination: $5^{\text {th }}$ July, 2019 



\section{AFFIDAVIT}

I hereby declare that this dissertation with the title "New sample preparation techniques of macromolecular complexes for high resolution structure determination using cryo-EM" has been written independently and with no other aids or sources than quoted. This thesis (wholly or in part) has not been submitted elsewhere for any academic award or qualification.

Kashish Singh 



\section{Abstract}

Fatty acids are one of the most abundant lipids in the cell. Cells use them to build biological membranes, as energy reserves and as signaling molecules. Fatty acids are synthesized by a specialized protein machinery called the fatty acid synthase (FAS). Despite the crucial role fatty acids play in a cell, we still know surprising little about the structure and mechanism of action of fatty acid synthases. In fungi and mammals, fatty acid synthases are large multimeric protein complexes. During fatty acid synthesis, the growing fatty acid chain is thought to be shuttled by the acyl carrier protein domain to several enzyme active sites. To accomplish this, these proteins need to be dynamic. This, however, limits our ability to study their structure at high resolution. In this doctoral thesis, different biochemical methods were tested to reduce compositional and conformational heterogeneity of the fungal type I FAS. To address compositional heterogeneity, a mild chromatography-free purification strategy was established along with the removal of bound fatty acid intermediates from the complex. Gradient Fixation (GraFix), nanobody binding and addition of substrates were also tested for conformational stabilization of the complex. The improvement in compositional heterogeneity allowed structures of the FAS to be routinely

determined at resolutions of $3 \AA$, using both cryo-EM and X-ray crystallography. The structures determined during this thesis are the highest resolution structures of the FAS reported to date. Structural analysis revealed two conformational states of the FAS. The first, a non-rotated conformation, where the acyl carrier protein domain is localized at the ketosynthase domain, and a novel rotated conformation, where the acyl carrier protein is localized at the acetyl transferase domain. Along with this, a novel $\gamma$-subunit of the S.cerevisiae FAS was characterized. This is the first FAS binding protein identified in over five decades of FAS research. The $\gamma$-subunit spans a distance of $120 \AA$ inside the FAS cavity and interacts with four domains: enoylreductase, acyl carrier protein, malonyl/palmitoyl transferase and ketoreductase. In addition, the $\gamma$-subunit stabilizes the rotated FAS conformation and reduces the affinity of FAS for its substrates. Through its interactions, the $\gamma$-subunit directly hinders the binding of the malonyl-CoA and NADPH to the respective catalytic domains. These results provide a better understanding of the dynamics of the 
fungal type IFAS. The dependence of ACP domain location on the conformation of the FAS dome suggests that the movement of the ACP inside the FAS might not be completely stochastic as previously postulated. Furthermore, the ability of the $\gamma$-subunit to regulate FAS activity by inhibiting multiple active sites is unique and adds a new mechanism of FAS regulation in yeast. In the future, the knowledge obtained by studying the $\gamma$-subunit can be applied for designing inhibitors based on its structure. It also offers a nature made scaffold that can be exploited to incorporate natural and designed enzymatic activities absent from the FAS. The work performed in this thesis underscores the need to revisit essential protein machineries using new sample preparation methods and structural techniques for a more comprehensive understanding of how protein structure correlates with function. 


\section{Acknowledgements}

I would like to thank all the members of the AG Stark/Department of structural dynamics, Holger Stark, Ashwin Chari, Fabian Henneberg, Lukas Schulte, Karl Bertram, Benjamin Graf, David Haselbach, Stephanie Schell, Wen-Ti Liu, Suzan Gerwen, Cole Townsend, Uma Dakshinamoorthy, Sabrina Fiedler, Jan Erik Schliep, Georg Bunzel, Felix Lambrecht, Zhenwei Zhang, Niels Fischer, Mario Lüttich, Tobias Koske, Thomas Conrad, Prakash Dube, Claudia Fahlbusch, Gaby Heyne, Hossein Kohansal, Uwe Lücken, Alexey Matyash, Alexander Mehr, Juliane Moses, Elham Paknia, Dietmar Riedel, Dirk Wenzel, Frank Würriehausen, Ai Woon Yee, Gudrun Heim, and Jil Schrader for a very supportive and friendly atmosphere during my stay in the lab. I would also like to thank Kai Tittmann, Victor Sautner and Andreas linden with whom I collaborated over the past four years.

To Holger, I would like to thank you for giving me the opportunity to join your lab. It has been a wonderful experience. I have enjoyed greatly working with you and learning from you. Your passion and enthusiasm for science is inspiring.

To Ashwin, thank you for giving me the chance to work with you. I am very grateful for the substantial amount of time that you have devoted towards this project and towards me. I owe most of my lab training to you and I also want to thank you for being patient with me all these years.

To Ben, thank you for all your help in this project. I hope you unravel more mysteries the FAS has to offer.

To Steffi, thank you for helping me in the lab when I initially joined.

To David, thank you for teaching me how to do cryo-EM and for all your advice.

To Kai, I would like to thank you for being a part of my TAC. All your time and advice was immensely helpful in this project.

I would also like to thank Viktor Sautner for his help with the kinetic measurements.

I would like to thank Suzan for purifying the nanobody clones. 
To Andreas, thank you for all the mass spectrometry analysis that you performed over the years. It was a pleasure working with you.

To Cole, it is always fun to talk to you, be it about science or general lab related matters. Thank you for your critical review of this thesis.

A special thanks to Fabian, Lukas and Karl, the members of the best office at MPIBPC. I could not have asked for a better group of people to start my $\mathrm{PhD}$ with. When we started, we all were young and naive but during these past years we have transformed, now we are old and naive. I hope we always stay in touch.

To Ina, this PhD would not have been the same if I hadn't met you. Thank you for making this the best time of my life, yet;).

I devote this PhD thesis to my parents and my sister who have encouraged me in all my endeavors and were there whenever I needed them. 


\section{Table of Contents}

ABSTRACT ................................................................................................................................ VII

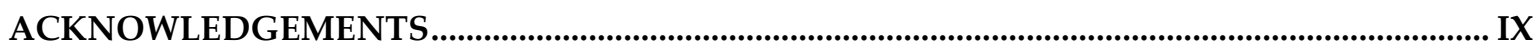

1. INTRODUCTION

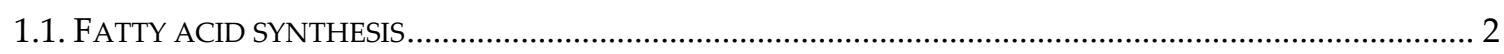

1.2. PROTEINS INVOLVED IN FATTY ACID SYNTHESIS....................................................................... 4

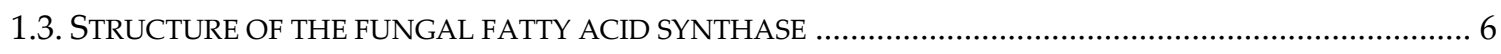

1.3.1. What is the structural basis behind substrate shuttling by ACP? ...................................... 8

1.3.2. Does the FAS structure change during substrate shuttling? ................................................. 9

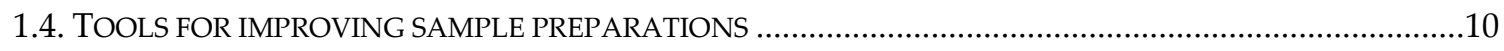

1.4.1. Chromatography-free purification ..................................................................................

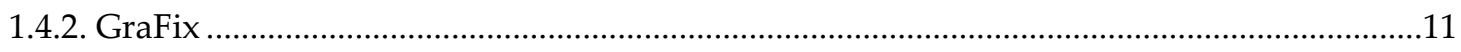

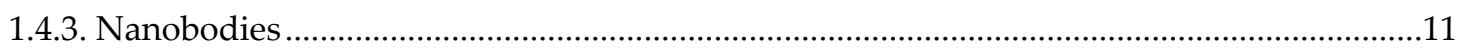

1.4.4. Substrates/Inhibitors....................................................................................................12

1.4.5. In-silico sorting and analysis of protein conformations ......................................................12

1.4.5.1 Conformational energy landscapes using 3D Principle Component Analysis (PCA) ................ 13

1.5. AIMS AND SCOPES - PART I ........................................................................................................14

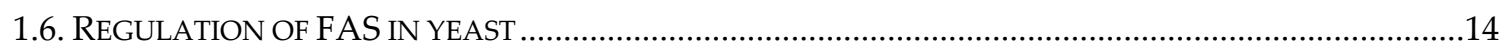

1.6.1. Transcriptional Regulation ............................................................................................

1.6.2. Translational regulation .................................................................................................. 15

1.6.3. Post-translational modification .............................................................................................

1.6.4. Post-translational degradation ............................................................................................ 16

1.6.5. Metabolite based regulation .........................................................................................17

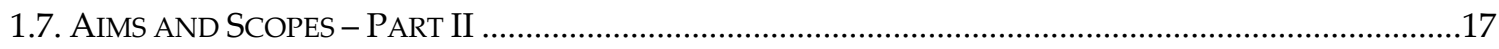

2. MATERIALS AND METHODS …………………..........................................................................18

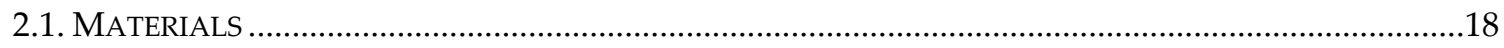

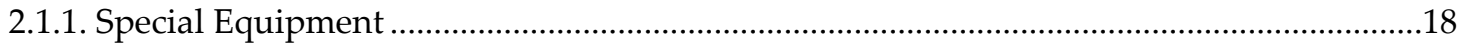

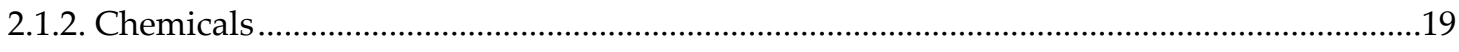

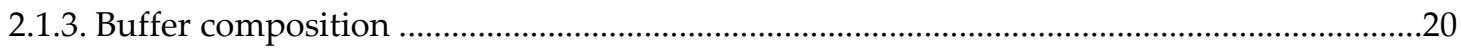

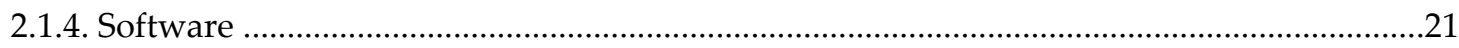

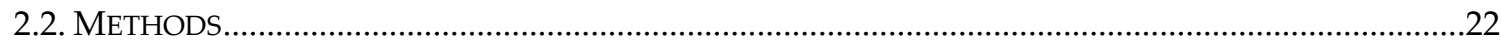




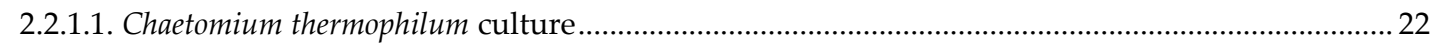

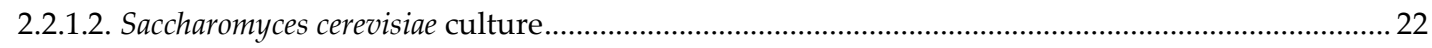

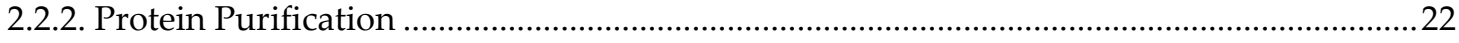

2.2.2.1. Purification of Chaetomium thermophilum FAS (CtFAS) ............................................................... 22

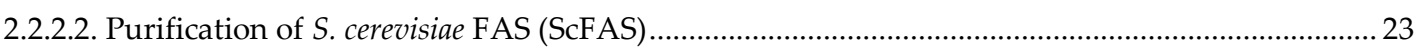

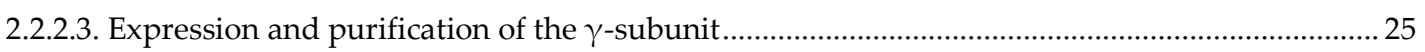

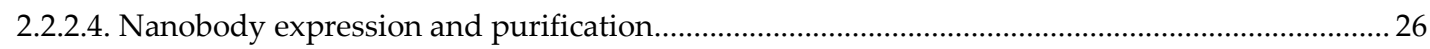

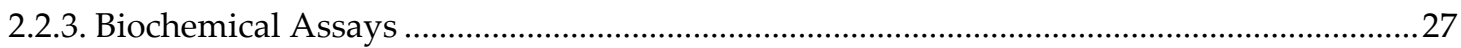

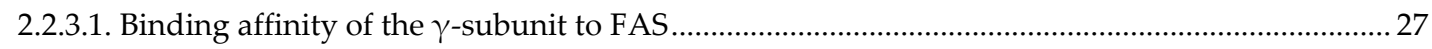

2.2.3.2. Salt concentration-dependent dissociation of the $\gamma$-subunit ..................................................... 27

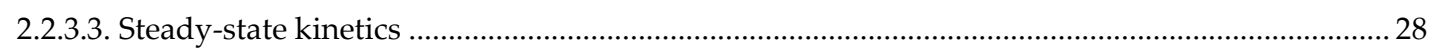

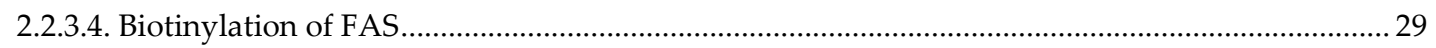

2.2.4. Crosslinking Mass Spectrometry ….................................................................................29

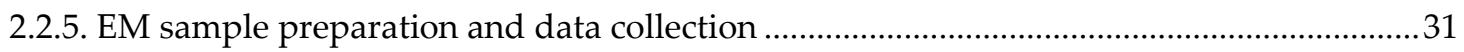

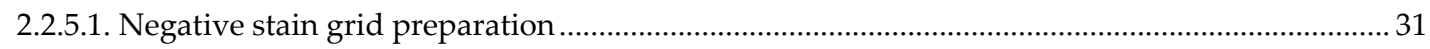

2.2.5.2. Glutaraldehyde concentration for CtFAS GraFix gradients ...................................................... 31

2.2.5.3. Preparative reconstitution of the FAS- $\gamma$-subunit complex …...................................................... 32

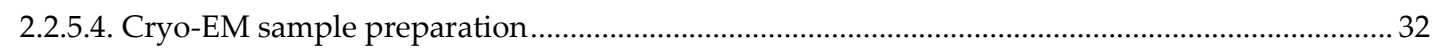

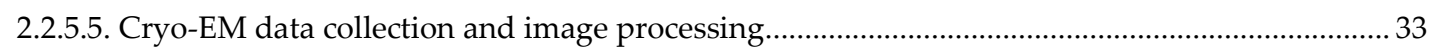

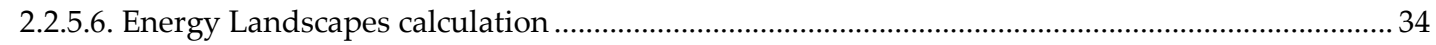

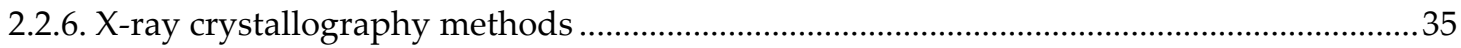

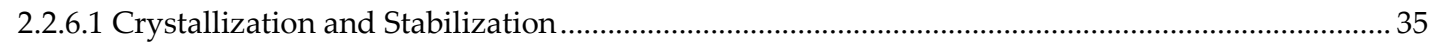

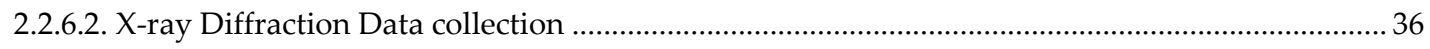

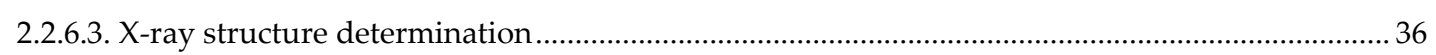

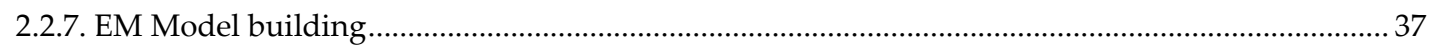

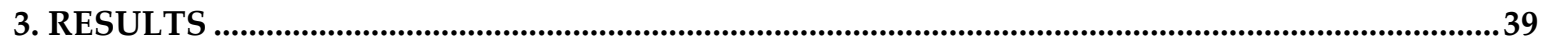

3.1. STRUCTURE INVESTIGATIONS OF CHAETOMIUM THERMOPHILUM FAS ..........................................39

3.1.1. Purification of endogenous FAS from Chaetomium thermophilum ...................................39

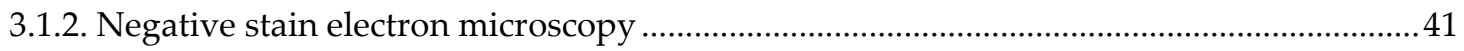

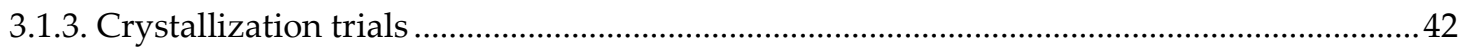

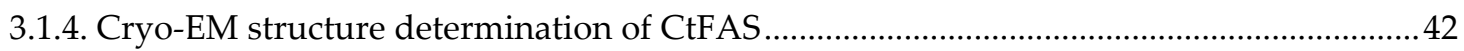

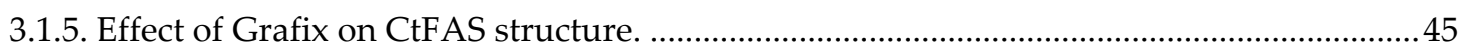

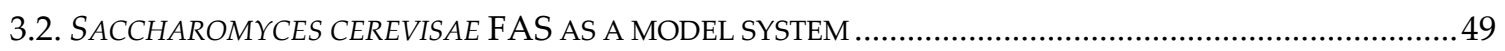

3.2.1. Purification of endogenous FAS from Saccharomyces cerevisiae .........................................49 
3.2.2. Discovery of a novel interactor of the yeast FAS .50

3.2.3. Mass spectrometric analysis of Tma17p and Tma17p-FAS complex................................54

3.2.4. Effect of FAS activity upon binding of Tma17p ..............................................................56

3.2.5 Molecular basis underlying the $\gamma$-subunit's inhibitory activity .......................................58

3.2.5.1. Cryo-EM analysis of endogenous FAS holoenzyme complex ..................................................... 59

3.2.5.3. Cryo-EM structure determination of FAS in the absence of the $\gamma$-subunit................................. 64

3.2.5.2. Crystallographic structure determination of FAS in the absence of the $\gamma$-subunit..................... 66

3.2.5.4. Cryo-EM structure of the reconstituted FAS holoenzyme complex ........................................ 70

3.2.5.5. Crystallographic model of the reconstituted FAS holoenzyme complex ................................... 72

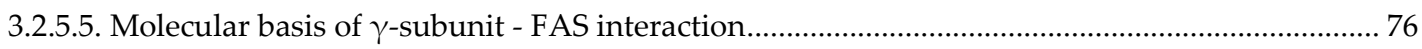

3.2.5.6. Changes in the conformational landscape of FAS due to the $\gamma$-subunit ........................80

3.3. OTHER TOOLS TESTED FOR STABILIZING THE FAS STRUCTURE ....................................................83

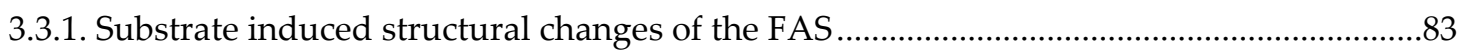

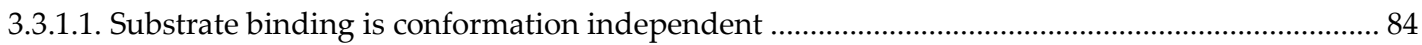

3.3.1.2. Local changes in FAS domains upon substrate binding ............................................................. 84

3.3.1.3. Effect of dome rotation on NADPH binding to the ER domain ............................................... 86

3.3.1.4. Comparison between the FAS holoenzyme complex and the rotated FAS conformation in the

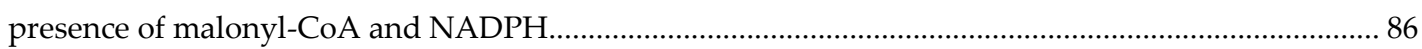

3.3.2. Conformational stabilization using anti-FAS nanobodies .............................................87

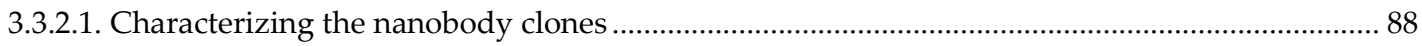

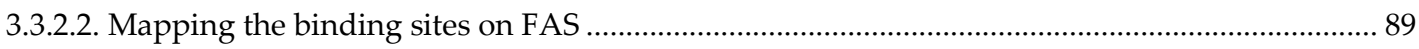

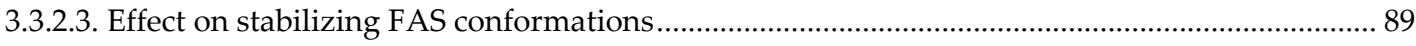

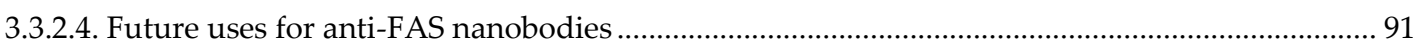

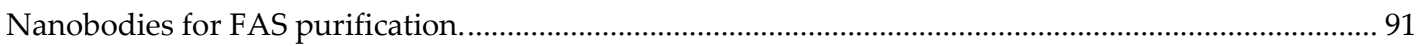

4. DISCUSSION

4.1. OBTAINING STABLE MACROMOLECULAR COMPLEXES FOR STRUCTURAL STUDIES ..........................93

4.1.1. Purification of compositionally homogeneous proteins..................................................94

4.1.2. Thermodynamic trapping of protein complexes ............................................................94

4.1.2.1. Working at $4{ }^{\circ} \mathrm{C}$ to populate energetic conformational minima.................................................. 94

4.1.2.2. Thermophilic vs mesophilic organisms as a source for protein complexes .............................. 95

4.1.3. Using protein function for conformational stabilization ...............................................95

4.1.4. Using GraFix based chemical fixation...........................................................................96

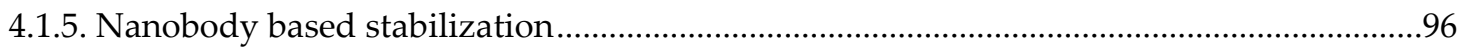

4.2. STRUCTURAL METHODS FOR STUDYING LARGE MULTIDOMAIN COMPLEXES .................................97

4.2.1. Studying protein structure in solution or in a crystalline lattice? ...................................97 
4.2.2. Cryo-EM maps vs crystallographic maps for model building

4.2.3. 3D PCA - energy landscapes as a tool for analyzing cryo-EM data ....................................99

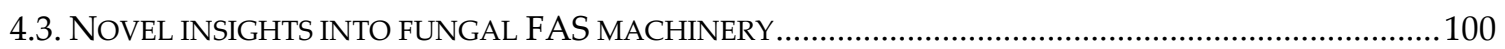

4.3.1. Conformations of the FAS dome correlate with ACP movement .....................................100

4.3.2. Conformational changes provide directionality to ACP movement ....................................100

4.3.3 Tma17p - a novel $\gamma$-subunit of the yeast FAS …………………………………………....101

4.3.4. Is the $\gamma$-subunit involved in different cellular processes? ..................................................103

4.2.2 Present hypothesis for the role of $\gamma$-subunit in the cell ................................................. 104

4.2.4. $\gamma$-subunit is conserved among yeast but not in higher eukaryotes .....................................105

4.2.5. Biotechnological applications of the FAS and its $\gamma$-subunit ..............................................106

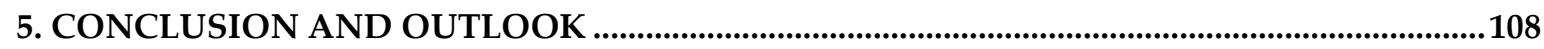

ABBREVIATIONS ..........................................................................................................................110

SUPPLEMENTARY INFORMATION ...............................................................................................112

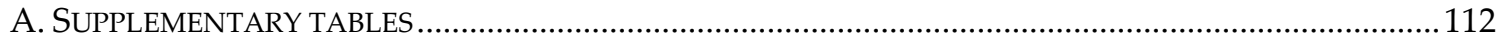

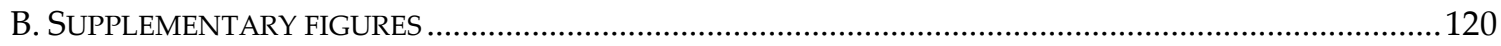

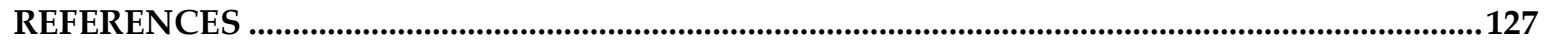

CURRICULUM VITAE .......................................................................................................................137 


\section{Introduction}

Proteins are one of the most abundant biological macromolecules in cells and have a diverse range of functions. Proteins can be regulatory, structural, protective, serve in transport, signaling, storage, or act as biological catalysts. They also enable synthesis and degradation of all other types of biological macromolecules. The ability of proteins to perform such diverse functions comes from the diversity of their structures. Building units of proteins are called amino acids. There are 21 naturally occurring amino acids, which can be joint linearly to form chains of varying lengths. Linear chains of amino acids further fold in 3D space. Given that any number of amino acids can arrange in any given order, it becomes clear that the combinatorial potential that exists for building proteins is virtually indefinite. What is more, individual proteins can further combine with other proteins, but also carbohydrates, lipids or nucleic acids to form large macromolecular complexes. Aside from their structural complexity, macromolecular complexes are dynamic and can undergo changes in composition and 3D conformation. Simply put, protein sequence determines its 3D architecture and its 3D architecture determines its function. Therefore, to understand the different cellular processes, it is important to study the 3D structures of the complexes driving them.

One such large macromolecular complex is the fatty acid synthase. Cells rely on this protein machinery to produce fatty acids, one of the most abundant lipids in the cell. Fatty acids are carboxylic acids with long aliphatic chains which are essential for cellular function. Cells use fatty acids to build biological membranes in the form of phospholipids and glycolipid, as energy reserves in the form of triacylglycerol, and as signaling molecules in the form of phosphatidylinositol and sphingosine derivatives (Wang et al., 2011). Despite the crucial role fatty acids play in a cell, we still know surprising little about the structure and mechanism of action of fatty acid synthases. In fungi and mammals, fatty acid synthases are large multimeric protein complexes (Lynen, 1980; Smith et al., 2003). These complexes contain all catalytic activities for de novo synthesis of fatty acids. During fatty acid synthesis, the growing fatty acid chain is thought to be shuttled by their acyl carrier protein domain to the several enzyme active sites (Chan and Vogel, 2010; Herbst et al., 2018; Schweizer and 
Hofmann, 2004). To accomplish the structural rearrangements required for their function, these proteins need to be very dynamic. This, however, limits our ability to study their structure at atomic resolution.

Presently, X-ray crystallography and single particle cryo-electron microscopy (cryo-EM) allow us to study large macromolecular complexes at high resolutions (Cheng, 2015; Shi, 2014). These techniques rely on isolated proteins for obtaining structural information. The purified proteins need to be devoid of impurities. Likewise, they should be compositionally and conformationally homogenous. Obtaining such samples becomes difficult as the size and complexity of the protein complexes increase. Purification strategies involving the use of high salt concentrations along with use of chromatography steps, such as anion exchange and size exclusion work well for small $(<100 \mathrm{kDa})$ proteins, but can have detrimental effects on larger protein complexes (Liu et al., 2001). Multi-protein complexes tend to dissociate under high ionic strength. Along with this, shear forces due to interactions with resin used in chromatography columns can cause the loss of bound subunits (Tsumoto et al., 2007). Adding to this complexity, large protein complexes are also inherently dynamic and can exist in multiple conformations (Stark and Chari, 2016). Alternative purification methods and methods to stabilize large multimeric protein complexes are therefore necessary for studying their structure at high resolutions.

In the following chapters, I review the current structural knowledge of the fungal FAS. This will be followed by a description of the tools for improving sample preparations of large macromolecular complexes that were used in this thesis. Finally, I will briefly summarize the current knowledge regarding regulation of fungal FAS.

\subsection{Fatty acid synthesis}

Organisms such as bacteria, fungi and plants depend solely on de novo cellular synthesis of fatty acids to meet their needs (Brown et al., 2009; Janßen and Steinbüchel, 2014; Schweizer and Hofmann, 2004). Vertebrates, on the other hand, cannot synthesize all required fatty acids and must therefore rely on dietary fatty acids such as omega- 3 and omega- 6 fatty acids (Chirala et al., 1997; Di Pasquale, 2009; Swanson et al., 2012). The biochemical 
mechanisms underlying fatty acid synthesis, however, are conserved from bacteria to humans (Lynen, 1980; Schweizer and Hofmann, 2004; Wakil et al., 1983). It requires three substrates - acetyl-CoA, malonyl-CoA and NAPDH (or NADH). Fatty acid synthesis is an iterative process where for each fatty acid chain, the first two carbon atoms come from an acetyl-CoA molecule and the rest come from malonyl-CoA (Figure 1).

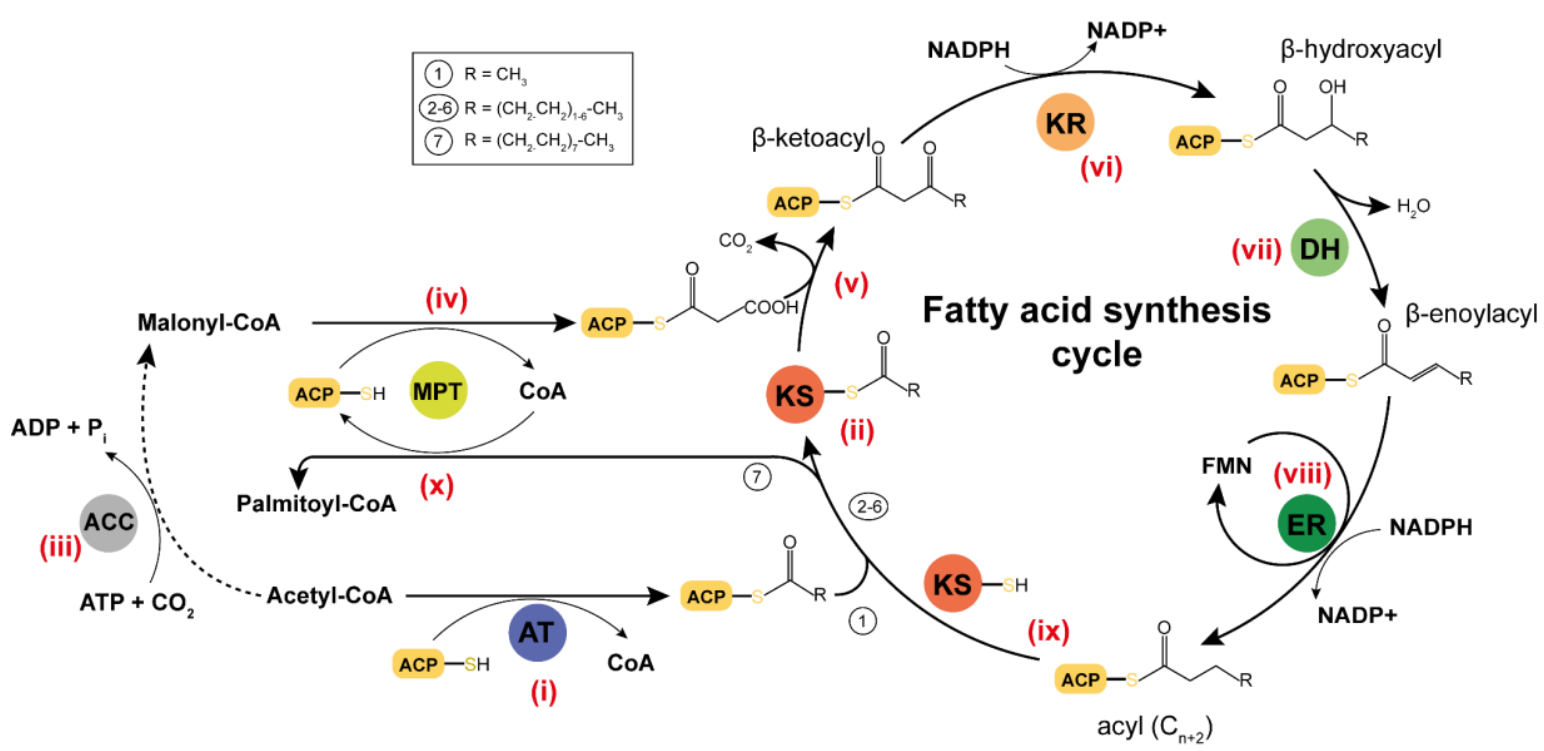

Figure 1. Fatty acid synthesis cycle in fungi. Fatty acid synthesis is an iterative process. (i) It starts with the transfer of an acetyl group from acetyl-CoA to an acyl carrier protein (ACP). (ii) The acetyl-ACP then is transferred to the active site thiol of the ketosynthase (KS) active site. (iii) Next, malonyl-CoA is synthesized from acetyl-CoA by acetyl-CoA carboxylase (ACC). (iv) Malonyl group from the malonyl-CoA is transferred to an ACP by the malonyl/palmitoyl transferase (MPT). (v) The KS domain bound acetyl group undergoes a condensation reaction with malonyl- $A C P$ in the KS active site to form $\beta$-ketobutyryl-ACP. (vi) The $\beta$-ketobutyryl-ACP is then reduced by the ketoreductase enzyme (KR) using reducing equivalents from a $N A D(P) H$ molecule to form $\beta$ hydroxybuturyl-ACP. (vii) The dehydratase enzyme (DH) then catalyzes a dehydration reaction to form $\alpha, \beta$-trans-butenoyl-ACP. (viii) The enoylreductase (ER) enzyme uses reducing equivalents from another $N A D(P) H$ molecule to reduce the unsaturated bond of $\alpha, \beta$-trans-butenoyl-ACP to form butyryl-ACP. This ends the first synthesis cycle. (ix) In the next cycle, the butyryl moiety is transferred to the KS active site thiol followed by another condensation reaction with the malonyl$A C P .(x)$ The cycle repeats 6 more times after which the palmitoyl group from the palmitoyl-ACP is transferred onto a free CoA molecule by the MPT domain. Based on Herbst et al. (2018).

The biosynthesis pathway starts with the transfer of an acetyl group from an acetyl-CoA onto a phosphopantetheine prosthetic (Ppant) group of the acyl carrier protein (ACP). The 
acetyl-ACP is then transferred to the active site thiol of the ketosynthase (KS) active site. Next, malonyl-CoA synthesized by acetyl-CoA carboxylase (ACC) is used to extend the two carbon atoms provided by acetyl CoA. Malonyl group from the malonyl-CoA is transferred to an ACP by the malonyl/palmitoyl transferase (MPT). The acetyl group then undergoes a condensation reaction with the malonyl-ACP in the KS active site to form $\beta$-ketobutyryl$\mathrm{ACP}$. The $\beta$-ketobutyryl-ACP is then reduced by the ketoreductase enzyme (KR) using reducing equivalents from a $\mathrm{NAD}(\mathrm{P}) \mathrm{H}$ molecule to form $\beta$-hydroxybuturyl-ACP. The $\beta$ hydroxybuturyl-ACP in turn is acted on by the dehydratase enzyme (DH) resulting in removal of a water molecule forming $\alpha, \beta$-trans-butenoyl-ACP. Finally, the enoylreductase (ER) enzyme uses reducing equivalents from another $\mathrm{NAD}(\mathrm{P}) \mathrm{H}$ molecule to reduce the unsaturated bond of the $\alpha, \beta$-trans-butenoyl-ACP to form butyryl-ACP. This ends the first synthesis cycle. In the next cycle, the butyryl moiety is transferred to the KS active site thiol followed by another condensation reaction with the malonyl-ACP. The cycle then continues as mentioned above and is repeated until the formation of C16/18 long aliphatic chain which is transferred to a free CoA molecule by the MPT domain.

\subsection{Proteins involved in fatty acid synthesis}

The different steps of de novo fatty acid synthesis require seven different types of modules: six enzymes and an ACP domain to shuttle the substrates as well as intermediates among them. The arrangement of these seven modules varies among bacteria, fungi and mammals (Beld et al., 2015). In bacteria, plants, as well as eukaryotic organelles, such as mitochondria and plastids, each activity is located on separate polypeptides. This is referred to as the type II FAS system (White et al., 2005). Here, the proteins rely on random interactions in the cytosol, which makes the type II FAS very inefficient. To compensate for this, bacteria usually have high cytosolic concentrations of ACP $(\sim 100 \mu \mathrm{M})$ to increase the probability of ACP interacting with the right enzyme (Davis et al., 2001; Tang et al., 1997). However, as the ACP with the bound intermediates are exposed to all surrounding proteins in the cytosol, this arrangement is helpful in shuttling intermediates to downstream reactions, i.e. for synthesis of unsaturated fatty acids, or other secondary metabolites (White et al., 2005). In fungi, all modules are present on either one or two polypeptide chains, classified as the 
fungal type I FAS. Here the proteins form a large barrel-shaped multimeric complex, which contains all activities required for fatty acid synthesis. It usually has an $\alpha_{6} \beta_{6}$ heterododecameric arrangement with molecular weights ranging between 2-3 MDa (Schweizer and Hofmann, 2004). The proximity of the active sites in this arrangement makes these enzymes more efficient than the bacterial type I system. For example, based on the dimensions of the fungal FAS, the local concentration of ACP is calculated to be $\sim 1 \mathrm{mM}$ (Jenni et al., 2007). This is 10 times higher than in bacteria, allowing for faster transfer of intermediates between the different active sites. The last type of FAS assembly is the mammalian type I FAS. In this system, all the activities are present on a single $270 \mathrm{kDa}$ polypeptide, which forms a ' $X$ '-shaped, $\alpha 2$-dimeric complex (Maier et al., 2008; Smith et al., 2003). This arrangement merges the benefits of both the bacterial and fungal FAS systems. Here, the enzymatic domains are in close proximity, but the overall architecture allows the $\mathrm{ACP}$ to interact with other cytosolic proteins.

(i)

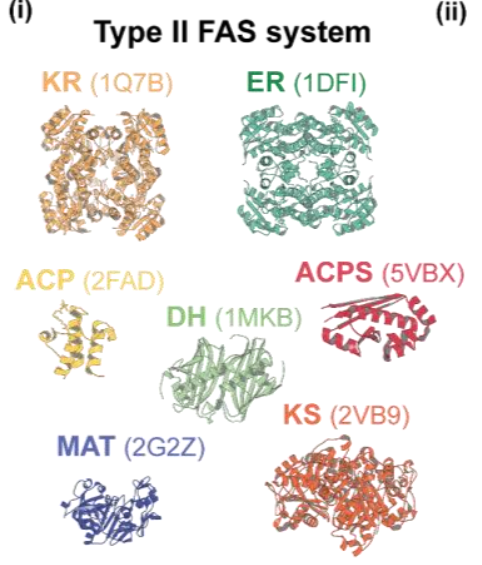

(ii)

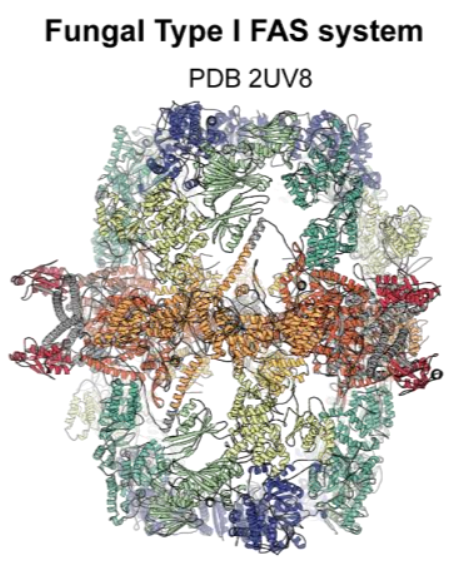

(iii)

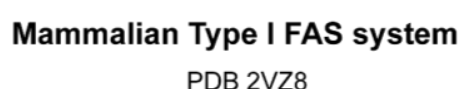

PDB 2VZ8

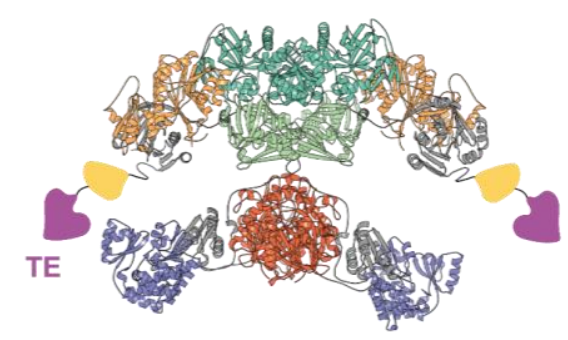

Figure 2. Organization of modules required for fatty acid synthesis. (i) In bacteria, plants and eukaryotic organelles, the modules required for fatty acid synthesis are present on separate polypeptides. This is referred to as the type II FAS. Structures of the ketoreductase (KR), enoylreductase (ER), acyl carrier protein (ACP), dehydratase (DH), ACP synthase (ACPS), malonyl-lacetyl-transferase (AT) and ketosynthase (KS) enzymes are shown with their corresponding PDB ID within parenthesis. (ii) In Fungi, all the modules shown in (i) are present on a single or two polypeptide chains which assemble into large "barrel-shaped" multi-subunit complexes. This is known as the fungal FAS type I. (iii) In mammals, all the modules are present on a $270 \mathrm{kDa}$ polypeptide, which forms a "X-shaped" dimeric complex. The thioesterase (TE) and ACP (yellow) domains were not visualized in the crystal structure and are depicted as cartoons. This figure is adapted from Beld et al. (2015). 


\subsection{Structure of the fungal fatty acid synthase}

Most fungal FAS are composed of two subunits - $\alpha$ and $\beta$. Together they form a $2.6 \mathrm{MDa}$, $\alpha_{6} \beta_{6}$ heterododecameric complex. The complex has a barrel-like structure, which is $270 \AA$ long and $260 \AA$ wide (Jenni et al., 2007; Leibundgut et al., 2007; Lomakin et al., 2007). The $\alpha$-subunit has four different domains: acyl carrier protein (ACP), ketosynthase (KS), ketoreductase (KR) and phosphopantetheine transferase (PPT). The $\beta$-subunit also has four domains: acetyl transferase (AT), enoyl reductase (ER), dehydratase (DH) and malonyl/palmitoyl transferase (MPT). Additionally, the first 94 residues of the $\alpha$-subunit also contribute towards the formation of the MPT domain. Six $\alpha$-subunits combine to form the central wheel of the molecule, whereas three $\beta$-subunits combine to form a dome on each side of the central wheel. In this arrangement, each dome contains three sets of active sites required for fatty acid synthesis along with three ACP domains. Each ACP domain is located within the barrel of the FAS where it is tethered to the MPT domain at its Nterminus and to the central wheel at its C-terminus through flexible linkers. During fatty acid synthesis, the ACP domains are responsible for shuttling the substrates and acyl intermediates from one active site to the other within each dome of the FAS.

The present structural view of the fungal FAS is a result of work performed by multiple labs. The first high resolution FAS structure from Thermomyces lanuginosus at $3.1 \AA$ allowed the placement of all domains except the ACP and PPT (Jenni et al., 2007). The ACP domain was then visualized for the first time in the Saccharomyces cerevisiae FAS at $3.1 \AA$ (Leibundgut et al., 2007). Here, the ACP was found to be located at the central wheel with its phosphopantetheine group protruding into the KS active site cleft. However, the protein sequences tethering the ACP to the FAS barrel were found to be flexible and therefore not visualized. The last unresolved domain of the FAS, PPT, was solved using the crystal structure of the isolated PPT domain at $2.9 \AA$ (Johansson et al., 2009). 

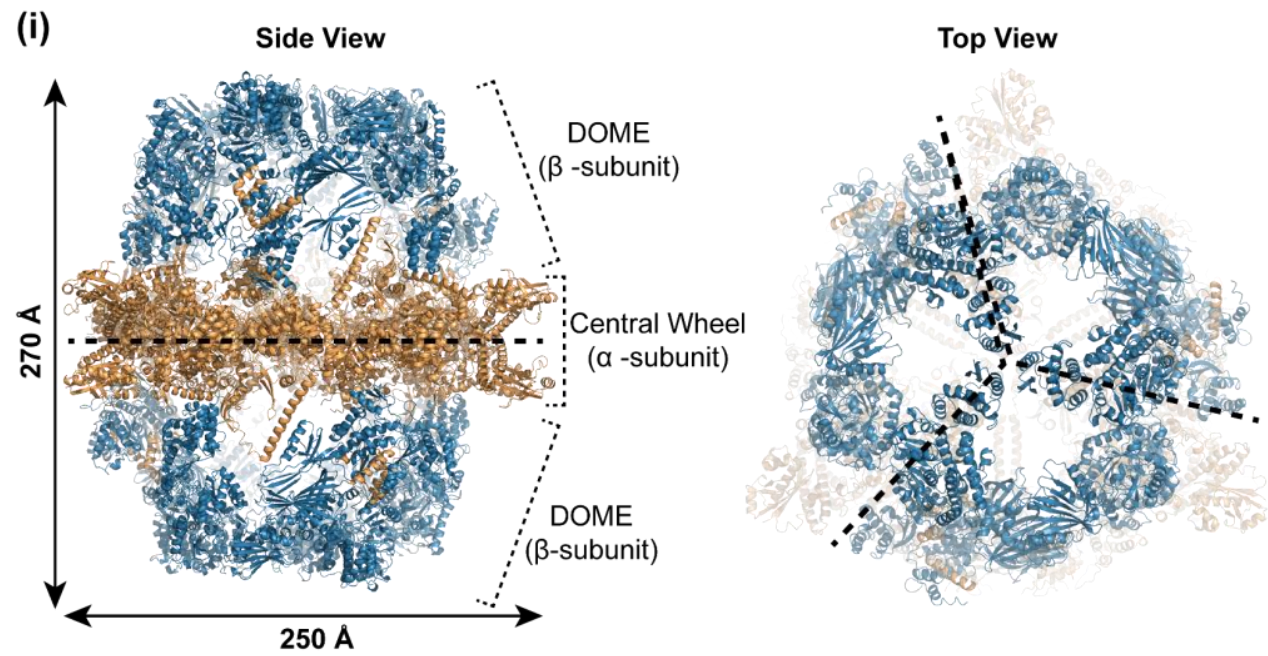

(ii)
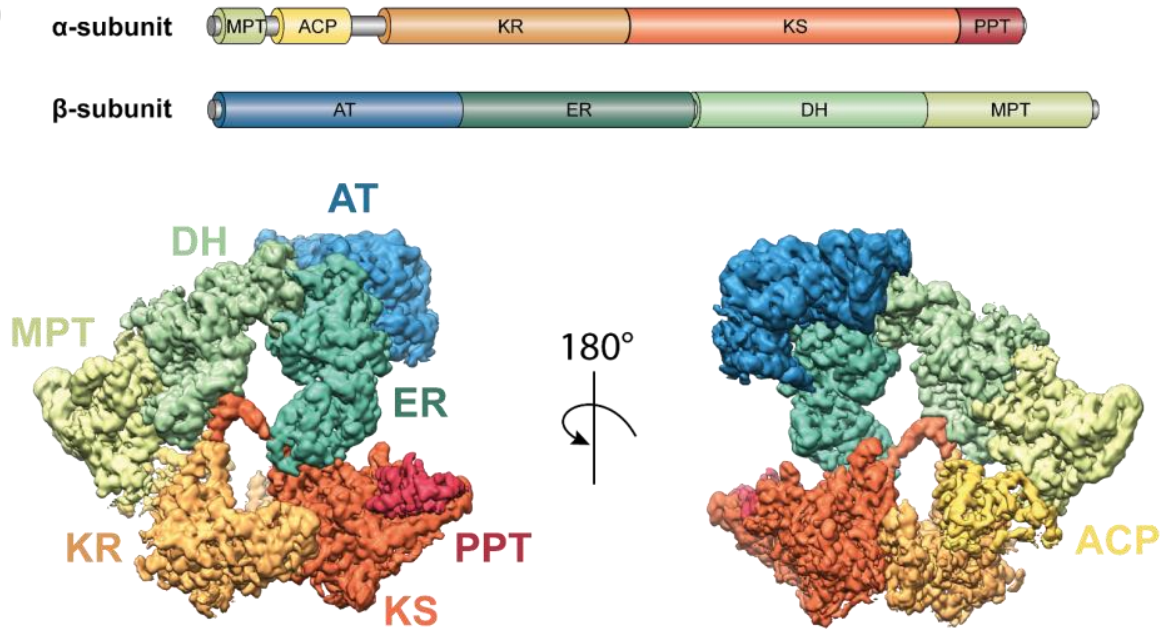

Figure 3: Architecture of the fungal fatty acid synthase. (i) The fungal FAS is composed of two subunits, $\alpha$ and $\beta$. Together the two subunits form a "barrel" like structure which is $270 \AA$ long and $260 \AA$ wide. The $\alpha$-subunits form the central wheel of the complex whereas the $\beta$-subunits form the dome of the complex on each side of the central wheel. The FAS has a D3 symmetry; a two-fold symmetry axis dissects the central wheel perpendicularly, whereas the three-fold axis runs down the length of the molecule (dotted line) (ii) The domain organization and architecture of the asymmetric unit of the FAS is shown. A small segment of the malonyl/palmitoyl-CoA acyl transferase (MPT) domain along with acyl carrier protein $(A C P)$, ketosynthase $(K S)$, ketoreductase $(K R)$ and phosphopantetheine transferase (PPT) domains reside on the $\alpha$-subunit. The acetyl transferase (AT), enoylreductase (ER), dehydratase (DH) and malonyl/palmitoyl-CoA acyl transferase (MPT) reside on the $\beta$-subunit. 
Even though the complete structure of the FAS is known since 2010, there are still two important questions regarding the functioning of this complex that remain unanswered: 1 ) what is the structural basis behind substrate shuttling by ACP and 2) does the structure of the FAS change during substrate shuttling?

\subsubsection{What is the structural basis behind substrate shuttling by ACP?}

The molecular basis behind ACP shuttling in fungal FAS is poorly understood. The model for the determinants driving this process comes from molecular dynamic simulations (Anselmi et al., 2010). This study provided three insights. First, the ACP binding to different domains is dependent on electrostatic interactions. Second, the individual ACP domains can access all the catalytic sites in a reaction chamber, but their movement tends to be compartmentalized due to volume-exclusion effects by other ACP domains and their linkers. Third, even though the ACP moves randomly in 3D, most domains are localized in a circular path such that the adjacent domains follow the same order as required for synthesis. This domain arrangement promotes a more efficient and productive transfer of the ACP domain from one site to the other as compared to random diffusion. However, no direct biochemical or structural evidence supporting these simulations have been reported until date. The only structural evidence for substrate shuttling present is through cryo-EM analysis of cerulenin inhibited S. cerevisiae FAS (Gipson et al., 2010). As compared to the FAS crystal structures (Johansson et al., 2008; Leibundgut et al., 2007) where the ACP domain was found adjacent to the KS domain, the $5.9 \AA$ cryo-EM structure displayed fragmented densities corresponding to the ACP domain next to all catalytic domains inside the dome. Even though this structure shows that the ACP domain can be located at different catalytic domains, it fails to provide mechanistic details regarding inter-domain interactions. The low-resolution and the presence of ACP domain densities next to all catalytic domains in one structure suggests conformational heterogeneity. The presence of more than six ACP domain densities is only possible when multiple conformations are averaged into one structure. To improve upon these observations, high resolutions structures of conformationally homogenous FAS molecules are required. For this, all ACP domains need to be stalled at the same site before structural studies are performed. Such 
structures of the FAS with defined ACP positions would aid in understanding the ACP shuttling process.
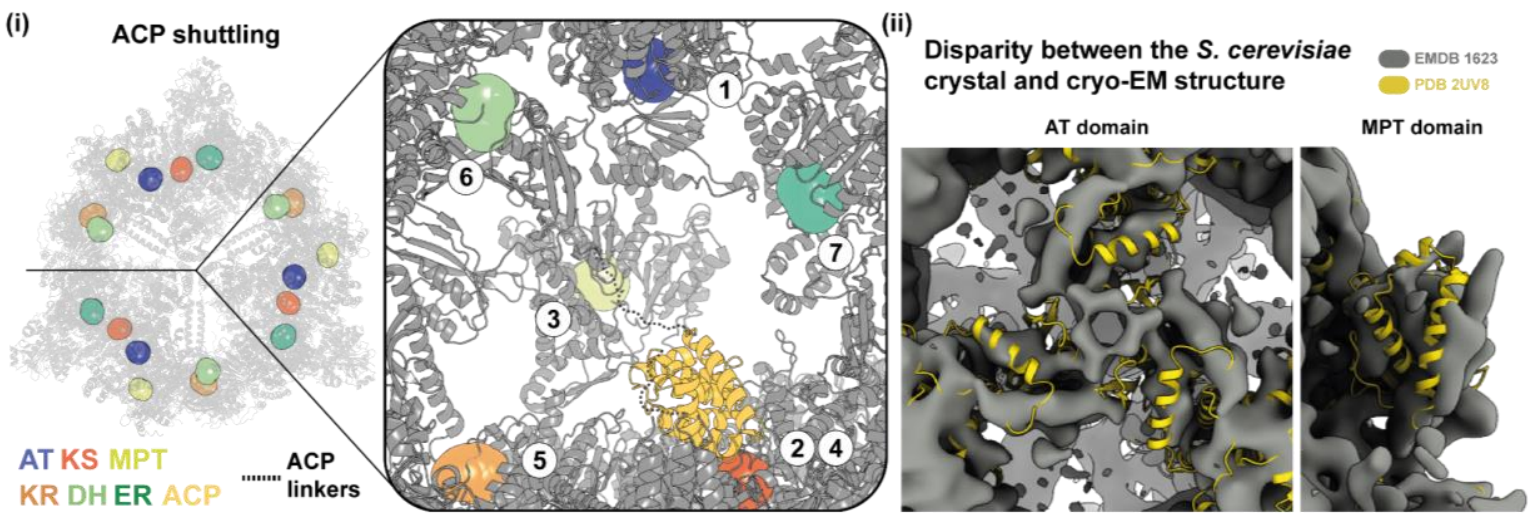

Figure 4. (i) Substrate shuttling by the ACP domain. Each FAS dome has three sets of active sites required for fatty acid synthesis. Additionally, the ACP (golden) is located within the FAS dome and is attached to the FAS through flexible linker sequences (black dotted line). During the catalytic cycle, the ACP domain shuttles the substrates and reaction intermediates among the six different catalytic domains. The numbers indicate the sequence of ACP movement during the first cycle of fatty acid synthesis. (ii) Structural difference between the S. cerevisiae FAS crystal structure and cryo-EM map. Overlay of the AT and MPT domain segments from the S. cerevisiae FAS crystal structure (yellow, PDB 2UV8, Leibundgut et al. (2007)) with the cryo-EM map (grey, EMDB 1623, Gipson et al. (2010)).

\subsubsection{Does the FAS structure change during substrate shuttling?}

Along with the presence of ACP densities next to the catalytic domains, the S.cerevisiae cryoEM structure (Gipson et al., 2010) also reported to have a different conformation of the dome in comparison to the crystal structures. The dome was more compact due to a rearrangement of the $\beta$-subunit. This was attributed mainly to the downward shift of AT domains by $10 \AA$, an outward rotation of the MPT by $9 \AA$ along with smaller shifts in DH (5 $\AA$ ) and ER domains ( $3 \AA$ ). It was argued that the cryo-EM structure represented the catalytically active conformation as the ACP domains were present at different active sites unlike in the crystal structures where they are always located at the KS domain. Stabilisation of an inactive conformation in crystals due to crystal contacts formed by the MPT domain was also hypothesised. However, a 4.9 A cryo-EM Chaetomium thermophilum 
FAS structure (Kastritis et al., 2017) contradicted the claims put forth by Gipson et al., (2010) by showing that the ACP can be stalled at a domain (ER) other than KS in the FAS conformation similar to the crystal structures.

It is thus unclear which conformation the FAS adopts during fatty acid synthesis - the one from the crystal structures or the one from S.cerevisiae cryo-EM structure. Along with this, whether the conformation of the $\beta$-subunit of the FAS affects the ACP domain's ability to interact with the different catalytic is also an open question? The answers to these questions are important for understanding how the elaborate structure of the fungal FAS allows it to perform synthesis of fatty acids.

\subsection{Tools for improving sample preparations}

\subsubsection{Chromatography-free purification}

Methods used for purifying large macromolecular complexes usually rely on tagging a subunit of the complex for affinity-based purification, along with other chromatography steps based on anion exchange and size exclusion. These methods are successful with small proteins $(<100 \mathrm{kDa})$ but can have detrimental effects on larger protein complexes (Liu et al., 2001). Multi-protein complexes tend to dissociate under high ionic strength. Along with this, shear forces due to interactions with the resin used in chromatography can cause the loss of bound subunits (Tsumoto et al., 2007). A milder purification procedure used to purify human 20S/26S proteasomes offers an alternative way of purifying large complexes (Haselbach et al., 2017; Schrader et al., 2016). This strategy involves precipitating proteins using polyethylene glycol (PEG) along with sucrose gradients while maintaining low ionic strength. $20 \mathrm{~S}$ proteasomes purified using this method were shown to be 10 times more active than those purified using conventional chromatography based purifications (Schrader et al., 2016). The crystals from such a preparations also diffracted up to $1.8 \AA$ which was a significant improvement compared to the previously reported $2.6 \AA$ structure of the human 20 S proteasome (Harshbarger et al., 2015). Such low salt, chromatographyfree methods offer an alternative and need to be tested on different protein complexes to check if they indeed are feasible and aid in achieving better resolutions. 


\subsubsection{GraFix}

Gradient Fixation (GraFix) of macromolecular complexes can be used for mild fixation of macromolecular complexes (Kastner et al., 2008). In this method, purified protein complexes are loaded onto a linear density gradient containing a fixative. During ultracentrifugation, the protein complexes sediment while undergoing mild crosslinking due to the gradual increase in crosslinker concentration. This method has been shown to stabilize protein complexes and prevent dissociation of subunits during cryo-EM sample preparation. GraFix has previously been used in the high resolution studies of $26 \mathrm{~S}$ proteasomes, spliceosomes and the MHC-I complex (Bertram et al., 2017; Blees et al., 2017; Haselbach et al., 2017). To date, this method has only been applied to compositionally stabilize protein complexes. In this thesis, I investigated the applicability of GraFix for conformational stability of the FAS and its ACP domains.

\subsubsection{Nanobodies}

Nanobodies are antigen-binding domains derived from variable domains (VHH) of camelid (or shark) heavy chain only antibodies. They are 13-15 kDa in size and contain three variable loops regions (CDR 1,2,3), which determine their binding affinity and specificity (Muyldermans, 2013). Nanobodies against a particular antigen can be generated by two methods (Liu et al., 2018): (1) Immunizing camelids with the antigen. Lymphocytes from the animals are then isolated and sequences of the VHH domains are amplified from cDNA libraries generated from these cells. The sequences for the VHH domains are ligated into vectors for phage display. The $\mathrm{VHH}$ domains expressed on the surface of bacteriophages are then selected for using immobilized antigens to identify high affinity binders. (2) In vitro libraries of nanobodies with randomized CDR sequences can also be directly used for phage display based screening against antigens. In comparison to conventional antibodies or Fab fragments, nanobodies are easy to produce as they can be expressed in E. coli or yeast, they are stable and their small size allows them to have better accessibility to epitopes (Muyldermans, 2013). In structural studies, nanobodies have been used for purifying proteins, as chaperones to assist in crystallization of other proteins and 
also for stalling specific conformational state of macromolecular complexes (Dmitriev et al., 2016; Duhoo et al., 2017; Pleiner et al., 2015; Steyaert and Kobilka, 2011). During this thesis, anti-FAS nanobodies were evaluated as a potential tool for stabilizing FAS conformations.

\subsubsection{Substrates/Inhibitors}

The conformational landscape of proteins can be altered by binding of small molecules (Cesa et al., 2015). This has been usually visualized for proteins in the form of snapshots of conformational differences with or without the substrates or inhibitors. This is also true for the fungal FAS where NADPH binding leads to rearrangement in an adjacent loop segment (881-875) of the KR domain (Jenni et al., 2007). Structures of the FAS in the presence of the other substrates or inhibitors mimicking reaction intermediates have not been reported and offer the possibility to visualize the different function states of this complex. Different combination of the FAS substrates (acetyl-CoA, malonyl-CoA and NADPH) along with inhibitors should be tested for this purpose. Additionally, the impact of such molecules on their binding site as well as on the whole complex would add to our understanding of how FAS performs its functions.

\subsubsection{In-silico sorting and analysis of protein conformations}

Along with improvements in biochemical preparations of protein complexes, computational methods can also be used to study and sort the composition as well as conformational heterogeneity in the sample. At present, most EM software packages accomplish this in a supervised or reference free manner using maximum likelihood estimation based algorithms (Grigorieff, 2016; Punjani et al., 2017; Zivanov et al., 2018). These procedures can either be used for in silico purification of a protein conformation and/or to determine the multiple 3D states a protein complex might adopt. However, this only hold true when complexes have distinct and stable conformational states (low energy states). Conformations representing continuous motions (high energy states) of a complex are still very difficult to distinguish. Proteins with such a behavior usually result in lowresolution structures comprising the multiple possible states. Therefore, along with 
determining the low energy states of proteins, methods for identifying and understanding high energy states that proteins might adopt are required. Conformational energy landscapes representing all the conformations present in cryo-EM datasets can be used in such scenarios as they can provide a more comprehensive understanding of dynamics of any given protein complex.

\subsubsection{Conformational energy landscapes using 3D Principle Component Analysis (PCA)}

Conformational landscape analysis for protein complexes can be performed using exhaustive 3D classification combined with 3D principle component analysis (3D PCA) (Haselbach et al., 2017, 2018). In this method, the total set of particles are split into numerous small subsets using 3D classification procedures. The obtained 3D volumes represent the different conformations of the complex present in the data. 3D Principle Component Analysis (PCA) is then performed to obtain the major modes of motion among the different $3 \mathrm{D}$ volumes. The resultant eigenimages representing the variance in the data can used to describe each 3D volume using the following equation

$$
X_{i}=\bar{X}+\sum_{j=1}^{n} a_{i, j} e_{i}
$$

, where every volume $X_{i}$ is described as a linear combination of the average volume $\bar{X}$ and the eigenvectors $e_{i}$ multiplied with the linear factor $a_{i, j}$. The first $\left(e_{1}\right)$ and second $\left(e_{2}\right)$ eigenvectors, which depict motions of compaction and rotation of the FAS dome, respectively, were selected for calculating conformational landscapes. The particle number belonging to each class can be used to calculate their free energies as multiples of the Boltzmann factor $\left(k_{B} T\right)$ :

$$
\Delta \Delta G=k_{B} T \ln \left(\frac{p_{i}}{p_{0}}\right)
$$

, where $T$ is the absolute temperature, $k_{B}$ the Boltzmann constant, $p_{i}$ is the number of particles in state $i$ and $p_{0}$ is the number of particles in the most populated state. The linear factors of $e_{1}$ and $e_{2}$ calculated as per equation (1) contributing to the input 3D volumes represent the $\mathrm{x}$ and $\mathrm{y}$ coordinates of the landscapes whereas the $\mathrm{z}$ axis represents the free energies of each class. 
3D PCA helps in identifying the major modes of motion present in a complex. This information can be used for 3D sorting while focusing only on the dynamic areas during image processing. Energy landscapes can also be used to study the global effect of small molecules or binding proteins on the conformation of a macromolecular complex.

\subsection{Aims and Scopes - Part I}

Structural characterization of fungal type I fatty acid synthases has been underway since early 1970's with the first high resolution structures determined in 2007. Structural data of these complexes to date comprise of one conformation solved at high resolution using Xray crystallography along with conflicting low-resolution structures using cryo-EM. Considering this, my aim was to gain insight into conformational changes of fungal type I fatty acid synthase during its catalytic cycle. In particular, the focus was on visualizing the tethered ACP bound to different enzymatic domains and to determine whether this correlates with the FAS dome conformation. For this purpose, different approaches such as mild fixation, substrate binding, nanobody-based stabilization and computational sorting were tested. During the course of this thesis, using the improved sample preparation conditions, I resolved two distinct conformations of the yeast FAS at sub-3 $\AA$ resolution. These are the highest resolution structures for this complex to date and provide new insights into the relationship between the structure and function of the FAS. Along with this, a novel FAS binding protein was co-purified using the newly established chromatography-free purification procedure. This protein was found to be involved in the regulation of FAS activity and was hence termed the $\gamma$-subunit. To understand the importance of this protein, a review of FAS regulation is therefore introduced below.

\subsection{Regulation of FAS in yeast}

Fatty acid synthesis is an energy demanding process. Energy rich metabolites produced during glycolysis and citric acid cycle such as acetyl-CoA, ATP, NADPH need to be shuttled into fatty acid synthesis based on nutrient availability and cellular requirements. For every palmitic acid (C16) synthesised, the cell uses the energetic equivalent of at least 122 ATP molecules (Wang et al., 2011). Therefore, if FAS activity is left unregulated, cellular survival 
is under threat due to possible overconsumption of resources by this machinery (Shpilka et al., 2015). The mechanisms that yeast cells employ for regulating FAS abundance and activity are described below and are similar to those found in higher eukaryotes.

\subsubsection{Transcriptional Regulation}

The two genes encoding the $\alpha$ - (FAS2) and $\beta$-subunit (FAS1) of yeast FAS are present on different chromosomes and their transcription needs to be co-ordinated for a balanced expression. Interestingly, other fungi don't have this problem as their FAS genes are usually arranged around the same promotor but oritented in opposite directions. The constitutive transcription of yeast FAS genes is mediated by general transcription factors - Rap1, Abf1, Reb1 for FAS1 and only by Reb1 for FAS2 (Schweizer and Hofmann, 2004). These transcription factors contribute towards $50 \%$ of the total expression levels of FAS genes. The rest is modulated by Inositol/Choline concentrations in the cell. Decrease in Inositol/Choline upregulates the levels of Ino2 and Ino4, which in turn activate FAS gene expression by binding and recruiting RNA polymerase to ICRE (Inositol/Choline Responsive Elements) elements in the upstream region of the FAS genes. On the other hand, increase in Inositol/Choline levels negatively regulates activation by Ino2/Ino4 through Opi1 and through histone deacetylation by Sin3 (Schweizer and Hofmann, 2004). Ino2/Ino4 and Opi1 also regulate ACC (synthesises malonyl-CoA) expression in a similar manner (Hasslacher et al., 1993).

\subsubsection{Translational regulation}

Translation of FAS2 is dependent on the levels of the $\beta$-subunit. It was first described in 2001 that the initial 66 nucleotides of the FAS2 gene contain a regulatory site, which depends on the $\beta$-subunit for translational activation (Wenz, 2001). The mechanism for this observation was provided using ribosome profiling by Shiber et al., (2018). Their data suggested that the ribosomes slow down/pause after translating the initial $\sim 94$ amino acids of the $\alpha$-subunit. These exposed amino acids are actually a part of and essential for the activity of MPT domain in the C-terminus of $\beta$-subunit. The MPT domain of the translated 
$\beta$-subunit interacts with the exposed amino acids of the $\alpha$-subunit resulting in activating translation and also promotes co-translational folding. This mechanism allows cells to have a stoichiometric expression of both the subunits.

\subsubsection{Post-translational modification}

The first committed step of fatty acid biosynthesis where acetyl-CoA is carboxylated by acetyl-CoA carboxylase 1 (ACC1) to form malonyl-CoA is regulated through posttranslational phosphorylation. In conditions of high cellular ATP concentrations, yeast ACC1 is in its active non-phosphorylated form. However, when ATP concentrations decrease, the yeast ACC1 is inactivated through phosphorylation by AMP-activated protein kinase called SNF1. This leads to a decrease in malonyl-CoA concentrations resulting in lower FAS activity (Hedbacker and Carlson, 2008; Wei et al., 2016). There are no known direct modifications of the yeast FAS that can affect its activity.

\subsubsection{Post-translational degradation}

Even if either of the two FAS subunits are over-expressed with respect to each other, selective degradation of un-assembled FAS subunits allows the cell to balance the individual subunit concentrations. Orphan $\alpha$-subunits are degraded in the cytosol by 26S/30S proteasomes. For this, $\alpha$-subunits are ubiquitinated in the presence of Hsp70 chaperone, Ssa1 by E2 ubiquitin-conjugating enzymes Ubc2/Ubc4 and E3 ubiquitin ligase Ubr1 (Egner et al., 1993; Scazzari et al., 2015). The ubiquitinated proteins are then acted upon by Cdc48 AAA-Atpase to disassemble the proteins for degradation by proteasomes. On the other hand, assembled FAS complexes or unassembled $\beta$-subunits are transported to vacuoles in a Atg8 dependent manner for their degradtion by vacuolar proteases like yscA and yscB (Egner et al., 1993; Shpilka et al., 2015). The degradation of FAS is important especially during nitrogen stress where cellular survival depends upon reduced FAS activity (Shpilka et al., 2015). 


\subsubsection{Metabolite based regulation}

The final known layer of FAS regulation involves short-term activation or inhibition of enzyme activity by metabolites. Even though direct evidence for yeast Acc1 is missing, it is known that this enzyme is allosterically activated by citrate but is inhibited by end products of fatty acid synthesis, palmitic- and steric-CoAs (Hasslacher et al., 1993; Hunkeler et al., 2016; Wakil et al., 1983). At high intracellular glucose levels, activation of Acc1 promotes increase in FAS activity due to the increase in malonyl-CoA concentrations. In turn, excess fatty acids produced regulate FAS by inhibting malonyl-CoA production by Acc1. Excess fatty acids also inhibit FAS directly and thus are important for feedback regulation based on cellular needs. The mechanism of inhibition of FAS by fatty acids is not well understood. In vitro studies have also demostrated that the ratio of acetyl-CoA/Malonyl-CoA can effect the chain length of fatty acids produced by the FAS (Sumper et al., 1969).

\subsection{Aims and Scopes - Part II}

During my PhD work, I established a chromatography-free purification of the S. cerevisiae FAS. Interestingly, a $17 \mathrm{kDa}$ protein was found to co-purify with the FAS. Since a specific fungal FAS binding protein has not been reported to date, I aimed to characterize the role of this $17 \mathrm{kDa}$ protein with respect to the FAS. To this end, I performed biochemical and structural analysis of the FAS in the presence and absence of this $17 \mathrm{kDa}$ protein. This protein was found to bind inside the FAS and interact with three catalytic active sites. Additionally, it lowers the affinity of the FAS for all its substrates and affects the conformational landscape of the FAS. Due to its substantial effect on the FAS activity and structure, this protein was termed as the " $\gamma$-subunit" of the FAS. 


\section{Materials and Methods}

\subsection{Materials}

\subsubsection{Special Equipment}

\begin{tabular}{l|l}
\hline Equipment & Manufacturer \\
\hline ActiLoops & Molecular dimensions \\
Amicon ultra 15 centrifugal concentrator & Millipore \\
Balances & Sartorius \\
Centrifuge & LYNX 6000 Thermo Scientific \\
Copper EM grids & Plano \\
Cryschem M Plate (24-well) & Hampton Research \\
Crystal Clear Sealing Tape & Jena Bioscience \\
Crystalgen pregreased Plate (24-well), & Jena Bioscience \\
Dialyzer mini D-tube & Millipore \\
Electro Supplier Power Pac 3000 & BioRad \\
EmulsiFlex-C3 & Avestin Inc. \\
Fiberlite rotor F14/ F21/ F35L & Thermo Scientific \\
Fluoromax-4 fluorescence & Horiba Scientific \\
spectrophotometer & \\
Gradient Master & ip Biocomp \\
Incubator Eco-Line E100 & RUMED \\
Incubator Multitron Pro Shaker & Infors HT \\
LithoLoops & Molecular dimensions \\
MicroLoops & MiTeGen \\
Orbital Shaker RS-S20 & Phoenix Instrument \\
Photometer Lambda Bio X & Perkin Elmer \\
Pipetting Robot Microlab Star LET & Hamilton \\
Quantifoil grid R3.5/1 Cu 200 mesh & Quantifoil MicroTools GmbH \\
Rotor TH660 & Thermo Scientific \\
Rotor S140AT & Thermo Scientific \\
Rotor SW40 & Beckmann Coulter \\
Siliconized Glass Cover Slides & Hampton Research \\
Stereo microscopes M125 & Leica \\
TEM CM200 FEG & Philips \\
TEM Titan Krios & Thermo Scientific \\
Ultra-Centrifugal Mill ZM 200 & Retsch® \\
Ultracentrifuge WX Ultra 80/ 90 & Thermo Scientific \\
V-750 UV-Visible spectrophotometer & Jasco instruments \\
Vitrobot & Thermo Scientific \\
Zebra Micro Desalt Spin Column & Thermo Scientific \\
\hline
\end{tabular}




\subsubsection{Chemicals}

\begin{tabular}{|c|c|}
\hline Chemicals & Supplier \\
\hline Acetic acid & Merck \\
\hline Acetonitrile & Merck \\
\hline Acetyl-CoA & Sigma \\
\hline Amido Black 10B & SERVA Electrophoresis \\
\hline Ammonium sulfate & Merck \\
\hline Benzamidine Hydrochloride hydrate & Sigma-Aldrich \\
\hline$\beta$-mercaptoethanol ( $\beta$-ME) & Sigma-Aldrich \\
\hline Biotin-dPEGTM(24)-NHS & Iris biotech \\
\hline Bis(sulfosuccinimidyl)suberate (BS3) & Thermo Scientific \\
\hline $\begin{array}{l}\text { Bis(2-hydroxyethyl)aminotris(hydroxymethyl)methane } \\
\text { (BisTris) }\end{array}$ & Sigma-Aldrich \\
\hline Boric acid & Merck \\
\hline Bradford Assay reagent & BioRad \\
\hline Coomassie Brilliant Blue R-250 & BioRad \\
\hline Dimethyl sulfoxide (DMSO) & AppliChem \\
\hline Dithiothreitol (DTT) & Roth \\
\hline DNase & Jeno Bioscience \\
\hline Ethanol & Merck \\
\hline Ethylenediaminetetraacetic acid (EDTA) & Merck \\
\hline Glucose & Merck \\
\hline Glutaraldehyde & Science Services \\
\hline Glycerol & Merck \\
\hline Hydrochloric acid $(\mathrm{HCl})$ & Merck \\
\hline Iodoacetamide & Sigma-Aldrich \\
\hline Isopropyl ß-D-1-thiogalactopyranoside (IPTG) & Sigma-Aldrich \\
\hline Isopropanol & Merck \\
\hline Lauryl maltose neopentyl glycol (LMNG) & Anatrace \\
\hline Lyzozyme & Thermo Scientific \\
\hline Magnesium chloride $(\mathrm{MgCl} 2)$ & Merck \\
\hline Magnesium acetate $(\mathrm{Mg}(\mathrm{CH} 3 \mathrm{COO}) 2)$ & Merck \\
\hline Malonyl-CoA & Sigma-Aldrich \\
\hline NHS-rhodamine & Thermo Scientific \\
\hline Nicotinamide adenine dinucleotide (NADH) & Roth \\
\hline Nicotinamide adenine dinucleotide phosphate (NADPH) & Roth \\
\hline 2-(N-morpholino)ethanesulfonic acid (MES) & Sigma-Aldrich \\
\hline Octyl glucose neopentyl glycol (OGNG) & Anatrace \\
\hline Polyethylene glycol (PEG) 400 & Sigma-Aldrich \\
\hline PEG 3350 & Sigma-Aldrich \\
\hline Peptone/tryptone & Roth \\
\hline Phenylmethane sulfonyl fluoride (PMSF) & Sigma-Aldrich \\
\hline Potassium acetate & Merck \\
\hline Potassium chloride $(\mathrm{KCl})$ & Merck \\
\hline
\end{tabular}


Potassium hydroxide $(\mathrm{KOH})$

All Blue Protein Standard

Sucrose

Sodium Aspartate

Sodium chloride $(\mathrm{NaCl})$

Sodium malonate

SDS-PAGE running buffer

Terrific broth

Tri-fluoro acetic acid (TFA)

Tris(hydroxymethyl)aminomethane (Tris)

Yeast extract

Uranyl formate
Merck

BioRad

Merck

Sigma-Aldrich

Merck

Sigma-Aldrich

Roth

Melford biolabratory

Merck

VWR chemicals

Roth

Custom made

\subsubsection{Buffer composition}

\begin{tabular}{|c|c|}
\hline \multirow{3}{*}{ Purification Buffer: } & 0.05 M BisTris pH 6.5 \\
\hline & $0.05 \mathrm{M}$ potassium acetate \\
\hline & $0.01 \mathrm{M}$ magnesium acetate \\
\hline \multirow{4}{*}{ Crystallization Buffer A: } & $0.1 \mathrm{M}$ HEPES pH 7.0 \\
\hline & $0.3 \mathrm{M}$ sodium-malonate \\
\hline & $\mathrm{pH} 7.0$ \\
\hline & $11.5-13 \%(w / v)$ PEG3350 \\
\hline \multirow{4}{*}{ Crystallization Buffer B: } & 0.1 M MES pH 6.2 \\
\hline & 0.15-0.3 M ammonium \\
\hline & sulphate \\
\hline & 7-8\% (w/v) PEG 8000 \\
\hline \multirow{4}{*}{ Stabilization buffer A: } & 0.1 M HEPES pH 7.0 \\
\hline & $0.3 \mathrm{M}$ sodium malonate \\
\hline & $\mathrm{pH} 7.0$ \\
\hline & $15 \%(w / v)$ PEG3350 \\
\hline \multirow{3}{*}{ Stabilization buffer B: } & 0.1 M MES pH 6.2 \\
\hline & $0.3 \mathrm{M}$ ammonium sulphate \\
\hline & 12\% (w/v) PEG 8000 \\
\hline \multirow{3}{*}{ Kinetic assay buffer: } & 0.05 M BisTris pH 6.5 \\
\hline & $0.05 \mathrm{M}$ potassium acetate \\
\hline & $0.5 \mathrm{mM}$ magnesium acetate \\
\hline
\end{tabular}




\subsubsection{Software}

\begin{tabular}{|c|c|c|}
\hline Software & Source & Reference \\
\hline $\begin{array}{l}\text { Adobe Creative } \\
\text { Suite } 5\end{array}$ & http://www.adobe.com/de/creativecloud.html & \\
\hline $\mathrm{CCP} 4$ & http://www.ccp4.ac.uk & $\begin{array}{l}\text { (Winn et al., } \\
\text { 2011) }\end{array}$ \\
\hline COW suite & $\begin{array}{l}\text { Department of Structural Dynamics, } \\
\text { Max-Planck-Institute for Biophysical Chemistry }\end{array}$ & \\
\hline COOT & $\begin{array}{l}\text { https://www2.mrclmb.cam.ac.uk/ } \\
\text { personal/pemsley/coot }\end{array}$ & $\begin{array}{l}\text { (Emsley and } \\
\text { Cowtan, 2004) }\end{array}$ \\
\hline Gautomatch & http://www.mrc-lmb.cam.ac.uk/kzhang & \\
\hline Gctf & http://www.mrclmb.cam.ac.uk/kzhang & (Zhang, 2016) \\
\hline JLigand & http://www.ysbl.york.ac.uk/mxstat/JLigand & $\begin{array}{l}\text { (Lebedev et al., } \\
\text { 2012) }\end{array}$ \\
\hline OriginPro 9.1 & http://www.originlab.de/Origin & \\
\hline Phenix & https://www.phenix-online.org & $\begin{array}{l}\text { (Adams et al., } \\
\text { 2010) }\end{array}$ \\
\hline Python 2.7 & https://www.python.org & \\
\hline PyMOL & https://www.pymol.org & $\begin{array}{l}\text { (Schrödinger, } \\
\text { 2015) }\end{array}$ \\
\hline Refmac5 & $\frac{\text { https://www2.mrc-lmb.cam.ac.uk/groups/ }}{\text { murshudov/content/refmac/refmac.html }}$ & $\begin{array}{l}\text { (Murshudov et } \\
\text { al., 2011) }\end{array}$ \\
\hline RELION 2.0/3.0 & http://www2.mrc-lmb.cam.ac.uk/relion & $\begin{array}{l}\text { (Scheres, 2012; } \\
\text { Zivanov et al., } \\
\text { 2018) }\end{array}$ \\
\hline Robetta & http://robetta.bakerlab.org/ & (Kim et al., 2004) \\
\hline Staraniso Server & $\begin{array}{l}\text { http://staraniso.globalphasing.org/ } \\
\text { cgibin/staraniso.cgi }\end{array}$ & $\begin{array}{l}\text { (Tickle, I. } \\
\text { Flensburg, C. } \\
\text { Keller, P. } \\
\text { Paciorek, W. } \\
\text { Sharff, A. } \\
\text { Vonrhein, C. } \\
\text { Bricogne, 2018) }\end{array}$ \\
\hline UCSF Chimera & http://www.cgl.ucsf.edu/chimera & $\begin{array}{l}\text { (Pettersen et al., } \\
\text { 2004) }\end{array}$ \\
\hline UCSF Chimera X & https://www.cgl.ucsf.edu/chimerax/ & (Goddard et al., \\
\hline $\begin{array}{l}\text { XDS Program } \\
\text { Package }\end{array}$ & http://xds.mpimf-heidelberg.mpg.de & (Kabsch, 2010) \\
\hline
\end{tabular}




\subsection{Methods}

\subsubsection{Cell culture}

\subsubsection{Chaetomium thermophilum culture}

Chaetomium thermophilum La Touche var. thermophilum was grown in YPD medium in an Infors 150 liter fermenter and harvested in late log phase at an OD600 of 9-10. Subsequently, cells were washed with cold $\mathrm{ddH}_{2} \mathrm{O}$, dried and then flash frozen in liquid nitrogen.

\subsubsection{Saccharomyces cerevisiae culture}

All yeast manipulations were performed as per standard protocols (Gietz, 2014). Saccharomyces cerevisiae strains BJ2168 (MATa prc1-407 prb1-1122 pep4-3 leu2 trp1 ura3-52 gal2) and Tma17 B BJ2168 (MATa prc1-407 prb1-1122 pep4-3 leu2 trp1 ura3-52 gal2 Tma17::kanMX) were used in this study. Cells were grown in YPD medium in an Infors 250 litre fermenter and harvested in late log phase at an $\mathrm{OD}_{600}$ of 9-10. Subsequently, cells were washed with cold $\mathrm{ddH}_{2} \mathrm{O}$ and then resuspended in $2 \mathrm{X}$ cell resuspension buffer $(0.05 \mathrm{M}$ BisTris $\mathrm{pH}$ 6.5, 0.05 M potassium chloride, 0.01 M magnesium chloride) containing 20\% $(\mathrm{w} / \mathrm{v})$ sucrose such that $2 \mathrm{ml}$ of buffer was added per gram of cells. Cells were then flash frozen as beads in liquid nitrogen and stored at $-80^{\circ} \mathrm{C}$ until further use.

\subsubsection{Protein Purification}

\subsubsection{Purification of Chaetomium thermophilum FAS (CtFAS)}

The purification strategy was adapted from protocols developed earlier for the purification of human 20S and 26S proteasomes (Haselbach et al., 2017; Schrader et al., 2016). $180 \mathrm{~g}$ of frozen cell pellets were ground in liquid nitrogen to a fine powder using a Retsch ZM200 mill. The ground powder was thawed in a water bath at $37^{\circ} \mathrm{C}$, supplemented with $420 \mathrm{~mL}$ of purification buffer containing $20 \mathrm{mM}$ DTT followed by the addition of benzamidine chloride to $10 \mathrm{mM}$ and PMSF to $5 \mathrm{mM}$ (from a $100 \mathrm{mM}$ stock solution in propanol). The extract was incubated at $25{ }^{\circ} \mathrm{C}$ on a magnetic stirrer for 30 minutes followed by 
centrifugation at $30,000 \times \mathrm{g}$ for 30 minutes at $4{ }^{\circ} \mathrm{C}$. After centrifugation, the supernatant was filtered through 3 layers each of cheese cloth and miracloth to obtain a S30 cell extract. This was then centrifuged at $100,000 \times \mathrm{g}$ for 1 hour at $4{ }^{\circ} \mathrm{C}$. The supernatant was again filtered through 3 layers each of cheese cloth and miracloth. The obtained clarified S100 extract was subjected to differential precipitation with $\underline{P}$ olyEthylenelycol400 (PEG; number signifies the mean molecular weight of the PEG polymer). PEG400 was added to a concentration of $15 \%(\mathrm{v} / \mathrm{v})$ to the $\mathrm{S} 100$ extract while stirring at $18{ }^{\circ} \mathrm{C}$ and incubated for 30 minutes. Precipitated proteins were removed by centrifugation at $30,000 \mathrm{xg}$ for 30 minutes at $4{ }^{\circ} \mathrm{C}$. The precipitate of this step was recovered by centrifugation at 30,000 $\mathrm{g}$ for 30 minutes at $4{ }^{\circ} \mathrm{C}$ and resuspended in purification buffer containing $2 \%(\mathrm{w} / \mathrm{v})$ sucrose, $10 \mathrm{mM}$ DTT and $0.01 \%(w / v)$ Lauryl Maltose Neopentyl Glycol (LMNG) in an orbital shaker at $18{ }^{\circ} \mathrm{C}$. The resuspended material was loaded on $10-45 \%(\mathrm{w} / \mathrm{v})$ linear sucrose gradients in purification buffer containing $10 \mathrm{mM}$ DTT, which were centrifuged at $100,000 \mathrm{x} \mathrm{g}$ for $16 \mathrm{~h}$ at $4{ }^{\circ} \mathrm{C}$. Gradients were harvested in $1 \mathrm{ml}$ fractions. SDS-PAGE was utilized to identify fractions containing FAS. Selected fractions were pooled, treated with $10 \mathrm{mM}$ NADH for 30 minutes at $18{ }^{\circ} \mathrm{C}$ and then precipitated by addition of $8 \%(v / v)$ PEG3350. After centrifugation (30,000 $\mathrm{x} g$, 30 minutes), the supernatant was then precipitated by raising the concentration of PEG3350 to 10\%. The precipitate of this step, which contained the FAS, was resuspended in purification buffer containing 2\% (w/v) sucrose, $10 \mathrm{mM}$ DTT and 0.01\% (w/v) LMNG. The resuspended material was loaded on linear $10-45 \%(\mathrm{w} / \mathrm{v})$ sucrose gradients in purification buffer containing $10 \mathrm{mM} \mathrm{DTT}$, and centrifuged at 79,000 x g for $16 \mathrm{~h}$ at $4{ }^{\circ} \mathrm{C}$. Fractions containing FAS were identified by SDS-PAGE, pooled and precipitated by the addition of $40 \%(\mathrm{v} / \mathrm{v})$ PEG400 and resuspended in purification buffer containing 10\% (w/v) sucrose, 10 $\mathrm{mM}$ DTT and $0.01 \%(\mathrm{w} / \mathrm{v})$ LMNG. Protein concentrations were determined by Bradford assay (BioRad, Munich, Germany) using a BSA standard and protein was stored at a concentration of $12 \mathrm{mg} / \mathrm{ml}$.

\subsubsection{Purification of $S$. cerevisiae FAS (ScFAS)}

$700 \mathrm{~g}$ of frozen cell beads (corresponding to $233 \mathrm{~g}$ wet cell weight of yeast) were ground in liquid nitrogen to a fine powder using a Retsch ZM200 mill. The ground powder was 
thawed in a water bath at $37{ }^{\circ} \mathrm{C}$, supplemented with purification buffer to $0.33 \mathrm{x}$ concentration from a 10x stock, followed by the addition of sucrose powder to $20 \%(\mathrm{w} / \mathrm{v})$, benzamidine chloride to $10 \mathrm{mM}$ and PMSF to $1 \mathrm{mM}$ (from a $100 \mathrm{mM}$ stock solution in propanol). The extract was incubated at $25^{\circ} \mathrm{C}$ on a magnetic stirrer for 30 minutes followed by centrifugation at $30,000 \times \mathrm{g}$ for 30 minutes at $4{ }^{\circ} \mathrm{C}$. After centrifugation, the supernatant was filtered through 3 layers each of cheese cloth and miracloth to obtain a S30 yeast cell extract. To this, Octyl Glucose Neopentyl Glycol (OGNG) (from a 10\% (w/v) stock) was added to a final concentration of $0.2 \%(\mathrm{v} / \mathrm{v})$ and the extract was incubated at $30{ }^{\circ} \mathrm{C}$ for 30 minutes followed by centrifugation at $100,000 \times \mathrm{g}$ for 1 hour at $4{ }^{\circ} \mathrm{C}$. The supernatant was again filtered through 3 layers each of cheese cloth and miracloth. The thus clarified S100 extract was subjected to differential precipitation with PolyEthyleneGlycol400 (PEG; number signifies the mean molecular weight of the PEG polymer). PEG400 was added to a concentration of $20 \%(\mathrm{v} / \mathrm{v})$ to the yeast $\mathrm{S} 100$ extract while stirring at $18{ }^{\circ} \mathrm{C}$ and incubated for 30 minutes. Precipitated proteins were removed by centrifugation at $30,000 \times \mathrm{g}$ for 30 minutes at $4{ }^{\circ} \mathrm{C}$. The supernatant was then precipitated by raising the concentration of PEG400 to $30 \%(\mathrm{v} / \mathrm{v})$ as described above. The precipitate of this step, which contains the FAS, was recovered by centrifugation at $30,000 \times \mathrm{g}$ for 30 minutes at $4{ }^{\circ} \mathrm{C}$ and resuspended in purification buffer containing $2 \%(\mathrm{w} / \mathrm{v})$ sucrose, $10 \mathrm{mM}$ DTT and $0.01 \%(\mathrm{w} / \mathrm{v})$ Lauryl Maltose Neopentyl Glycol (LMNG) in an orbital shaker at $18^{\circ} \mathrm{C}$. The resuspended material was loaded on $10-45 \%(\mathrm{w} / \mathrm{v})$ linear sucrose gradients in purification buffer containing 10 mM DTT, which were centrifuged at $100,000 \times \mathrm{g}$ for $16 \mathrm{~h}$ at $4{ }^{\circ} \mathrm{C}$. Gradients were harvested in $1 \mathrm{ml}$ fractions. SDS-PAGE was utilized to identify fractions containing FAS. Selected fractions were pooled and precipitated by the addition of $40 \%$ (v/v) PEG400. After centrifugation $(30,000 \times \mathrm{g}$, 30 minutes), the supernatant was removed and the precipitate was resuspended in purification buffer containing $2 \%(\mathrm{w} / \mathrm{v})$ sucrose, $10 \mathrm{mMDTT}$ and $0.01 \%$ $(\mathrm{w} / \mathrm{v})$ LMNG. The resuspended material was loaded on linear $10-45 \%(\mathrm{w} / \mathrm{v})$ sucrose gradients in purification buffer containing $10 \mathrm{mM} \mathrm{DTT}$, and centrifuged at 79,000 $\mathrm{g}$ for 16 $\mathrm{h}$ at $4{ }^{\circ} \mathrm{C}$. Fractions containing FAS were identified by SDS-PAGE, pooled and cycled in the presence of $50 \mu \mathrm{M}$ Malonyl-CoA and $100 \mu \mathrm{M}$ of NADPH for $30 \mathrm{~min}$ at $18^{\circ} \mathrm{C}$ (this step of substrate addition was performed for FAS preparations after the structural analysis of the endogenous FAS holoenzyme complex). The protein was precipitated and concentrated by 
the addition of $40 \%(\mathrm{v} / \mathrm{v})$ PEG400 and resuspended in purification buffer containing $2 \%$ $(\mathrm{w} / \mathrm{v})$ sucrose, $10 \mathrm{mM}$ DTT and 0.01\% (w/v) LMNG. Another round of linear 10-45\% (w/v) sucrose gradients in purification buffer containing $10 \mathrm{mM}$ DTT, centrifuged at 60,000 x $\mathrm{g}$ for $16 \mathrm{~h}$ at $4{ }^{\circ} \mathrm{C}$ and subsequent re-precipitation of FAS fractions with $40 \%$ (v/v) PEG400 was required to yield a final purified protein preparation at $\sim 15 \mathrm{mg} / \mathrm{ml}$ in purification buffer containing 10\% sucrose (w/v), $10 \mathrm{mM}$ DTT and 0.01\% (w/v) LMNG. Protein concentrations were determined by Bradford assay (BioRad, Munich, Germany) using a BSA standard. This procedure reproducibly resulted in a yield of 15-20 mg purified yeast FAS.

\subsubsection{Expression and purification of the $\gamma$-subunit}

The $\gamma$-subunit was cloned as a synthetic gene into a pET151/D-TOPO ${ }^{\circledR}$ plasmid (Geneart, Regensburg) and expressed with an N-terminal His6-TEV-tag in BL21 Star (DE3) competent cells. The transformed cells were grown at $37^{\circ} \mathrm{C}$ with $180 \mathrm{rpm}$ shaking until an $\mathrm{OD}_{600}$ of 0.5 was reached, the temperature was then decreased to $18^{\circ} \mathrm{C}$ and expression of the $\gamma$-subunit was induced $1 \mathrm{~h}$ after reduction of the temperature by the addition of $0.5 \mathrm{mM}$ IPTG. $\gamma$ subunit was expressed for $16 \mathrm{~h}$ at $18{ }^{\circ} \mathrm{C}$ and $180 \mathrm{rpm}$. The cells were harvested by centrifugation $\left(5000 \times \mathrm{g}, 15\right.$ minutes, $\left.4{ }^{\circ} \mathrm{C}\right)$, washed with cold $\mathrm{ddH}_{2} \mathrm{O}$ and stored at $-80^{\circ} \mathrm{C}$.

For purification, 7 grams of cells were resuspended in $42 \mathrm{ml}$ of resuspension buffer $(20 \mathrm{mM}$ Tris- $\mathrm{HCl} \mathrm{pH}$ 8.0, $500 \mathrm{mM} \mathrm{NaCl}, 10 \mathrm{mM}$ imidazole, $0.2 \mathrm{mM}$ PMSF, $10 \mathrm{mM}$ benzamidine). After addition of $2 \mathrm{U} / \mathrm{ml}$ DNAse and $0.33 \mathrm{mg} / \mathrm{ml}$ lysozyme, the suspension was incubated for $30 \mathrm{~min}$ at $4{ }^{\circ} \mathrm{C}$. Cells were lysed by passing twice through an Avestin Emulsiflex C3 fluidizer (Avestin, Mannheim, Germany) at 15,000 PSI. The obtained lysate was centrifuged (30 min, 50,000 $\mathrm{g}, 4^{\circ} \mathrm{C}$ ) and filtered through Minisart NML Plus cellulose-acetate syringe filters with a $0.45 \mu \mathrm{m}$ pore size (Sartorius). The cleared lysate was loaded onto a Ni-NTA gravity column (5 $\mathrm{ml}$ bed volume) pre-equilibrated with 20 column volumes (CV) of resuspension buffer. The column was washed with $20 \mathrm{CV}$ resuspension buffer, followed by $20 \mathrm{CV}$ of washing buffer $(20 \mathrm{mM}$ Tris- $\mathrm{HCl} \mathrm{pH}$ 8.0, $500 \mathrm{mM} \mathrm{NaCl}, 20 \mathrm{mM}$ imidazole, $0.2 \mathrm{mM}$ PMSF, $10 \mathrm{mM}$ benzamidine). For elution of the bound Tma17p, $5 \mathrm{CV}$ of elution buffer (20 $\mathrm{mM}$ Tris- $\mathrm{HCl} \mathrm{pH}$ 8.0, $500 \mathrm{mM} \mathrm{NaCl}, 500 \mathrm{mM}$ imidazole, $0.2 \mathrm{mM}$ PMSF, $10 \mathrm{mM}$ benzamidine) were applied and collected in $2 \mathrm{ml}$ fractions. The elution fractions were 
analyzed with SDS-PAGE, the Tma17p containing fractions pooled, and the protein concentration photometrically determined $\left(\mathrm{MW}=17.4 \mathrm{kDa}, \varepsilon_{280}=5960\right)$. To cleave the $\mathrm{N}$ terminal His6-TEV-tag, TEV-protease was added to the protein in an enzyme-to-protein ratio of 1:50 followed by dialysis overnight in 51 dialysis buffer ( $20 \mathrm{mM}$ Tris- $\mathrm{HCl} \mathrm{pH}$ 8.0, 5 $\mathrm{mM} \beta-\mathrm{ME}, 100 \mathrm{mM} \mathrm{NaCl}$ ) at $4^{\circ} \mathrm{C}$. The digested Tma17p was applied to a Ni-NTA column, which was pre-equlilibrated with $20 \mathrm{CV}$ dialysis buffer. The flow-through was collected in $2 \mathrm{ml}$ fractions and single fractions were analyzed by SDS-PAGE. Fractions containing pure Tma17p were pooled and concentrated to a final concentration of $20 \mathrm{mg} / \mathrm{ml}$ using a $10 \mathrm{kDa}$ MWCO Amicon Ultra 15 centrifugal concentrator (Millipore). The final protein was aliquoted, frozen in liquid nitrogen and stored at $-80^{\circ} \mathrm{C}$. For further kinetic and structural investigations, purified Tma17p was thawed and dialysed into the kinetic assay buffer at 4 ${ }^{\circ} \mathrm{C}$ using 6-8 kDa MWCO Dialyzer Mini D-Tubes (Millipore).

\subsubsection{Nanobody expression and purification}

Nanobody cloned into cytoplasmic expression and characterization vector pTP898 were transformed into E. coli BL21 DE3 cells and cultured in TB medium containing kanamycin. The transformed cells were grown at $37^{\circ} \mathrm{C}$ with $180 \mathrm{rpm}$ shaking until an OD 600 of 0.6 was reached, the temperature was then decreased to $25^{\circ} \mathrm{C}$. The expression was induced $1 \mathrm{~h}$ after reduction of the temperature by the addition of $0.2 \mathrm{mM}$ IPTG. The nanobody was expressed for $5 \mathrm{~h}$ at $25{ }^{\circ} \mathrm{C}$ and $180 \mathrm{rpm}$. The cells were harvested by centrifugation $(5000 \times \mathrm{g}, 15$ minutes, $4{ }^{\circ} \mathrm{C}$ ), washed with cold $\mathrm{ddH}_{2} \mathrm{O}$ and stored at $-80^{\circ} \mathrm{C}$.

For purification, 4 grams of cells were resuspended in $20 \mathrm{ml}$ of resuspension buffer $(50 \mathrm{mM}$ Tris- $\mathrm{HCl}$ pH 7.5, 300 mM NaCl, 20 mM imidazole, 1 mM PMSF, 10 mM benzamidine). After addition of $2 \mathrm{U} / \mathrm{ml}$ DNAse and $0.33 \mathrm{mg} / \mathrm{ml}$ lysozyme, the suspension was incubated for 30 min at $4{ }^{\circ} \mathrm{C}$. Cells were lysed by sonication for 2 minutes at $50 \%$ amplitude and a pulse of 2 sec on/off. The resulting lysate was centrifuged $\left(30 \mathrm{~min}, 50,000 \mathrm{~g}, 4^{\circ} \mathrm{C}\right)$ and filtered through Minisart NML Plus cellulose-acetate syringe filters with a $0.45 \mu \mathrm{m}$ pore size (Sartorius). The cleared lysate was loaded onto a Ni-NTA gravity column (2 $\mathrm{ml}$ bed volume) preequilibrated with 20 column volumes $(\mathrm{CV})$ of resuspension buffer. The column was washed with $30 \mathrm{CV}$ resuspension buffer. The beads were then incubated with $5 \mathrm{ml}$ resuspension 
buffer containing $1 \mu \mathrm{M}$ SUMO protease for $2 \mathrm{~h}$. The column was then washed with $30 \mathrm{CV}$ resuspension buffer to remove the cleaved tags. For elution of the bound nanobody, $8 \mathrm{CV}$ of elution buffer $(50 \mathrm{mM}$ Tris- $\mathrm{HCl}$ pH 7.5, $300 \mathrm{mM} \mathrm{NaCl}, 500 \mathrm{mM}$ imidazole, $0.1 \mathrm{mM}$ benzamidine) were applied and collected in $2 \mathrm{ml}$ fractions. The elution fractions were analyzed with SDS-PAGE, the nanobody containing fractions pooled, and the protein concentration photometrically determined. The protein was dialyzed overnight in $50 \mathrm{mM}$ Bis-Tris acetate $\mathrm{pH}$ 6.5, $50 \mathrm{mM} \mathrm{KOAc,} 5 \mathrm{mM} \mathrm{Mg}(\mathrm{OAc}) 2,5 \mathrm{mM}$ DTT and 8.5\% sucrose. After concentrating using a $4 \mathrm{kDa}$ MWCO Amicon Ultra 15 centrifugal concentrator (Millipore), the protein was aliquoted, frozen in liquid nitrogen and stored at $-80{ }^{\circ} \mathrm{C}$.

\subsubsection{Biochemical Assays}

\subsubsection{Binding affinity of the $\gamma$-subunit to FAS}

To determine the binding affinity of the $\gamma$-subunit to the FAS, $\gamma$-subunit was labelled with NHS-Rhodamine (Thermo Fisher Scientific) by incubating the protein with the dye in a 1:10 ratio for $1 \mathrm{hr}$ at $25^{\circ} \mathrm{C}$ in dark. The mixture was passed through a desalting column to remove excess unreacted dye. Different concentrations of NHS-Rhodamine labelled $\gamma$-subunit were titrated against 15.38 pmol FAS in assay buffer in a total reaction volume of $40 \mu 1$. The mix was incubated at $30{ }^{\circ} \mathrm{C}$ for 30 minutes. $10 \mu$ l of each reaction were then loaded onto a $1.5 \%$ $(\mathrm{w} / \mathrm{v})$ agarose gel (in $0.5 \mathrm{X}$ TBE, $2 \mathrm{mM} \mathrm{MgCl}$ ) and run at $75 \mathrm{~mA}$ for 2 hours at $4{ }^{\circ} \mathrm{C}$. The fluorescence signal from the FAS- $\gamma$-subunit complex was imaged using an Amersham imager 600 (GE Healthcare) and quantified using ImageJ (Schindelin et al., 2012). The dissociation constant of the $\gamma$-subunit was calculated using the Hill equation.

\subsubsection{Salt concentration-dependent dissociation of the $\gamma$-subunit}

FAS holoenzyme $(0.52 \mathrm{mg})$ was loaded on linear $10-45 \%(\mathrm{w} / \mathrm{v})$ sucrose gradients in purification buffer containing 10mM DTT supplemented with either 0, 100, or $200 \mathrm{mM} \mathrm{KCl}$. The gradients were centrifuged at $120,000 \times \mathrm{g}$ for $16 \mathrm{~h}$ at $4{ }^{\circ} \mathrm{C}$ and were harvested in $200 \mu \mathrm{l}$ fractions. All fractions of the gradients at the three salt concentrations were then analyzed by SDS-PAGE. 


\subsubsection{Steady-state kinetics}

Kinetic measurements were carried out with a V-750 UV-Visible spectrophotometer (Jasco Instruments) by monitoring NADPH consumption during substrate turnover. The substrate concentration dependence for all three FAS substrates (i.e., NADPH, acetyl-CoA and malonyl-CoA) was analyzed. Commercial acetyl-CoA and malonyl-CoA were further

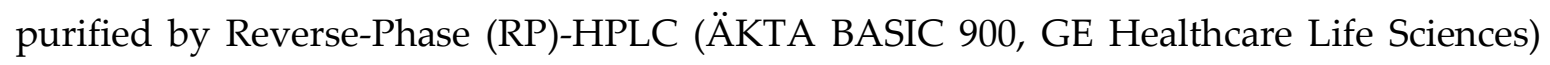
using a preparative column (MN Nucleodur ${ }^{\circledR} 100-5-C 18,250 \mathrm{~mm} \times 21 \mathrm{~mm}, 5$ m, flow rate: $10 \mathrm{ml} / \mathrm{min}$ ). UV-absorption was detected at $215 \mathrm{~nm}, 260 \mathrm{~nm}$ and $280 \mathrm{~nm}$, and purification was performed with a linear gradient of $\mathrm{A}$ (water $+0.1 \%(\mathrm{v} / \mathrm{v}) \mathrm{TFA})$ to $\mathrm{B}(79.9 \%$ acetonitrile $(\mathrm{v} / \mathrm{v})+20 \% \mathrm{ddH} 2 \mathrm{O}+0.1 \%(\mathrm{v} / \mathrm{v})$ TFA). The peak fractions of the corresponding substrates were collected, lyophilized and stored at $-80^{\circ} \mathrm{C}$ until use. The substrate stock concentrations were determined photometrically (NADPH: $\varepsilon_{340}=6220 \mathrm{M}^{-1} \mathrm{~cm}^{-1}, \varepsilon_{371}=2631.8 \pm 6.5 \mathrm{M}^{-1} \mathrm{~cm}^{-1}$; acetyl-CoA: $\varepsilon_{260}=15.400 \mathrm{M}^{-1} \mathrm{~cm}^{-1}$; malonyl-CoA: $\left.\varepsilon_{260}=15400 \mathrm{M}^{-1} \mathrm{~cm}^{-1}\right)$. Initial velocities of fatty acid synthesis at varying acetyl- and malonyl-CoA concentrations were determined by the measurement of the time-dependent change in absorption of NADPH at $\lambda=340 \mathrm{~nm}$. To study the concentration dependence for acetyl-CoA, malonyl-CoA $(120 \mu \mathrm{M})$, and NADPH $(360 \mu \mathrm{M})$ were kept constant, while acetyl-CoA concentrations were varied between 0-180 $\mu \mathrm{M}$. In the case of malonyl-CoA, acetyl-CoA $(180 \mu \mathrm{M})$ and NADPH $(360 \mu \mathrm{M})$ were kept constant, while the concentration of malonyl-CoA concentrations was varied from 0-180 $\mu \mathrm{M}$. For the corresponding analysis of NADPH, measurements were performed at a wavelength of $\lambda=371 \mathrm{~nm}$, because NADPH saturation could not be analyzed at $340 \mathrm{~nm}$ owing to the high absorbance. All kinetic measurements were performed by incubating protein stock solutions containing $0.254 \mu \mathrm{M}$ FAS either with or without $254 \mu \mathrm{M}$ of the $\gamma$ subunit for $30 \mathrm{~min}$ at $30{ }^{\circ} \mathrm{C}$. Single enzymatic reactions contained $12.7 \mathrm{nM}$ FAS with or without $12.7 \mu \mathrm{M} \gamma$-subunit, $0.2 \mathrm{mg} / \mathrm{ml}$ lysozyme as non-specific buffer protein, kinetic assay buffer, the respective required substrate concentrations and 10\% (w/v) sucrose. Single reactions were pre-mixed and incubated $\left(1 \mathrm{~min}, 30^{\circ} \mathrm{C}\right)$. The enzymatic reaction was started by addition of malonyl-CoA and measured at $30{ }^{\circ} \mathrm{C}$. The data were fitted with different kinetic models including the Michaelis-Menten equation, the Hill equation or with a modified Michaelis-Menten equation implementing substrate activation. 


\subsubsection{Biotinylation of FAS}

Biotinylated labeled FAS was used for selection of ScFAS specific nanobodies using phage display. 500 ul of $0.3 \mu \mathrm{M}$ FAS in resuspension buffer was incubated with 10-fold excess (3 $\mu \mathrm{M}$ ) Biotin-dPEG ${ }^{\mathrm{TM}}(24)-\mathrm{NHS}$ (Iris Biotech) at $25{ }^{\circ} \mathrm{C}$ for 30 minutes. The goal was to have single labelled FAS and 10-fold excess of NHS-biotin was found to give the most optimal results. The reaction was quenched by $10 \mu \mathrm{l}$ of $1 \mathrm{M}$ Tris $\mathrm{pH}$ 7.0. The mixture was loaded onto a PD-10 column to separate the unbound biotin. The eluent was collected as $500 \mu \mathrm{l}$ fractions and the ones containing FAS were determined using a dot blot. In the end, $1 \mathrm{ml}$ of $0.12 \mu \mathrm{M}$ biotinylated FAS was obtained.

\subsubsection{Crosslinking Mass Spectrometry}

We analyzed purified FAS holoenzyme by crosslinking mass spectrometry. For BS3 crosslinking, the protein complex was incubated with $1 \mathrm{mM}$ of $\mathrm{BS}^{3}$ crosslinker (from a $100 \mathrm{mM}$ stock in DMSO) for $30 \mathrm{~min}$ at $30^{\circ} \mathrm{C}$. The reaction was quenched with $100 \mathrm{mM}$ Tris$\mathrm{HCl} \mathrm{pH} 8.0$ (from a $1 \mathrm{M}$ stock). For SDA crosslinking, the protein complex was incubated with $2 \mathrm{mM}$ SDA (100 $\mathrm{mM}$ stock in DMSO) for $30 \mathrm{~min}$ at $30^{\circ} \mathrm{C}$, quenched with $50 \mathrm{mM}$ Tris$\mathrm{HCl}$ and dialyzed against reconstitution buffer via a membrane filter (MF Membrane Filters, $0.025 \mu \mathrm{m}$ VSWP, Merck). Afterwards, sample was irradiated with UV light (365 nm wavelength) for $5 \mathrm{~min}$ at $4^{\circ} \mathrm{C}$. For EDC crosslinking, the complex was incubated with $5 \mathrm{mM}$ EDC (100 mM stock in reconstitution buffer) and $10 \mathrm{mM}$ sulfo-NHS for intermediate stabilization for $1 \mathrm{~h}$ at $30^{\circ} \mathrm{C}$. The reaction was quenched with $20 \mathrm{mM}$ dithiothreitol (DTT) and $50 \mathrm{mM}$ Tris- $\mathrm{HCl}$. Proteins were reduced and alkylated with $10 \mathrm{mM}$ DTT and $40 \mathrm{mM}$ iodoacetamide, respectively. In the presence of $1 \mathrm{M}$ urea, proteins were digested by trypsin in an enzyme-to-protein ratio of $1: 50$ at $37^{\circ} \mathrm{C}$ overnight. Peptides were acidified with trifluoroacetic acid (TFA) added to a final concentration of $0.5 \%(\mathrm{v} / \mathrm{v})$, desalted on MicroSpin Colums (Harvard Apparatus) following the manufacturer's instructions, and vacuum dried. Peptides were resuspended in $50 \mu \mathrm{L}$ of $50 \%$ acetonitrile/0.1\% (v/v) TFA before enriching of cross-linked species by peptide size exclusion (SuperdexPeptide 3.2/300 column, GE Healthcare). Fractions of $50 \mu \mathrm{L}$ were collected and those that eluted first and contained the cross-linked peptide pairs were analyzed by LC-MS/MS. 
Cross-linked peptides of the first $\mathrm{BS}^{3}$ replicate were measured in technical duplicates on an Orbitrap Fusion Lumos Tribrid Mass Spectrometer coupled to a Dionex UltiMate 3000 UHPLC system (both Thermo Fisher Scientific) equipped with an in-house-packed $\mathrm{C}_{18}$ column (ReproSil-Pur $120 \mathrm{C} 18-\mathrm{AQ}, 1.9 \mu \mathrm{m}$ pore size, $75 \mu \mathrm{m}$ inner diameter, $30 \mathrm{~cm}$ length, Dr. Maisch $\mathrm{GmbH})$. Samples were separated by applying the following gradient: mobile phase A consisted of $0.1 \%$ formic acid (v/v), mobile phase B of $80 \%$ acetonitrile/ $0.08 \%$ formic acid $(\mathrm{v} / \mathrm{v})$. The gradient started at $5 \% \mathrm{~B}$, increasing to 12,15 or $20 \%$ B within 3 min (according to fraction), followed by a continuous increase to $48 \% \mathrm{~B}$ within $45 \mathrm{~min}$, then keeping B constant at $90 \%$ for $8 \mathrm{~min}$. After each run the column was again equilibrated to $5 \% \mathrm{~B}$ for $2 \mathrm{~min}$. The flow rate was set to $300 \mathrm{~nL} / \mathrm{min}$. MS1 survey scans were acquired in the orbitrap (OT) with a resolution of 120,000, an injection time (IT) of $60 \mathrm{~ms}$ and an automatic gain control (AGC) target of $5 \times 10^{5}$. Dynamic exclusion (DE) was set to $10 \mathrm{~s}$ and only charge states between 3 and 8 were considered for fragmentation. MS2 spectra were acquired in the OT of the 20 most abundant precursor ions, resolution 30,000, IT 120 ms and AGC target $5 \times 10^{4}$. Fragmentation was enforced by higher-energy collisional dissociation (HCD) at $30 \%$ normalized collision energy (NCE). The second $\mathrm{BS}^{3}$ replicate was treated with the following changes: we used a Q Exactive HF-X mass spectrometer (Thermo Fisher Scientific), at MS1 the IT was set to $50 \mathrm{~ms}$ and the AGC target to $1 \times 10^{6}$. At MS2, the IT was set to $128 \mathrm{~ms}$ and the AGC target to $1 \times 10^{5}$. DE covered $30 \mathrm{~ms}$. Here, the 30 most abundant precursor ions were considered for fragmentation. The SDA and EDC sample sets were measured in technical duplicates on an Orbitrap Fusion Tribrid Mass Spectrometer (Thermo Fisher Scientific) with the same settings as described for the first $\mathrm{BS}^{3}$-replicate.

ProteomeDiscoverer 1.4 (Thermo Fisher Scientific) was used for converting .raw files into .mgf format (signal-to-noise ratio 1.5, 1000-10000 Da precursor mass). The generated .mgf files were subjected to pLink v. 1.23(Yang et al., 2012) to identify cross-linked peptides. Here, default settings were applied with carbamidomethylation of cysteines as a fixed and oxidation of methionines as a variable modification, the FDR was set to 0.01 . The crosslinker was selected according to the respective experiment. Spectra of inter-cross-links between FAS and the $\gamma$-subunit within the first BS ${ }^{3}$-replicate as well as for the SDA and EDC sample sets were evaluated manually. For all crosslinkers, a score cut-off of 3 was applied and only 
cross-links supported by more than one crosslinked peptide spectrum matches (CSMs) were considered.

\subsubsection{EM sample preparation and data collection}

\subsubsection{Negative stain grid preparation}

Negative staining is a quick and easy method to visualize proteins in electron microscopy (EM). A heavy metal solution such as uranyl formate is used to stain the proteins. The contrast created due to the differences in electron densities of the stain and the proteins is exploited to image the proteins. First, the protein sample with a concentration of $0.5 \mathrm{mg} / \mathrm{ml}$ is placed in a teflon well (Teflon is inert and does not interact with the proteins). Carbon coated mica was then floated on the sample. The mono-atomic carbon layer keeps floating and adsorbs the particles while the mica sinks. After 30 seconds, the carbon layer was fished using a carbon coated copper grid. The particles were then stained by floating the grid on a saturated uranyl formate solution for $1 \mathrm{~min}$ after which excess stain was removed by gently blotting with a tissue followed by drying for $2 \mathrm{~min}$. The proteins were visualized using a CM200 (Philips) electron microscope. Images were taken at a $66 \mathrm{k}$-fold magnification with a maximal electron dose of 20 electrons per $\AA^{2}$. A voltage of $160 \mathrm{kV}$ was used for acceleration of the electrons. Images were recorded with a two-fold binned $4 \mathrm{k} x 4 \mathrm{k}$ CCD camera (TVIPS) resulting in a pixel size of $2.45 \AA$ / pixel.

\subsubsection{Glutaraldehyde concentration for CtFAS GraFix gradients}

Glutaraldehyde concentrations to be used for GraFix were determined by incubating $20 \mu \mathrm{g}$ of CtFAS with increasing concentrations of Glutaraldehyde $(0,0.025,0.05,0.01,0.02 \%)$ in a total volume of $20 \mu \mathrm{l}$ for $30 \mathrm{mins}$ at $4^{\circ} \mathrm{C} .10 \mu \mathrm{l}$ of this was loaded onto an SDS-PAGE gel. The concentration of $0.05 \%$ glutaraldehyde was found to be optimal as the bands corresponding to CtFAS began to migrate slower at this crosslinker concentration. 


\subsubsection{Preparative reconstitution of the FAS $\gamma$-subunit complex}

For reconstitution of the FAS holoenzyme, a 100-fold excess of the $\gamma$-subunit was added to $4 \mu \mathrm{M} F A S$ and incubated at $30^{\circ} \mathrm{C}$ for $30 \mathrm{~min}$. The protein was then loaded on $10-45 \%(\mathrm{w} / \mathrm{v})$ sucrose gradients in purification buffer containing $10 \mathrm{mM}$ DTT, and then centrifuged at $64,000 \mathrm{x}$ f for $16 \mathrm{~h}$ at $4{ }^{\circ} \mathrm{C}$. Gradients were harvested in $400 \mu \mathrm{l}$ fractions. We then used SDSPAGE to identify fractions containing FAS holoenzyme. Selected fractions were pooled and precipitated by the addition of $40 \%(\mathrm{v} / \mathrm{v})$ PEG400. After centrifugation $(30,000 \times \mathrm{g}, 30$ minutes), the supernatant was removed and the precipitate was resuspended in purification buffer containing 10\% (w/v) sucrose, $10 \mathrm{mM}$ DTT and $0.01 \%(\mathrm{w} / \mathrm{v})$ LMNG to a final concentration of $10 \mathrm{mg} / \mathrm{ml}$.

\subsubsection{Cryo-EM sample preparation}

\section{CtFAS}

For the native CtFAS dataset, 200 pmol of purified CtFAS was loaded on $10-45 \%(\mathrm{w} / \mathrm{v})$ linear sucrose gradient in purification buffer containing $10 \mathrm{mM}$ DTT and centrifuged at $100,000 \times \mathrm{g}$ for $16 \mathrm{~h}$ at $4{ }^{\circ} \mathrm{C}$. The gradient was harvested in $200 \mu \mathrm{l}$ fractions and those containing the FAS were identified using dot blots stained with $0.1 \%$ amido black solution. The FAS fraction were pooled and buffer exchanged to purification buffer lacking sucrose using a Zeba spin column (Thermo Scientific, Germany). The particles were adsorbed to a continuous carbon film attached to a Quantifoil (3,5/1) (Quantifoil, Jena, Germany) grid for 2 min at $4{ }^{\circ} \mathrm{C}$. The grid was then transferred to a Vitrobot Mark IV (Thermofisher, Germany) plunge-freezer, where it was vitrified after blotting for $8 \mathrm{~s}$ at $4{ }^{\circ} \mathrm{C}$ and $100 \%$ humidity.

For the GraFix treated CtFAS, the above protocol had two variations. The sucrose gradients also contained a linear $0-0.05 \%$ glutaraldehyde gradient and $20 \mathrm{mM}$ sodium aspartate $(\mathrm{pH}$ 6.5) was added to all the harvested gradient fractions to quench the crosslinking reaction before proceeding. 


\section{ScFAS}

EM grids were prepared for ScFAS, ScFAS- $\gamma$-subunit complex and ScFAS with substrates (1 mM Malonyl-CoA and $2 \mathrm{mM} \mathrm{NADPH}$ ) at a protein concentration of $0.5 \mathrm{mg} / \mathrm{ml}$. The particles were adsorbed to a continuous carbon film attached to a Quantifoil (3,5/1) (Quantifoil, Jena, Germany) grid for $2 \mathrm{~min}$ at $4{ }^{\circ} \mathrm{C}$. The grid was then transferred to a Vitrobot Mark IV (Thermofisher, Germany) plunge-freezer, where it was vitrified after blotting for $8 \mathrm{~s}$ at $4{ }^{\circ} \mathrm{C}$ and $100 \%$ humidity.

For studying the effect of $\gamma$-subunit concentration on the conformation landscape of ScFAS, $\gamma$-subunit concentration was titrated against a constant amount of FAS. For this, $0.5 \mu \mathrm{M}$ FAS solution was used and recombinant, purified $\gamma$-subunit was titrated in $0-, 2-, 4-, 6-, 12-$, 24-, 48- and 96-fold excess. The protein mix was incubated at $30{ }^{\circ} \mathrm{C}$ for $30 \mathrm{~min}$ and then kept at $4{ }^{\circ} \mathrm{C} .4 \mu \mathrm{l}$ of each sample was applied to freshly glow-discharged Quantifoil R1.2/1.3 and R2/2 holey carbon grids (Quantifoil Micro Tools, Jena, Germany) followed by plunge freezing as described above.

For FAS-Nb complexes, $0.5 \mathrm{mg} / \mathrm{ml}$ FAS solution was incubated with 12-fold molar excess of the respective nanobody for 30 mins at RT. The particles were adsorbed to a continuous carbon film and plunge frozen as described above.

\subsubsection{Cryo-EM data collection and image processing}

Data were acquired on a Titan Krios (Thermo Fisher Scientific) operating at $300 \mathrm{kV}$ using either a Falcon III (integrating mode) camera or a K2 summit (counting mode) camera (Gatan, Inc.). Data collection and processing statistics are summarized in Supplementary Table 3 and 5. The acquired movies were motion corrected and dose weighted using Motioncor2 (Zheng et al., 2017) with all the frames split into 5x5 patches. The aligned frames were used to perform per micrograph CTF estimation using GCTF (Zhang, 2016). Particle selection was done using Gautomatch (from Dr. K. Zhang). All subsequent image processing steps were performed with Relion 3.0 (Scheres, 2012; Zivanov et al., 2018), unless otherwise specified (Figure 16, Supplementary Figure. S1, S4). The extracted particles were subjected to 3 rounds of reference free 2D classification to remove bad/empty images. The 
remaining particles were refined with a mask around the central wheel using D3 symmetry. EMDB-1623 was low pass filtered to $30 \AA$ and used as reference for all 3D refinement and 3D classification procedures. The refined particles were then classified without alignment, with a mask around the two domes. The particles from the 3D class displaying the best resolution were selected and used for one more round of 3D refinement and classification as performed before. The data further classified into 3 main classes. The particles belonging to their respective classes were refined resulting in structures with resolutions of $3.5 \AA$ or better. Even though all the 3 classes were at comparable resolutions, their local resolutions - especially in the dome region of the molecule - were very different. Only one class displayed high resolution details throughout the whole molecule. Finally, the particles belonging to this class were selected and used to perform CTF refinement (and particle polishing for the FAS holoenzyme dataset) followed by 3D refinement.

\subsubsection{Energy Landscapes calculation}

The data were processed as described above until the first classification step. For this analysis, batches of $\sim 100,000$ particles were classified into 15 classes. The particles belonging to each class were selected and refined again without applying any masks. The refined 3D volumes from each dataset (a total of 165) were then used for the conformational landscape analysis as previously described (Haselbach et al., 2017, 2018). The 3D volumes were first aligned in USCF Chimera (Pettersen et al., 2004) with the central wheel of the molecule as the reference point. Further steps were performed in the COW software suite (www.cowem.de). The 3D volumes were normalized and filtered to $20 \AA$. 3D Principle Component Analysis (PCA) was then performed to obtain the major modes of motion among the different samples. The resultant eigenvectors representing the variance in the data were sorted based on decreasing significance and then used to describe each 3D volume using the following equation

$$
X_{i}=\bar{X}+\sum_{j=1}^{n} a_{i, j} e_{i}
$$

, where every volume $X_{i}$ is described as a linear combination of the average volume $\bar{X}$ and the eigenvectors $e_{i}$ multiplied with the linear factor $a_{i, j}$. The first $\left(e_{1}\right)$ and second $\left(e_{2}\right)$ eigenvectors, which depict motions of compaction and rotation of the FAS dome, 
respectively, were selected for calculating conformational landscapes. The particle number belonging to each class was used to calculate their free energies as multiples of the Boltzmann factor $\left(k_{B} T\right)$ :

$$
\Delta \Delta G=k_{B} T \ln \left(\frac{p_{i}}{p_{0}}\right)
$$

, where $T$ is the absolute temperature, $k_{B}$ the Boltzmann constant, $p_{i}$ is the number of particles in state $i$ and $p_{0}$ is the number of particles in the most populated state. The linear factors of $e_{1}$ and $e_{2}$ calculated as per equation (1) contributing to the input 3D volumes represent the $\mathrm{x}$ and $\mathrm{y}$ coordinates of the landscapes whereas the $\mathrm{z}$ axis represents the free energies of each class.

\subsubsection{X-ray crystallography methods}

\subsubsection{Crystallization and Stabilization}

FAS in purification buffer containing 10\% sucrose (w/v), $10 \mathrm{mM}$ DTT and $0.01 \%(\mathrm{w} / \mathrm{v})$ LMNG was crystallized at $7 \mathrm{mg} / \mathrm{ml}$ by mixing $1 \mu \mathrm{l}$ protein $+1 \mu \mathrm{l}$ crystallization buffer $\mathrm{A}$ in Crystalgen hanging drop diffusion plates (Jena Bioscience, Germany) over a $750 \mu \mathrm{l}$ reservoir of crystallization buffer A. For the FAS holoenzyme, a 7-fold molar excess of $\gamma$ subunit (in the buffer used for FAS) was added to a $7 \mathrm{mg} / \mathrm{ml}$ protein solution of FAS and incubated for 30 minutes at $30{ }^{\circ} \mathrm{C}$. The reconstituted complex was then crystallized by mixing $1 \mu \mathrm{l}$ protein $+1 \mu \mathrm{l}$ crystallization buffer B in Chryschem sitting drop vapor diffusion plates (Hampton Research, Aliso Viejo, USA) over a $500 \mu \mathrm{l}$ reservoir of crystallization buffer B. The crystals took 3-7 days to grow at $18{ }^{\circ} \mathrm{C}$ and were usually around $150 \times 200 \times 200 \mu \mathrm{m}$ in size.

The crystals obtained were transferred to $4{ }^{\circ} \mathrm{C}$ by gradually decreasing the temperature in a linear manner over $48 \mathrm{~h}$ using a Rumed ${ }^{\circledR}$ E100 incubator (Rubarth Apparate GmbH, Germany). Stabilization and dehydration procedures were performed as follows: 1) The drop was unsealed and $2 \mu \mathrm{l}$ of the reservoir solution was added to the drop. Then $2 \mu \mathrm{l}$ of crystal stabilization buffer (A for FAS, B for FAS holoenzyme) was added. 2) $2 \mu$ l of crystal stabilization buffer containing $5 \%(\mathrm{v} / \mathrm{v})$ ethylene glycol was added followed by removal of 
$2 \mu \mathrm{l}$ of solution from the drop. Then $2 \mu \mathrm{l}$ of crystal stabilization buffer containing $5 \%(\mathrm{v} / \mathrm{v})$ ethylene glycol was again added to the drop. This procedure was repeated with crystal stabilization buffer containing $10 \%$ and then $20 \%(\mathrm{v} / \mathrm{v})$ ethylene glycol. 3) The reservoir solution was exchanged for crystal stabilization buffer containing 25\% (v/v) ethylene glycol and the drop was resealed. The crystals were then allowed to equilibrate by vapour diffusion in excess of $16 \mathrm{~h}$.

\subsubsection{X-ray Diffraction Data collection}

The crystals were harvested in Spine Litholoops (Molecular Dimensions, Suffolk, UK or Jena Bioscience, Jena, Germany) mounted on magnetic pins, and vitrified by plunge-cooling in liquid nitrogen. The data were collected on EMBL beamline P14 at the PETRA III storage ring (DESY, Hamburg, Germany) using a MD3 vertical-spindle diffractometer (EMBL and Arinax, Moirans, France) and an EIGER 16M detector (Dectris, Baden, Switzerland). A white beam refractive lens transfocator and slits were used to obtain homogenous X-ray beams which matched the crystal dimensions (Schrader et al., 2016). Diffraction data were scaled and integrated with the XDS program package (Kabsch, 2010).

\subsubsection{X-ray structure determination}

Initial phases for FAS were determined by molecular replacement performed with MOLREP (Vagin and Teplyakov, 2010) using the Saccharomyces cerevisiae FAS structure (PDB ID: 2UV8). The model was built and optimized by several rounds of interactive manual model building in Coot (Emsley and Cowtan, 2004) and refinement in Refmac5 (Murshudov et al., 2011). Finally, TLS refinement was performed, where each TLS domain was defined by individual enzymatic domains. The obtained structure displays excellent stereochemistry with $19.7 \%$ / 21.8\% (R/R free) (Supplementary Table 4). Analysis of the diffraction data with the STARANISO server indicated mild anisotropy. This suggested anisotropic scaling and truncation of the data should allow for better refinement of the model. This was indeed the case and resulted in a final model with lower average B-factors and $19.2 \%$ / $21.1 \%(\mathrm{R} /$ Rfree) (Supplementary Table 4). 
The refined FAS crystal structure was used to determine the initial phases by molecular replacement with MOLREP (Vagin and Teplyakov, 2010) for the FAS-holoenzyme diffraction data. The obtained density map was found to correlate better with the FAS holoenzyme model (as determined by cryo-EM) rather than the FAS structure. Therefore, we used the model of $\alpha$ - and $\beta$-subunits from the FAS holoenzyme cryo-EM model in initial refinement of the X-ray data. Notably, the ACP domain and the $\gamma$-subunit were removed from the structure prior to refinement to avoid introducing a phase bias. After several rounds of rigid body refinement in Refmac5 (Murshudov et al., 2011), difference densities corresponding to the ACP domain and $\gamma$-subunit next to the AT and ER domain, respectively could be easily identified. The ACP and the $\gamma$-subunit sequences were then placed into their respective densities followed by additional rounds of refinement using Coot (Emsley and Cowtan, 2004) and Refmac5 (Murshudov et al., 2011).

\subsubsection{EM Model building}

The crystallographic FAS model was used as the initial model for both the FAS and FAS holoenzyme structures determined by cryo-EM. It was docked into the EM densities as a rigid body using UCSF Chimera (Pettersen et al., 2004). An additional round of rigid body refinement was then performed in Refmac5 (Murshudov et al., 2011). The model then underwent several rounds of manual modelling in Coot (Emsley and Cowtan, 2004) and refinement in Refmac5 (Murshudov et al., 2011) (Supplementary Table 3).

Several models for the $\gamma$-subunit were generated based on sequence homology using the Robetta (Kim et al., 2004) prediction server. All of them were docked into the "extra density" in the FAS holoenzyme map using UCSF Chimera (Pettersen et al., 2004). The model with the best fit was then manually modelled into the density based on the crosslinking data as well as side chain densities that were visible. There were two unstructured regions $(60-76,114-132)$ of this protein that could not be confidently modelled.

For validation of the models, fourier shell correlation (FSC) between the refined model and

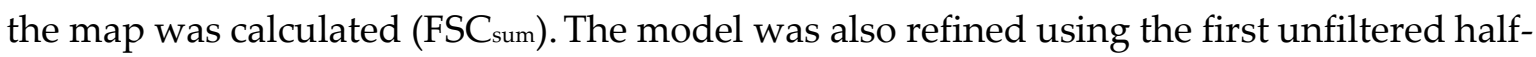
map. This model was then compared to the first half map (FSCwork) as well as to the second half-map ( FSC $_{\text {free }}$ ) to check for signs of overfitting. 
All figures were made using Pymol (Schrödinger, 2015), Chimera (Pettersen et al., 2004) and ChimeraX (Goddard et al., 2018). 


\section{Results}

The first step to fulfill the aims set for this thesis was to obtain a preparation of compositionally homogenous fungal FAS. Previous structural studies of this complex mainly relied on the use of ammonium sulfate precipitation, sucrose gradients and anion exchange chromatography to purify endogenous FAS (Jenni et al., 2007; Leibundgut et al., 2007; Lomakin et al., 2007). Some research groups have also tried to use recombinant approaches by either tagging the endogenous gene with a His-Tag or expressing the full molecule in E. coli (Elad et al., 2018; Johansson et al., 2008). Using these purification methods, 3.1-3.3 $\AA$ structures of type I FAS have been solved from fungi Thermomyces lanuginosus and Saccharomyces cerevisiae along with those from pathogenic bacteria like Mycobacterium tuberculosis. To be able to study the FAS structure and dynamics at high resolution, I tested a different approach involving PEG precipitation and sucrose gradients, while maintaining constant low salt conditions $(<100 \mathrm{mM})$. A similar mild purification strategy was successfully applied for purifying 20S/26S proteasomes (Haselbach et al., 2017; Schrader et al., 2016) and enabled the study of these structures at high resolutions.

\subsection{Structure investigations of Chaetomium thermophilum FAS}

\subsubsection{Purification of endogenous FAS from Chaetomium thermophilum}

A chromatography-free purification of endogenous FAS from Chaetomium thermophilum (CtFAS) was established based on the method previously employed to purify 20/26S proteasomes (Haselbach et al., 2017; Schrader et al., 2016) (Figure 5). Cell lysate prepared from frozen ground cells was centrifuged at 30,000g for $30 \mathrm{~min}$ to remove cell debris followed by centrifugation at 100,000g for $1 \mathrm{hr}$. FAS was then precipitated from the clarified lysate using 15\% PEG400. The precipitated proteins were then loaded onto 10-45\% sucrose gradients (Figure 5(ii)). The fractions containing FAS were pooled and then precipitated using $6 \%, 8 \%$ and $10 \%$ PEG3350 concentrations. The 8-10\% PEG3350 cut contained the FAS and was then loaded onto 10-45\% sucrose gradients (Figure 5(iii)). The fractions containing the FAS were pooled, precipitated and resuspended to a concentration of $12 \mathrm{mg} / \mathrm{ml}$. FAS 
bands of 210 and $230 \mathrm{kDa}$ were confirmed using mass spectrometry. Along with this, the major impurity in the sample were two bands around $100 \mathrm{kDa}$ which were identified as subunits of the oxoglutarate dehydrogenase complex. The oxoglutarate dehydrogenase complex is in the same molecular weight range as the FAS and therefore always cosediments with the FAS. Optimization of PEG precipitation steps to remove the oxoglutarate dehydrogenase complex from the FAS preparations was tested but was unsuccessful. Therefore, even with the present impurities, I used the CtFAS sample for negative stain electron microscopy to check whether the purity of the sample was adequate for structural studies.

(i)

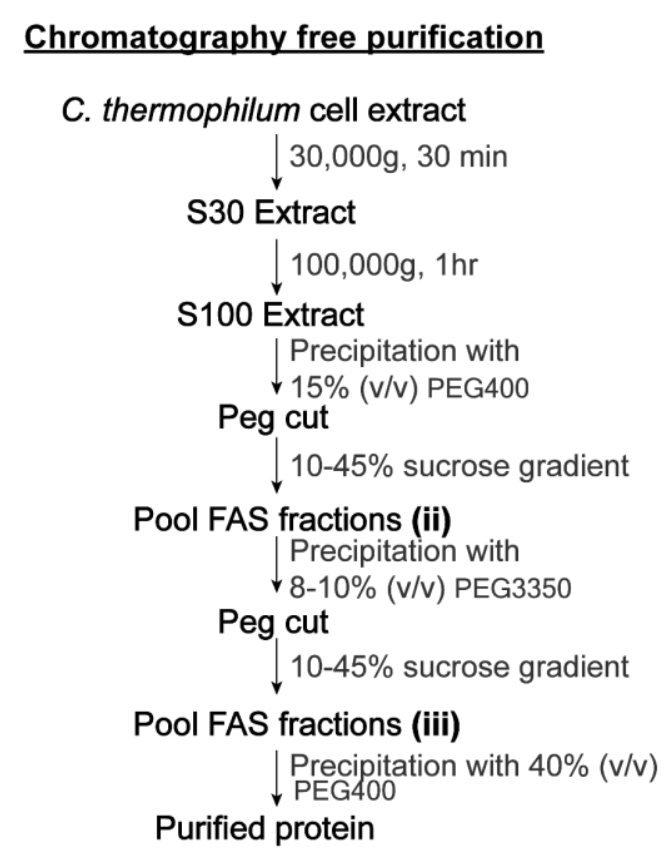

(ii)

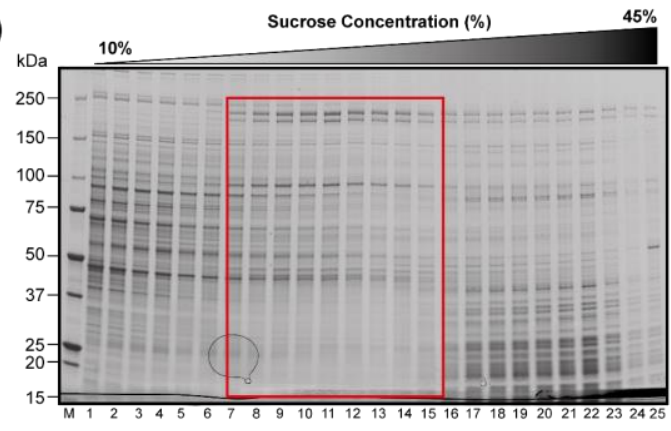

(iii)

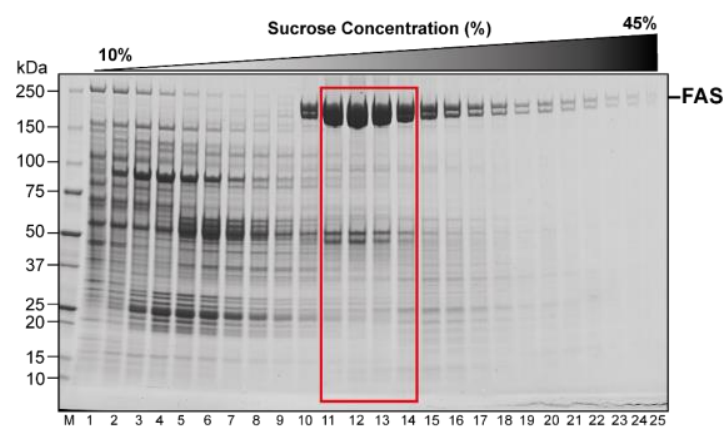

Figure 5. Schematic representation of the chromatography-free purification established for Chaetomium thermophilum FAS. (i) The chromatography-free purification of FAS involved specific precipitation of FAS using PEG in combination with sucrose density gradients. The SDS$P A G E$ gel for the fractions of the first (ii) and second (iii) 10-40\% sucrose gradient step is depicted. Fractions containing the FAS that were pooled are indicated by the red box. 


\subsubsection{Negative stain electron microscopy}

The purified endogenous CtFAS was first analyzed with negative stain EM to check the purity of the sample. Visually, the obtained micrographs consist mainly of FAS particles $(>90 \%)$ along with some particles that correspond to the oxoglutarate dehydrogenase complex as well as unexpected $20 \mathrm{~S}$ proteasomes (Figure 6(i)). This observation correlated with the amount of contaminants observed by SDS-PAGE during purification. 2D class averages of the selected particles depicted classical side views of a type I fungal FAS (Figure 6(i)). The 2D class averages were used for generating a de novo 3D starting model using the COW suite (Figure 6(ii)). I then used Relion 2.0 (Scheres, 2012) for refining the particles using the de novo 3D model as a reference. This resulted in a $20 \AA$ structure which, at this resolution, appears to be identical to other published fungal FAS structures (Figure 6(iii)). After confirming that the purified CtFAS was pure and intact for structural studies, I used the sample for crystallization as well as cryo-EM in order to determine the structure at high resolution.

(i)

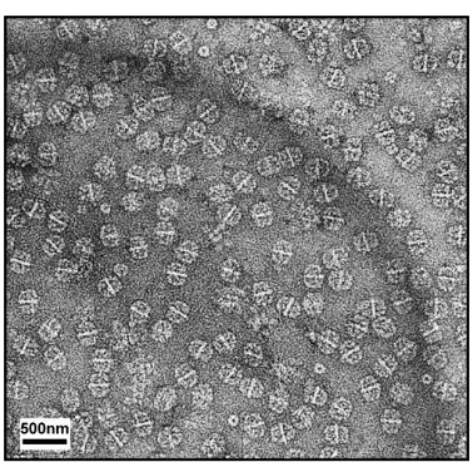

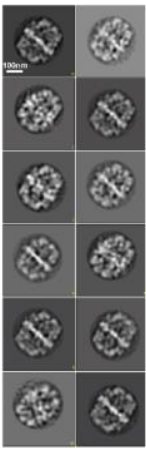

(ii)

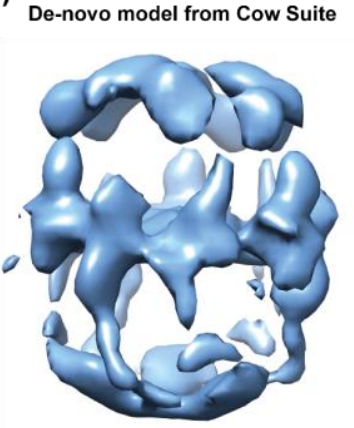

(iii)

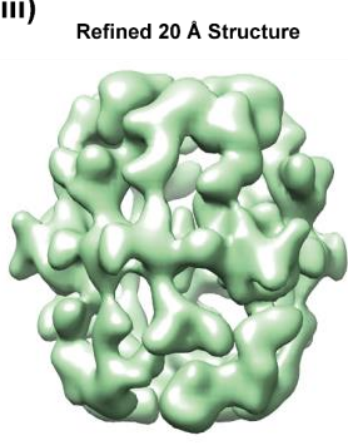

Figure 6. Analysis of CtFAS using negative stain electron microscopy. (i) Negative stain micrograph of the purified CtFAS along with representative $2 D$ class averages. (ii) De novo $3 D$ starting model of the CtFAS reconstructed using COW suite. (iii) 20 A negative stain EM structure of the CtFAS. 


\subsubsection{Crystallization trials}

Crystallization of CtFAS was done in parallel with the cryo-EM studies. The strategy for screening crystallization conditions involved testing different combinations of precipitant and salt concentrations. Crystals in the range of $150-300 \mu \mathrm{m}$ were obtained at $18{ }^{\circ} \mathrm{C}$ using two crystallization conditions in 0.1M MES pH 7.0: (1) $0.6 \mathrm{M} \mathrm{KCl,} \mathrm{6-7 \%} \mathrm{PEG} 6000$ and (2) $1 \mathrm{M}$ ammonium nitrate, 7-8\% PEG5000. The crystals belonged to C2221 space group with a unit cell of $\mathrm{a}=264.2 \AA, \mathrm{b}=351.2 \AA, \mathrm{c}=418.4 \AA, \alpha=\beta=\gamma=90^{\circ}$ and the best crystals diffracted up to $\sim 5-6 \AA$ A. Furthermore, the crystal diffraction was more reproducible upon transfer to 4 ${ }^{\circ} \mathrm{C}$ before stabilization and dehydration procedures. Due to the crystal diffraction being limited to low-resolutions of the obtained crystals, I exclusively used cryo-EM to study the effects of GraFix on CtFAS structure.

\subsubsection{Cryo-EM structure determination of CtFAS}

Cryo-EM data for the CtFAS were acquired on a Titan Krios with a Cs corrector at $300 \mathrm{kV}$ using a Falcon II direct electron detector in integrating mode. The micrographs displayed good contrast, which enabled robust particle selection. I first performed 2D classification using 492,557 particles selected from 6421 micrographs (Figure 7(i)). After each 2D classification step, empty images or images with low signal-to-noise were discarded. The obtained 2D class averages consisted exclusively of side views of the FAS. This was followed by 3D classification procedures in Relion 3.0 which further sorted the data into one good subset of particles (73,391 particles, $14.9 \%$ of total particles). This final set of particles resulted in a $3.2 \AA$ density map with applied D3 symmetry (Figure 7(ii)). Even though the FSC 0.143 criterion suggested the map resolution to be $3.2 \AA$, local resolution variations in the structure were present, from $\sim 3 \AA$ at the central wheel to $\sim 3.4-3.8 \AA$ at the dome region. Furthermore, the angular distribution of the particles used for the 3D refinement displayed an orientation bias (Figure 7(iii)). In the angular distribution plot, a tilt angle of $90^{\circ}$ represents the side view of the FAS whereas $0^{\circ}$ represents the top view of the FAS. The data consists mainly of side views of the FAS along with minor populations of particles adopting tilted orientations, however, none of the particles show top views of 
the complex. Such an orientation distribution is not ideal but was sufficient to obtain a $3.2 \AA$ structure of CtFAS. A similar view distribution of an asymmetric complex would result in more apparent effects on the obtained structure such as smearing of the density in the direction of the major particle projection orientation. Whether such an orientation bias is limiting the attainable resolution for the CtFAS is an open question and I tried to answer this using the GraFix procedure as described in the next section.

To build an atomic model into the cryo-EM map, previous high resolution FAS structures from Thermomyces lanuginosus and Saccharomyces cerevisiae were first fit into the map at the secondary structure level. Both structures correlate well with the map, with all domains except ACP and PPT modelled in the map. In the end, the Thermomyces lanuginosus structure was found to be a more suitable template for building the CtFAS structure as CtFAS has a sequence identity of $\sim 74 \%$ with the T. languinosus FAS as compared to $\sim 60 \%$ with the $S$. cerevisiae FAS (ScFAS). After mutating the respective residues, I first refined the model interactively followed by refinement using Refmac5. The final refined model of the CtFAS had a FSC $0.5=3.3 \AA$ and a cross-correlation of 0.76 with the CtFAS map (Figure 7(iv)). 
(i)

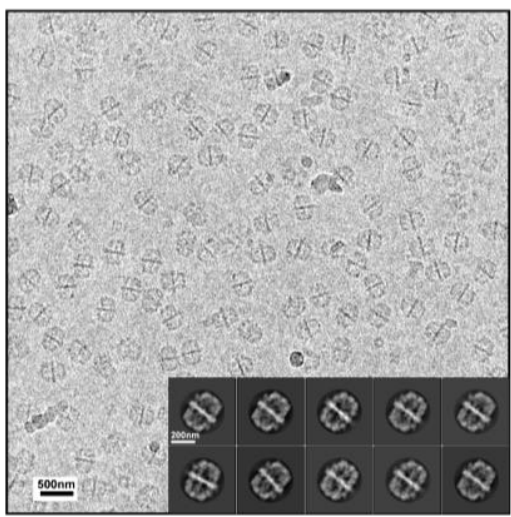

(iii)

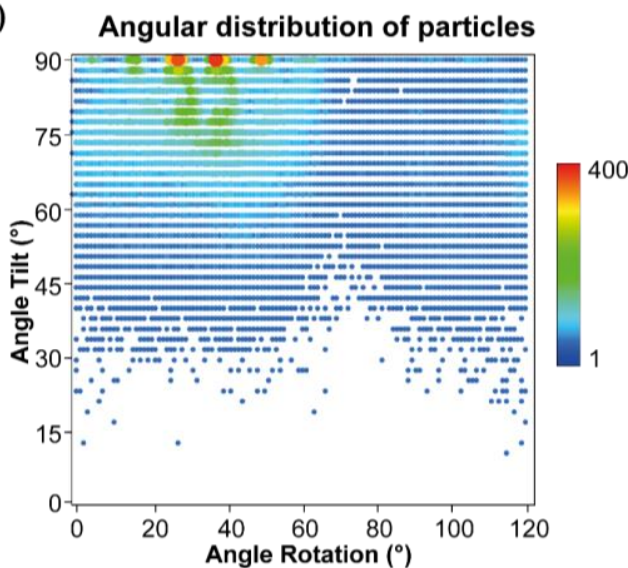

(ii)

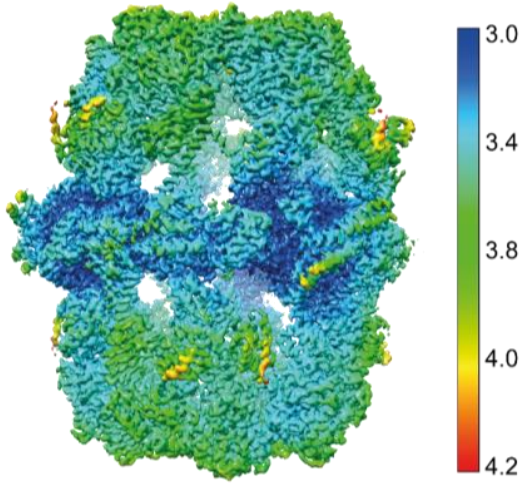

(iv)

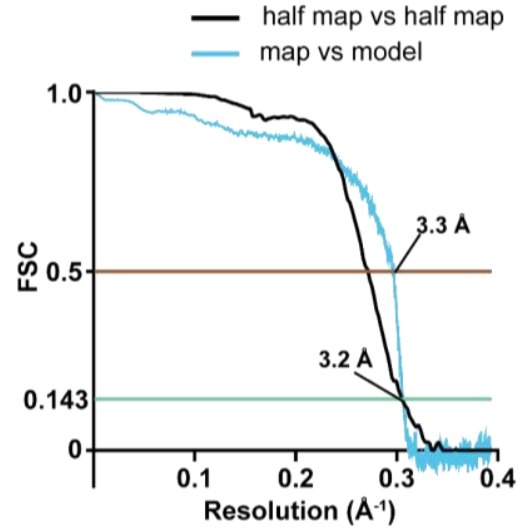

Figure 7. Cryo-EM analysis of CtFAS. (i) A typical electron micrograph of CtFAS embedded in vitreous ice along with representative $2 D$ class averages. (ii) Local resolution map of the $3.2 A C t F A S$ structure. The local resolution varies from $3 \AA$ at the central wheel to 3.4-3.8 $\AA$ at the dome. (iii) Angular distribution of particles used in the final 3D reconstruction. (iv) Fourier shell correlation (FSC) curves of two independently refined subsets of particles (half map vs half map) as well as the final refined map and the model (map vs model) are shown. The half map vs half map curve indicates a resolution of $3.2 \AA$ at a threshold of 0.143 . The map vs model curve shows a resolution of $3.3 \AA$ using the 0.5 threshold criterion.

The $3.2 \AA$ structure of CtFAS is the highest resolved structure of a type I fungal FAS determined by cryo-EM and is comparable to the $3.1 \AA$ structures determined previously by X-ray crystallography. Multiple 3D classification steps were performed to further improve the resolution, however, the resolution of the CtFAS structure did not improve. The attainable resolution for a biological sample with the microscope used at the time this data was acquired was better than $2.5 \AA$ (data not shown). This indicated that the resolution limiting factor was most likely not the structural technique used, but the heterogeneity of the protein sample itself. One factor contributing towards this could be the unresolved ACP 
domain. The ACP domain interacts with six catalytic domains within the FAS dome during fatty acid synthesis. It can therefore be either located at one (or many) of the catalytic domains or can be present in the empty space inside in the FAS dome. The ACP domain not being visible in the CtFAS structure suggests that the domain is not stabilized at a distinct site in the CtFAS. Thus, the random distribution of ACP domains would explain the absence of any corresponding density within the FAS as their signal gets averaged out during $3 \mathrm{D}$ reconstruction. In the FAS, since the ACP domains are tethered to the FAS barrel with linker sequences, it is plausible that the inconsistent distribution of the ACP domain within the FAS results in random variations in the FAS barrel itself.

To address the issue of conformational heterogeneity and to improve the resolution of the CtFAS structure, I tested the GraFix method (Kastner et al., 2008). The hypothesis was that this mild crosslinking method could assist in stabilizing the FAS dome and the ACP domain within.

\subsubsection{Effect of Grafix on CtFAS structure.}

As a crosslinking agent for GraFix, I used glutaraldehyde. Glutaraldehyde is a zero-length bi-functional crosslinker, which can covalently link closely situated primary amine groups in protein complexes. Glutaraldehyde could affect the FAS structure in two ways, (1) by intramolecular crosslinks between closely situated lysine residues and (2) by changing the charge distribution on the surface of the molecule using a charged molecule to quench the monovalently attached glutaraldehyde molecules. I tested the optimal concentration of glutaraldehyde for GraFix by in-batch crosslinking at $4{ }^{\circ} \mathrm{C}$ and found that $\sim 0.05 \%$ glutaraldehyde results in mild crosslinking of the FAS subunits as they migrate slower than the untreated FAS. At higher glutaraldehyde concentrations the FAS subunits are completely crosslinked rendering them too large to enter the SDS-PAGE gel (Figure 8(i)). I then used $0.05 \%$ of glutaraldehyde for mild crosslinking of CtFAS using GraFix and the crosslinking reaction was terminated using $20 \mathrm{mM}$ aspartate $\mathrm{pH}$ 6.5. GraFix-treated FAS appeared to be more thermostable as shown by the Proteoplex assay (Chari et al., 2015) (Figure 8 (ii)). The increase in melting temperature upon GraFix was most likely a result of intramolecular crosslinks. Along with this, negative stain micrographs of the GraFix-treated 
FAS displayed particles with equally distributed top and side views of the complex (Figure 8(iii)). This was distinctly different from the untreated FAS where only side views were visible (Figure 6(i)). The use of aspartate for quenching the GraFix reaction makes the FAS more negatively charged, which is the most likely reason for the change in particle orientations.

(i)

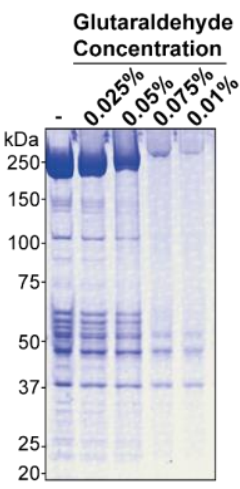

(ii)

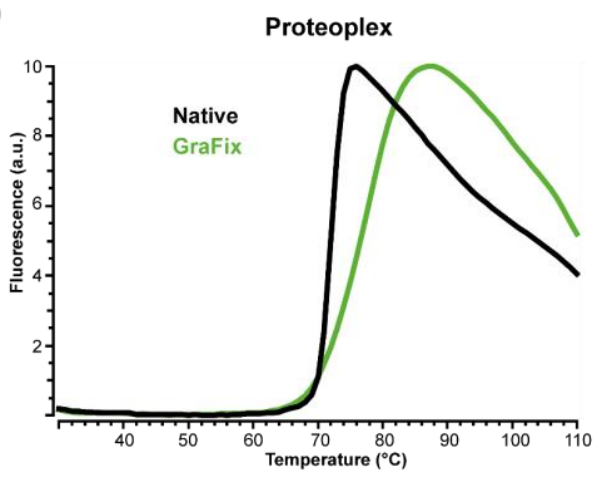

(iii)

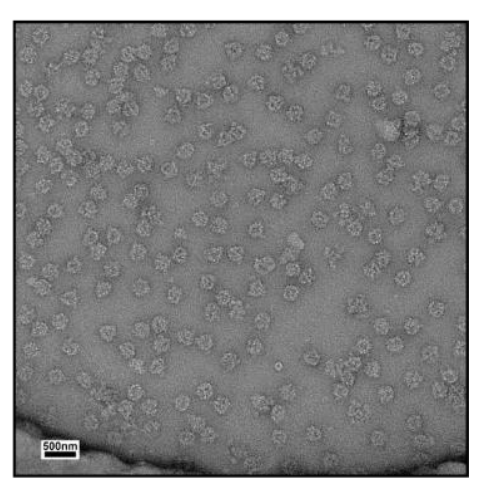

Figure 8. GraFix treatment of the CtFAS. (i) Gel depicting the effect of increasing glutaraldehyde concentration on the migration of FAS subunits (210, $230 \mathrm{kDa}$ ) on SDS-PAGE. Mild crosslinking is observed at $0.05 \%$ glutaraldehyde due to slower migration of the FAS subunits whereas complete crosslinking is observed at $0.075 \%$ onwards as the FAS subunits are unable to enter the gel. (ii) Normalized melting curves of the untreated (black) and GraFix-treated (green) FAS using the Proteoplex assay (Chari et al., 2015). (iii) Typical negative stain micrograph of GraFix-treated CtFAS.

Finally, I used cryo-EM to determine if GraFix had implications for high resolution determination of CtFAS. The data was collected on a Titan Krios with a Cs corrector at 300 kV using a Falcon II direct electron detector in integrating mode. A set of 573,431 particles were selected from 6496 micrographs and used for 2D classification. The 2D class averages were different from those obtained with untreated FAS (Figure 9(i)). Along with the side views, tilted views of the FAS were also present. Furthermore, upon 3D classification, I found that GraFix-treated CtFAS was conformationally similar to the untreated FAS with most particles belonging to one major state of the FAS. Further, no densities corresponding to the ACP domain were observed during the different image processing steps. After multiples round of 3D classification, 110,961 particles were selected and used to reconstruct a 3.4 A GraFix-treated FAS structure with applied D3 symmetry (Figure 9 (ii)). 
(i)

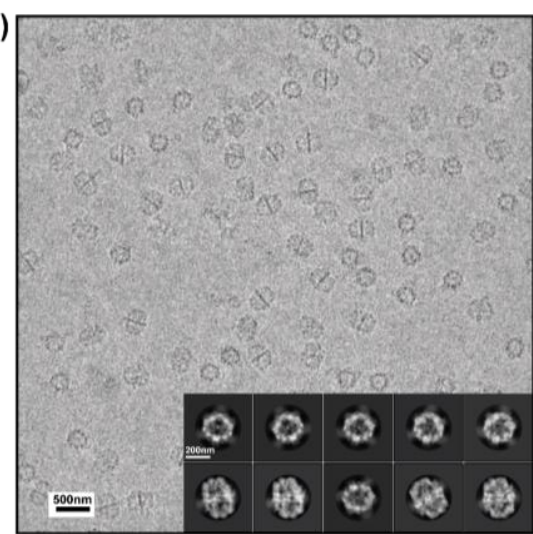

(iii)

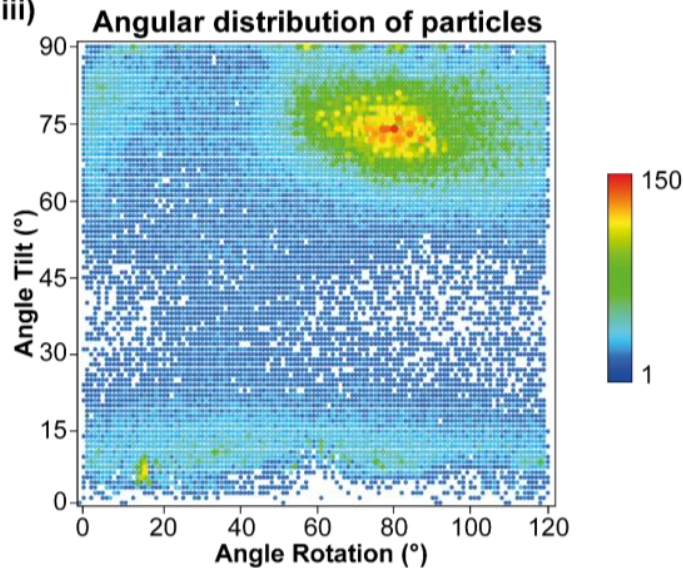

(ii)

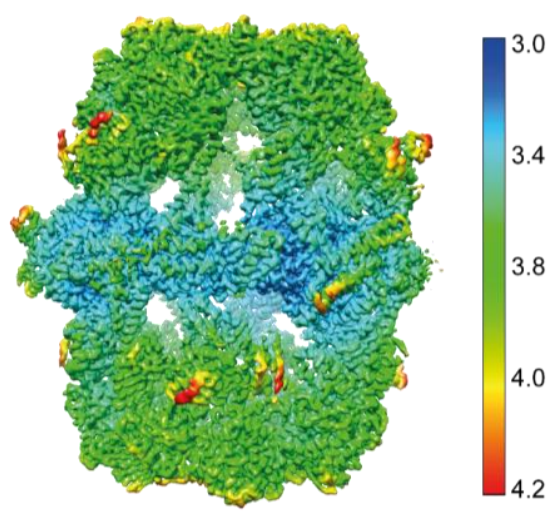

(iv)

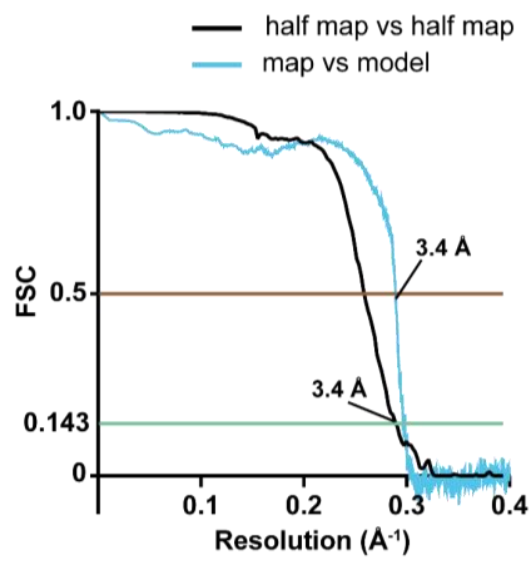

Figure 9. Cryo-EM analysis of GraFix-treated CtFAS. (i) A typical electron micrograph of the GraFix-treated CtFAS embedded in vitreous ice along with representative $2 D$ class averages. (ii) Local resolution map of the $3.4 \AA$ CtFAS structure. The local resolution varies from 3-3.4 $\AA$ at the central wheel to 3.8-4 $\AA$ at the dome. (iii) Angular distribution of particles used in the final $3 D$ reconstruction of $3.4 \AA$. (iv) Fourier shell correlation (FSC) curves of two independently refined subsets of particles (half map vs half map) as well as the final refined map and the model (map vs model) are shown. The half map vs half map curve indicates a resolution of $3.4 \AA$ at a threshold of 0.143. The map vs model curve shows a resolution of $3.4 \AA$ using the 0.5 threshold criterion.

The untreated FAS was resolved at $3.2 \AA$, which was better by $0.2 \AA$ as compared to the GraFix-treated FAS. Along with no discernible effect on the structure of the FAS, the mild fixation condition tested was ineffective in stabilizing the ACP domain. However, upon GraFix, particle orientations are more uniformly distributed as compared to the untreated FAS, where one view of the particle is over-represented (Figure 9(iii)). This is apparent both in the $2 \mathrm{D}$ class averages as well as in the angular distribution of the particles used for $3 \mathrm{D}$ 
refinement. To further assess the effect of GraFix on the FAS structure, I refined the CtFAS model obtained from the untreated FAS map against the GraFix-treated FAS map (Figure 9 (iv)). The final refined model of the GraFix-treated CtFAS had a FSC $0.5=3.4 \AA$ and a crosscorrelation of 0.87 with the map (Figure 9(iv)).

On comparing the untreated and GraFix-treated FAS models, no structural differences were observed and a root-mean-square deviation (RMSD) between the C $\alpha$ atoms of the two models $\sim 0.4 \AA$, suggesting that the two structures are almost identical. Thus, even though GraFix stabilized the molecule, as indicated by the Proteoplex assay, it had no apparent impact on the structure of the CtFAS or on the attained resolution. However, GraFix treatment aided in achieving a uniform particle orientation distribution of the FAS.

The combination of glutaraldehyde for crosslinking and aspartate as a quenching agent used here for GraFix can be further modified in order to find conditions conducive to structural stabilization of the FAS dome, the ACP domains or both. For this purpose, chemical crosslinkers with different length or reactivity towards different function groups, in combination with quenching agents with different charge properties can be tested. However, for the rest of the thesis, I changed the model organism to Saccharomyces cerevisiae taking into account the following two observations: (1) using a thermostable FAS from $C$. thermophilum did not translate into structural stability of the complex as the structure was still limited to $3.2 \AA$. (2) The inability to visualize the ACP domains in the structural data most likely adds to structural variability in the complex. In the Saccharomyces cerevisiae FAS (ScFAS), the ACP domains tend to be docked at the ketosynthase active site. ScFAS, with its stalled ACP domains, might offer a more stable protein structure for achieving resolutions below $3 \AA$. The ability to visualize the ACP domain would also allow me to study the effects of ACP domain movement on the FAS structure. 


\subsection{Saccharomyces cerevisiae FAS as a model system}

\subsubsection{Purification of endogenous FAS from Saccharomyces cerevisiae}

The protease-deficient yeast strain BJ2168 (MATa prc1-407 prb1-1122 pep4-3 leu2 trp1 ura352 gal2) was used to obtain higher intracellular concentrations of FAS as well as to reduce proteolytic cleavage of the protein during purification. The conditions used for purifying ScFAS were distinct from those used for CtFAS due to differences in precipitation behavior of the FAS from the two species (Figure 10 (i)). The ScFAS precipitated from the S100 yeast lysate between 20-30\% PEG400 concentration. The co-precipitated impurities were then separated in three subsequent steps involving sucrose gradients followed by precipitation using $40 \%$ PEG400. With this protocol $15-20 \mathrm{mg}$ of protein were routinely obtained from $\sim 233 \mathrm{~g}$ of cells (wet weight). Interestingly, unlike the CtFAS purification, purified ScFAS was devoid of protein complexes such as the oxoglutarate dehydrogenase. However, there was a prominent $20 \mathrm{kDa}$ protein (as per SDS-PAGE) that was found to co-purify with the ScFAS (Figure 10 (ii)). A protein this small would normally sediment in the top fractions of gradients used for FAS purification. The co-sedimentation of this $20 \mathrm{kDa}$ protein with the FAS in the $40 \mathrm{~S}$ region was, therefore, surprising. This can only be possible either by a direct interaction of this protein with the FAS, or if it assembles into a large multimeric complex with a sedimentation coefficient of $\sim 40$ S. Both scenarios have never been reported and I therefore asked two questions - what is the identity of this small protein? Why is it found in the $40 \mathrm{~S}$ region of the sucrose gradients? 

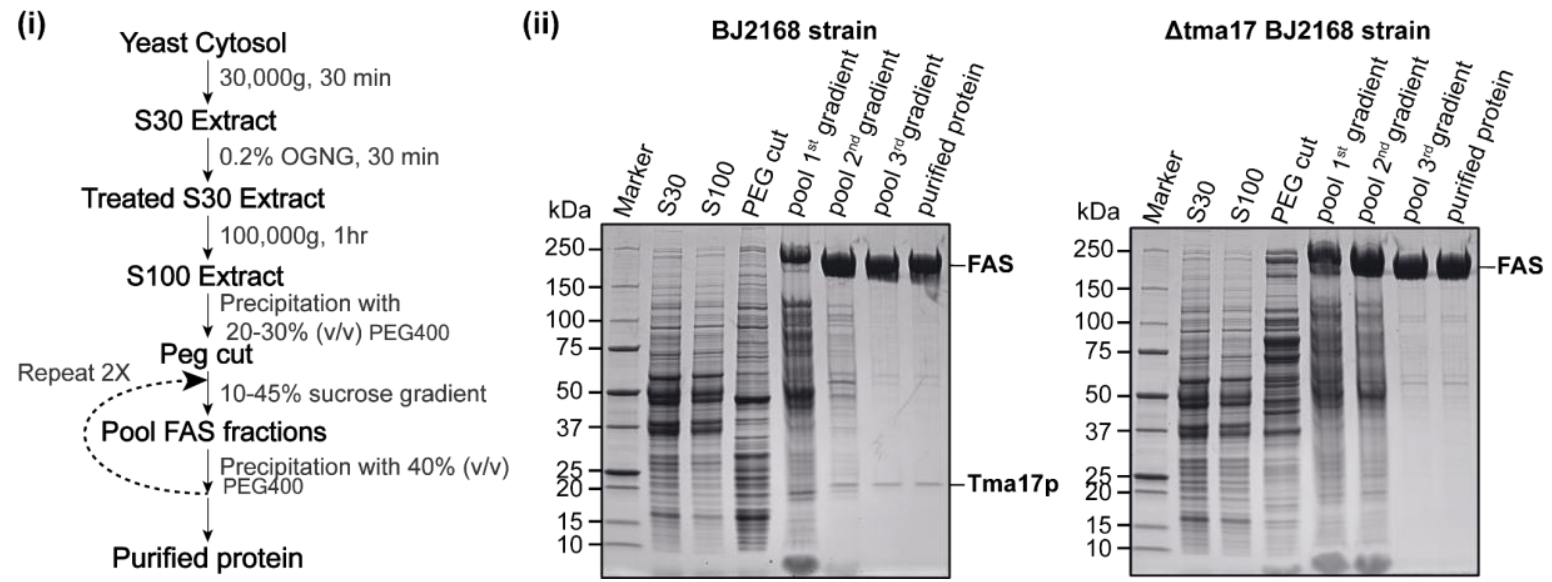

Figure 10. Purification of S. cerevisiae FAS (i) Schematic representation of the chromatographyfree purification established for S. cerevisiae FAS. The chromatography purification of ScFAS involved specific precipitation of FAS using PEG followed by sucrose density gradients. (ii) SDSPAGE analysis of FAS purification from BJ2168 S. cerevisiae strain. Aliquots of S30 (lane 2) and S100 (lane 3) extracts, the resuspended PEG cut (lane 4), the pool of the first, second and third sucrose gradients (lanes 5, 6 and 7) as well as the final purified protein preparation (lane 7) and a molecular weight marker (lane 1) are depicted. Note the distinct co-purification of a protein with an apparent molecular weight of $20 \mathrm{kDa}$. (iii) SDS-PAGE analysis of FAS purification from a $\triangle$ tma17 S. cerevisiae strain. Aliquots of the same fractions as in (ii) are loaded. Note the absence of the protein with an apparent molecular weight of $20 \mathrm{kDa}$.

\subsubsection{Discovery of a novel interactor of the yeast FAS}

The 20kDa band on the SDS-PAGE gel was identified by tandem mass spectrometry to be a protein previously reported to be either associated with ribosomes as Tma17p (Fleischer et al., 2006) or to represent a stress induced chaperone for $26 S$ proteasome assembly as Adc17p (Hanssum et al., 2014; Rousseau and Bertolotti, 2016). It is mainly found in fungi (Hanssum et al., 2014) (Figure 11). Three segments (1-53, 66-110 and 146-150) of Tma17p are conserved among the different species, whereas the remaining part of the protein appears to have diverged over the course of evolution. Since both negative stain EM and tandem mass spectrometry did not detect ribosome or $26 \mathrm{~S}$ proteasome contamination (Supplementary Table 1), I further investigated Tma17p. For this, a Tma17p deletion strain was generated and was used to purify the FAS using the same protocol as described above. Corroborating the results from mass spectrometry, the 20kDa band was absent from the proteins purified from the $\Delta$ tma17 strain (Figure 10 (ii)), thus, confirming that this small copurifying protein is indeed Tma17p/Adc17p. 


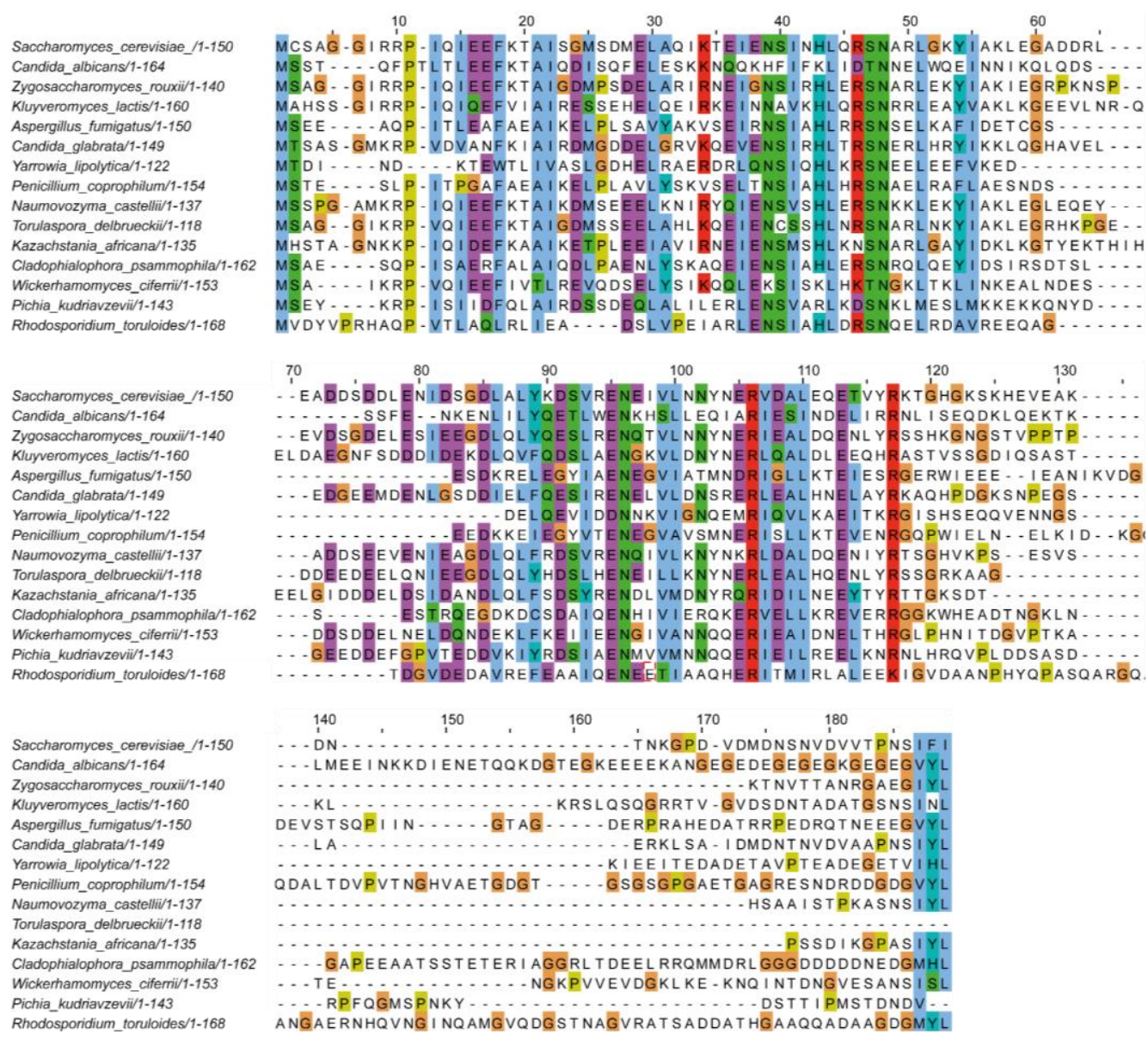

Figure 11. Tma17p is conserved in fungi. Sequences from Tma17p homologues from different fungal species were obtained from the Uniprot database and multiple sequence alignment was performed using the T-Coffee package (Notredame et al., 2000). The residues are colored according to the ClustalX scheme, where the conserved residues are labelled as follows: blue (hydrophobic), red (positively charged), magenta (negatively charged), green (polar), orange (glycine), yellow (proline) and cyan (aromatic).

Is Tma17p an independent multimeric $40 \mathrm{~S}$ complex or is it associated with the FAS? To answer this question, I visualized the FAS with the co-purified Tma17p using negative stain electron microscopy. The micrographs contained particles with dimensions and appearance typical of the FAS with no indications of any other complex being present in the sample. Further, the recombinant Tma17p expressed in E. coli did not sediment in the 40S region of the sucrose gradients but had a sedimentation profile similar to what one would expect for $17 \mathrm{kDa}$ protein (Figure 12 (i)). This was distinctly different from what I observed for Tma17p purified along with FAS. These two observations suggested that Tma17p might be directly interacting with FAS. To test this hypothesis, I reconstituted the Tma17p-FAS complex in 
vitro. The binding assay performed involved titrating increasing concentrations of rhodamine-labelled Tma17p against a constant amount of $\Delta$ Tma17p-FAS. The bound Tma17p was then separated from the unbound fraction using a native agarose gel (Figure 12 (ii)). The FAS migrated slower than the labelled Tma17p owing to the large difference in their molecular weights and charge distribution. The fluorescence signal from the region corresponding to the FAS was then measured to detect the bound Tma17p (Figure 12 (ii)). The fraction of labelled Tma17p bound to the FAS increases proportionally to the amount of labelled protein and displayed a sigmoidal behavior. Such a sigmoidal curve is characteristic of co-operative binding and therefore the Hill equation was used for fitting the data points. The binding of Tma17p to FAS has a $\mathrm{K}_{0.5}=2.1 \pm 0.2 \mu \mathrm{M}$ suggesting a moderate affinity. Along with this, the binding curve had a Hill coefficient $\left(\mathrm{n}_{\mathrm{H}}\right)$ of $2.1 \pm 0.3$. A Hill coefficient of 2 indicates a positive binding cooperativity, where more than one Tma17p molecule binds per molecule of FAS and the binding of one Tma17p enhances the binding of other Tma17p molecules. Another valuable information from this binding curve was that the concentration of Tma17p required to fully saturate the FAS was $\sim 6 \mu \mathrm{M}$. Therefore, in all future structural and biochemical experiments, Tma17p concentrations in excess of $6 \mu \mathrm{M}$ were used to reconstitute the Tma17p-FAS complex. 
(i)

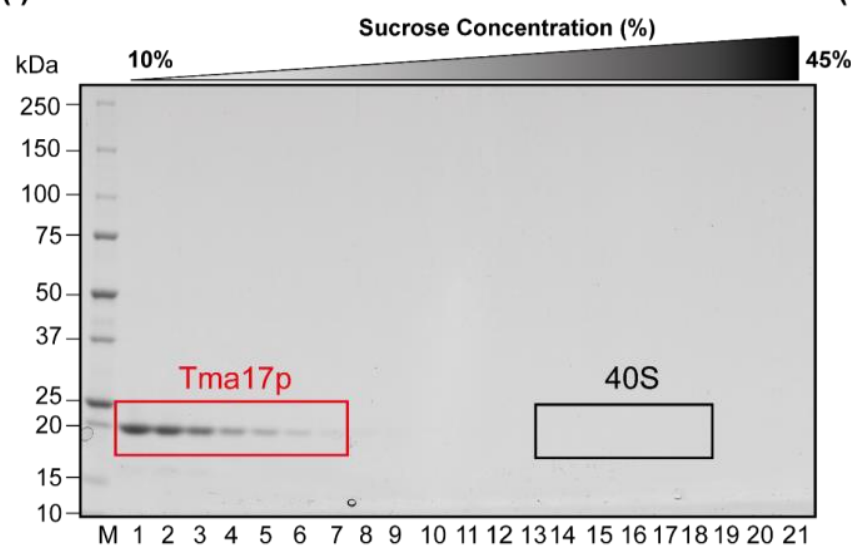

(ii)
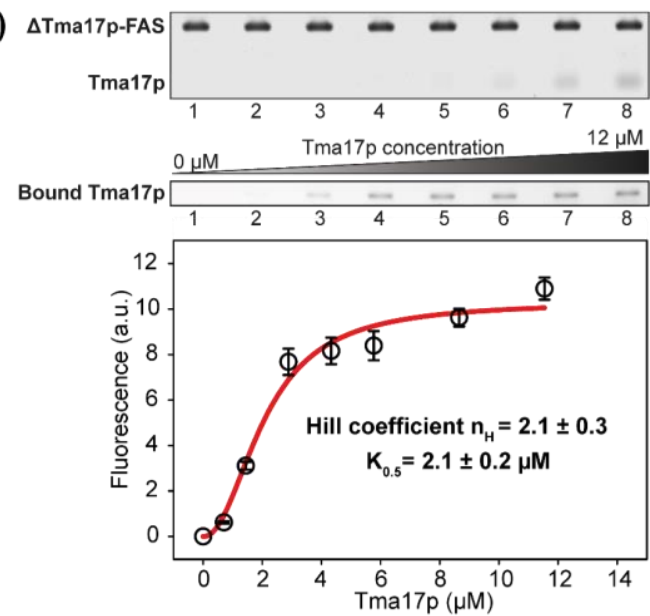

Figure 12. Tma17p binds to the FAS. (i) SDS-PAGE analysis of sucrose density gradient centrifugation of isolated, recombinant Tma17p in gradients utilized to resolve FAS. The recombinant Tma17p protein is present in the top fractions of the gradient (red) and its sedimentation behavior is distinct from when it is bound to FAS (black). (ii) Reconstitution of the Tma17p-FAS complex. The top left depicts a Coomassie-stained native gel, where the positions of migration of FAS and Tma17p are indicated. The middle panel shows a fluorescence detection of the FAS region of the gel to visualize FAS-bound Tma17p, the bottom panel represents the quantitative analysis of the fluorescence signal. Hill equation was used for fitting the data points.

Finally, Tma17p is also known to be phosphorylated in vivo (Albuquerque et al., 2008; Holt et al., 2009) and the endogenous Tma17p purified with the FAS was indeed found to be phosphorylated using mass spectrometry. To check for the impact of such modifications on Tma17p's affinity to the FAS, I compared the stability of endogenously purified Tma17pFAS complex versus the reconstituted one. To study this, the stability of the Tma17p-FAS complex with respect to increasing salt concentrations was used as an indicator for Tma17p's affinity to the FAS. For this, the respective samples were loaded onto sucrose gradients containing $50 \mathrm{mM}, 150 \mathrm{mM}$ and $250 \mathrm{mM} \mathrm{KCl}$. For the reconstituted complex, Tma17p completely dissociated at $150 \mathrm{mM}$ salt concentration. In comparison, the endogenous complex was more stable, with approximately half of the bound Tma17p dissociating at $150 \mathrm{mM} \mathrm{KCl}$ and a complete dissociation occurring at $250 \mathrm{mM} \mathrm{KCl}$ (Figure 13). This suggested that the interaction of endogenous Tma17p with the FAS is stronger than that of the recombinant version. Along with this, the complete dissociation of Tma17p from FAS at $250 \mathrm{mM} \mathrm{KCl}$ suggests that Tma17p-FAS interaction could have escaped identification due to the dissociation of Tma17p from the FAS because of the relatively high- 
salt conditions (between 0.2-1M) previously employed to purify the FAS (Leibundgut et al., 2007; Lomakin et al., 2007).

(i)

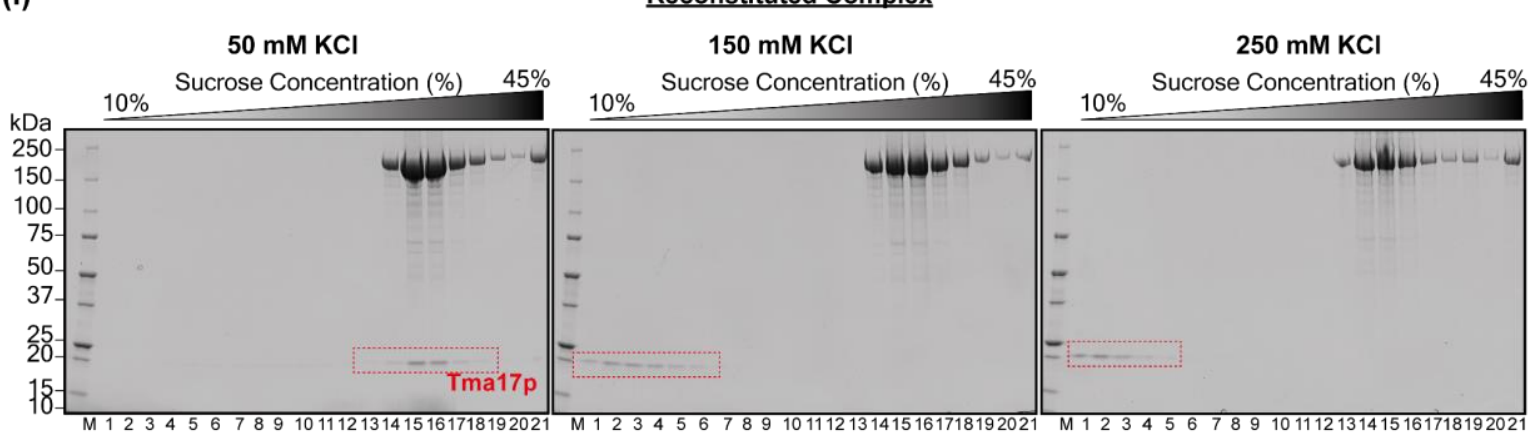

(ii)

Endogenous Complex

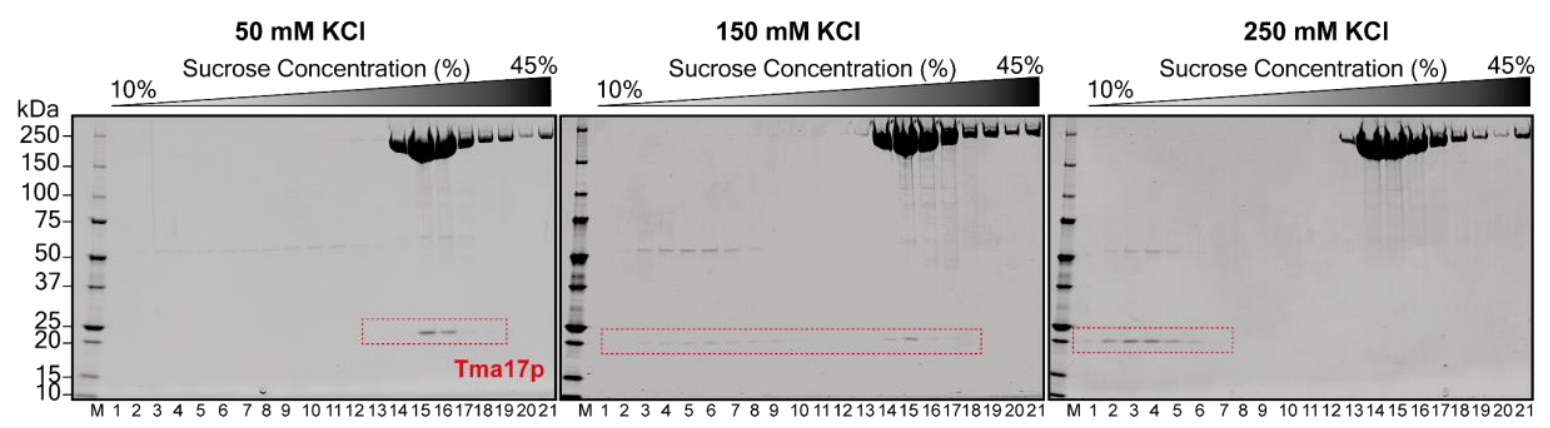

Figure 13. Dissociation of endogenous Tma17p from the FAS is less salt-labile than the recombinant Tma17p. SDS-PAGE analysis of sucrose density gradient centrifugation of (i)

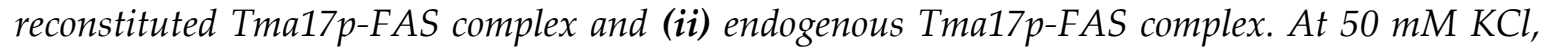
Tma17p is stably associated with FAS and co-sediments in the $40 S$ region of the gradient (lanes 1417). In contrast, gradient centrifugation at $150 \mathrm{mM} \mathrm{KCl}$ causes the partial dissociation of endogenous Tma17p (lanes 2-9 and 14-17) and complete dissociation of recombinant FAS (lanes 17). At $250 \mathrm{mM} \mathrm{KCl}$, endogenous Tma17p is completely dissociated from FAS and sediments in the top fractions of the gradient (lanes 1-7).

\subsubsection{Mass spectrometric analysis of Tma17p and Tma17p-FAS complex}

All the following mass spectrometric data analysis was performed in collaboration with Andreas Linden and Henning Urlaub (Bioanalytical Mass Spectrometry group, Max Planck Institute for Biophysical Chemistry). 
Data from proteome wide screens showed that Tma17p can be phosphorylated at serine 24, 68 and 76 (Albuquerque et al., 2008; Holt et al., 2009). We therefore used mass spectrometry to determine whether the endogenous Tma17p purified with the FAS carries a phosphorylation as well as to determine the identity of the phosphorylated residues. We found several phosphorylated residues on both the FAS and Tma17p. Interestingly, along with all serine residues in the Tma17p reported previously to be modified, serine 38 was also found to be phosphorylated. The significance of the post-translation modifications on Tma17p were not investigated further during this thesis as I primarily focused on characterizing the effect of Tma17p on FAS activity and structure.

Furthermore, crosslinking mass spectrometry (XL-MS) was used to gain more insight into the interaction between the Tma17p and FAS. Considering the abundance of aspartate and lysine residues in both, Tma17p and FAS, crosslinkers were chosen such that they bind to either of these two residues. Three independent trials using SDA (succinimidyl 4,4'azipentanoate), EDC (1-ethyl-3-(3-dimethylaminopropyl)carbodiimide hydrochloride) and BS3 (bis(sulfosuccinimidyl) suberate) crosslinkers were performed. EDC and SDA are zerolength crosslinkers which couple short range interactions $(<5 \AA)$. EDC links primary amines (such as Lys) with closely located carboxyl groups (such as Asp and Glu), whereas SDA links primary amines to any amino acid side chain in its proximity. BS3 is an amine-toamine crosslinker that can link residues 10-20 Å apart. The mass spectra obtained from this analysis indicated that all the FAS residues in the vicinity of the Tma17p lie inside the barrel of the complex (Figure 14, Supplementary Table 2). Residues 55-72 of the Tma17p seem to have short range interactions near the active site cleft of the MPT domain, whereas residues 124 and 129 are near the KS domain. Along with this, all three crosslinkers suggested that the Tma17p also interacts with the ER domain of FAS. The proximity of a $17 \mathrm{kDa}$ protein to the MPT, ER and KS domain hinted at two possibilities: (1) Tma17p has multiple binding sites inside the dome of FAS or, (2) it is structured in a way that it simultaneously interacts with all the domains over a distance of $\sim 120 \AA$. 


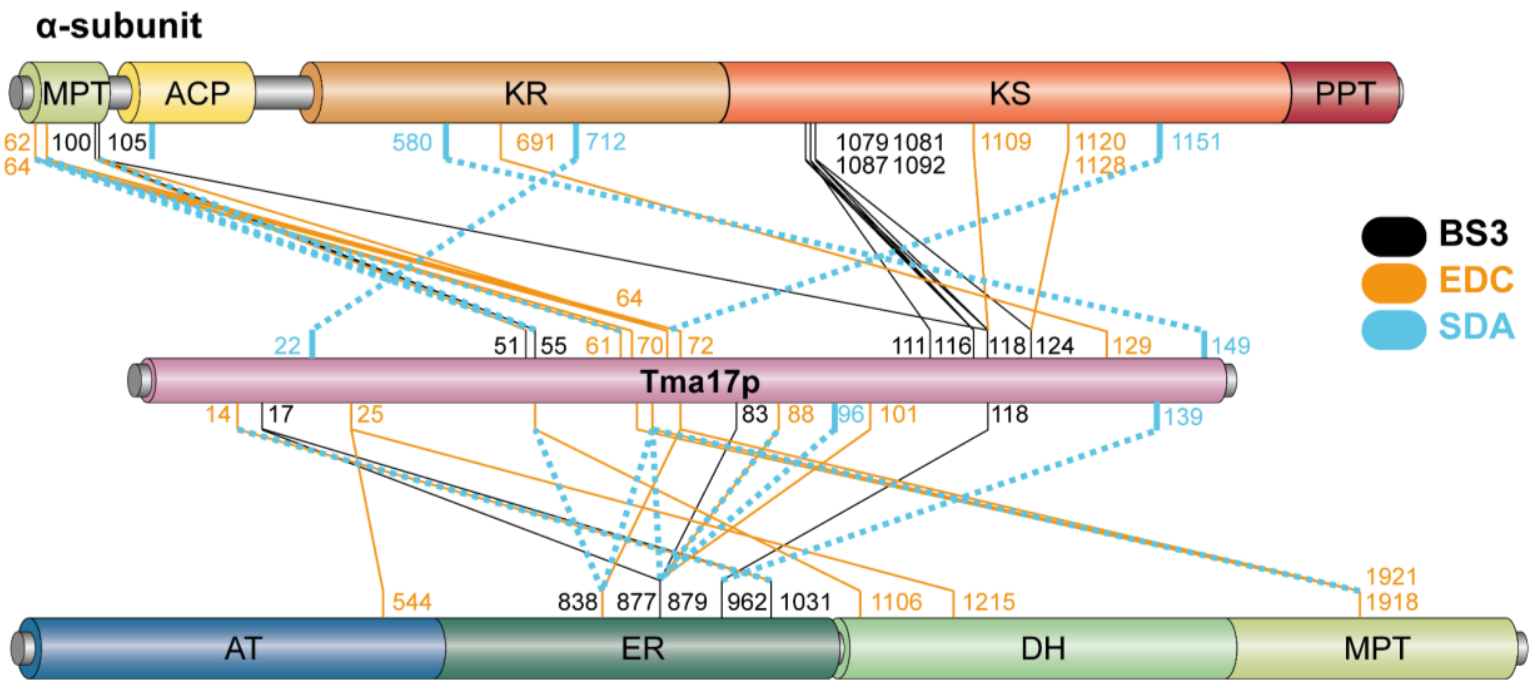

\section{$\beta$-subunit}

Figure 14. XL-MS analysis of the Tma17p-FAS complex. Crosslinks between Tma17p and FAS using BS3 (black), EDC (orange) and SDA (cyan) crosslinker are depicted. The $N$-terminal residues of Tma17p are located near the ER domain, the central residues are near the ER and MPT domains and the C-terminal residues are in proximity of the KS domain.

\subsubsection{Effect of FAS activity upon binding of Tma17p}

XL-MS data suggested that the Tma17p interacts within the interior of the FAS dome and is in proximity of active site clefts of at least the MPT and ER domains. To examine if such interactions affect the activity of the FAS, I collaborated with Benjamin Graf (Department of Structural Dynamics, Max Planck Institute for Biophysical Chemistry) to determine steady state kinetics. The data was analyzed by Victor Sautner and Kai Tittmann (Department of Molecular Enzymology, Georg August University Goettingen).

To determine steady state kinetics of the FAS, the initial rate of change in NADPH absorbance was measured. NADPH absorbs light at a wavelength of $340 \mathrm{~nm}$ whereas its reduced form, NADP+ does not. Since for each fatty acid synthesized, FAS uses 1 acetylCoA, 7 malonyl-CoA and 14 NADPH molecules, measuring the rate of change in NADPH reduction correlates to FAS activity. FAS activity was then measured in the presence and absence of saturating concentrations $(12 \mu \mathrm{M})$ of Tma17p. The concentration of each substrate (acetyl-CoA, malonyl-CoA and NADPH) was titrated individually, while keeping the other two at saturating levels. Three curves were obtained in the end, one for each 
substrate. These kinetic measurements allowed for the detection and identification of the effects of Tma17p binding on FAS activity.

The steady state kinetic measurements revealed saturation kinetics along with co-operative binding for acetyl-CoA and malonyl-CoA with an apparent binding affinity (Км $\left.{ }^{\text {app }}\right)$ of $6.5 \pm$ $0.5 \mu \mathrm{M}$ and $6.5 \pm 0.7 \mu \mathrm{M}$, respectively (Figure 15(ii,iii)). The data for NADPH, however, did not exhibit similar behavior to the other substrates and could be best explained by a substrate dependent activation model. This model has two phases, the first phase follows a hyperbolic behavior where the $\mathrm{K}_{\mathrm{m}}^{\text {app }}$ for $\mathrm{NADPH}$ was $9.1 \pm 1.8 \mu \mathrm{M}$, whereas the second phase has a linear, non-saturating behavior. The second phase is indicative of activation of the enzyme at higher substrate concentrations and has an activation constant (Kact) of $3.0 \pm$ $0.5 \mathrm{mM}$ (Figure 15(i)). In comparison, for the Tma17p-FAS complex, even though the general behavior of acetyl-CoA and malonyl-CoA turnover rates were similar to that of the FAS where the $\mathrm{KM}^{\text {app }}$ increased to $12.2 \pm 1.8 \mu \mathrm{M}$ and $22.4 \pm 2.4 \mu \mathrm{M}$, respectively. For NADPH, the enzyme complex exhibited a classic, hyperbolic Michaelis-Menten dependence with a KM of $155 \pm 29 \mu \mathrm{M}$.

The kinetic analysis revealed that the FAS activity is affected upon binding of Tma17p. In the presence of Tma17p, the affinity of the FAS for its substrates - acetyl-CoA, malonylCoA and NADPH - decreased by 2-, 4- and 16-fold, respectively. This novel FAS binding protein appears to have an inhibitory activity, where the mode of action varies from a noncompetitive one for acetyl-CoA to a competitive one for malonyl-CoA. In the case of $\mathrm{NADPH}$, there was a complete change in the response of FAS towards increasing NADPH concentrations. This was evident by the change from a substrate-dependent activation model in the absence of Tma17p to a classical hyperbolic behavior in the presence of Tma17p.

All the biochemical evidence presented until now indicate that Tma17p binds the FAS in a specific manner. It also has the ability to affect FAS activity. Based on these observations, Tma17p will now be referred to as an accessory " $\gamma$-subunit" of the FAS, the Tma17p-FAS complex as FAS holoenzyme and FAS without the Tma17p as FAS. 

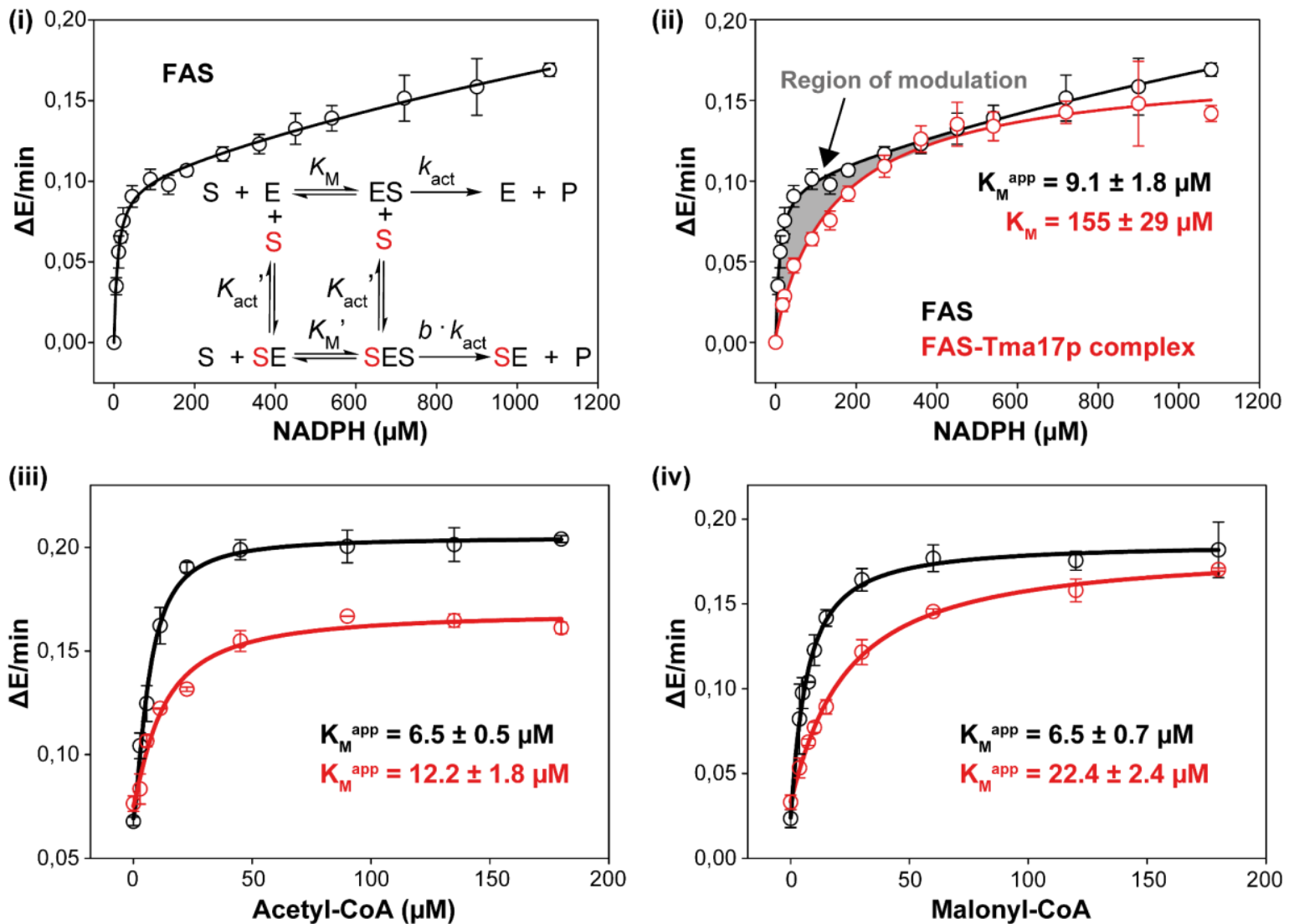

Figure 15. Steady-state kinetic analysis of FAS in the absence and presence of Tma17p. Substrate concentration dependence of FAS and Tma17p-FAS for substrates: NADPH (i)(ii), acetylCoA (iii) and malonyl-CoA (iv). The plots depict initial velocities of FAS (black) and Tma17p-FAS (red) versus the concentrations of the respective substrate. The substrate activation kinetic model used to describe the NADPH dependence of FAS is shown as an inset in (i).

\subsubsection{Molecular basis underlying the $\gamma$-subunit's inhibitory activity}

To further understand the molecular basis underlying the $\gamma$-subunit's ability to bind and inhibit the FAS, I studied the effect of the $\gamma$-subunit on the FAS structure. To this end, I performed structural and conformational landscape analysis of FAS as well as the FAS holoenzyme complex. Since, the $\gamma$-subunit can partially dissociate from the FAS during purification and cryo-EM sample preparation, FAS holoenzyme reconstituted by addition of saturating amounts of the $\gamma$-subunit to the FAS was also used in addition to the endogenous complex for structure elucidation. Along with this, due to the differences between previously reported ScFAS structures solved by cryo-EM and X-ray crystallography (Gipson et al., 2010; Jenni et al., 2007; Leibundgut et al., 2007), I used both 
the techniques in parallel to cross-validate the structural findings and avoid misinterpretation of the data.

\subsubsection{Cryo-EM analysis of endogenous FAS holoenzyme complex}

For the endogenous FAS holoenzyme complex 14,968 micrographs were acquired on a Titan Krios with a Cs corrector at $300 \mathrm{kV}$ using a Falcon III direct electron detector in integrating mode (Supplementary Table 3). Good contrast and particle density aided selection of 1,305,760 particles which were classified in 2D to sort out empty images or those displaying only low-resolution features (Figure 16). This reduced the dataset by $28 \%$. It is worth drawing the reader's attention here again to the fact that the central wheel composed of the $\alpha$-subunit - is very densely packed and is the most stable part of the complex. In contrast, the $\beta$-subunit forms the two dome-like structures where the interdomain regions have numerous exposed surfaces allowing for conformational variability. The most successful strategy for sorting the different FAS conformations was based on focused 3D refinement using D3 symmetry on the central wheel to obtain the alignment parameters for the particle images. These alignment parameters were then used for sorting the different 3D conformations while focusing on the conformationally variable dome region. Using the whole molecule or focusing on the FAS dome for 3D refinement was found not to be as effective for sorting conformations. Taking this into account, the remaining 789,813 particles were then iteratively used for multiple rounds of focused refinement, 3D classification and 2D classification using Relion 3.0 (Zivanov et al., 2018) to select for the most stable conformation of FAS which can then be resolved at high resolution (Figure 16). After the first round of 3D classification, two states of the FAS were observed, one with $40.6 \%$ of the particles with a conformation identical to the published FAS structures, whereas, $59.4 \%$ of particles had a different "rotated" conformation of the dome. The rotation of the dome was due to a large shift in the AT and MPT domains (Figure 17(i)). In this rotated conformation, the density corresponding to the ACP domain was localized next to the AT active site. Further, iterative rounds of focused 3D refinement and 3D classification were performed separately on the two conformational states. During each sorting step, the major difference between different 3D classes was the resolution of the 
resulting 3D volumes instead of conformational differences. Only the particles belonging to the best resolved class were used for the subsequent round of sorting. This approach resulted in a $3.2 \AA$ structure of the rotated conformation using 127,305 particles and a $3.3 \AA$ structure of the non-rotated conformation using 81,635 particles, estimated using the FSC 0.143 criterion. Both 3D maps displayed local resolution variations in the range of $3-4 \AA$.

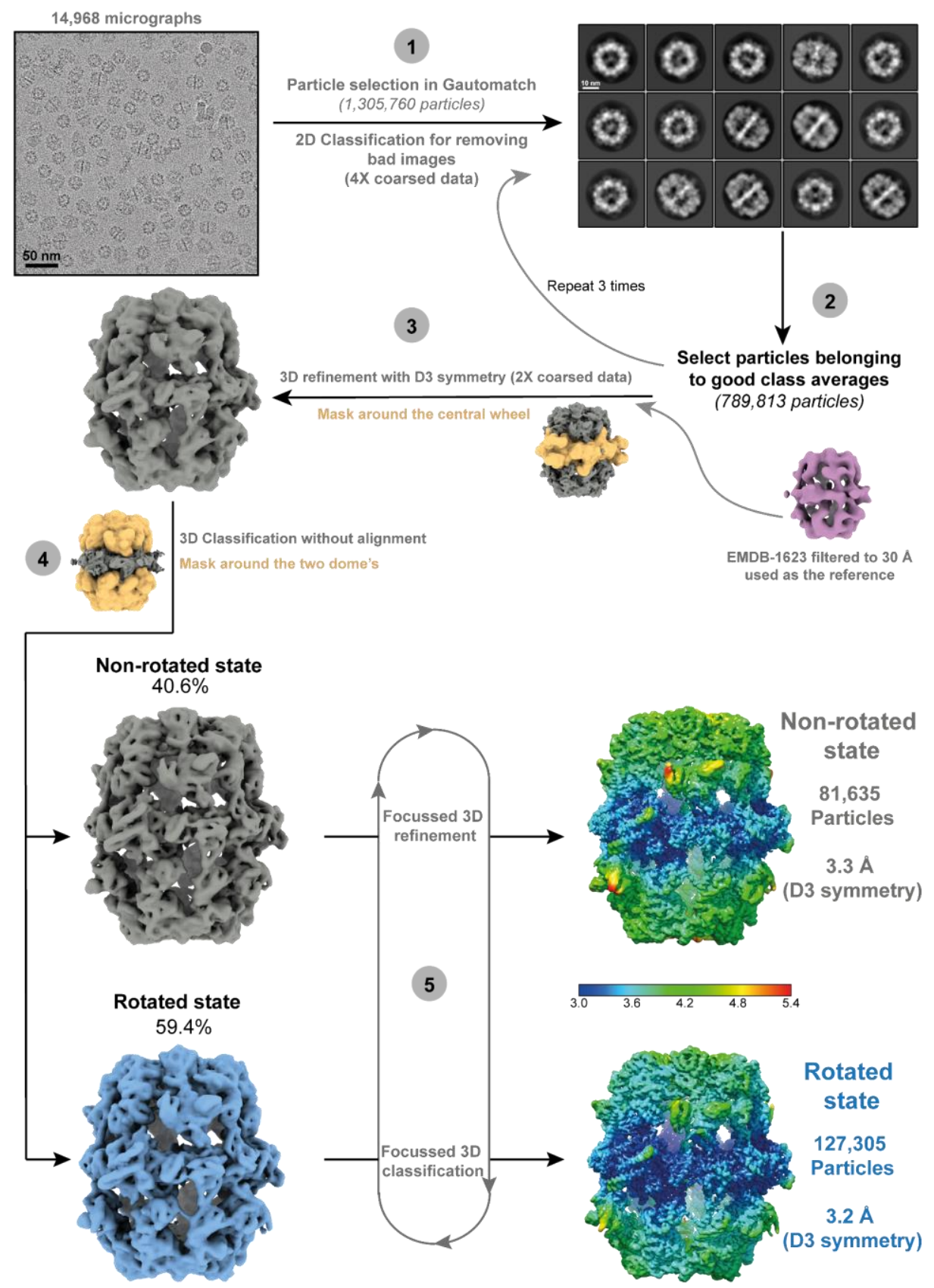

Figure 16. Scheme for cryo-EM data processing for endogenous FAS holoenzyme. The scheme depicts the different image processing steps performed. (1) Motion-corrected and dose weighted micrographs were used for particle selection. (2) Selected, individual particles were classified in $2 D$ 
for 3 rounds, where particles belonging to class averages with bad optical properties or low signalto-noise were removed after each round. (3) The selected particles were then used for $3 D$ refinement using D3 symmetry with the published cryo-EM structure of S. cerevisiae FAS (EMDB - 1623) as a reference. During the 3D refinement, a mask was placed around the central wheel to focus the refinement around the most stable part of the protein complex. (4) The alignment parameters of the particles from the previous step were used for classifying the particles in 3D. For 3D classification, a mask around the dome of the FAS was used to sort the major movements of this molecule, the rotated and the non-rotated conformations. (5) Particles from the best 3D classes were selected followed by multiple rounds of $3 D$ refinement and classification as described above. Particles belonging to the class with the most uniform resolution were selected for a final $3 D$ refinement. The final 3D maps obtained had an overall resolution, estimated using the FSC 0.143 criterion, of $3.3 \AA$ for the non-rotated state and $3.2 \AA$ for the rotated state.

\section{Structural differences between the rotated and non-rotated state of the endogenous FAS holoenzyme complex}

The 3.1 Å FAS crystal structure (Leibundgut et al., 2007) (PDB 2UV8) correlated well ( 79\%) with the non-rotated conformation of the FAS except for two differences - (1) the ACP domain was not resolved in the cryo-EM map of the non-rotated state and (2) minor deviations in segments of the MPT domain. The unresolved ACP domain in the non-rotated conformation of the FAS was a significant variation from the published ScFAS crystal structure (Figure 17 (ii)). One reason for this could be the heterogeneity in the molecules bound to the phosphopantetheine prosthetic group of the ACP. Therefore, to stabilize the $\mathrm{ACP}$, I added an additional step during the ScFAS purification, where I added malonylCoA and NADPH to the FAS sample in order to complete the synthesis of all bound intermediates. This was done to bring all the ACP domains of the FAS to the same functional state, where all the phosphopantetheine groups are empty. This process of adding FAS substrates to remove the ACP bound intermediates is here forth referred to as "FAS cycling". The FAS samples used for all subsequent experiments were "cycled" during purification to bring all the molecules to the same functional state.

The second, rotated conformation of the FAS, displayed large shifts of AT and MPT domain when compared to the crystallographic FAS structure (Leibundgut et al., 2007). The crystallographic model was fit into the rotated state map interactively in Coot (Emsley and Cowtan, 2004) and then refined using Refmac5 (Murshudov et al., 2011). Most of the 
conformational differences of the dome were explained by rigid body shifts of the different domains in the $\beta$-subunit. The AT domain shifts downwards by $7 \AA$ and the MPT domain moves outwards by $10 \AA$ (Figure 17(i)). The ER and DH domains, however, displayed only minor shifts of $\sim 4 \AA$ each and the $\alpha$-subunit was relatively unchanged. The overall length of the FAS was reduced by $10 \AA$ as a result of these structural rearrangements of the FAS dome. In the rotated state, the ACP domain was stabilized next to the AT active site channel as compared to the non-rotated conformation of the crystal structure, where it is situated at the KS domain. Two additional densities which could not be explained by any of the structured or flexible FAS residues were observed only in the rotated conformation of FAS (Figure 17(ii)). The first one was in the KR domain substrate binding cleft and the other corresponded to a coiled coil density, which was present next to the ER domain. Based on the XL-MS data, the location of the extra densities was in close proximity ( 10-30 $\AA$ ) to the residues in the ER, MPT and KS domain that formed crosslinks with the $\gamma$-subunit. Therefore, I hypothesized that these densities corresponded to the $\gamma$-subunit, which was characterized in the previous chapters to co-purify with, interact with and inhibit the FAS. 
(i)

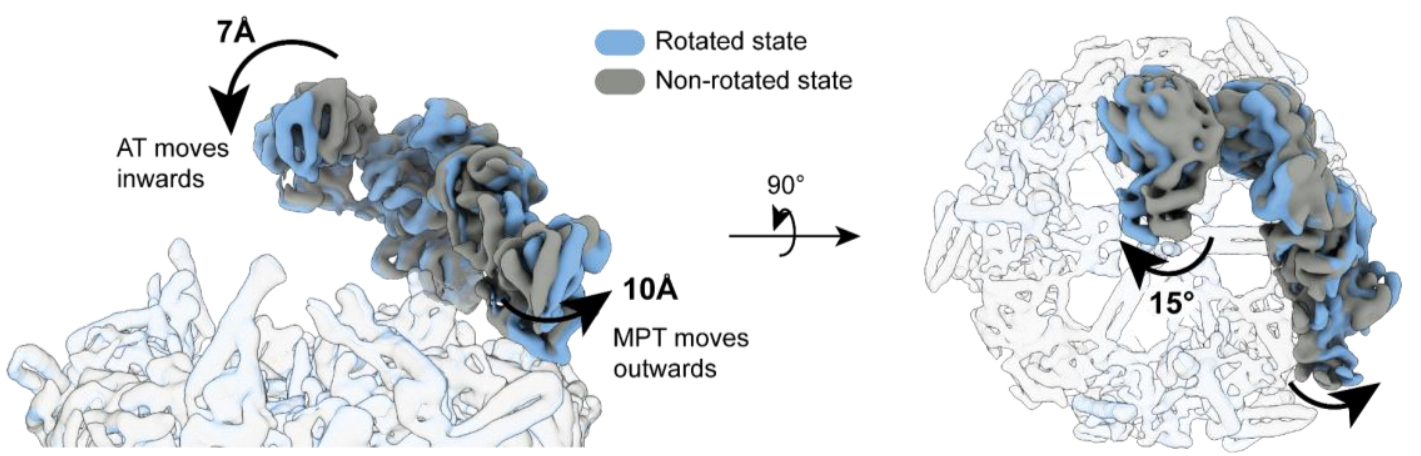

(ii)
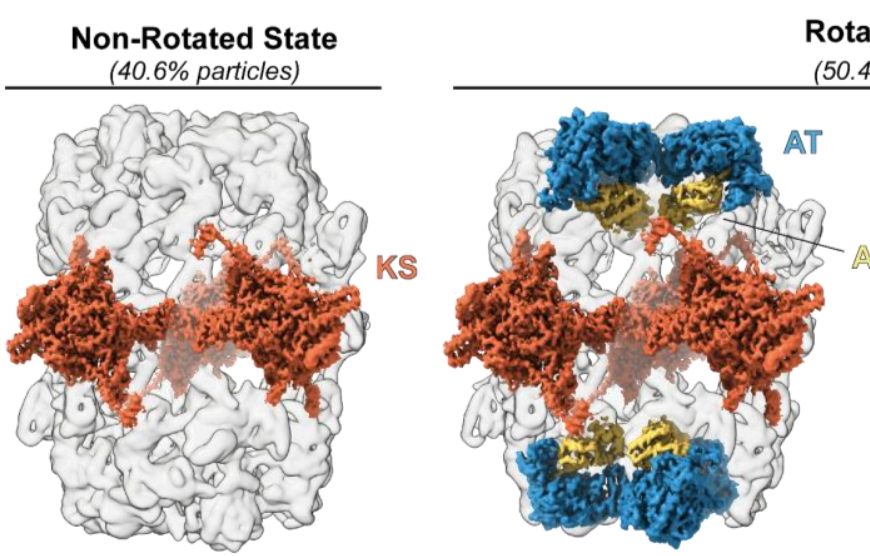

Rotated State

$50.4 \%$ particles)

Figure 17. Structural differences between the non-rotated and rotated conformations of the endogenous FAS holoenzyme complex. (i) Overlay of $\beta$-subunits of the non-rotated (grey) and rotated (blue) conformation of the FAS. In the rotated conformation of the FAS, each $\beta$-subunit is rotated by $15^{\circ}$ and the overall length of the molecule decreases by $10 \AA$. This results in an inward shift of the AT domain by $7 \AA$ and an outward movement of the MPT domain by $10 \AA$. (ii) In the left panel, the non-rotated conformation is shown, where no ACP domains are observed. Rotation of the $\beta$-subunit correlates with the stabilization of the ACP domain (yellow) at the AT domain (blue). Additionally, a coiled coil density was found directly adjacent to the ACP domains in the rotated state, as well as a small density fragment at the KR domains (pink).

Similar observations in terms of the FAS dome conformation were also reported by Gipson et al. (2010), in their $6 \AA$ A cryo-EM structure of cerulenin inhibited FAS. Their structure was similar to the rotated state presented here and also consists of a coiled coil density next to the ER domain. Gipson et al. (2010) did not report the presence of additional proteins bound to the FAS and hypothesized that the coiled coil density most likely belonged to the ACP linker arms which become structured in the rotated conformation of the FAS. Taking these previous observations into consideration, I proceeded with testing whether the FAS can adopt the rotated conformation in the absence of the $\gamma$-subunit to confirm the identity of these extra densities. Both X-ray crystallography and cryo-EM were used for structure 
determination of the FAS in the absence of the $\gamma$-subunit for cross-validation of structural findings.

\subsubsection{Cryo-EM structure determination of FAS in the absence of the $\gamma$-subunit}

For determining the structure of the FAS in the absence of the $\gamma$-subunit, 5,541 micrographs were acquired on a Titan Krios at $300 \mathrm{kV}$ using a Falcon III direct electron detector in integrating mode (Supplementary Table 3). From these micrographs, 1,189,206 particles were initially selected. The image processing pipeline employed was similar to the one used for endogenous FAS holoenzyme complex (Supplementary Figure S1). The 834,204 particles selected after 2D classifications were used for two rounds of focused refinement and 3D classification using Relion 3.0 (Zivanov et al., 2018) to select for the different stable conformations of the FAS. The first round of 3D classification resulted in two distinct classes, one comprising $8.2 \%$ of the particles in the rotated conformation and the other 91.8\% in the non-rotated. The rotated conformation had the ACP domain located at the AT domain whereas the non-rotated conformation had the ACP located at the KS domain (Figure 18).

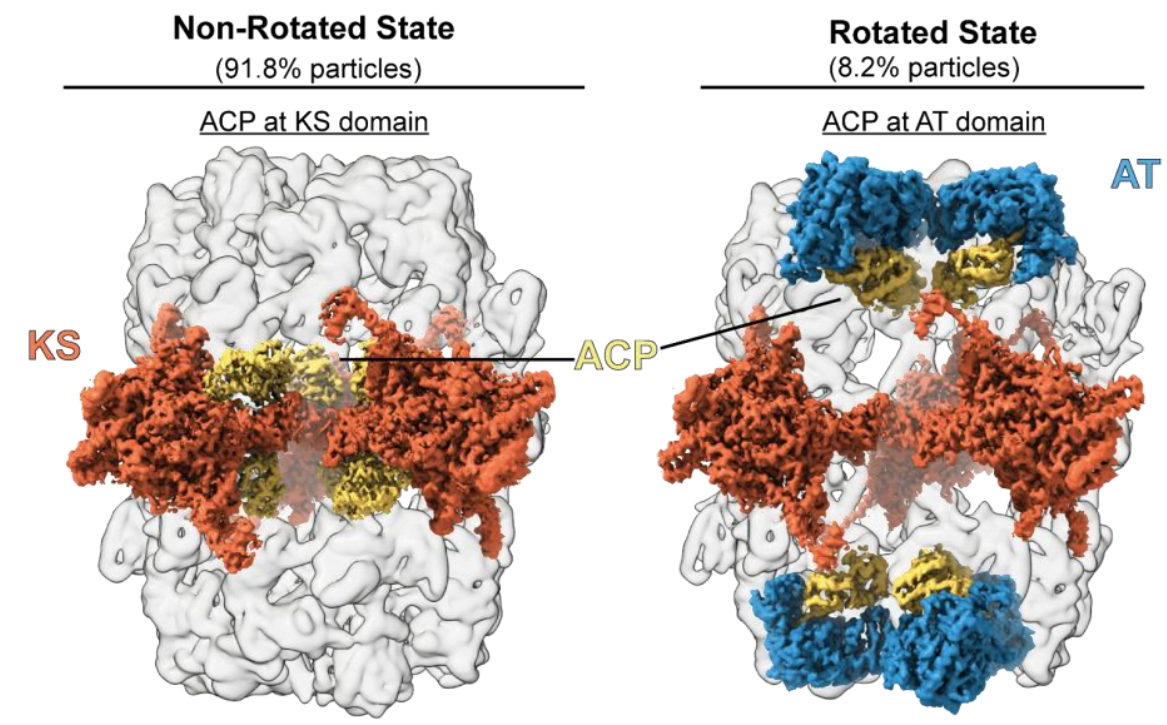

Figure 18. ACP location in the non-rotated and rotated conformations of the "cycled" FAS. In the left panel, the non-rotated conformation with the ACP domain at the KS active site is shown. In the right panel, the ACP located at the AT domain in the rotated FAS conformation is depicted. Notice that the ACP domain in the non-rotated state of the "cycled" FAS is resolved as compared to the non-rotated conformation of the non-cycled FAS (Figure 17). 
Particles belonging to the best class of the non-rotated FAS conformation then used for another round of sorting (Supplementary Figure S1). The data for the non-rotated state was further classified into 3 main classes which refined to $3.3 \AA$ (class 1 containing 20\% particles), $3.2 \AA$ (class 2 containing 25\% particles) and $3.1 \AA$ (class 3 containing $33 \%$ particles) resolution, estimated using the 0.143 FSC criterion. All the three 3D volumes were at comparable global resolutions and conformationally identical. However, significant local resolution differences in class 1 and 2 suggested that there was still some variability in the dome which resulted in poorly resolved features as compared to the central wheel. Therefore, particles belonging to class 3 were selected for further steps where the CTF parameters were refined in Relion 3.0. For the rotated conformation, all the particles after the first 3D classification were directly used for CTF refinement followed by 3D refinement. In the end, FAS structures of $2.9 \AA$ (using 144,526 particles) for the non-rotated conformation and $3.2 \AA$ (using 69,059 particles) for the rotated conformation were obtained with applied D3 symmetry (Figure 19(i)). The global resolutions were estimated using the FSC 0.143 criterion (Figure 19(ii)).

The crystallographic FAS structure (from the next section) was refined against the $2.9 \AA$ non-rotated FAS map (Supplementary Table 4). Densities corresponding to side chains were visible throughout the structure (Figure 19(iii)). The refined model had a CC of 0.9

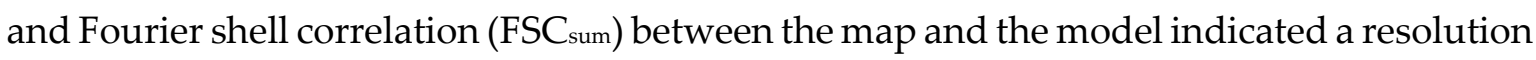
of $3 \AA$ as per the FSC 0.5 criterion (Figure 19(iv)). The refined FAS model was also validated by refining the crystallographic FAS structure against the first unfiltered half-map (FSC work$_{\text {) }}$. The refined structure from the first half-map was then compared with the second unfiltered

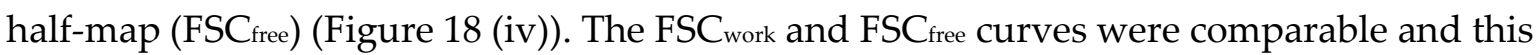
indicated that the refined models have not been over-fitted to the cryo-EM data. The rotated FAS map from this dataset was not used for model building as FAS structures with the same conformation were solved at higher resolution in the later sections of this thesis. Only the highest resolved rotated FAS structure was used for model building (section 3.2.5.4.). 
(i)

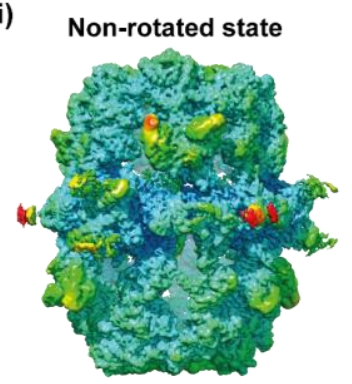

(iii)

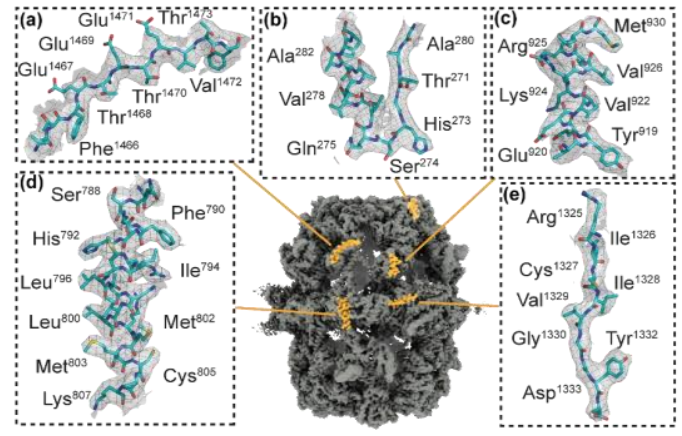

(ii)
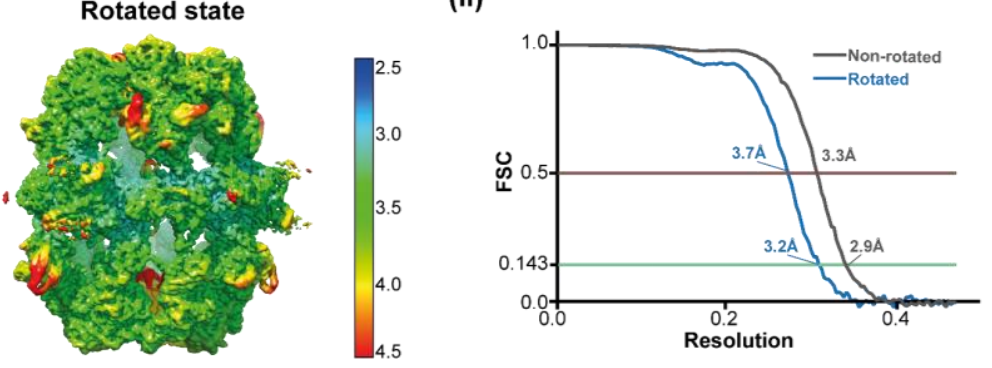

(iv)

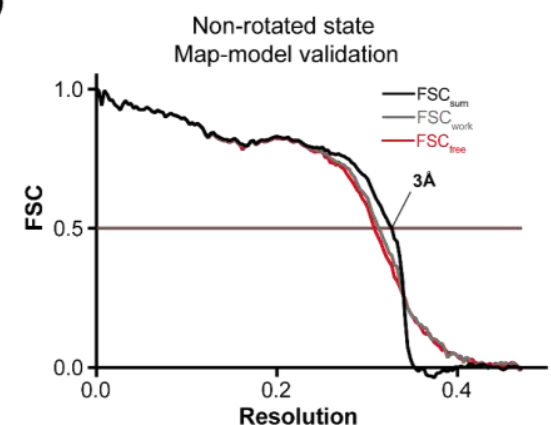

Figure 19. Cryo-EM structure of the FAS in the absence of the $\gamma$-subunit. (i) Local resolution maps of the non-rotated (left) and rotated (right) conformations of the FAS. The resolution range depicted is from $2.5 \AA$ (blue) to $4.5 \AA$ (red). The local resolution varies from $2.5 \AA$ to $3.5 \AA$ for the non-rotated state and from $3 \AA$ to $4 \AA$ for the rotated state. (ii) Fourier shell correlation (FSC) of two independently refined subsets of particles for the non-rotated (grey) and rotated (blue) conformation of FAS. The FSC curve indicates a resolution of $2.9 \AA$ for the non-rotated conformation and $3.2 \AA$ for the rotated conformation at a threshold of 0.143. (iii) Several excerpts within (a) DH, (b) $A T,(c)$ $E R,(d) K R$ and (e) KS domains displayed in cyan are utilized to show the quality of the final $2.9 \AA$ density map. (iv) Fourier shell correlation (FSC) curves of the final refined map of the non-rotated conformation and its model (FSC $C_{\text {sum }}$ ), the first unfiltered half-map and its model (FSC work) as well as the second unfiltered half-map and the model from the first unfiltered half-map (FSC free). The FSC $C_{\text {sum }}$ curve indicates a resolution of $3 \AA$ at a threshold of 0.143. The FSC work and FSC free overlap with each other and indicate that the model is not affected by overfitting.

\subsubsection{Crystallographic structure determination of FAS in the absence of the $\gamma$ -}

\section{subunit}

The FAS sample used for growing crystals was prepared similarly to the one used for cryoEM sample preparation. I crystallized the FAS using the sitting drop vapor diffusion technique with the following buffer: 0.3 M sodium malonate, $11.5-13 \%$ (w/v) PEG3350 and 0.1 M HEPES pH 7. The crystals diffracted X-rays to $2.9 \AA$ and belonged to the primitive monoclinic space group P2 1 with unit cell constants of $a=217.6 \AA, b=347.6 \AA, c=265.3 \AA$, 
$\beta=107.9^{\circ}$ with a single FAS molecule in the asymmetric unit. The structure was solved by molecular replacement using the previously reported $3.1 \AA$ S. cerevisiae structure (Leibundgut et al., 2007). The resulting electron density displayed features corresponding to side chains throughout the whole molecule including the PPT domain, which was absent in the model used for phasing (Figure 20(i)). The PPT domain was thus modelled into the density and was found to have a similar structure to that solved for an isolated PPT domain (Johansson et al., 2009) (Figure 20(ii)). The only sequences that could not be modelled in this structure were the same flexible loop segments which have always been unresolved in previous reported structures. The model after refinement had average B-factors of $92 \AA^{2}$ and $\mathrm{R} / \mathrm{R}_{\text {free }}$ of $19.7 \%$ / $21.8 \%$. The diffraction data was also analyzed using the STARANISO server, where a mild anisotropy in diffraction of $\sim 0.1-0.2 \AA$ was detected (Supplementary Figure S2). Anisotropically truncated data, taking into account diffraction spots up to $2.8 \AA$ in certain directions, was then used for model refinement. The side chain and solvent densities improved resulting in an improved model with average B-factors reduced to $\sim 73$ $\AA^{2}$ and R/R free $_{\text {of }} 19.2 \%$ / 21.1\% (Supplementary Figure S2, Supplementary Table 4).

(i)

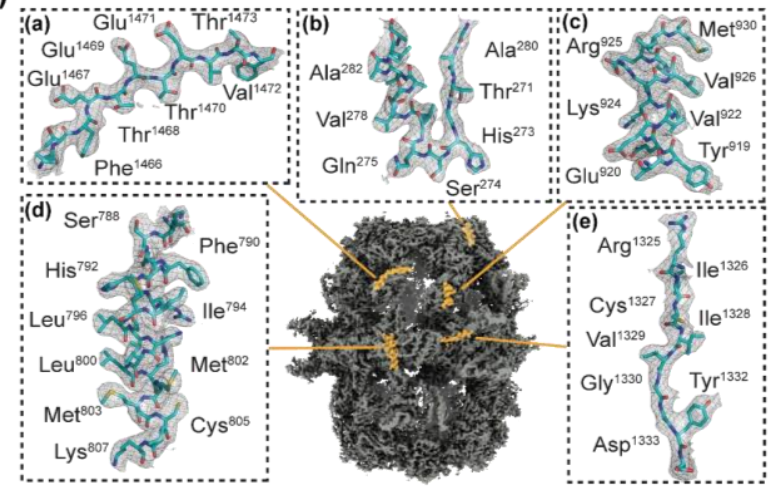

(ii)

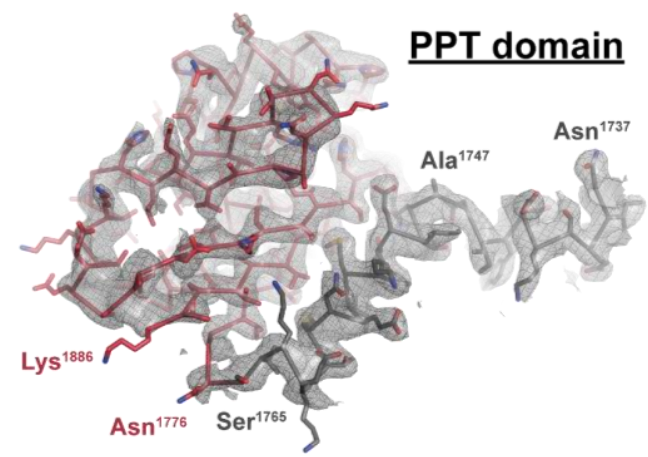

Figure 20. Crystallographic structure of the FAS in the absence of the $\gamma$-subunit. (i) X-ray crystal structure of FAS at $2.9 \AA$ resolution. Several excerpts within the respective enzymatic domains displayed in orange are utilized to show the quality of the final $2 m F o-D F c$ electron density maps contoured at: (a) DH domain $1.5 \sigma$, (b) AT domain $1.5 \sigma,(c)$ ER domain $1.5 \sigma$, (d) KR domain $2.0 \sigma$ and (e) KS domain $2.0 \sigma$. (ii) PPT domain (red) and an $\alpha$-helical sequence (grey) along with their corresponding density contoured at $1.0 \sigma$. These regions were not visualized together with the FAS in previous high resolution structures of this complex (Leibundgut et al., 2007). 
The crystallographic FAS structure was similar in conformation to the non-rotated cryo-EM model. The C $\alpha$ atoms of the crystallographic model and the non-rotated cryo-EM model were compared and had an overall RMSD of $0.92 \AA$ (Figure 21). The first 150 residues of the AT domain along with two segments in the MPT domain (1737-1750, 1850-1970) differed the most between the two models. Upon excluding these residues from the calculations, the RMSD decreased to $0.67 \AA$. To put these variations into context, published structures of identical proteins are known to have a C $\alpha$ atom RMSD in the range of 0 - $1.2 \AA$ (Kufareva and Abagyan, 2012). These deviations are attributed to inherent protein flexibility and experimental resolution limits. Therefore, RMSD values between 0 - $1.2 \AA$ are considered here to infer that the two structures being compared are nearly identical. Furthermore, the overall RMSD between the published crystallographic FAS structure (Leibundgut et al., 2007) and the one from this study is $\sim 1 \AA$, whereas, between the published crystallographic FAS structure and the cryo-EM structure from this study is $\sim 0.7$ (Supplementary Figure S3). The most variations observed in these two comparisons are are also found at the AT and MPT domains. The MPT domain is involved in forming crystal contacts and thus can vary between the EM and crystal structures as well as among the crystals belonging to different space groups. In general, the RMSD values of below $1 \AA$ between the published FAS structure and the ones from this study indicate that the structure of the FAS does not vary significantly between the two structural techniques.

After validating that the structure of the FAS determined by X-ray crystallography and cryo-EM remains almost identical, I then compared the two conformations of the FAS obtained from the endogenous FAS holoenzyme sample and the FAS sample in the absence of the $\gamma$-subunit that were described in the previous sections. 

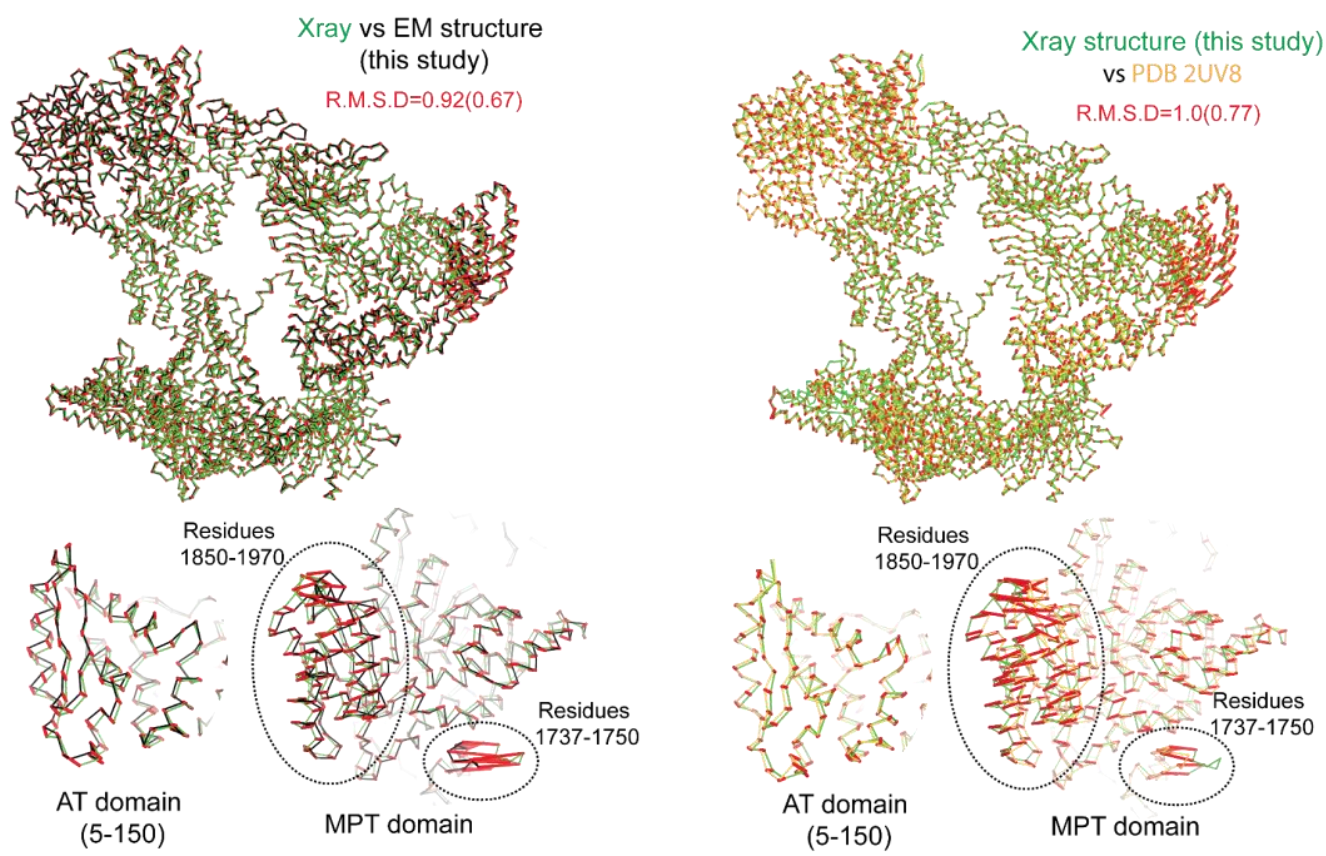

Figure 21. Comparison among FAS models derived from $X$-ray crystallography and cryo$E M$. The RMSD between the cryo-EM and X-ray crystal structure from this study as well as between $X$-ray crystal structure from this study and the published FAS structure (PDB 2UV8) was less than or equal to $1 \AA$. The AT domains residues 5-150 along with MPT domain residues 1737-1750 and 1850-1970 contributed towards 20-30\% of the total deviations among the structures. RMSD values calculated excluding the aforementioned residues are indicated within brackets.

\section{Stabilization of the ACP in the non-rotated conformation using "cycled" FAS}

Unlike in the endogenous FAS holoenzyme complex, the non-rotated conformation of FAS without the $\gamma$-subunit had the ACP domain stalled at the KS domain (Figure 18). This is attributed to the step where FAS was "cycled" using malonyl-CoA and NADPH to drive all the FAS molecules to the same functional state. The stabilization of the ACP domain in the non-rotated state also improved the resolution of this FAS conformation by $0.3 \AA$ for the cryo-EM map. The $2.9 \AA$ crystal structure obtained from these FAS preparations was also $0.2 \AA$ better than the published FAS structures (Jenni et al., 2007; Leibundgut et al., 2007). 


\section{Comparison between rotated FAS conformation with and without the $\gamma$-subunit}

In the absence of the $\gamma$-subunit, FAS was found to adopt both the rotated $(\sim 8 \%)$ and the non-rotated ( $92 \%)$ conformation (Figure 18$)$. The ratio of the two states were different from the endogenous FAS holoenzyme complex, which had $60 \%$ particles in the rotated and $40 \%$ in the non-rotated state (Figure17). Furthermore, the additional densities next to the ER and KR domain were absent in the FAS when the $\gamma$-subunit was absent (Figure 22). The correlation between the presence of the coiled coil density in the structure and the presence of the $\gamma$-subunit in the FAS preparation indicates that these densities corresponds to the $\gamma$ subunit and not to any segment of the FAS itself. The non-rotated conformations of the FAS, both, with and without the $\gamma$-subunit were devoid of any additional densities that might correspond to the $\gamma$-subunit. Therefore, it appears as though the $\gamma$-subunit is bound to the FAS in a stable manner only in the rotated conformation.

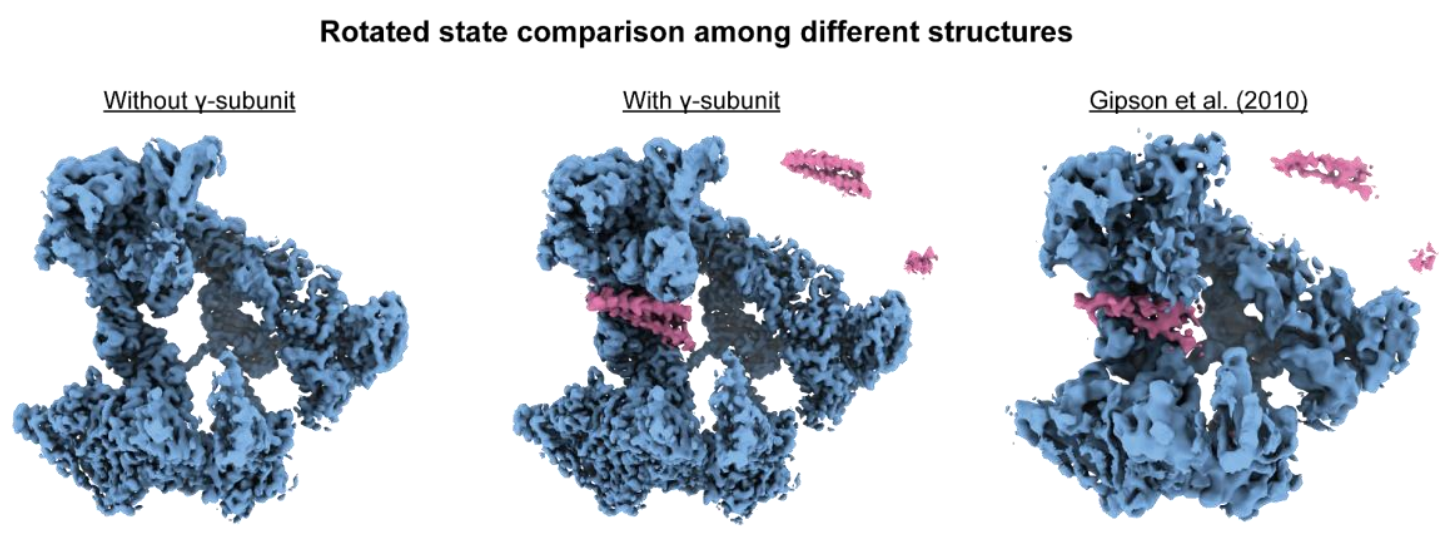

Figure 22. Comparison of rotated FAS conformation of the FAS, FAS-holoenzyme and Gipson et al. (2010) cryo-EM structures. The density maps corresponding to the asymmetric unit of the FAS from the cryo-EM structure of FAS in the absence of the $\gamma$-subunit (left, this study), FASholoenzyme (middle, this study) and the $5.9 \AA$ A FS structure from Gipson et al. (2010) (right). In the presence of the $\gamma$-subunit, the rotated conformation of FAS contains extra densities next to the ER and KR domains (pink). Similar densities were also observed by Gipson et al. (2010).

\subsubsection{Cryo-EM structure of the reconstituted FAS holoenzyme complex}

After having determined the structure of the endogenous FAS holoenzyme complex, I then proceeded to determine the structure of the FAS saturated with the recombinant $\gamma$-subunit. Cryo-EM sample preparation, image acquisition and processing were performed in similar 
manner as described for the endogenous complex (Supplementary Figure S4). 2D classification resulted in 642,703 particles which were then used for 3D classification. All the obtained 3D classes had a rotated conformation with their ACP at the AT site. The absence of the non-rotated conformation of the FAS further supported the previous observation where the presence of the $\gamma$-subunit results in a higher proportion of the rotated conformation. In the end, I was able to reconstruct a cryo-EM structure from 110,597 particles at a global resolution of $2.8 \AA$ with applied D3 symmetry, estimated using the FSC 0.143 criterion. The quality of the FAS holoenzyme structure was comparable to that of the FAS structure and allowed for equally reliable model building (Figure 23, Supplementary Table 3).

(i)

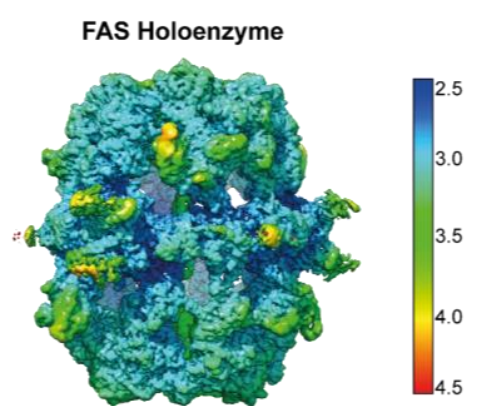

(iii)

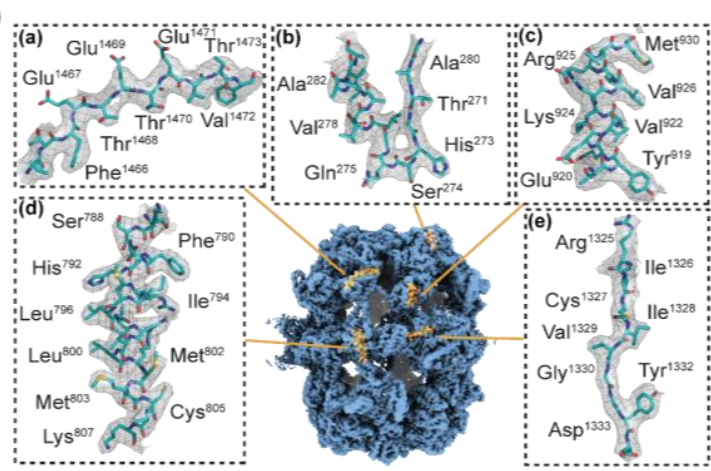

(ii)

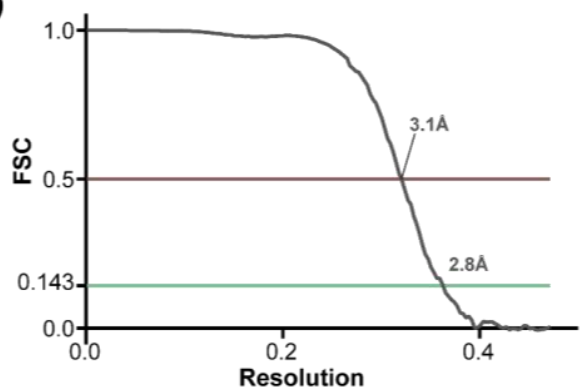

(iv)

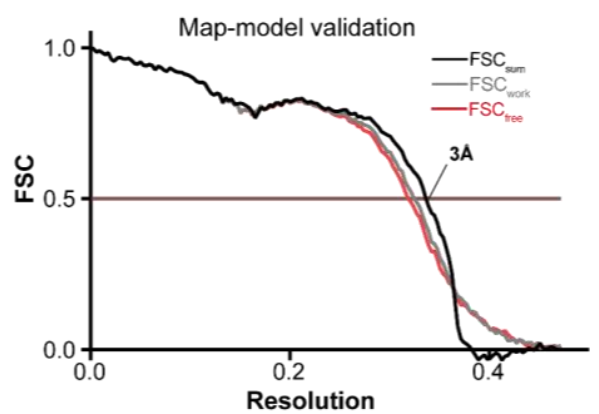

Figure 23. Cryo-EM structure of reconstituted FAS holoenzyme. (i) Local resolution maps of the FAS holoenzyme complex. The resolution range depicted is from $2.5 \AA$ (blue) to $4.5 \AA$ (red). The local resolution varies from $2.5 \AA$ to $3.5 \AA$. (ii) Fourier shell correlation (FSC) of two independently refined subsets of particles of FAS holoenzyme complex. The FSC curve indicates a resolution of 2.8 $\AA$ at a threshold of 0.143. (iii) Several excerpts within (a) DH, (b) AT, (c) ER, (d) KR and (e) KS domains displayed in cyan to show the quality of the final $2.8 \AA$ density map. (iv) Fourier shell correlation (FSC) curves of the final refined map and its model (FSC sum $_{\text {) }}$, first unfiltered half-map and its model (FSC work) as well as the second unfiltered half-map and the model from the first unfiltered half-map $\left(F S C_{\text {free }}\right.$. The FSC $C_{\text {sum }}$ curve indicates a resolution of $3 \AA$ at a threshold of 0.5 . The FSC work and FSC free are nearly identical and indicate that the model is not affected by overfitting. 


\subsubsection{Crystallographic model of the reconstituted FAS holoenzyme complex}

The effect of the $\gamma$-subunit on FAS conformation was also validated using crystals of the $\gamma$ subunit-FAS complex grown by Benjamin Graf (Department of Structural Dynamics, Max Planck Institute for Biophysical Chemistry). They belonged to the primitive monoclinic space group P2 1 with unit cell constants of $a=234.9 \AA, b=430.3 \AA, c=422.6 \AA, \beta=97^{\circ}$, that contained two FAS holoenzyme complexes in the asymmetric unit, and diffracted X-rays to $4.6 \AA$ resolution. I then solved the structure by molecular replacement using the FAS crystal structure to avoid bias. The different FAS domains were then fitted into the density interactively in Coot followed by refinement using Refmac5. The conformation of the FAS in presence of $\gamma$-subunit from the crystallographic data corroborated the findings from cryo-EM data (Figure 24(i)). Along with this, the ACP position and the density corresponding to the $\gamma$-subunit was found to be identical as seen in the EM data and was readily verified by positive difference density- as well as by omit-maps (once the model was built) of the FAS holoenzyme crystal structure (Figure 24(ii)). 
(i)

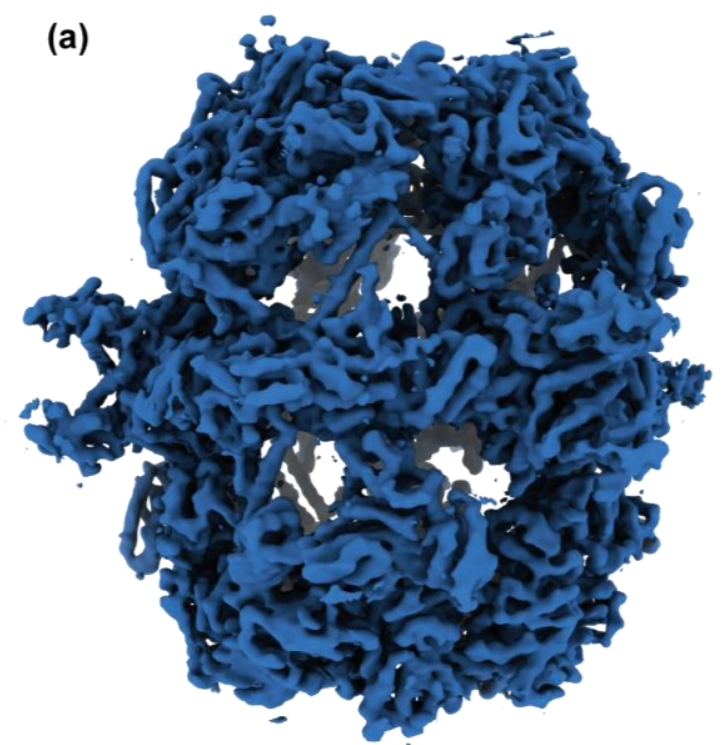

(ii)

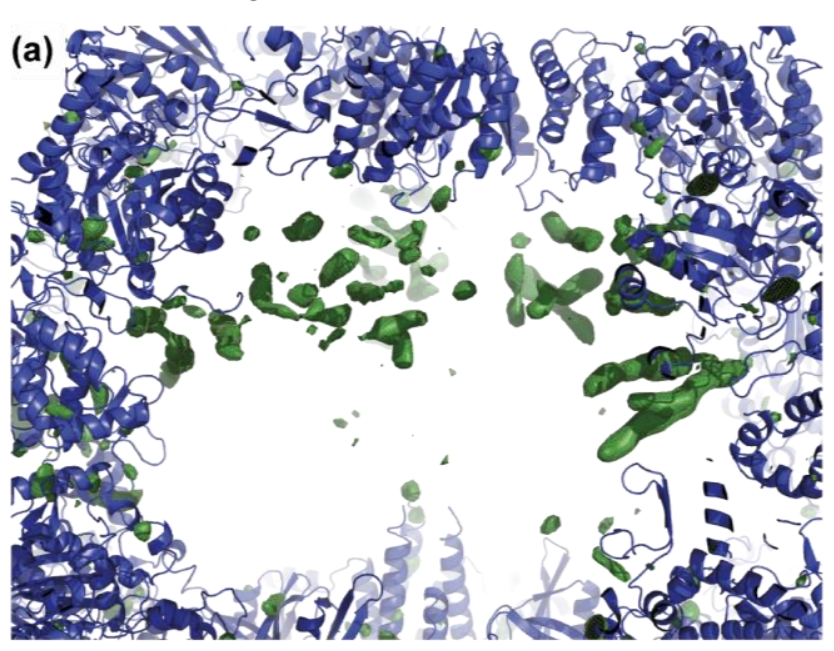

(b)

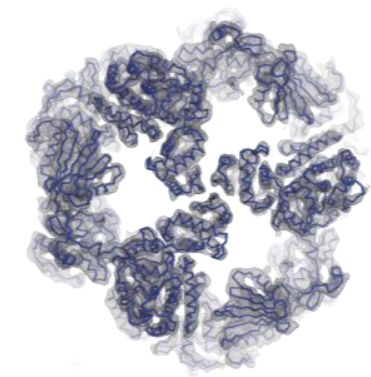

(c)

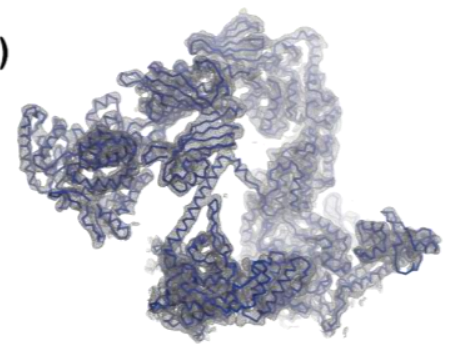

(b)
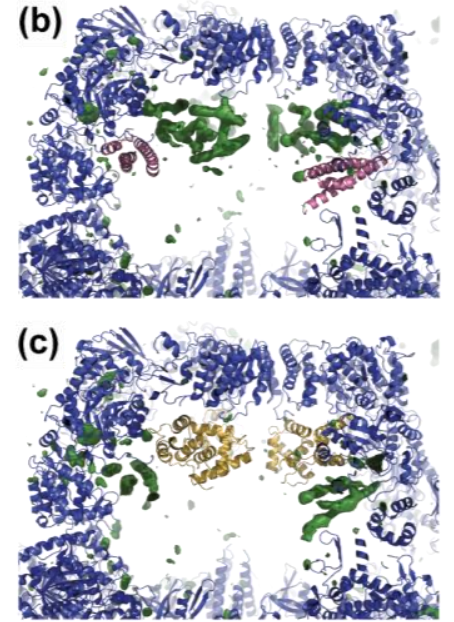

Figure 24. Crystallographic structure of the reconstituted FAS holoenzyme complex. (i) Xray crystal structure of the FAS holoenzyme at 4.6 A resolution. (a) Electron density of the entire FAS holoenzyme molecule as an envelope colored in blue. Also depicted are top (b) and side view (c) of the C $\alpha$-trace models with their corresponding $2 m F o$-DFc electron densities contoured at $1.5 \sigma$. (ii) (a) Shown is the initial positive mFo-DFc difference density (green) contoured at $3 \sigma$ obtained when only $\alpha$-and $\beta$-subunit models are used for refinement of the $4.6 \AA$ FAS holoenzyme crystallographic data. Densities corresponding to $\alpha$-helical segments of ACP domain and $\gamma$-subunit were observed next to the AT and ER domains, respectively. (b) An ACP domain-omit Fo-DFc map is shown contoured at $3 \sigma$ to verify the correct modelling of this domain in the FAS holoenzyme. (c) $A \gamma$ subunit omit Fo-DFc map is shown contoured at $3 \sigma$ to verify the correct modelling of this domain in the FAS holoenzyme. 


\section{Cross-validation between crystallographic and cryo-EM structure of} reconstituted FAS holoenzyme complex

Model of the FAS holoenzyme refined against the cryo-EM map resulted in a model with an overall RMSD of $0.8 \AA$ with respect to the crystal structure. The similarity between the models derived from cryo-EM and X-ray crystallography of both FAS and the FAS holoenzyme, enable a reliable and conclusive interpretation of the structural impact of $\gamma$ subunit binding. These findings are independent of crystal contacts and imposed symmetry in determination of the cryo-EM structures and provide for a robust cross-validation of the structural data.

\section{Correlation of FAS dome structure and ACP location}

In addition to understanding the FAS dynamics in the presence and absence of the $\gamma$ subunit, other general conclusions can also be made from all the crystallographic and cryoEM structures presented in this thesis. FAS adopts a rotated conformation, where the ACP domain is located next to the AT active site (Figure 25(i)). This state corresponds to the first step of the catalytic cycle when the acetyl group is transferred to the ACP domain from the active site Ser274. FAS also adopts a non-rotated conformation where the ACP domain is located at the KS domain or is not visualized for reasons that are poorly understood. The non-rotated conformation of the FAS appears to be sampled during the condensation reaction at the KS active site. Whether the structure of the FAS dome has a rotated or nonrotated conformation when $\mathrm{ACP}$ domain interacts with other active sites $(\mathrm{KR}, \mathrm{ER}, \mathrm{DH}$, MPT) is still unclear. 
(i)

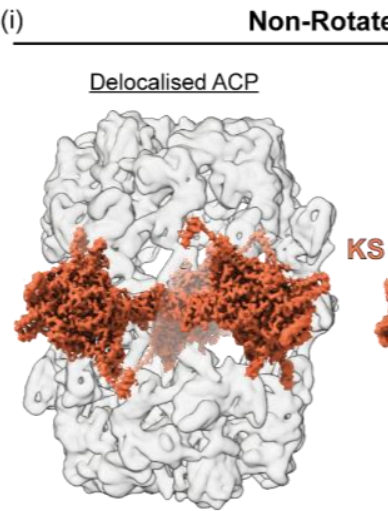

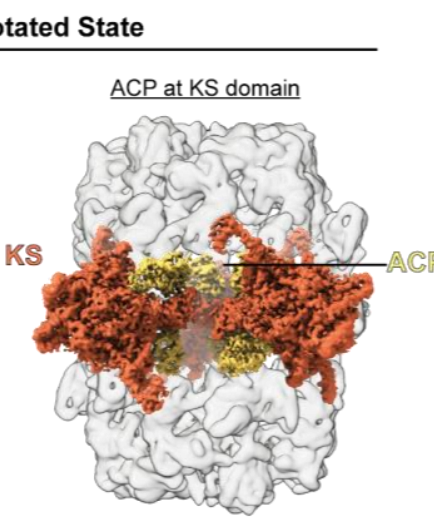

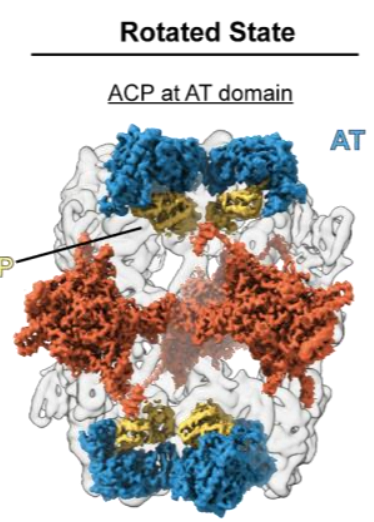

(ii)

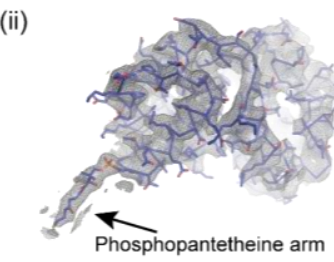

(iii)

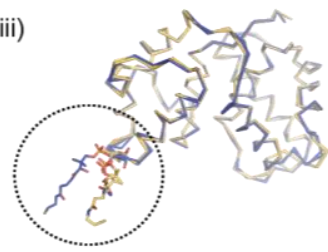

Figure 25. Correlation of FAS dome conformation and ACP domain location. (i) ACP domain (yellow) location within the FAS barrel in the non-rotated and rotated FAS conformation is depicted. In the non-rotated state, the ACP domain can either be delocalized within the FAS dome or be located at the KS domain (orange). In the rotated conformation, the ACP domain is located at the AT domain (blue). (ii) The model and density map of the ACP domain in the $2.8 \AA$ cryo-EM structure of the FAS holoenzyme complex are shown. (iii) Overlay of ACP C $\alpha$-models from the crystal structure of FAS (yellow) and cryo-EM model of the FAS holoenzyme complex (blue). The C $\alpha$-models are nearly identical with a C $\alpha$ RMSD of $0.7 \AA$. The only difference between the two ACP structures appears to be the orientation of the phosphopantetheine prosthetic group (black dotted circle) attached at Ser180.

\section{Why is the ACP domain located at the AT domain only in the rotated FAS conformation?}

The changes in the dome conformation correlate with the movement of the ACP domain from the KS to the AT domain. However, neither the structure of the ACP domain nor the structure of the AT domain differs notably ( $\mathrm{C} \alpha$ atom RMSD of $\sim 0.7 \AA$ ) between the two states. For the ACP domain, only the orientation of the phosphopantetheine prosthetic group protruding into the AT active site was found to be different than when it is in the KS active site (Figure 25(ii)). For the AT domain, upon dome rotation, the AT domains slides downwards through a rigid body shift of $7 \AA$, but this does not result in structural changes within the domain. The structural changes that make ACP binding to the AT domain more favorable appear to lie in the inter domain distance between the AT and ER domains (Figure 26). In the rotated state, the ACP bound at the AT site is not hindered or excluded by the proximal segments of the ER domain. However, in the non-rotated state, two helixes of the ER domain - helix 687-698 and helix 709-723 are situated in a manner that they could hinder the ACP to form productive interactions with the AT domain. Therefore, it can be 
hypothesized that the purpose of the rotation of the AT domain is to shift away from the

ER domain, allowing the ACP to bind unhindered.

(i)

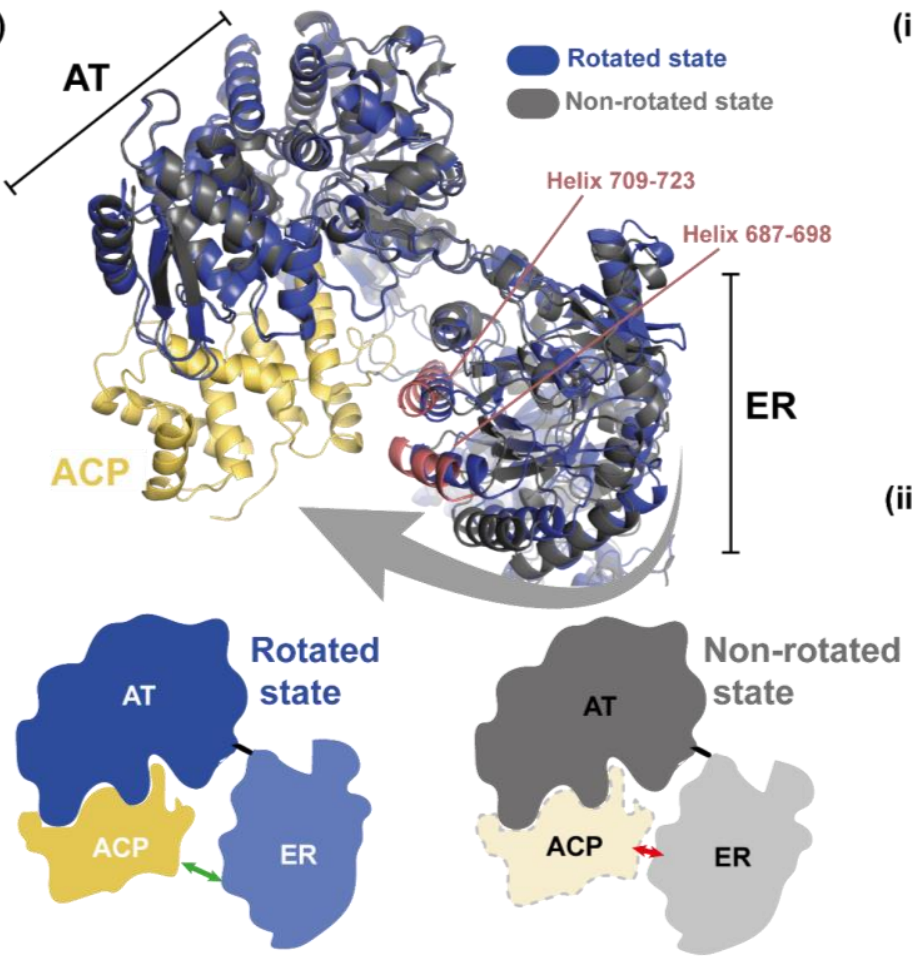

(ii)

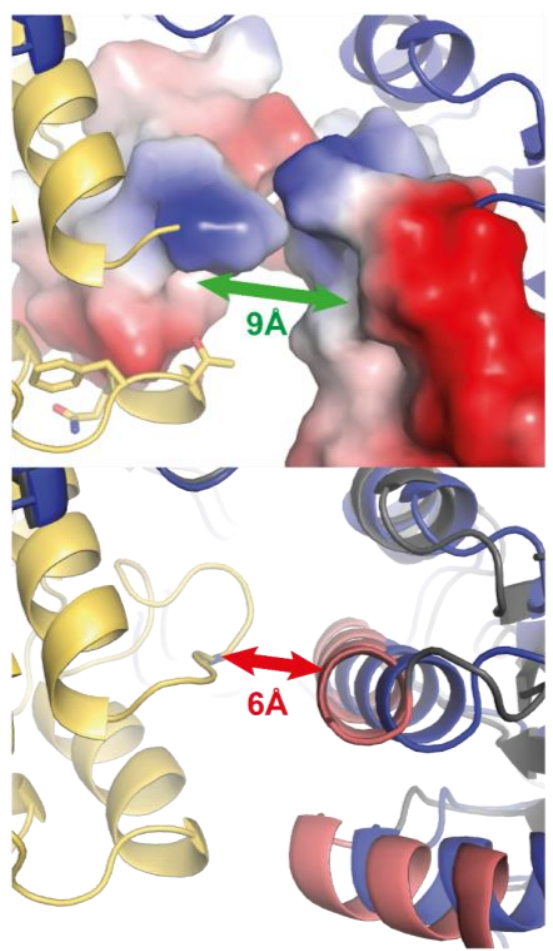

Figure 26. Structural basis for ACP binding to the AT domain in the rotated FAS conformation. (i) Overlay of AT and ER domains of the rotated (blue) and the non-rotated (grey) conformation of FAS where the two structures are aligned with respect to the AT domain. In the rotated state, the ACP (yellow) is bound at the AT domain. In the non-rotated state, the ER domain is located closer to the AT domain in comparison to the rotated state. Assuming that the ACP-AT interactions remain the same in the non-rotated FAS conformation, the two helical segments of the ER domain (salmon) would sterically hinder the binding the of the ACP to the AT domain. Therefore, it is likely that the rotated conformation of the FAS enables the spatial accommodation of the ACP at the AT domain. (ii) Electrostatic interactions between the ACP and ER domain in the rotated FAS conformation are depicted. The positively charge regions of the ACP and ER domain lie in manner that they avoid clashing with each other (iii) Interactions between the ACP and ER domain in the non-rotated FAS conformation assuming the ACP-AT interaction is the same as in the rotated FAS conformation. The helical segment of the (salmon) of the non-rotated ER domain is $\sim 3 \AA$ closer as compared to the rotated ER conformation which might sterically hinder the ACP binding.

\subsubsection{Molecular basis of $\gamma$-subunit - FAS interaction}

After modelling all the domains of the FAS into the $2.8 \AA$ FAS holoenzyme structure, two un-modelled densities corresponding to the $\gamma$-subunit still remained. A large coiled coil like 
density between the ACP and ER domain along with a smaller one in the NADPH binding cleft of the KR domain. For building the model for the $\gamma$-subunit, five homology models were first generated using Robetta server (Kim et al., 2004). The model that fit best to the coiled coil density was then selected as a starting point for interactive building. The density corresponding to the $\gamma$-subunit allowed for distinguishing bulky side chains which were used to maintain the right sequence frame. The structure modelled based on the cryo-EM density was later cross-verified with the XL-MS data (Supplementary Figure S5). Residues 3-59 and 77-113 were placed in the density next to the ER domain and the C-terminal residues 133-150 were fit into the density in the NADPH binding site (Figure 27). Two segments of the $\gamma$-subunit (residues 60-76 and 114-132) were found to be dynamic as they could only be visualized at low density thresholds. Even though these two segments could not be confidently modelled, the XL-MS data clearly suggests that the aspartate rich region (60-76) is in close proximity to the MPT active site and the residues 114-132 form a loop connecting the coiled coil to the C-terminus (Figure 14).

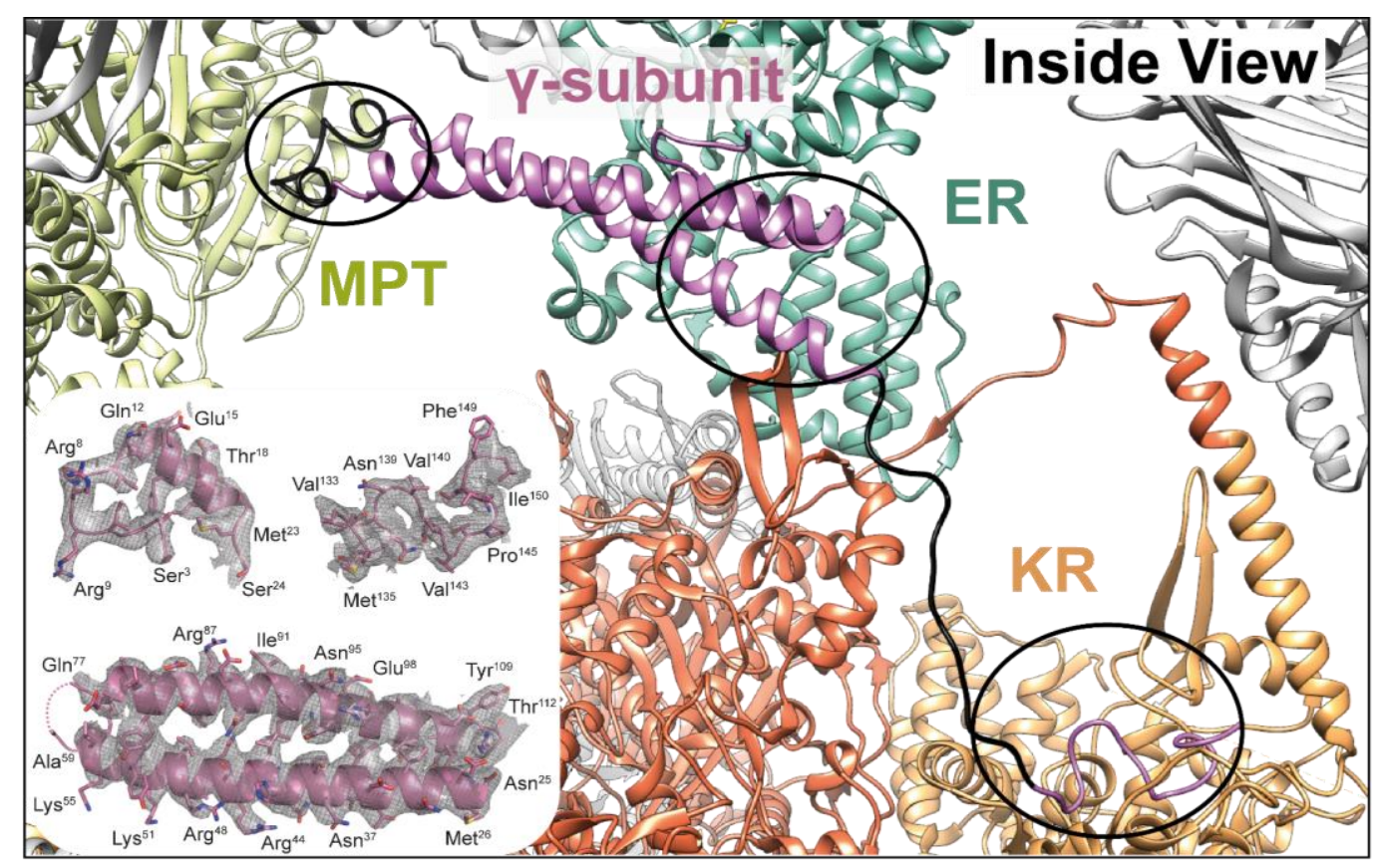

Figure 27. Model of the $\gamma$-subunit. The composite model of the $\gamma$-subunit based on XL-MS data (black) and the observed side chain densities in the FAS holoenzyme cryo-EM structure is depicted. The interactions of the $\gamma$-subunit with the ER (green), MPT (yellow green) and KR (orange) domains are highlighted (circles). In the left inlet, $\gamma$-subunit model placed in its corresponding density can be visualized. No density corresponding to residues 60-76 and 114-132 is visible, most likely owing to their flexible nature. 


\section{Interactions of $\gamma$-subunit with four FAS domains}

Having solved the structure of the FAS holoenzyme complex, an important question I addressed was the molecular basis behind $\gamma$-subunit's effect on FAS activity. Inside the FAS dome, the $\gamma$-subunit spans a distance of $\sim 120 \AA$, where it interacts with four domains (Figure 28) and sterically obstructs substrate binding in three of them -

(1) ER domain: The N-terminal residues (3-24) of the $\gamma$-subunit are situated next to the ER domain occluding the ACP binding site such that Glu14, Glu15 residues protrude into the NADPH binding site. Furthermore, comparing the NADP+ bound ER domain from the Thermomyces lanuginosus FAS structure (Jenni et al., 2007) with that of the FAS holoenzyme complex, side chains of negatively charged $\gamma$-subunit residues Gln13, Glu14 and Glu15 were located within $3 \AA$ of the phosphate group of NADP+. Such an arrangement of the $\gamma$ subunit would sterically hinder the binding of NADPH and would result in a mutually exclusive binding between the $\gamma$-subunit and NADPH.

(2) ACP domain: A positively charged patch on the first helical segment of the $\gamma$-subunit (residues 41-54) is in contact with a complementary negatively charged region on an adjacent helical segment of the ACP domain (residues 207-215). Such charge-based interactions between the $\gamma$-subunit and ACP appear to contribute to the stabilization of the ACP domain at the AT site in the $\gamma$-subunit bound rotated state. When bound at the AT domain, the ACP domain's prosthetic phosphopantetheine arm protrudes into the pocket that is used for binding acetyl-CoA. The substrate binding pocket of the AT site can accommodate either the substrate or the phosphopantetheine arm. Therefore, the stabilization of ACP by the $\gamma$-subunit would hinder the binding of acetyl-CoA and in turn, the transfer of the acetyl moiety from acetyl-CoA to the active site serine. Thus, although the $\gamma$-subunit does not interfere with acetyl-CoA binding site directly, the stabilization of ACP at the AT active site would be a possible hypothesis for the reduction in acetyl-CoA affinity observed during the kinetic analysis.

(3) MPT domain: The aspartate rich loop (residues 59-76) of the $\gamma$-subunit is in close proximity of the substrate binding cleft of MPT domain as evidenced by XL-MS. The MPT substrate binding cleft is lined with positively charged residues such as Arg59, 1861, 1962 
and Lys62, 1918. Specifically, Arg59, 1861, 1962 are important for the malonyl-CoA binding. Structurally, the proximity of the negatively charged loop of the $\gamma$-subunit and the positively charged surface of the MPT domain would result in an electrostatically favorable interaction. Such an interaction would result in (1) the stabilization of the MPT in its rotated state as the non-rotated MPT would sterically exclude the bound $\gamma$-subunit and (2) obstructing malonyl-CoA binding. The presence of negatively charged residues of the $\gamma$ subunit near the malonyl-CoA binding site sterically hinder the incoming malonyl-CoA molecules as well as mask the arginine residues that stabilize the bound malonyl-CoA. These electrostatic interactions therefore are the most likely reason for the 4-fold reduction in malonyl-CoA affinity in the presence of the $\gamma$-subunit.

(4) KR domain: The C-terminus (133-150) of the $\gamma$-subunit is attached to the coiled coil segment via a flexible linker. The C-terminal residues are unstructured but bind within the NADPH binding cleft of the KR domain and would therefore compete with NADPH. This adds another site, where the $\gamma$-subunit interferes with NADPH binding. The disruption of $\mathrm{NADPH}$ binding at the ER and KR domain also correlates with the large decrease in $\mathrm{NADPH}$ affinity during the kinetic analysis seen in the presence of the $\gamma$-subunit.

Thus, the structure of the FAS holoenzyme complex presented here illustrates the molecular basis behind $\gamma$-subunit's ability to stabilize the rotated state of the FAS as well as to affect substrate binding at AT, ER, MPT and KR domain. 


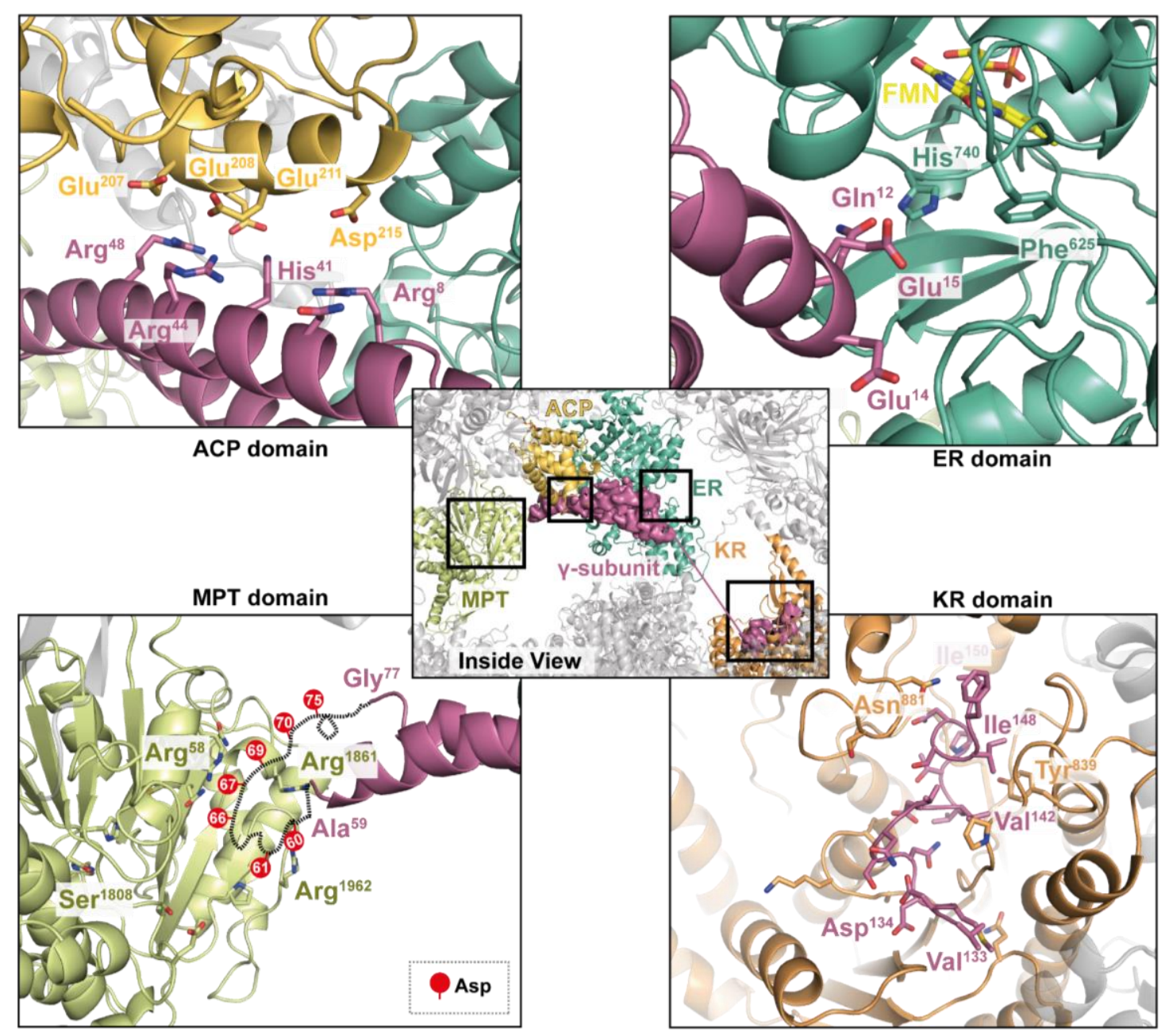

Figure 28. Molecular basis for $\gamma$-subunit-FAS interaction. Shown is the interaction between the $\gamma$-subunit with the ACP domain (top left), ER domain (top right), MPT domain (bottom left) and the KR domain (bottom right). The N-terminal residues of the $\gamma$-subunit interact with the ER domain where negatively charged residues, Glu14, 15, protrude into the NADPH binding site (top right). A patch of basic residues on the first helical segment of the $\gamma$-subunit forms electrostatic interactions with an acidic patch on the proximal helix of the ACP domain (top left). The aspartate rich loop (59-76) of the $\gamma$-subunit is in proximity with arginine residues around the MPT active site cleft (bottom left). The location of this $\gamma$-subunit segment was confirmed using XL-MS. The Cterminal residues are unstructured but bind into the NADPH binding site on the KR domain active site (bottom right).

\subsubsection{Changes in the conformational landscape of FAS due to the $\gamma$ - subunit}

Until now, this thesis has only dealt with the most dominant conformations of the FAS, with and without the $\gamma$-subunit. However, proteins exist in an ensemble of conformations, where only some are stable enough to be resolved at high resolution. To complement the 
observations of the effect of the $\gamma$-subunit on the ratio of rotated and non-rotated state of FAS, I investigated the effect of such protein-protein interactions on the conformational landscape of FAS. Such a comprehensive structural analysis would allow insight into the global implications of the binding of the $\gamma$-subunit to the FAS.

For this analysis, $0.5 \mu \mathrm{M}$ FAS was titrated with increasing concentration of the $\gamma$-subunit before grid preparation for cryo-EM. It is important to note here that even though the structures of FAS and FAS holoenzyme complex were determined using grids with a thin carbon support film, the very high concentrations of $\gamma$-subunit used for energy landscape calculations prevented the binding of FAS to the support film (data not shown). Thus, the samples were frozen in the absence of any kind of support film attached to the grids. Small datasets were then acquired for the different samples (Supplementary Table 5). Using the obtained data, conformational landscapes of FAS in the presence of different $\gamma$-subunit concentrations were determined using 3D principle component analysis (PCA) followed by energy landscape calculations (Haselbach et al., 2017, 2018).

First, I refined 3D structures of the individual datasets with a mask corresponding to the central wheel, which is indistinguishable in conformation in both the non-rotated and rotated. To gain insight into the conformational space occupied by the dome in each individual dataset, I then performed a 3D classification without alignment in batches of 6,500 particles each, relying exclusively on the alignment parameters of the central wheel obtained from the focused 3D refinement. The individual 3D classes thus obtained were individually refined to ensure their validity. At this step, quantification of particles belonging to $3 \mathrm{D}$ classes corresponding to either the non-rotated or rotated conformation showed a clear correlation between the dome conformation and abundance of $\gamma$-subunit (Figure 29(i)). In conditions without the $\gamma$-subunit, 2\% of the particles adopt a rotated conformation. This increased to $58 \%$ at $1 \mu \mathrm{M}$ then to $88 \%$ at $3 \mu \mathrm{M}$ and $>98 \%$ at saturating concentrations of the $\gamma$-subunit. Next, a total of 165 3D volumes were used for 3D principal component analysis (PCA). The first eigenvector of this 3D PCA described the compaction of the dome, whereas the second eigenvector signified the notable rotation of the dome. By using the number of particles contributing to a particular 3D class, an energy landscape was 
mapped by performing a Boltzmann interpolation within a coordinate system that is defined by the first and second eigenvectors of the PCA.
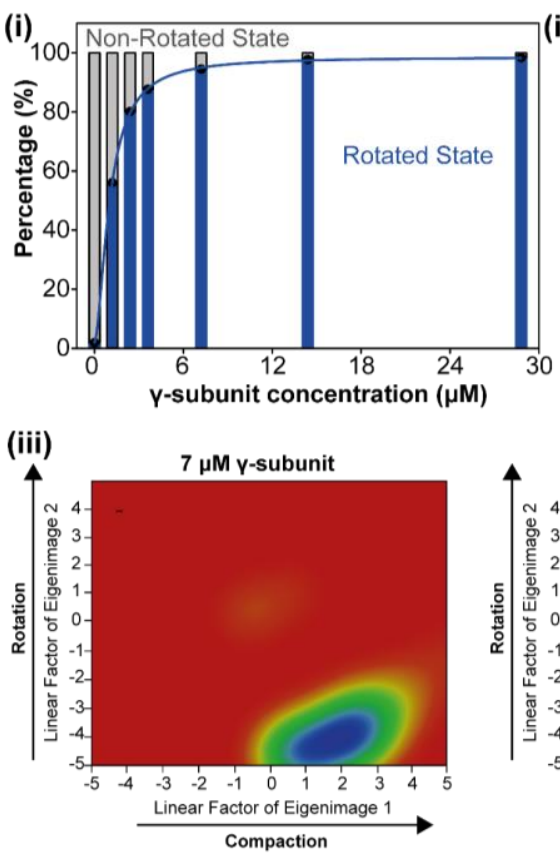

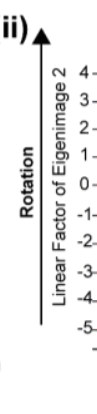

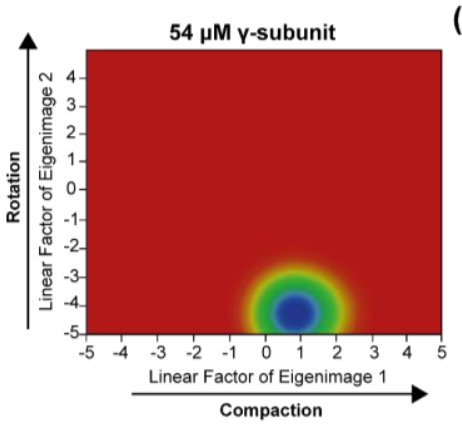

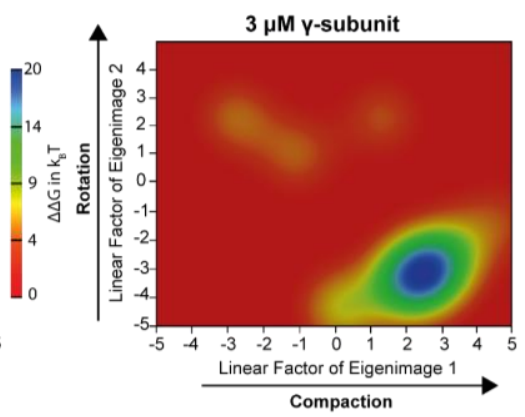

(iv)

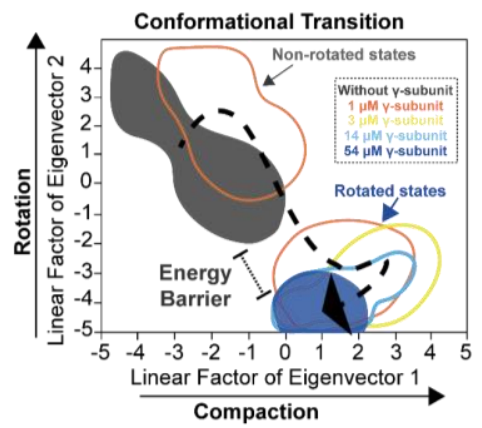

Figure 29. Effect of the $\gamma$-subunit on the conformational landscape of the FAS. (i) Analysis of the proportion of particles in the non-rotated or rotated conformation in response to increasing amounts of the $\gamma$-subunit. While less than $2 \%$ of particles adopt the rotated state in the absence of the $\gamma$-subunit in a $0.6 \mu M$ FAS solution, their proportion gradually increases to $56 \%$ at a $1 \mu M \gamma$ subunit, to $80 \%$ at a $2 \mu M \gamma$-subunit, to $88 \%$ at a $3 \mu M \gamma$-subunit and finally saturates at about 95\% at $7 \mu M \gamma$-subunit concentration. (ii) Energy landscape analysis of FAS conformations induced by the $\gamma$-subunit. The $x$-axis of each individual plots corresponds to the linear factor of the first Eigenimage, which describes the overall compaction of the FAS structure. The y-axis corresponds to the linear factor of the second Eigenimage, which describes the rotation of the FAS dome. The FAS adopts a non-rotated conformation (left). At $3 \mu M \gamma$-subunit, a minor population of the FAS particles adopt a non-rotated conformation whereas the majority have shifted to a rotated one (right). (iii) At $\gamma$-subunit concentration in excess of $3 \mu M$, FAS particles adopt only the rotated conformation. (iv) Overlay of the energy landscape plots of the FAS in the presence of increasing concentrations of the $\gamma$-subunit. The energy landscapes indicate that binding of the $\gamma$-subunit results in the formation of an energy barrier (dotted line) at $4{ }^{\circ} \mathrm{C}$ to gain access to the non-rotated conformation and suggests a smooth transition with some additional conformational intermediates. The path of transition from non-rotated to rotated FAS conformations is indicated by the dotted black line.

The energy landscape of the FAS indicate that although all particles have a non-rotated state, there exists one major conformational minimum along with small number of particles 
with conformations deviating due to minor rotation and compaction of FAS molecules (Figure 29(ii)). At $1 \mu \mathrm{M} \gamma$-subunit concentration, two minima exist, one representing the non-rotated state and the other being the $\gamma$-subunit bound rotated state. At higher $\gamma$ subunit concentrations, there is again one major conformational minimum, albeit for the rotated state of the FAS. Moreover, the energy landscapes indicate that binding of the $\gamma$ subunit results in the formation of an energy barrier restricting access to the non-rotated conformation (Figure 29(iv)). These energy landscapes recapitulate the binding of the $\gamma$ subunit and the ensuing formation of the FAS holoenzyme complex results in the stabilization of the rotated conformation.

\subsection{Other tools tested for stabilizing the FAS structure}

Along with chromatography-free purification, cycling the FAS complex during purification and GraFix, I also investigated whether FAS substrates or anti-FAS nanobodies could be used for stabilizing the FAS structure.

\subsubsection{Substrate induced structural changes of the FAS}

A cryo-EM dataset of 11,416 micrographs was acquired on a Titan Krios with Falcon III detector (integrating mode) for the FAS in presence of malonyl-CoA and NADPH (Supplementary Table 3). Upon 3D classification, only $2.6 \%$ of the particles had the dome in a rotated state whereas the rest were in the non-rotated form. In comparison, FAS alone has only $\sim 8 \%$ of the particles with a rotated dome conformation (section 3.2.5.3.). In the end, even though only $2.6 \%$ of the particles had a rotated conformation, a $3.3 \AA$ structure of the rotated form using 43,467 particles owing to the large initial dataset of 1.7 million particles. Furthermore, a $3.0 \AA$ structure for the non-rotated form using 191,899 particles was obtained. At these resolutions, it was possible to unambiguously distinguish the bound substrates as well as model them into their corresponding densities (Figure 30). 


\subsubsection{Substrate binding is conformation independent}

In the presence of malonyl-CoA and $\mathrm{NADPH}$, the ratio of rotated/non-rotated state decreased by $5 \%$ as compared to when they are absent. One might expect that this would indicate preferential binding of the substrates to the non-rotated state of FAS; however, this was not the case. The substrate binding was found to be independent of the dome conformation as unambiguous densities corresponding to malonyl-CoA and NADPH were observed in both FAS conformations. However, substrate binding to the FAS did result in local conformational changes in the FAS domains (Figure 30).

\subsubsection{Local changes in FAS domains upon substrate binding}

KR domain: The loop segment 874-881 of the $\alpha$-subunit located next to the KR domain active site is not well defined in the cryo-EM maps (Figure 30(ii)). However, when NADPH is bound to the KR active site pocket, the loop segment $874-881$ clamps onto the substrate. This structural rearrangement probably helps in stabilizing the bound substrate. This has also been shown in the NADP+ bound FAS structure from Thermomyces languinosus (Jenni et al., 2007). This similarity in NADPH/NADP+ induced changes in the KR domain among the two species further emphasizes how structural mechanisms of this complex have remained conserved despite the divergence of the sequences over the course of evolution.

MPT domain: The binding of malonyl-CoA also results in a conformational change in the MPT domain. The helical segments on the right side of the MPT catalytic cleft (residues 1850-1970) encloses upon the substrate like the claw of a crab (Figure 30(iiia)). This conformational change of the MPT results in the formation of polar contacts between the arginine residues 59 and 1961 and the phosphate groups of malonyl-CoA which would have a stabilizing effect on the bound substrate. 
(i)

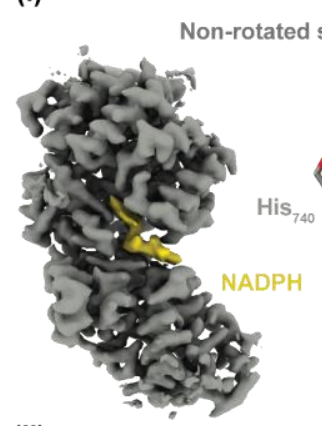

(ii)

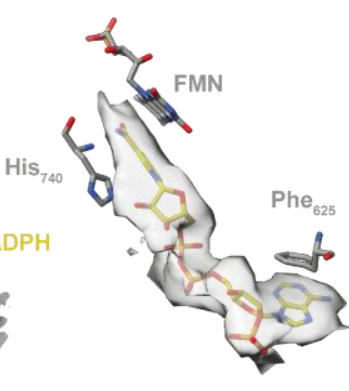

$\underline{\text { KR domain }}$

With NADPH
ER domain

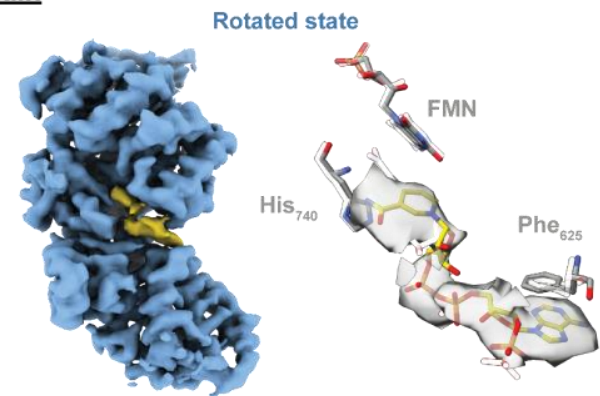

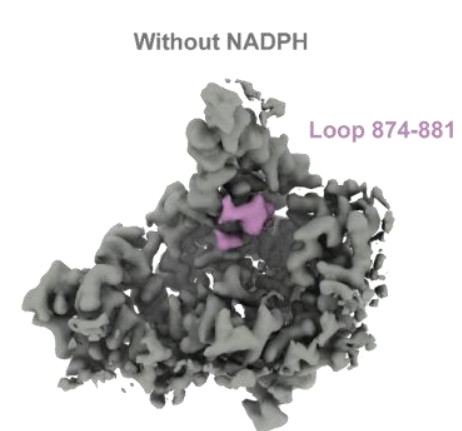

(iii)

(a)

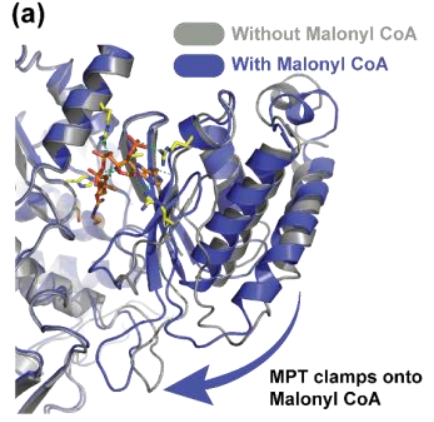

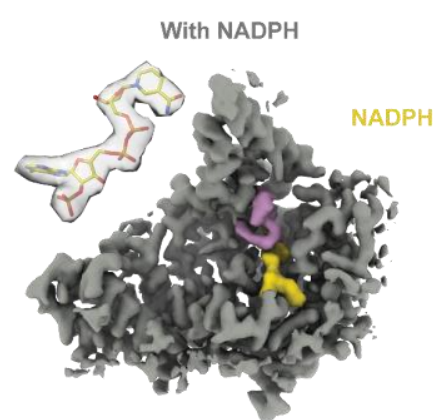

MPT domain

(b)

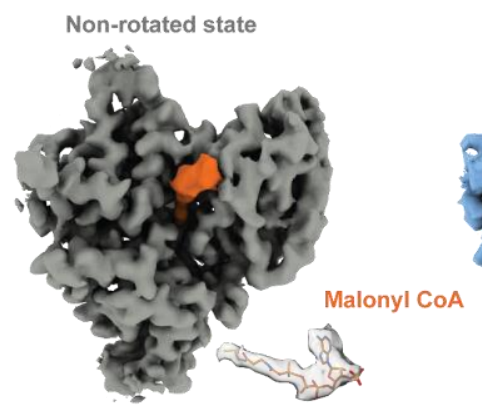

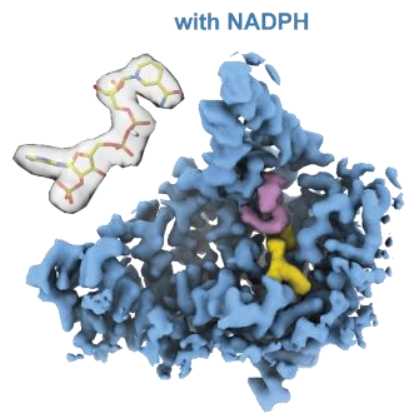

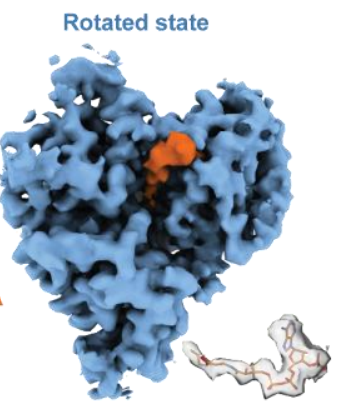

Figure 30. Structural changes in the FAS in the presence of malonyl-CoA and NADPH. (i) Density for the ER domain with bound NADPH (yellow) in the non-rotated (grey) and rotated (blue) FAS conformation. The experimental density for modelling NADPH molecules along with surrounding active site resides of the ER domain is shown beside the respective domains. On the right, an overlay of the ER active site residues of the ER domain in the non-rotated state (white) with those from the rotated FAS conformation are shown.(ii) Experimental density for the KR domain in the absence of NADPH (left, grey) as well as in the presence of NADPH in the KR active site in the non-rotated (middle, grey) and rotated (right, blue) FAS conformation are shown. The flexible segment (874-881) near the active site (pink) clamps onto the bound NADPH molecule (yellow). (iii) (a) Overlay of the MPT domain in the absence (grey) and presence (blue) of a bound malonylCoA molecule (orange). Binding of the malonyl-CoA elicits a large structural change in the $\alpha$-helical segments on the right of the active site where they clamp down on the bound substrate. (b) Experimental density for malonyl-CoA bound to the MPT domain in the non-rotated (grey) and rotated (blue) FAS conformation is depicted. 


\subsubsection{Effect of dome rotation on NADPH binding to the ER domain}

Unlike for the KR and MPT domain, substrate binding to the ER domain was not similar between the two FAS conformations (Figure 30(i)). The NADPH binding pocket of the ER domain differs between the rotated and non-rotated FAS state. In the rotated state, ER active site pocket is narrower by $\sim 1 \AA$ than in the non-rotated state. These minor changes in the ER domain appear to change the conformation of the bound NADPH. In the non-rotated state, NADPH is situated such that its nicotinamide ring is located between the catalytic His740 and the FMN ring. The bound NADPH is in close proximity of both the catalytic residues of the ER domain. This has also been shown in the NADP+ bound FAS structure from Thermomyces languinosus (Jenni et al., 2007). In contrast, in the rotated state, the nicotinamide ring flips out by $\sim 45^{\circ}$ towards the inside of the FAS dome. The distance between the flipped-out nicotinamide ring and the FMN is $\sim 6 \AA$. At such a distance it is unlikely that the FMN can catalyze the reaction, where reducing equivalent from NADPH are used to reduce $\beta$-enoyl acyl chains. These results show that although NADPH molecules can bind the ER domain in the rotated and non-rotated conformations of the FAS, the bound $\mathrm{NADPH}$ is in close proximity to the catalytic residues only in the non-rotated state of the FAS. Since it is not possible to distinguish between NADPH and NADP+ in the cryo-EM maps at a resolution of $3 \AA$, additional experiments would need to be performed to determine the reduction state of the NADPH. Furthermore, the biological significance of the "flipped-out" conformation of NADPH would also need to be examined to understand which NADPH conformation is required for catalysis.

\subsubsection{Comparison between the FAS holoenzyme complex and the rotated FAS conformation in the presence of malonyl-CoA and NADPH}

The structure of the rotated FAS conformation with the bound malonyl-CoA and NADPH (NADP+) was also compared to the FAS holoenzyme structure. It was found that all interactions between the $\gamma$-subunit and the ER, MPT and KR domain, as suggested in section 3.2.5.5., would indeed obstruct the binding of the substrates (Figure 31). The Nterminal residues of the $\gamma$-subunit, Gln 12, Glu 14 and 15 form steric clashes with the ER bound NADPH. The residues at the MPT which stabilize the bound malonyl-CoA, Arg 59, 
1961 are present in the region which forms short range contacts with the negatively charged loop of the $\gamma$-subunit as shown by the XL-MS data. These residues would be masked by the $\gamma$-subunit rendering them unavailable for malonyl-CoA. Finally, the NADPH bound at the KR site overlaps with the C-terminus of the $\gamma$-subunit which again points toward steric exclusion of this substrate upon $\gamma$-subunit binding to the FAS.

(i)

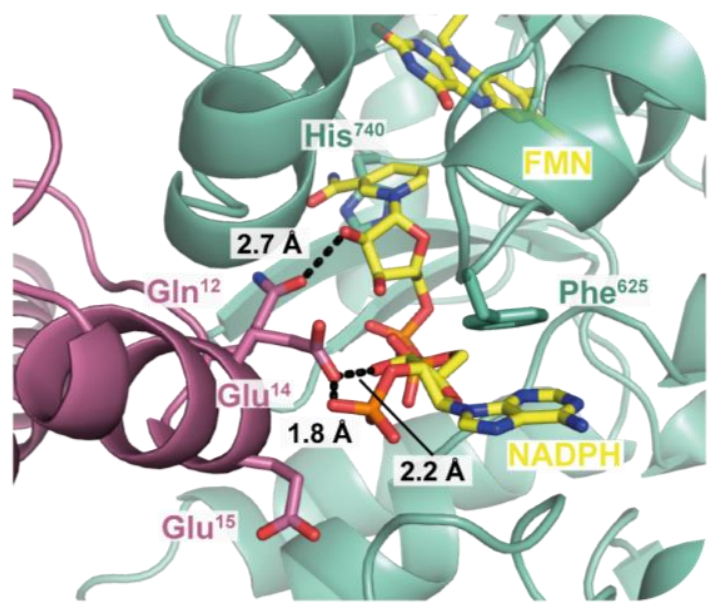

(ii)

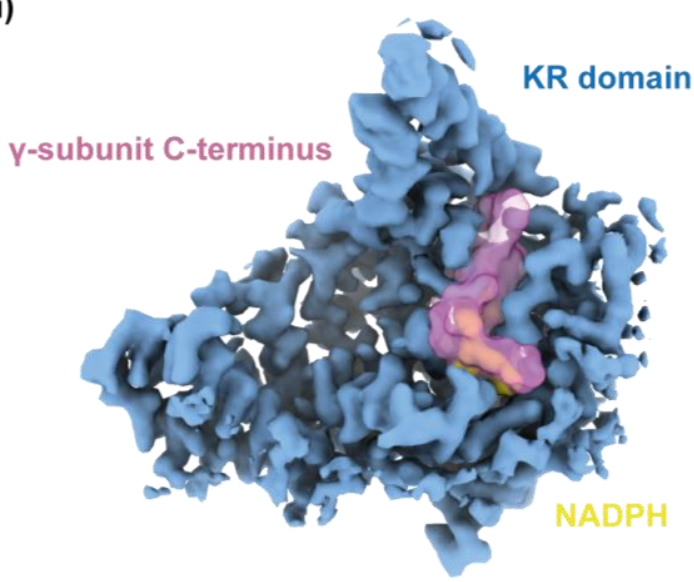

Figure 31. Steric exclusion of substrates by the $\gamma$-subunit. (i) Overlay of the $\gamma$-subunit from the FAS holoenzyme structure and the NADPH bound ER domain from the rotated FAS conformation. The N-terminal residues of the $\gamma$-subunit, namely Gln12, Glu14 and 15, would sterically hinder the binding of NADPH. (ii) Overlay of the C-terminus of the $\gamma$-subunit from the FAS holoenzyme structure and the NADPH bound KR domain from the rotated FAS conformation. The C-terminal residues of the $\gamma$-subunit occlude the NADPH binding site of the KR domain.

\subsubsection{Conformational stabilization using anti-FAS nanobodies}

The discovery of the $\gamma$-subunit and its ability to stabilize the rotated conformation of FAS was serendipitous. To stabilize other conformations of the FAS where the ACP domain might be stalled at sites except the AT or KS domain, I evaluated the use of anti-FAS nanobodies. This idea was inspired by the use of nanobodies to select for distinct conformations of complexes like GPCRs (Dmitriev et al., 2016; Steyaert and Kobilka, 2011) and I wanted to test if this might also hold true for a large multimeric complex like the FAS. 


\subsubsection{Characterizing the nanobody clones}

Immunization of alpacas with CtFAS and ScFAS as well as selection of yeast FAS specific nanobodies was done in collaboration with Tino Pleiner from the lab of Dirk Görlich (Max Planck Institute of Biophysical Chemistry) as previously described (Pleiner et al., 2015). The nanobody clones selected after three rounds of phage display were grouped into 10 classes based on the sequence similarity of their CDR III region. I then expressed and purified one representative member from the different classes as described by Pleiner et al. (2015) for characterizing their binding to the FAS.
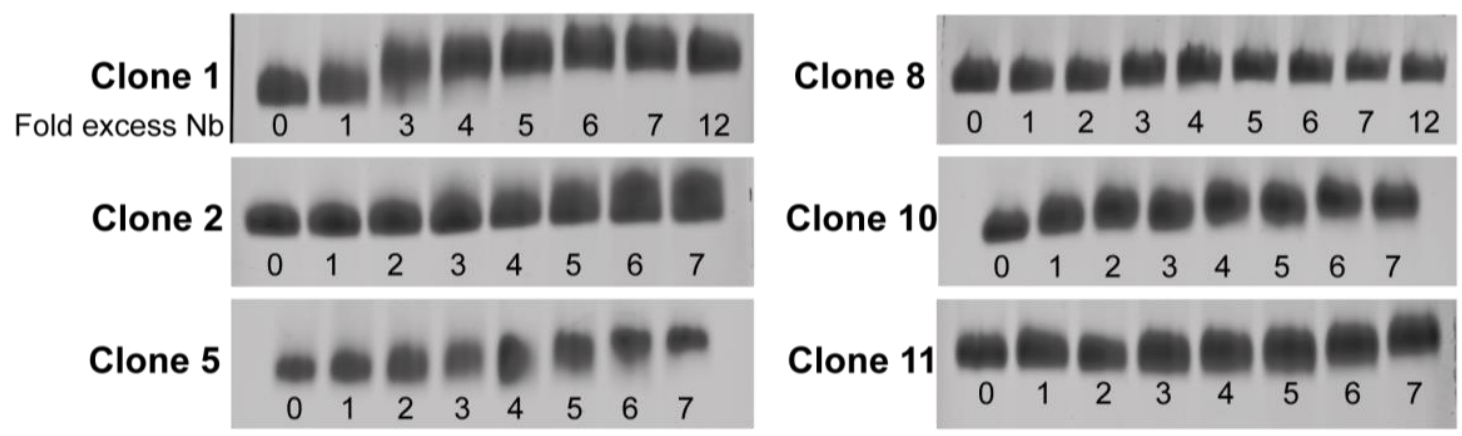

Figure 32. Band shift assay to identify FAS-nanobody complex. Shown is the effect of increasing nanobody concentrations (in molar fold excess) on the migration of FAS in a native agarose gel. The binding of the respective nanobody to the FAS changes the molecular weight and net charge on the FAS molecule which is elicited as a band shift.

Band shift assays were conducted on $1 \%$ native agarose gels for testing whether the isolated nanobody clones bind the FAS. Increasing molar ratios of the different nanobody clones were titrated against a constant FAS concentration. FAS migration was reduced upon increasing the concentrations of the nanobodies eliciting as a band shift (Figure 32). All the nanobody clones tested were found to bind to the FAS. For most of the tested clones, binding saturation was reached in the presence of $\sim 7$-fold molar excess of the respective nanobody indicating strong binding affinities. 


\subsubsection{Mapping the binding sites on FAS}

To map the binding sites on the FAS, small cryo-EM datasets of Nb-FAS complexes from the 8 most distinct classes were acquired. The samples prepared were in the presence of malonyl-CoA and NADPH to reduce the FAS dome dynamics so that better resolutions could be attained with the limited amount of data. The datasets ranged from $10 \mathrm{~K}-200 \mathrm{~K}$ particles. In, the end, a total of five different binding sites were found - three at the MPT domain, one at the $\mathrm{DH}$ domain and one at the AT domain (Figure 33).

(i)

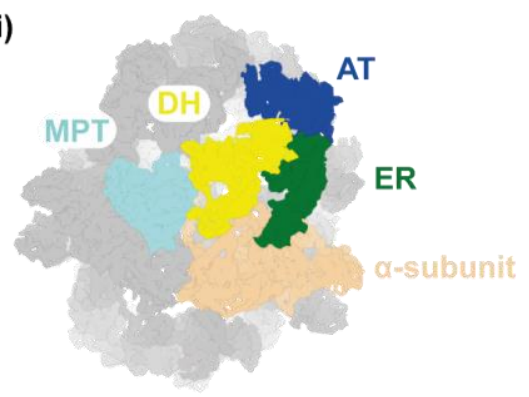

(ii)

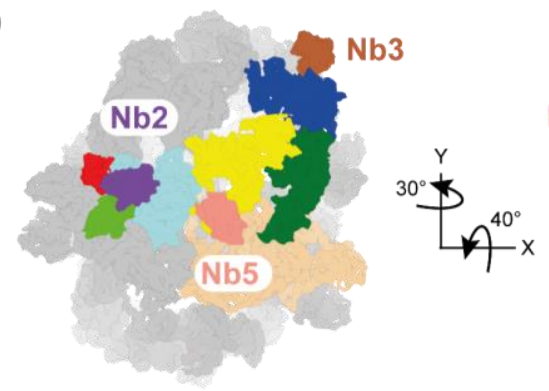

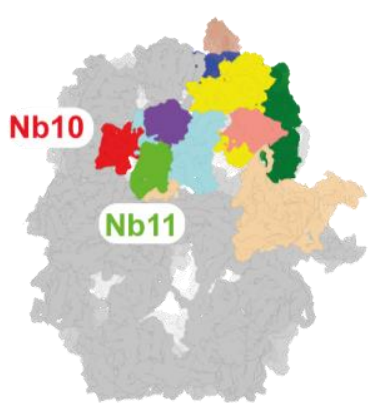

Figure 33. Unique nanobody binding sites mapped on the FAS. (i) Domain organization of the $\beta$-subunit of the FAS is depicted. (ii) Shown are the unique binding sites of anti-FAS nanobodies generated in this study. Nb2 (purple), Nb3 (brown), Nb5 (salmon), Nb10 (red) and Nb11 (green) on the FAS complex. Nb2, 10 and 11 bind to different parts of the MPT domain (cyan). Nb3 binds to the AT domain (blue) whereas the Nb5 binds to the segment of the DH domain (yellow) next to the MPT domain.

\subsubsection{Effect on stabilizing FAS conformations}

In the presence of substrates, all $\mathrm{Nb}$-FAS complexes were in the non-rotated conformation. This was expected as the FAS mainly adopts the non-rotated state on addition of substrates (section 3.3.1.). Along with this, the ACP domain was also situated at the KS domain for all except $\mathrm{Nb5}$. In Nb5-FAS complex, a poorly defined ACP domain density was observed next to the MPT domain (Figure 34(i)). Since, the dehydratase domain bound Nb5 is situated in close proximity of the MPT domain, it is possible that Nb5 modulates the MPT domain in a way that promotes ACP-MPT domain interactions over those between the ACP domain and the KS domain (Figure 34(ii)). It is also important to mention here that the malonylCoA present in the sample would compete with the ACP to bind to the MPT resulting in 
destabilizing the ACP-MPT interactions. This might be a reason why the ACP domain density is fragmented. Therefore, it would be necessary in the future to test whether Nb5 could stabilize the ACP domain at the MPT domain in the absence of substrates.
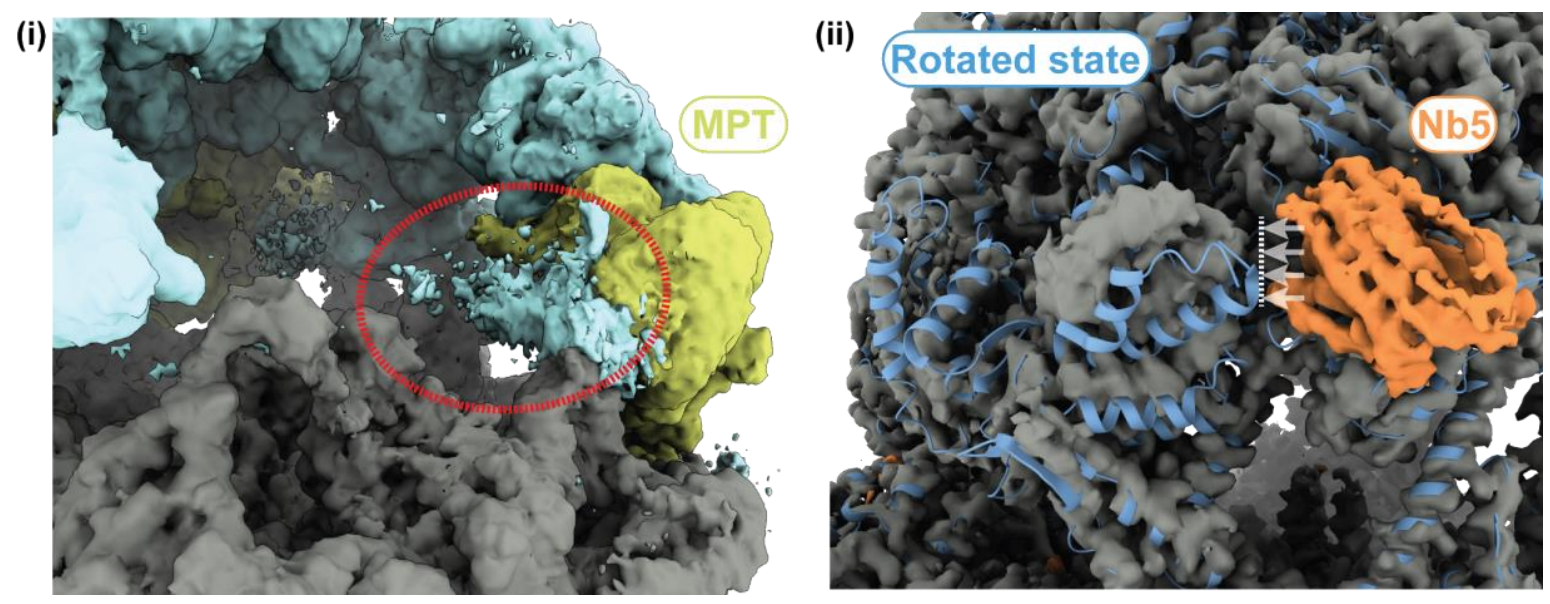

Figure 34. Effect of Nb5 binding to the FAS structure. (i) The view inside the dome of FAS bound $N b 5$ is shown. Density corresponding to the ACP domain is absent from the KS domain. However, low-resolution densities which most likely correspond to the ACP domain (red dotted circle) are present next to the MPT active site (green). (ii) Overlay of the experimental density of the Nb5 bound FAS with the atomic model of the rotated conformation of the FAS. Nb5 is bound adjacent to the MPT domain in a manner that it can restrict the movement of the MPT. Since the DH domain is nearly identical between the non-rotated and rotated FAS conformations, the Nb5 location is also likely to remain constant between the two conformations. In this scenario, the MPT conformation in the rotated state of the FAS would be sterically hindered by Nb5 (white arrows).

Additionally, the effect of $\mathrm{Nb} 2$ and $\mathrm{Nb3}$ on FAS conformations were tested in the absence of substrates. Both of these clones were able to bind the rotated as well as the non-rotated FAS. However, the ratio of these two conformations was different from what we observe for FAS alone. In $\mathrm{Nb} 2$ - and Nb3-FAS complexes, $\sim 48 \%$ and $\sim 11 \%$ of the particles were in the rotated state, respectively (Figure 35). In comparison, FAS alone has only $\sim 8 \%$ of the particles in this conformation. Therefore, this initial test with the two clones suggests that not only can these nanobodies bind to the different conformational states, they somehow can also stabilize the rotated form of FAS.

These preliminary results regarding the use of nanobodies as a tool for structural studies of large macromolecular complexes are promising. Future prospects on the how to utilize the 
different anti-FAS nanobody clones generated as a part of this study will be discussed in more detail in the next section.
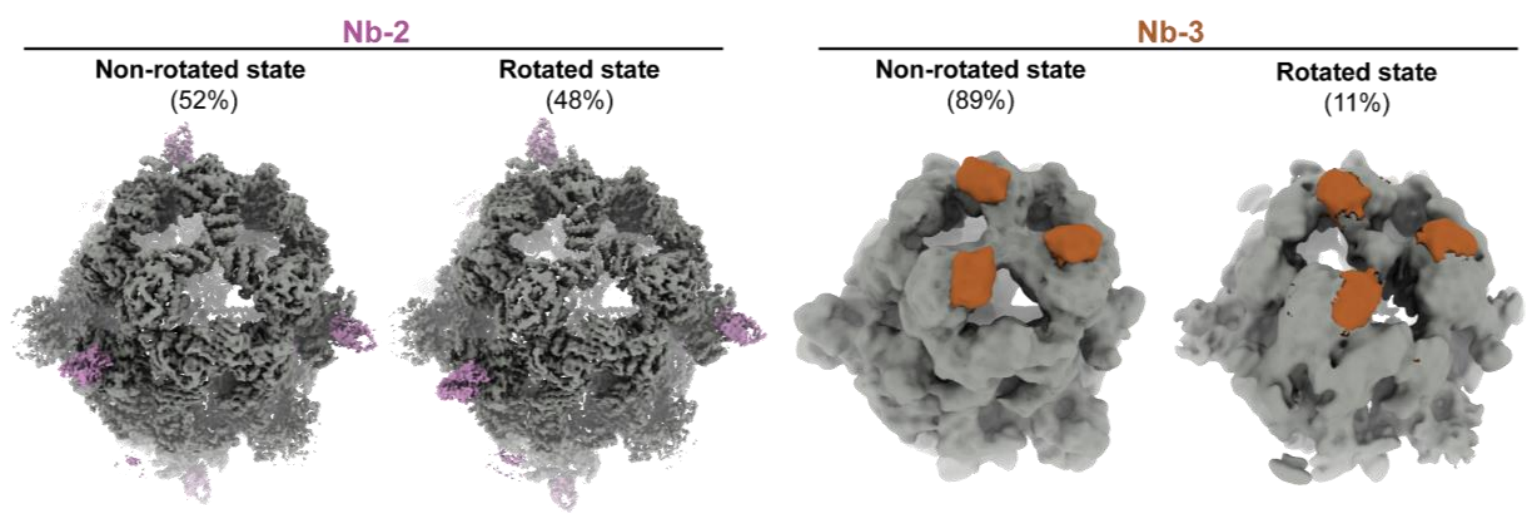

Figure 35. Stabilization of FAS conformations on nanobody binding. Shown are the nonrotated and rotated conformation of the FAS in the presence of Nb2 (left) and Nb3 (right). The relative abundance of the respective conformations are mention within parenthesis. As compared to the FAS in the absence of nanobodies where only $8 \%$ of the particles adopt a rotated conformation, $48 \%$ of the Nb2 bound FAS particles and 11\% of the Nb3 bound FAS particles were in the rotated conformation.

\subsubsection{Future uses for anti-FAS nanobodies}

\section{Nanobodies for FAS purification.}

The specificity and high affinity of anti-FAS nanobodies can be used for purifying fungal type I FAS from different species. Structural conservation along with limited amount of sequence conservation among the different fungal species would allow these nanobodies to also bind FAS from other fungal species. Since cryo-EM methods for high resolution FAS structure determination require only picomole quantities of FAS, small amounts of cell lysate can then be used for purifying FAS from different species. This would be especially be helpful for purifying FAS from pathogenic species where it is difficult to cultivate cells in bulk. 


\section{Nanobodies for restricting structural flexibility}

Preliminary tests regarding effects of nanobody binding on the conformational landscape of FAS suggested that the clone binding on the MPT domain stabilized the rotated state whereas the one bound on the AT domain had no effect. However, the nanobodies did not have conformational specificity. This could be due to the fact that the domain shift as rigid bodies between the two FAS conformations and the binding regions of the nanobodies do not alter significantly. Why, then, was there a change in the ratio of rotates/non-rotated state in the presence of $\mathrm{Nb2}$ ? A plausible explanation for this observation could be that the carbon support foil used for grid preparation interacts with the bound nanobodies which in turn affects the FAS conformation This can be tested by preparing the cryo-EM sample in the absence of any support foil and is also necessary before proceeding with future efforts on studying the effects of nanobody binding to the FAS.

Another interesting candidate is $\mathrm{Nb5}$, which binds to the structural hot-dog fold of the dehydratase domain. This nanobody is located in the proximity of the MPT domain in a manner that it would hinder the outward rotation of this domain seen in the rotated conformation of the FAS (Figure 34(ii)). If this is indeed the case then this clone can be used for assessing the role and impact of the MPT rotation on FAS activity. Characterizing the nanobodies which inhibit the fungal FAS or reduce its activity could also have great impact as anti-fungal therapeutics. 


\section{Discussion}

Large macromolecular complexes perform essential functions in the cell. These complexes are composed of smaller building blocks that together form intricate 3D assemblies. While performing their respective tasks, macromolecular complexes can adopt multiple conformations. Such conformational flexibility is functionally important but hinders our ability to study these structures at high resolution. The crucial problem of stabilizing distinct functional states of large macromolecules is a common phenomenon and is currently the major limiting factor for achieving high resolution structures. In this doctoral thesis, I studied the structural dynamics of the large multi-subunit fungal FAS. To this end, various aspects of sample preparation were tested, which resulted in resolving two distinct FAS conformations at high resolution. Furthermore, a novel $\gamma$-subunit of the yeast FAS was discovered and was found to affect the FAS activity as well as structure. The findings of this thesis and their implications on structural investigations of large molecular complexes in general, and of FAS in particular, will be the main focus of the discussion below.

\subsection{Obtaining stable macromolecular complexes for structural studies}

Various methods have been developed to tackle the two core problems of sample preparation for high resolution structural determination - compositional and conformational heterogeneity (section 1.4). Tools relevant for large macromolecular complexes such as the FAS were applied in this thesis to obtain better resolved structures of the FAS. These efforts helped in improving the resolution of the FAS structure as well as in resolving multiple conformations of this macromolecular complex. The technical aspects regarding sample preparation presented in this thesis for the FAS will be beneficial and applicable for future studies of the FAS as well as other multifunctional protein complexes. 


\subsubsection{Purification of compositionally homogeneous proteins}

The prerequisite for studying large protein complexes is to obtain intact preparations, where all the components of the complex are present in stoichiometric amounts. Failure to do so leads to heterogeneity in the sample, which hinders structure determination. Purification methods employed at the beginning of any structural study therefore need to be carefully selected. In this thesis, a chromatography-free purification under low salt conditions led to the co-purification of an accessory $\gamma$-subunit of S. cerevisiae FAS. Further, the interaction between the $\gamma$-subunit and FAS were found to be salt labile, causing the $\gamma$ subunit to dissociate at high salt concentrations of $250 \mathrm{mM} \mathrm{KCl}$. Therefore, the low salt conditions used during the chromatography-free purification were essential in preventing dissociation of the $\gamma$-subunit from the FAS during purification. Previous studies of the FAS employed methods such as anion exchange chromatography during purification. The high salt concentrations $(\sim 0.2-1 \mathrm{M})$ used in these methods could have led to at least a partial dissociation of the $\gamma$-subunit from the FAS. However, it is difficult to speculate the exact reason why the $\gamma$-subunit has remained unidentified until now.

The identification of the $\gamma$-subunit also helped in preparation of FAS as two compositionally homogenous samples - FAS without the $\gamma$-subunit and as FAS holoenzyme complex. Using these samples, it was possible to improve the resolution of FAS structures to 2.8-2.9 $\AA$ as compared to the $3.1 \AA$ structures published previously (Jenni et al., 2007; Leibundgut et al., 2007).

\subsubsection{Thermodynamic trapping of protein complexes}

\subsubsection{Working at $4^{\circ} \mathrm{C}$ to populate energetic conformational minima}

Samples for cryo-EM were prepared at $4{ }^{\circ} \mathrm{C}$. This was based on previous observations made in the lab, where lower temperatures result in trapping protein complexes, such as the ribosome, into distinct conformational minima (Fischer et al., 2010). Working at such low temperatures was indeed beneficial for structure elucidation, however, it is not the most optimal temperature for studying conformational landscapes. To improve upon this, future 
conformational analysis of FAS and similar complexes should be also performed at more physiological temperatures to access conformational states that might be unfavorable in the present experimental setup.

\subsubsection{Thermophilic vs mesophilic organisms as a source for protein complexes}

Are thermophilic variants of protein complexes more conformationally stable than those from mesophilic organisms? The answer for many large complexes including the FAS is probably "no", as there seems to be little correlation between thermal stability and conformational stability. For the FAS, cryo-EM data for both CtFAS and ScFAS displayed similar amount of conformational heterogeneity during the 3D sorting steps. Along with this, the number of particles required to obtain resolutions of $3.2 \AA$ were also found to be similar if not better for ScFAS. Identical trends can also be observed from structures of large multidomain complexes solved from both thermophilic and mesophilic sources. For example, $20 \mathrm{~S}$ proteasomes from human, yeast as well as Thermoplasma acidophilum have been resolved to resolutions of $\sim 1.8-1.9 \AA$ (Förster et al., 2005; Groll et al., 1997; Schrader et al., 2016). Therefore, improving the biochemical preparations of large protein complexes, as done for the FAS during this thesis, might be more beneficial for high resolution structural studies instead of just utilizing protein complexes from thermophilic organisms.

\subsubsection{Using protein function for conformational stabilization}

Multifunctional fatty acid synthases can have multiple intermediates bound to them, from acetyl or malonyl moieties of the substrates to intermediate acyl chains. By addition of malonyl-CoA and NADPH during purification, referred to as "cycling", all the bound intermediates are elongated to the final FAS product C16/18 acyl chain. The full length acyl chains are in turn, transferred from the ACP domain onto a free CoA molecule by the MPT domain. With this, all FAS molecules were brought to a common functional state where all the domains were empty. By doing so, the ACP domains of the FAS were found to be stabilized at the KS domain in the non-rotated conformation. In contrast, the non-rotated state of the FAS without "cycling" did not allow the visualization the ACP domains. The exact reason for this is poorly understood. However, it can be speculated to be a result of 
heterogeneity in the moieties bound to the ACP domain, which in turn affects the localization of the ACP domain. Building on these observations, unravelling the structures of the FAS with ACP stalled at one of the other four catalytic domains would require to start from an empty ACP and then develop strategies to either stop the cycle at a particular step or by loading the same intermediate analogs onto the empty ACP to force it to stall at a given active site.

\subsubsection{Using GraFix based chemical fixation}

GraFix has been previously applied for stabilizing multi-subunit complexes such as the $26 \mathrm{~S}$ proteasome and spliceosomes for cryo-EM studies (Bertram et al., 2017; Haselbach et al., 2017). For the FAS, GraFix did not affect the conformational variability of the protein but was very useful in improving the distribution of particle orientations. A uniform orientation distribution is important for cryo-EM as this method relies on $2 \mathrm{D}$ views of multiple orientations of a protein to be able to reconstruct its 3D shape. The effect of such dramatic changes in particle orientation is not reflected in the $3 \mathrm{D}$ reconstructions of the FAS due to compensation of missing particle orientations by the applied D3 symmetry. Asymmetric protein with similar orientations as the un-treated FAS would have a severe view bias causing densities to smears in the direction of the major view. This method can therefore be applied to asymmetric complexes, which suffer from such orientation bias.

\subsubsection{Nanobody based stabilization}

Recently, nanobodies have been applied for the conformational selection/stabilization of membrane proteins as well as small proteins for structural and functional studies (Dmitriev et al., 2016; Duhoo et al., 2017; Pleiner et al., 2015; Steyaert and Kobilka, 2011). Preliminary results obtained during this thesis suggest that they can be further applied for structural probing of large multidomain complexes as well. For example, one of the anti-FAS nanobody clones, $\mathrm{Nb5}$, was found to bind on the surface of the FAS, at the DH domain. The bound Nb5 was located adjacent to the MPT domain and was found to stabilize the ACPMPT domain interactions. The location of Nb5 on the FAS also suggest that it stabilizes the 
MPT-ACP domain interaction by restricting the movement of the MPT domain. Even though the extent to which Nb5 restricts the MPT domain need to be determined, such nanobodies could be an important tool in the future for understanding how dynamics of individual domains like the MPT domain affect FAS activity.

\subsection{Structural methods for studying large multidomain complexes}

\subsubsection{Studying protein structure in solution or in a crystalline lattice?}

Previous structural studies of the FAS using X-ray crystallography (Leibundgut et al., 2007) and cryo-EM (Gipson et al., 2010) have provided a conflicting structural view. The structural difference was attributed to the protein being in solution in one technique and being in a crystalline lattice in the other (Gipson et al., 2010). To make conclusive interpretation of the obtained structural data, FAS structures during this thesis were solved using both techniques mentioned above. Interestingly, both the structural states observed before were found to exist irrespective of the technique used. The structural models obtained as a result were also consistent between cryo-EM and X-ray crystallographic data. Based on these results, the method for structure determination appears to be irrelevant. Therefore, the decision about the technique to use for studying such complexes should solely be based on the quality of the data that can be obtained.

\subsubsection{Cryo-EM maps vs crystallographic maps for model building}

The crystallographic $2.9 \AA$ map of the FAS was much more detailed in comparison to the cryo-EM map at the same resolution. Densities corresponding to carbonyl bumps in the protein back bone density as well as some solvent molecules could be clearly distinguished in the X-ray map but were not as apparent in the cryo-EM map (Figure 36). In the end, model building and refinement was found to be more intuitive and resulted in better models using the X-ray map. The FAS data suggests that with the present tools, X-ray maps fare better than EM maps for building atomic models at resolutions of $\sim 3 \AA$. 
X-ray crystallography map @ 2.9 A

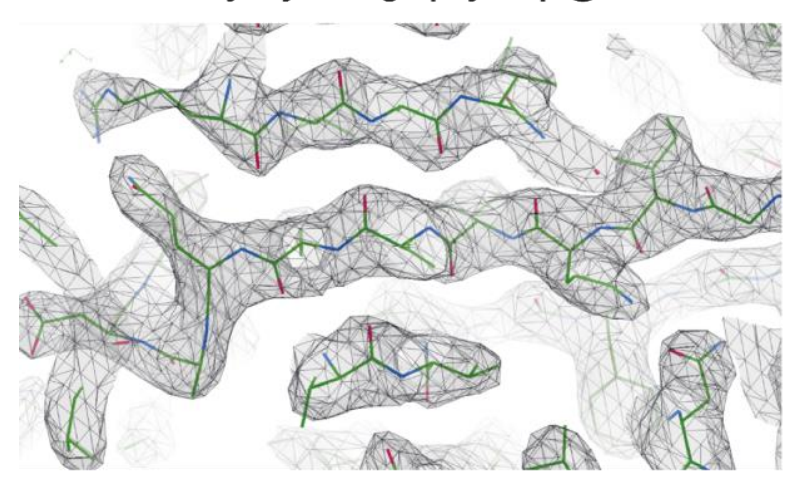

Cryo-EM map @ 2.9 A

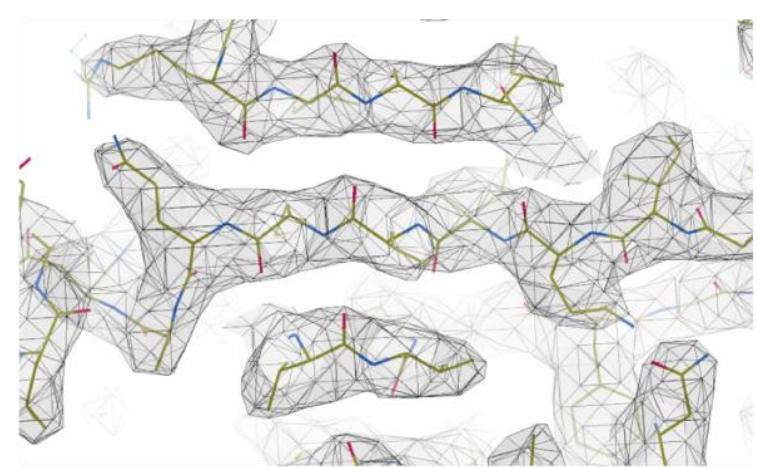

Figure 36. Comparison between FAS maps derived from X-ray crystallography and cryoEM at a resolution of 2.9 A. The crystallographic map (left) in comparison to the cryo-EM map (right) displays better defined side chain densities as well as distinct bumps corresponding to the carbonyl groups of the protein backbone.

The reasons for such differences between the maps obtained from these two structural techniques is unclear. In the context of the FAS, such differences in the map quality could arise due to the following reasons:

1) Inaccuracy in particle alignment: cryo-EM micrographs have a low signal-tonoise ratio (SNR) due to the low electron dose used for imaging. The low SNR hinders accurate particle alignment which in turn hamper resolving fine details such as the carbonyl bumps. In this thesis, Falcon III camera, in integrating-mode, was used to acquire the micrographs for determining the high resolution FAS structures. In this mode of the camera, the electrons are detected in the form of the total charge deposited on the detector. This mode of detection however leads to low SNR due to multiple electrons coinciding at the detector simultaneously. This can be improved by using the counting mode of the Falcon III where the imaging is done in a manner that the camera can detect each incident electron. The SNR in the counting-mode at high resolution is reported to be almost twice as much as in the integrating-mode. This could be tested for the FAS in the future to examine whether data acquisition in counting-mode would improve upon the quality of the present cryo-EM maps of the FAS.

2) D3-symmetry applied during 3D refinement: the fungal type I FAS has an apparent D3-symmetry due to which a D3-symmetry was applied during the 3D refinement procedures. Since, the crystallographic FAS model and FAS model from the D3-symmetric 
cryo-EM map were nearly identical, the symmetry applied does not seem to introduce a perceivable artifacts in the FAS structure. However, it cannot be ruled out that deviations from the D3-symmetry are not present in the particle used for 3D refinement. It is possible that minor deviations in symmetry lead to averaging of fine details in the resulting structure. The deviation of one or more FAS subunits from the applied D3-symmetry would affect the accuracy of particle alignment. Image processing of the cryo-EM data for the FAS without applying symmetries would be of great interest and might help understand the limitations that applying symmetries can have on the FAS structure.

\subsubsection{D PCA - energy landscapes as a tool for analyzing cryo-EM data}

Cryo-EM image processing involves multiple 3D sorting steps where many less abundant conformations and compositionally heterogeneous particles are sorted out. This results in the structural determination of only one or a few possible conformational states at high resolution, despite the fact that many other conformations may also exist in the sample. To complement these structural analyses, tools like 3D PCA that generate energy landscapes can help analyze the structural states of all the particles present in a dataset. Such analysis can provide valuable information regarding (1) the number of energetic minima in a dataset, (2) major modes of motion within a complex and (3) subunits or factors that have variable occupancy(Haselbach et al., 2017). Since this analysis can be performed at resolutions of $\sim 20 \AA$, it can always be done with a small subset of particles to estimate the number of conformations one might expect or the regions of the complex that are stable or dynamic. As shown with the $\gamma$-subunit, energy landscapes can also be used to visualize the effect of protein-protein interactions on conformational landscapes. Finally, since there is a lot of interest in using cryo-EM for high throughput screening, one could in principle apply this method for this purpose as well. For example, in the case of the FAS, since we have a reference energy landscape, small datasets of $~ 200-300$ micrographs at lower magnifications to obtain $\sim 100,000$ particles should be enough to detect whether a small molecule or protein stabilizes a particular FAS conformation using this method. In general, such a tool is complementary to the present cryo-EM image processing pipelines and provides a possibility to analyze the low-resolution information present in the structural data. 


\subsection{Novel insights into fungal FAS machinery}

\subsubsection{Conformations of the FAS dome correlate with ACP movement}

Structural studies of FAS have postulated that this complex has a static two barrel chamber with a dynamic acyl carrier protein inside, which carries substrates and elongating intermediates from one active site to another in a stochastic manner during fatty acid synthesis (Anselmi et al., 2010; Gipson et al., 2010; Jenni et al., 2007). Insights gained in this study using X-ray crystallography and cryo-EM provide an alternate view of how this machinery functions. In the absence of bound substrates or intermediates, the FAS dome can adopt two conformations - a rotated one with the ACP stalled at the AT domain and a non-rotated one, where the ACP is stalled at the KS domain. The specificity of the ACP for the AT domain in the rotated state can be explained by the increase in the binding surface area created by the downward shift of the AT domain

Functionally, the rotated state would correspond to the structure of the FAS at the first step of fatty acid synthesis, where the acetyl group is loaded onto the phosphopantetheine arm of the ACP domain. In contrast, the non-rotated state with the ACP domain at the KS site would correspond to the step where an acetyl/malonyl group or an intermediate acyl chain is shuttled to the KS active site. Therefore, the present structures can be viewed as snapshots of two steps in the fatty acid synthesis cycle.

In general, these findings add to our understanding of the ACP shuttling process. The correlation between ACP position and the state of the FAS dome was never observed before or considered when computational models for ACP shuttling were generated, making it necessary to update previous studies based on these observations.

\subsubsection{Conformational changes provide directionality to ACP movement}

The conformational changes in the rotated state of the FAS strongly affects two catalytic domains - AT and MPT. Upon rotation of the $\beta$-subunit, the ACP interacts with the AT domain in a stable manner. Concurrently, the MPT domain moves outwards with respect of the FAS dome. It is also possible that such an outward facing conformation of the MPT 
domain makes this site less favorable for interactions with the ACP domain. However, more structural evidence is required to make a conclusive statement regarding the dependence of ACP domain binding on the MPT conformation. Nevertheless, structural changes of the FAS dome could direct the movement of the ACP, at least towards the AT domain during the catalytic cycle. This ability of the FAS to direct the movement of the ACP towards one of its domains suggests at the possibility that the movement of ACP inside the FAS barrel could be guided by structural changes in the $\beta$-subunits. From the data presented in this thesis, this appears to be likely for the first step of the fatty acid synthesis cycle where an acetyl group is transferred to the ACP domain. The remaining domains of the FAS dome - namely, KS, KR, ER and DH - remain relatively unchanged between the rotated and non-rotated FAS conformation. Therefore, at present, it is difficult to predict without further structural evidence whether the $\mathrm{ACP}$ binding to these four domains is preferred in a particular FAS conformation.

These findings indicate that the shuttling of substrates might be a more ordered phenomenon than previously suggested (Anselmi et al., 2010; Jenni et al., 2007; Leibundgut et al., 2007). The notion of ACP shuttling as a stochastic process is now uncertain in light of the directionality provided to the ACP by rotation of the FAS dome. FAS structures with the ACP stalled at the different domains of the FAS are therefore key for solving this puzzle and for obtaining a definitive model for substrate shuttling by the ACP.

\subsubsection{Tma17p - a novel $\gamma$-subunit of the yeast FAS}

Along with insights into the dynamics of the FAS structure, the sample preparation methods used during the course of this study led to the discovery of a novel $\gamma$-subunit of the yeast FAS. A protein previously termed as Tma17 (translational machinery associated protein) (Fleischer et al., 2006)/Adc17 (ATPase dedicated chaperone) (Hanssum et al., 2014) was found to be bound to FAS purified from S. cerevisiae. Yeast deletion strains, reconstitution assays, crosslinking mass spectrometry along with high resolution structures not only confirmed this initial observation, but also showed that Tma17p is a specific interacting partner of the FAS. It is thus quite surprising that there were no previous reports regarding a "Tma17p" with the ability to bind the fungal FAS. However, Tma17p-FAS 
complex has been detected in two previous publications before, but was apparently not characterized. Retrospective analysis of data from these publications have brought forward the following observations:

1) The initial identification of the Tma17p as a translational machinery associated protein by Fleischer et al., (2006) were based on its co-sedimentation with ribosomal proteins on sucrose gradients. Mass spectrometry analysis used in their study revealed that Tma17 was present in fractions containing $40 S$ and 605 subunits. Interestingly, so was the FAS. In a reverse experiment, TAP-tagged Tma17 was used to pull down associated proteins. No ribosomal proteins were found bound to it, which is why this study did not investigate this protein any further. However the most abundant protein bound to Tma17 was the FAS. Fleischer et al., (2006) never claimed that Tma17 was associated with the ribosomes, however, their results have been misinterpreted leading to the characterization of Tma17 as a ribosome associated protein in all public databases like UniProt and yeastgenome.org.

2) The cryo-EM structure of the yeast FAS by Gipson et al., (2010) was the first sub $6 \AA$ Atructure solved of this complex by this method. This study found the FAS dome to adopt a rotated conformation. Additionally, two unknown, long cylindrical densities were observed. They misinterpreted these as two locations for the ACP linker regions. In light of the findings of this thesis, we now know that these densities can be attributed to the $\gamma$ subunit and that the FAS dome conformation was stabilized in the rotated state because of the bound $\gamma$-subunit. This example illustrates that cryo-EM densities at low-resolutions should be carefully evaluated. Additional methods such as cross-linking mass spectrometry should be routinely employed for such structural data in order to obtain more reliable conclusions.

Thus, the interaction between FAS and the $\gamma$-subunit indicated by pull-down assays, mass spectrometry along with structural data showing stabilization of the rotated FAS conformation by the $\gamma$-subunit were already present. However, due to limitations of using only single techniques, the interaction between the $\gamma$-subunit and FAS was overlooked or misinterpreted. In this thesis, different approaches such as XL-MS, kinetic and structural 
analysis allowed the identification and characterization of the Tma17p as the $\gamma$-subunit of the yeast FAS.

\subsubsection{Is the $\gamma$-subunit involved in different cellular processes?}

Apart from interacting with the FAS, the $\gamma$-subunit has been interpreted to be an ATPase dedicated chaperone. Based on their biochemical evidence, Hanssum et al. ( 2014) interpreted that the $\gamma$-subunit interacts with Rpt6 subunit of the $26 \mathrm{~S}$ proteasome and assists in the assembly of the complex. However, there are some discrepancies within the data presented in this paper. (1) Immunoprecipitation of Flag-tagged $\gamma$-subunit led to the identification of only Rpt6 as its interactor. This contradicts the observations of Fleischer et al. (2006) who reported FAS as the most abundant $\gamma$-subunit bound species along with insignificant amounts of Rpt6. (2) Using yeast two-hybrid assays, binding of the $\gamma$-subunit with full length Rpt6 was found to be very weak and only the N-terminal coiled coil domain of Rpt6 (NT-Rpt6) was found to strongly interact with the $\gamma$-subunit. The differences in the ability of the $\gamma$-subunit to bind the full length and truncated version of Rpt6 was, however, not investigated. Further, they did not perform binding assays to determine in vitro binding affinity of the $\gamma$-subunit to Rpt6. This raises the question of whether the interaction between the $\gamma$-subunit and Rpt6 is indeed specific. (3) Formation of a NT-Rpt6 and NT-Rpt3 complex upon co-expression with the $\gamma$-subunit in E. coli was then used as a functional assay to show the $\gamma$-subunit chaperone activity. Since all the biochemical assays depicting the chaperone activity of the $\gamma$-subunit were performed using the N-terminal segment of Rpt6 and Rpt3, it is again difficult to conclude if the results obtained were due to specific or non-specific interactions. (4) Growth defects in the $\gamma$-subunit deletion strains could not be rescued by overexpressing other Rpt6 chaperones indicating that $\gamma$-subunit dependent growth defects could arise because of reasons other than proteasome assembly. (5) In WT cells, there was no correlation between the presence/absence of the $\gamma$-subunit and cellular levels of proteasome subunits or $26 \mathrm{~S}$ proteasomes. (6) Finally, the correlation between an increase in cellular stress and $\gamma$-subunit expression was also shown. However, the relationship between increased $\gamma$-subunit expression under stress and its effect on proteasome assembly is inconclusive. For example, in wild type cells, proteasome subunit levels, including Rpt6, 
were unaffected by the presence or absence of the $\gamma$-subunit. This observation contradicts the experiments where the authors show a decrease in Rpt6 levels when proteasome assembly is affected. In addition, the reduction in 305 proteasome levels and activity in the absence of the $\gamma$-subunit are statistically on the borderline of being considered significant (p-value of 0.05). Taken together, these points indicate that further investigations are necessary to distinguish whether the effects on yeast cells in the absence of the $\gamma$-subunit are due to its role in modulating FAS activity or proteasome assembly, or perhaps both.

\subsubsection{Present hypothesis for the role of $\gamma$-subunit in the cell}

Prior to this thesis, along with the mischaracterization of the $\gamma$-subunit as a ribosome associated protein, it was also reported to have an increased cellular expression during stress and act as a chaperone for $26 \mathrm{~S}$ proteasome assembly (Fleischer et al., 2006; Hanssum et al., 2014; Murphy et al., 2015; Rousseau and Bertolotti, 2016). Based on these publication and the data presented in this thesis, I propose the following hypothesis for the role of $\gamma$ subunit in the cell:

Upon stress conditions like heat shock, glucose depletion or treatment with tunicamycin or rapamycin, cellular $\gamma$-subunit levels increase manifold. This increase in protein expression is not due to the unfolded protein response and is mediated by Map kinase1 in Hog1 or TORC1-dependent manner. This pathway is also responsible for increase in the levels of proteasome subunits and assembly chaperones to cope with the stress. The $\gamma$-subunit can then performs two functions in the cell:

1) The $\gamma$-subunit binds to the FAS and decreases its activity to prevent futile consumption of energy rich substrates such as acetyl-CoA, ATP and NADPH for fatty acid synthesis. Since the reduction in FAS activity is essential for cell viability under stress conditions (Shpilka et al., 2015), the $\gamma$-subunit can function as the protein responsible for regulating FAS activity. Therefore, as cells detect stressful conditions, increase in $\gamma$-subunit concentrations could be used as immediate short term regulation of FAS which can further be degraded if the stress conditions persist. 
2) The $\gamma$-subunit could act as a chaperone for assembling $26 \mathrm{~S}$ proteasome through its interactions with the coiled coil domain of Rpt6 under stress conditions to cope with the increased amounts of ubiquitinated proteins. However, this cellular function of the $\gamma$ subunit needs further experimental evidence due to reasons described in section 4.3.4.

Since the $\gamma$-subunit is also present in stress free conditions, it may also be used to ensure that FAS uses its substrates only when they are present above a certain threshold concentration. This would be helpful for the cell as the substrate binding affinity of FAS is very high in absence of the $\gamma$-subunit, in the range of $\sim 10 \mu \mathrm{M}$. The cell can therefore manipulate the shuttling of metabolites into fatty acid synthesis by modulating $\gamma$-subunit concentration even in a stress-free state.

Therefore, the $\gamma$-subunit can have potentially two cellular roles: (1) as a post-translational regulator of FAS through which the cell can precisely control when and how much fatty acids to synthesize, in both physiological and stress conditions and (2) as a chaperone for $26 \mathrm{~S}$ proteasome assembly during stress conditions.

\subsection{4. $\gamma$-subunit is conserved among yeast but not in higher eukaryotes}

The binding mechanism of the $\gamma$-subunit makes it optimal for binding only the $\alpha_{6}, \beta_{6}$ fungal type I FAS. In comparison, structures of type II FAS as well as metazoan type I FAS have distinctly different architectures and would need a protein with a different structure than the $\gamma$-subunit to perform similar functions. As expected, sequence conservation based searches resulted in no hits outside the fungi kingdom. Interestingly, even within fungi, the $\gamma$-subunit is mostly conserved in the phylum of Ascomycota. Sequence identity ranged from $30-95 \%$ whereas the overall protein length varied from 140-170 amino acids. Other fungal species either might have another protein analogous to the $\gamma$-subunit but with a different structure or they might regulate FAS activity through other unknown mechanisms. 


\subsubsection{Biotechnological applications of the FAS and its $\gamma$-subunit}

Using microbes for production of fatty acid or fatty acid derived products (known as oleochemicals) offers a cheap and sustainable alternative to vegetable oils and animal fats as source of raw materials for industrial production of commodities such as biodiesel, detergents, lubricants, bioplastics, emulsifiers, coatings, food and feed additives (Janßen and Steinbüchel, 2014; Peralta-Yahya et al., 2012). In particular, S. cerevisae has been engineered to produce fuels like ethanol, isobutanol, butanol, and farnesene (Zhou et al., 2014). Further ways to increase yields as well as diversify the products are being investigated. Structural information of enzymes involved in fatty acids synthesis aid such endeavours, as they allow for targetted manipulation of the FAS. For example, multiple mutations in the fungal FAS based on structural data were screened to produce short chain fatty acids (SCFAs) using S. cerevisiae FAS or polyketides using C. ammoniagenes FAS (Gajewski et al., 2017a, 2017b). In particular, for the production of SCFAs, out of the different combinations of mutations in AT (I306S), MPT (R1834K) and KS (G1250S, M1251W, F1279Y), it was observed that R1834K favoured C8-FA, I306A-G1250S favored C6-FA and I306A-G1250S-R1834K resulted in a mixture of C6-FA/C8-FA. The mutations were intended to affect substrate affinities at the modified active sites, however, the exact effect could only be determined experimentally. More comprehensive structural data comprising of different conformational states of FAS would help diversify the compounds synthesised by it using strategies similar to those described by Gajewski et al., (2017b).

In addition, the knowledge about the existence and mechanism of the $\gamma$-subunit in FAS regulation has many biotechnological implications. Yeast strains used for industrial production of fatty acids could be modified by genomic deletion of the $\gamma$-subunit, which might help in achieving higher output due to the absence of a protein that reduces the affinity of FAS for its substrates. Since the $\gamma$-subunit binds inside the FAS barrel, it can be used to localize new enzymatic modules to the FAS. This would allow for the diversification of the compounds produced by this machinery. It is known that a $20 \mathrm{kDa}$ TAP-tag does not hinder the binding of the $\gamma$-subunit to FAS (Fleischer et al., 2006), however, the limit for the size and number of the additional proteins that can be tethered to it would need to be tested. Finally, the C-terminal residues that bind to the KR active site cleft offer a scaffold for 
designing potent inhibitors specific for the fungal FAS. The binding affinity of this peptide fragment itself could also be further optimized using random mutation and screening approaches to create high affinity inhibitors specific for different types of NADPH-binding enzymes. 


\section{Conclusion and Outlook}

In this doctoral thesis, sample preparation methods to tackle problems of compositional and conformational heterogeneity of the type I fungal fatty acid synthase were tested. The type I fungal FAS used as a model system helped shed light on the impact of proper biochemical handling of large complexes on the quality of the structural data that is obtained from them.

In contrast to previously employed purification strategies for the type I fungal FAS, a chromatography-free purification was found to be beneficial. This is illustrated by the copurification of a novel $\gamma$-subunit of the FAS. These biochemical preparations of the FAS helped in advancing the quality of structural data by allowing for reproducibly achieving resolutions of $\sim 3.0 \AA$ A using both X-ray crystallography and cryo-EM. Different biochemical treatments were then tested to further improve the resolution of the FAS structure. GraFix was shown to be effective in overcoming orientation bias that macromolecules might have on an EM grid but did not affect the resolution of the structure. On the other hand, "cycling" of the FAS with its substrates was crucial in stabilizing the otherwise dynamic ACP domain. This helped in improving the resolution by $0.3-0.4 \AA$ to $\sim 2.8 \AA$. Along with this, anti-FAS nanobodies were found to be promising tools for conformational stabilization of large macromolecular complexes such as the FAS.

The good quality of sample preparations and structural data that could be obtained using them allowed for gaining novel insights into the fungal type I FAS. FAS was found to have two distinct conformations - a rotated one with the ACP domain stalled at the AT domain and a non-rotated one where the ACP domain is stalled at the KS domain. These observations indicate at a non-stochastic model for ACP shuttling, where the FAS can modulate ACP movement using its dome conformation. In addition, a novel $\gamma$-subunit was found to co-purify along with the FAS. With the help of kinetic analysis, XL-MS and highresolution structures, the molecular basis underlying the ability of the $\gamma$-subunit to affect FAS activity and stabilize its rotated conformation was also deciphered. The $\gamma$-subunit spans a distance of $120 \AA$ inside the FAS dome while interacting with the ER, ACP, MPT and KR domains. The $\mathrm{N}$ - and C-terminal segments of the $\gamma$-subunit sterically compete with 
NADPH binding to the ER and KR domain, whereas, its negatively charged aspartate rich loop segment present near the MPT domain hinders malonyl-CoA binding to the MPT domain. Through these interactions, the $\gamma$-subunit stabilizes the rotated conformation of the FAS as well as reduces the affinity of FAS towards its substrates.

These observations open the following avenues for future investigations:

\section{1) Applying the knowledge gained regarding sample preparation to other large macromolecular complexes.}

The concepts for purification and biochemical treatments applied in this thesis could be applied for complexes such as the mammalian FAS or polyketide synthases. These proteins have a similar modular arrangement and use similar mechanisms for substrate shuttling and catalysis as the fungal FAS (Herbst et al., 2018). If found helpful, these principles can then be used for more distinct and dynamic protein complexes such as oxoglutarate dehydrogenase or pyruvate dehydrogenase.

\section{2) Understanding the structural basis behind ACP shuttling.}

To build on the observations of directionality provided to the ACP domain by the FAS conformation, further snapshots of the FAS with ACP domain bound at the KR, DH ER and MPT domains are necessary. For achieving this, the phosphopantetheine arm of the ACP domains need to be modified with analogs for acyl chain intermediates. The use of nanobodies for this purpose should also be tested as preliminary tests with Nb5 show promising results in this direction.

\section{3) Determining the role of the $\gamma$-subunit in the cell}

The model proposed for the role of the $\gamma$-subunit in vivo needs to be verified using cellular studies for conclusive interpretation of all data that is available for this protein. This would also helpful in avoiding possible misinterpretation of biochemical data from future studies of the FAS and proteasome assembly. 


\section{Abbreviations}

2D

3D

ACP

ADP

AT

ATP

BS3

BSA

CoA

cryo-EM

CTF

CtFAS Chaetomium thermophilum fatty acid synthase

DH dehydratase

DMSO dimethyl sulfoxide

DNA deoxyribonucleic acid

DTT dithiothreitol

EDC 1-ethyl-3-(3-dimethylaminopropyl)carbodiimide hydrochloride

EDTA ethylenediaminetetraacetic acid

EMDB Electron Microscopy Data Bank

ER enoylreductase

FAS fatty acid synthase

FSC Fourier shell correlation

HEPES 4-(2-hydroxyethyl)-1-piperazineethanesulfonic acid

kDa kilo Dalton

KR ketoreductase

LMNG lauryl maltose neopentyl glycol

MES 2-(N-morpholino)ethanesulfonic acid

MPT malonyl/palmitoyl transferase 
MW molecular weight

NADH Nicotinamide adenine dinucleotide

NADPH Nicotinamide adenine dinucleotide phosphate

OGNG octyl glucose neopentyl glycol

PCA principle component analysis

PDB Protein Data Bank

PEG polyethylene glycol

PMSF phenylmethane sulfonyl fluoride

PPT phosphopantetheine transferase

RNA ribonucleic acid

ScFAS Sacharomyces cerevisiae fatty acid synthase

SDA succinimidyl 4,4'-azipentanoate

SDS-PAGE sodium dodecyl sulfate polyacrylamide gel electrophoresis

TRIS tris(hydroxymethyl)aminomethane 


\section{Supplementary Information}

\section{A. Supplementary tables}

Table 1. Tandem Mass Spectrometry analysis of the purified FAS sample in Figure 10(ii).

\begin{tabular}{|c|c|c|c|}
\hline Protein Identified & $\begin{array}{l}\text { Accession } \\
\text { Number }\end{array}$ & $\begin{array}{c}\text { Molecular } \\
\text { Weight }\end{array}$ & $\begin{array}{c}\text { No. of } \\
\text { Peptides }\end{array}$ \\
\hline Fatty acid synthase alpha & FAS2_YEAST & $207 \mathrm{kDa}$ & 177 \\
\hline Fatty acid synthase beta & FAS1_YEAST & $229 \mathrm{kDa}$ & 162 \\
\hline Translation machinery-associated protein 17 & TMA17_YEAST & $17 \mathrm{kDa}$ & 114 \\
\hline Glucokinase-1 & HXKG_YEAST & $55 \mathrm{kDa}$ & 33 \\
\hline Probable E3 ubiquitin-protein ligase TOM1 & TOM1_ASHGO & $372 \mathrm{kDa}$ & 20 \\
\hline Eukaryotic translation initiation factor $1 \mathrm{~A}$ & IF1A_YEAST & $17 \mathrm{kDa}$ & 14 \\
\hline Proteasome subunit beta type- 5 & PSB5_YEAST & $32 \mathrm{kDa}$ & 12 \\
\hline Eukaryotic translation initiation factor $5 \mathrm{~A}-1$ & IF5A1_YEAST & $17 \mathrm{kDa}$ & 11 \\
\hline Proteasome subunit beta type- 1 & PSB1_YEAST & $24 \mathrm{kDa}$ & 9 \\
\hline Proteasome subunit beta type- 3 & PSB3_YEAST & $23 \mathrm{kDa}$ & 7 \\
\hline Cap-associated protein CAF20 & CAF20_YEAST & $18 \mathrm{kDa}$ & 6 \\
\hline Altered inheritance of mitochondria protein 41 & AIM41_YEAST & $21 \mathrm{kDa}$ & 6 \\
\hline Proteasome subunit beta type- 2 & PSB2_YEAST & $28 \mathrm{kDa}$ & 6 \\
\hline ADP-ribosylation factor 1 & ARF1_YEAST & $21 \mathrm{kDa}$ & 5 \\
\hline Proteasome subunit beta type- 4 & PSB4_YEAST & $23 \mathrm{kDa}$ & 5 \\
\hline $40 S$ ribosomal protein S11-A & RS11A_YEAST & $18 \mathrm{kDa}$ & 3 \\
\hline Protein transport protein SFT2 & SFT2_YEAST & $24 \mathrm{kDa}$ & 2 \\
\hline
\end{tabular}


Table 2. XL-MS analysis of endogenous $\gamma$-subunit-FAS complex.

Statistics (Crosslinked peptide spectrum matches (CSMs) and Scoremax) of the Crosslinking-MS data for the endogenous $\gamma$-subunit -FAS complex from S. cerevisiae. "Inter"- protein crosslinks between FAS and $\gamma$-subunit where numbers in the Residue 1 and 2 columns indicate the position of the cross-linked residue.

\begin{tabular}{|c|c|c|c|c|c|c|c|c|}
\hline Type & Protein 1 & $\begin{array}{c}\text { UniProt } \\
\text { AC } 1\end{array}$ & Protein 2 & $\begin{array}{c}\text { UniProt } \\
\text { AC } 2 \\
\end{array}$ & $\begin{array}{c}\text { Residue } \\
1 \\
\end{array}$ & $\begin{array}{c}\text { Residue } \\
2 \\
\end{array}$ & CSM & $\begin{array}{c}\begin{array}{c}\text { Best Score } \\
(\max )\end{array} \\
\end{array}$ \\
\hline \multirow[t]{14}{*}{ BS3 } & \multirow{11}{*}{$\begin{array}{c}\text { FAS alpha- } \\
\text { subunit }\end{array}$} & \multirow[t]{11}{*}{ P19097 } & \multirow[t]{11}{*}{$\gamma$-subunit } & \multirow[t]{11}{*}{ Q12513 } & 105 & 55 & 8 & 16.35 \\
\hline & & & & & 105 & 51 & 12 & 3.85 \\
\hline & & & & & 105 & 118 & 3 & 3.73 \\
\hline & & & & & 1079 & 118 & 7 & 18.85 \\
\hline & & & & & 1081 & 111 & 47 & 16.16 \\
\hline & & & & & 1081 & 116 & 17 & 10.64 \\
\hline & & & & & 1081 & 118 & 66 & 17.28 \\
\hline & & & & & 1087 & 118 & 15 & 13.45 \\
\hline & & & & & 1092 & 116 & 28 & 6.3 \\
\hline & & & & & 1092 & 118 & 47 & 11.95 \\
\hline & & & & & 1092 & 124 & 9 & 9.32 \\
\hline & \multirow[t]{3}{*}{$\begin{array}{c}\text { FAS beta- } \\
\text { subunit }\end{array}$} & \multirow[t]{3}{*}{ P07149 } & \multirow[t]{3}{*}{$\gamma$-subunit } & \multirow[t]{3}{*}{ Q12513 } & 877 & 17 & 18 & 11.43 \\
\hline & & & & & 879 & 83 & 17 & 18.75 \\
\hline & & & & & 1031 & 17 & 34 & 31.35 \\
\hline \multicolumn{7}{|l|}{ Total } & 328 & 31.35 \\
\hline Type & Protein 1 & $\begin{array}{c}\text { UniProt } \\
\text { AC } 1\end{array}$ & Protein 2 & $\begin{array}{c}\text { UniProt } \\
\text { AC } 2\end{array}$ & $\begin{array}{c}\text { Residue } \\
1 \\
\end{array}$ & $\begin{array}{c}\text { Residue } \\
2 \\
\end{array}$ & CSM & $\begin{array}{c}\text { Best Score } \\
\text { (max) }\end{array}$ \\
\hline \multirow[t]{14}{*}{ SDA } & \multirow[t]{14}{*}{$\begin{array}{c}\text { FAS alpha- } \\
\text { subunit }\end{array}$} & \multirow[t]{14}{*}{ P19097 } & \multirow[t]{14}{*}{$\gamma$-subunit } & \multirow[t]{14}{*}{ Q12513 } & 1741 & 20 & 2 & 14.03810453 \\
\hline & & & & & 712 & 22 & 2 & 10.57675413 \\
\hline & & & & & 106 & 55 & 2 & 4.003926346 \\
\hline & & & & & 118 & 55 & 2 & 12.86012091 \\
\hline & & & & & 119 & 55 & 3 & 10.83268267 \\
\hline & & & & & 92 & 55 & 7 & 9.202040356 \\
\hline & & & & & 93 & 55 & 2 & 7.935542011 \\
\hline & & & & & 105 & 57 & 2 & 13.21681131 \\
\hline & & & & & 62 & 62 & 2 & 12.89619628 \\
\hline & & & & & 62 & 63 & 4 & 18.09312647 \\
\hline & & & & & 64 & 63 & 3 & 18.75945075 \\
\hline & & & & & 62 & 64 & 10 & 22.7619539 \\
\hline & & & & & 64 & 64 & 5 & 18.64016452 \\
\hline & & & & & 62 & 65 & 4 & 18.17263073 \\
\hline
\end{tabular}




\begin{tabular}{|c|c|c|c|c|c|c|c|}
\hline & & & & 64 & 65 & 3 & 14.88941029 \\
\hline & & & & 62 & 66 & 2 & 13.52287875 \\
\hline & & & & 62 & 68 & 3 & 27.01863449 \\
\hline & & & & 62 & 69 & 3 & 14.53610701 \\
\hline & & & & 64 & 69 & 2 & 23.05109824 \\
\hline & & & & 62 & 70 & 8 & 23.18309616 \\
\hline & & & & 64 & 70 & 2 & 13.06701918 \\
\hline & & & & 62 & 71 & 15 & 31.69250396 \\
\hline & & & & 64 & 71 & 17 & 25.30715308 \\
\hline & & & & 62 & 72 & 6 & 16.76955108 \\
\hline & & & & 64 & 72 & 3 & 17.05650548 \\
\hline & & & & 62 & 73 & 2 & 9.640164518 \\
\hline & & & & 1092 & 117 & 10 & 7.829738285 \\
\hline & & & & 1080 & 118 & 3 & 12.12205305 \\
\hline & & & & 1082 & 118 & 3 & 9.474955193 \\
\hline & & & & 1092 & 118 & 8 & 8.104025268 \\
\hline & & & & 1079 & 119 & 2 & 7.358525889 \\
\hline & & & & 1092 & 119 & 5 & 8.694648631 \\
\hline & & & & 1092 & 123 & 17 & 9.505845406 \\
\hline & & & & 1097 & 124 & 2 & 7.605548319 \\
\hline & & & & 580 & 148 & 2 & 12.95860731 \\
\hline $\begin{array}{l}\text { FAS beta- } \\
\text { subunit }\end{array}$ & P07149 & $\gamma$-subunit & Q12513 & 1031 & 15 & 14 & 9.970616222 \\
\hline & & & & 1921 & 56 & 4 & 10.66554625 \\
\hline & & & & 1768 & 63 & 2 & 21.23358715 \\
\hline & & & & 879 & 64 & 3 & 16.075204 \\
\hline & & & & 879 & 65 & 2 & 12.49485002 \\
\hline & & & & 1768 & 65 & 2 & 20.49079748 \\
\hline & & & & 1918 & 68 & 7 & 7.222573178 \\
\hline & & & & 1768 & 69 & 3 & 11.61261017 \\
\hline & & & & 879 & 70 & 2 & 10.5421181 \\
\hline & & & & 1918 & 70 & 8 & 7.222573178 \\
\hline & & & & 1921 & 70 & 3 & 6.200659451 \\
\hline & & & & 879 & 71 & 5 & 11.61978876 \\
\hline & & & & 1918 & 71 & 13 & 8.435333936 \\
\hline & & & & 1921 & 71 & 5 & 15.26042766 \\
\hline & & & & 838 & 72 & 4 & 14.47886192 \\
\hline & & & & 879 & 72 & 9 & 10.95860731 \\
\hline & & & & 1918 & 72 & 4 & 9.534617149 \\
\hline & & & & 1921 & 72 & 2 & 13.94692156 \\
\hline & & & & 1918 & 73 & 2 & 10.1414628 \\
\hline & & & & 877 & 88 & 3 & 6.548213564 \\
\hline & & & & 879 & 88 & 7 & 16.34678749 \\
\hline
\end{tabular}




\begin{tabular}{|c|c|c|c|c|c|c|c|c|}
\hline & & & & & $\begin{array}{l}877 \\
877\end{array}$ & $\begin{array}{l}97 \\
98\end{array}$ & $\begin{array}{l}2 \\
3\end{array}$ & $\begin{array}{c}12.47625353 \\
13.1837587\end{array}$ \\
\hline Total & & & & & & & 277 & 31.69250396 \\
\hline Type & Protein 1 & $\begin{array}{c}\text { UniProt } \\
\text { AC } 1\end{array}$ & Protein 2 & $\begin{array}{c}\text { UniProt } \\
\text { AC } 2\end{array}$ & $\begin{array}{c}\text { Residue } \\
1\end{array}$ & $\begin{array}{c}\text { Residue } \\
2\end{array}$ & CSM & $\begin{array}{l}\text { Best Score } \\
\text { (max) }\end{array}$ \\
\hline \multirow[t]{37}{*}{ EDC } & $\begin{array}{l}\text { FAS alpha- } \\
\text { subunit }\end{array}$ & P19097 & $\gamma$-subunit & Q12513 & 101 & 51 & 8 & 4.277366077 \\
\hline & & & & & 106 & 51 & 3 & 4.298432015 \\
\hline & & & & & 96 & 55 & 2 & 3.649751982 \\
\hline & & & & & 101 & 55 & 15 & 15.6216021 \\
\hline & & & & & 102 & 55 & 4 & 9.368556231 \\
\hline & & & & & 106 & 55 & 5 & 13.59006688 \\
\hline & & & & & 107 & 55 & 7 & 14.93930216 \\
\hline & & & & & 96 & 55 & 7 & 8.575118363 \\
\hline & & & & & 105 & 57 & 3 & 12.58670024 \\
\hline & & & & & 105 & 60 & 3 & 12.36754271 \\
\hline & & & & & 62 & 61 & 4 & 14.11350927 \\
\hline & & & & & 64 & 61 & 2 & 27.32697909 \\
\hline & & & & & 62 & 64 & 47 & 31.95467702 \\
\hline & & & & & 64 & 64 & 9 & 26.60906489 \\
\hline & & & & & 83 & 64 & 4 & 10.43415218 \\
\hline & & & & & 62 & 66 & 12 & 18.99139983 \\
\hline & & & & & 64 & 66 & 4 & 19.5421181 \\
\hline & & & & & 62 & 67 & 5 & 27.63078414 \\
\hline & & & & & 105 & 69 & 2 & 10.98296666 \\
\hline & & & & & 62 & 69 & 12 & 24.26201267 \\
\hline & & & & & 64 & 69 & 8 & 25.3439018 \\
\hline & & & & & 83 & 69 & 3 & 8.354577731 \\
\hline & & & & & 105 & 70 & 2 & 9.920818754 \\
\hline & & & & & 62 & 70 & 18 & 24.09963287 \\
\hline & & & & & 64 & 70 & 6 & 14.89619628 \\
\hline & & & & & 62 & 72 & 25 & 25.26841123 \\
\hline & & & & & 64 & 72 & 8 & 16.63451202 \\
\hline & & & & & 101 & 118 & 3 & 5.744727495 \\
\hline & & & & & 107 & 118 & 4 & 9.478861916 \\
\hline & & & & & 1109 & 118 & 9 & 11.58670024 \\
\hline & & & & & 1092 & 120 & 7 & 8.728158393 \\
\hline & & & & & 1099 & 124 & 3 & 5.186419011 \\
\hline & & & & & 1120 & 129 & 2 & 11.73282827 \\
\hline & & & & & 1128 & 129 & 2 & 9.8569852 \\
\hline & \multirow{3}{*}{$\begin{array}{l}\text { FAS beta- } \\
\text { subunit }\end{array}$} & P07149 & $\gamma$-subunit & Q12513 & 1031 & 14 & 10 & 11.25026368 \\
\hline & & & & & 1215 & 25 & 4 & 7.251037139 \\
\hline & & & & & 1106 & 55 & 5 & 23.4710833 \\
\hline
\end{tabular}




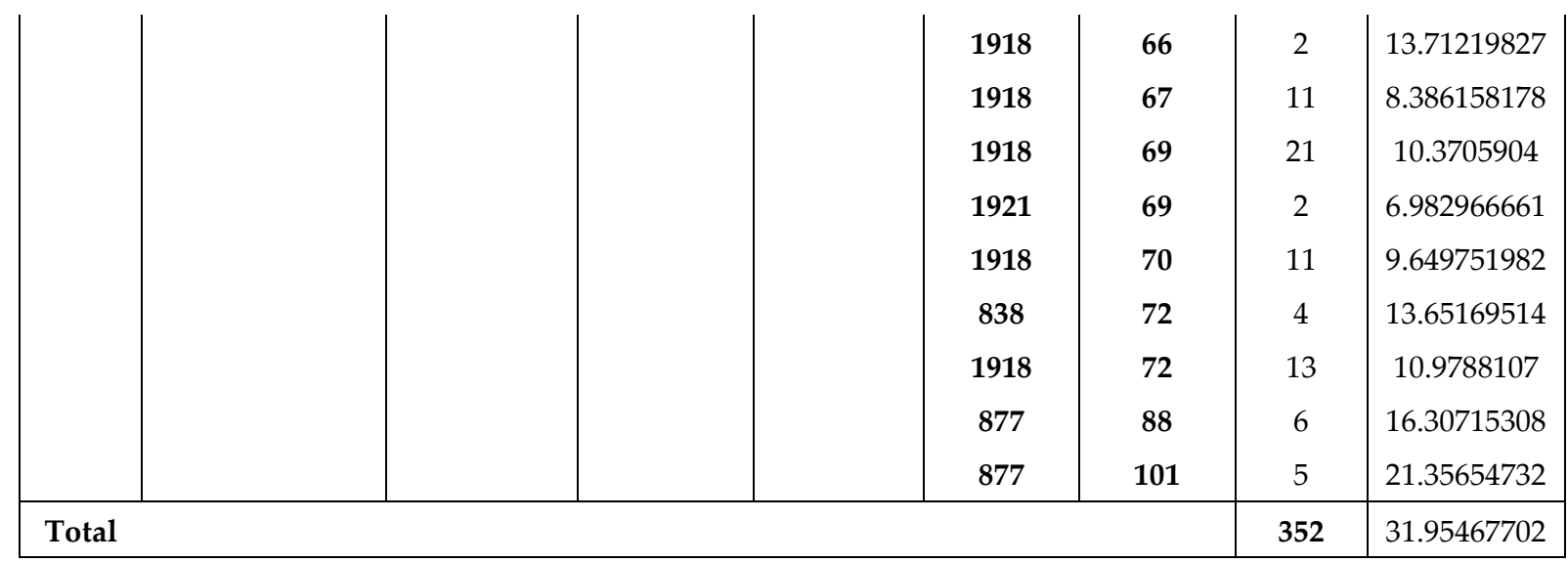


Table 3. Cryo-EM data collection and refinement statistics.

\begin{tabular}{|c|c|c|c|c|c|c|c|}
\hline & \multicolumn{2}{|c|}{$\begin{array}{c}\text { Endogenous FAS } \\
\text { holoenzyme }\end{array}$} & \multicolumn{2}{|l|}{ FAS } & $\begin{array}{c}\text { Reconstituted FAS } \\
\text { holoenzyme }\end{array}$ & \multicolumn{2}{|c|}{$\begin{array}{l}\text { FAS with malonyl- } \\
\text { CoA and NADPH }\end{array}$} \\
\hline Data collection and processing & \multirow{2}{*}{\multicolumn{2}{|c|}{ Titan Krios }} & \multirow{2}{*}{\multicolumn{2}{|c|}{ Titan Krios }} & & \multirow{2}{*}{\multicolumn{2}{|c|}{ Titan Krios }} \\
\hline Microscope & & & & & Titan Krios & & \\
\hline Voltage $(\mathrm{kV})$ & \multicolumn{2}{|l|}{300} & \multicolumn{2}{|c|}{300} & 300 & \multicolumn{2}{|c|}{300} \\
\hline Camera & \multicolumn{2}{|c|}{$\begin{array}{c}\text { Falcon 3EC } \\
\text { (integrated mode) }\end{array}$} & \multicolumn{2}{|c|}{$\begin{array}{c}\text { Falcon 3EC } \\
\text { (integrated mode) }\end{array}$} & $\begin{array}{c}\text { Falcon 3EC } \\
\text { (integrated mode) }\end{array}$ & \multicolumn{2}{|c|}{$\begin{array}{c}\text { Falcon 3EC } \\
\text { (integrated mode) }\end{array}$} \\
\hline Slit width (ev) & \multicolumn{2}{|l|}{ - } & \multicolumn{2}{|l|}{-} & - & \multicolumn{2}{|l|}{ - } \\
\hline Magnification & \multicolumn{2}{|c|}{$132000 \mathrm{x}$} & \multicolumn{2}{|c|}{$132000 x$} & $132000 x$ & \multicolumn{2}{|c|}{$132000 x$} \\
\hline Pixel size $(\AA)$ & \multicolumn{2}{|l|}{0.914} & \multicolumn{2}{|l|}{1.06} & 1.06 & \multicolumn{2}{|l|}{1.06} \\
\hline No. of Frames & \multicolumn{2}{|l|}{39} & \multicolumn{2}{|l|}{20} & 30 & \multicolumn{2}{|l|}{20} \\
\hline Electron exposure (e-/ ̊̊2) & 64 & & 62 & & 48 & 40 & \\
\hline Defocus range $(\mu \mathrm{m})$ & $0.5-3 \mu \mathrm{r}$ & & $1-3 \mu \mathrm{m}$ & & $0.7-3 \mu \mathrm{m}$ & $0.7-3 \mu \mathrm{r}$ & \\
\hline Micrographs acquired & 14968 & & 5441 & & 4593 & 11416 & \\
\hline Initial particle images & 130576 & & 118920 & & 856940 & 216851 & \\
\hline Particle images after 2D classification & 789813 & & 834204 & & 642703 & 167916 & \\
\hline FAS dome conformation & Non-rotated & Rotated & Non-rotated & Rotated & Rotated & Non-rotated & Rotated \\
\hline Final particle images & 81635 & 127305 & 144526 & 69059 & 110597 & 191,899 & 43,467 \\
\hline Symmetry imposed & D3 & D3 & D3 & D3 & D3 & D3 & D3 \\
\hline Resolution $(\AA \AA)$ & 3.3 & 3.2 & 2.9 & 3.2 & 2.8 & 3 & 3.3 \\
\hline FSC threshold & 0.143 & 0.143 & 0.143 & 0.143 & 0.143 & 0.143 & 0.143 \\
\hline Map-sharpening B factor $\left(\AA^{2}\right)$ & -130 & -120 & -120 & -100 & -60 & -120 & -120 \\
\hline Refinement & & & & & & & \\
\hline Initial model used & - & & $\begin{array}{l}\text { FAS crystal } \\
\text { structrue }\end{array}$ & - & FAS crystal structrue & - & - \\
\hline Model composition & & & & & & & \\
\hline Non-hydrogen atoms & - & & 178392 & - & 183564 & - & - \\
\hline Protein residues & - & & 178092 & - & 183216 & - & - \\
\hline Ligands & - & & 300 & - & 348 & - & - \\
\hline Fit to map & & & & & & & \\
\hline Correlation coefficient (entire box) & - & & 0.886 & - & 0.896 & - & - \\
\hline Correlation coefficient (around atoms) & - & & 0.889 & - & 0.9 & - & - \\
\hline Fourier shell correlation (entire box)((̊) & - & & 3.23 & - & 3.17 & - & - \\
\hline Fourier shell correlation (around atoms)( $(\AA)$ & - & & 3.05 & - & 2.97 & - & - \\
\hline Protein Geometry & - & & & - & & - & - \\
\hline RMSD deviation bonds ( $(\AA)$ & - & & 0.0084 & - & 0.0089 & - & - \\
\hline RMSD deviation angles $\left(\left(^{\circ}\right)\right.$ & - & & 1.64 & - & 1.65 & - & - \\
\hline Favored rotamers (\%) & - & & 92.29 & - & 92.78 & - & - \\
\hline Poor rotamers (\%) & - & & 7.71 & - & 7.22 & - & - \\
\hline Ramachandran favored (\%) & - & & 93.38 & - & 93.42 & - & - \\
\hline Ramachandran allowed (\%) & - & & 5.54 & - & 5.58 & - & - \\
\hline Ramachandran outliers (\%) & - & & 1.08 & - & 1 & - & - \\
\hline Clashscore & - & & 3.49 & - & 4.31 & - & - \\
\hline MolProbity score & - & & 2.33 & - & 2.39 & $=$ & - \\
\hline EMRinger score & & & 3.95 & & 4.2 & - & - \\
\hline
\end{tabular}


Table 4. Crystallographic data collection and refinement statistics.

\begin{tabular}{|c|c|c|c|}
\hline Tag & FAS & $\begin{array}{c}\text { FAS (anisotropic } \\
\text { cutoff) }\end{array}$ & $\begin{array}{c}\text { FAS } \\
\text { holoenzyme }\end{array}$ \\
\hline \multicolumn{4}{|l|}{ Data Collection } \\
\hline Wavelength & & 0.976 & \\
\hline Resolution range & $191.5-2.9(3.0-2.9)$ & $191.5-2.82(2.9-2.82)$ & $192.5-4.6(4.7-4.6)$ \\
\hline Space group & & $\mathrm{P} 2_{1}$ & \\
\hline \multicolumn{4}{|l|}{ Unit cell } \\
\hline $\mathrm{a}(\AA)$ & 217.6 & 217.6 & 234.9 \\
\hline$b(\AA)$ & 347.5 & 347.5 & 430.3 \\
\hline$c(\AA)$ & 265.2 & 265.2 & 422.6 \\
\hline$\beta\left(^{\circ}\right)$ & 107.88 & 107.8 & 97.01 \\
\hline Total reflections & $5950624(592351)$ & $6471160(645835)$ & $3314706(336434)$ \\
\hline Unique reflections & $822268(81944)$ & $894158(4914)$ & $458021(45686)$ \\
\hline Multiplicity & $7.2(7.2)$ & $7.2(7.2)$ & $7.2(7.4)$ \\
\hline Completeness (\%) (Spherical truncation) & $99.64(99.58)$ & $78.71(5.49)$ & $99.51(99.71)$ \\
\hline Completeness (\%) (ellipsoidal truncation) & - & $96.5(76.5)$ & - \\
\hline Mean I/sigma(I) & $11.81(0.87)$ & $10.91(0.65)$ & $7.95(0.87)$ \\
\hline$R_{\text {pim }}$ & $0.06705(0.8368)$ & $0.07272(1.096)$ & $0.08786(0.8292)$ \\
\hline $\mathrm{CC}_{1 / 2}$ & $0.997(0.341)$ & $0.996(0.241)$ & $0.998(0.364)$ \\
\hline \multicolumn{4}{|l|}{ Refinement in Refmac5 } \\
\hline Number of TLS groups & 59 & 59 & - \\
\hline R-work & $0.1969(0.377)$ & $0.1924(0.3755)$ & $0.2516(0.4139)$ \\
\hline R-free & $0.2183(0.382)$ & $0.2113(0.3583)$ & $0.3098(0.4161)$ \\
\hline Number of non-hydrogen atoms & 179483 & 179483 & 231312 \\
\hline Protein & 178242 & 178242 & 231312 \\
\hline Ligands & 913 & 913 & - \\
\hline Solvent & 328 & 328 & - \\
\hline Protein residues & 22778 & 22778 & 46920 \\
\hline Average B-factor (TLS/Isotropic refinement) $\left(\AA^{2}\right)$ & $49.69 / 92.72$ & $31.66 / 73.36$ & 256.34 \\
\hline Protein & $49.53 / 92.72$ & $31.47 / 73.38$ & 256.34 \\
\hline Ligands & $78.74 / 100.82$ & $66.33 / 80.57$ & - \\
\hline Solvent & $56.07 / 56.07$ & $38.93 / 38.93$ & - \\
\hline Wilson & 78.87 & 59.85 & 218.11 \\
\hline R.m.s.d. bond length $(\AA)$ & 0.014 & 0.014 & 0.016 \\
\hline R.m.s.d. bond angles $(\AA)$ & 1.95 & 1.9 & 1.87 \\
\hline Ramachandran favored (\%) & 94.1 & 94.51 & 93.86 \\
\hline Ramachandran allowed (\%) & 5.26 & 4.88 & 5.79 \\
\hline Ramachandran outliers (\%) & 0.64 & 0.61 & 0.35 \\
\hline Rotamer outliers (\%) & 13.8 & 13.7 & - \\
\hline Clashscore & 7.49 & 7.36 & 0.41 \\
\hline
\end{tabular}

Values in parenthesis denote values for the highest resolution shell.

Number of unique reflections used in refinement is shown. No rejections were applied. 
Table 5. Cryo-EM data collection for conformational energy landscape analysis.

\begin{tabular}{|c|c|c|c|c|c|c|c|c|}
\hline \multirow[b]{2}{*}{ Data collection and processing } & \multicolumn{8}{|c|}{ Conformational energy landscape analysis } \\
\hline & no $\gamma$-subunit & $2 x \gamma$-subunit & $4 x \gamma$-subunit & $6 \times \gamma$-subunit & $12 \times \gamma$-subunit & $24 \times \gamma$-subunit & $48 \times \gamma$-subunit & $96 \times \gamma$-subunit \\
\hline Microscope & Titan Krios & Titan Krios & Titan Krios & Titan Krios & Titan Krios & Titan Krios & Titan Krios & Titan Krios \\
\hline Voltage (kV) & 300 & 300 & 300 & 300 & 300 & 300 & 300 & 300 \\
\hline Camera & $\begin{array}{l}\text { K2 with GIF } \\
\text { (counting }\end{array}$ & $\begin{array}{c}\text { Falcon 3EC } \\
\text { (integrated mode) }\end{array}$ & $\mid \begin{array}{c}\text { Falcon 3EC } \\
\text { (integrated mode) }\end{array}$ & $\begin{array}{l}\text { K2 with GIF } \\
\text { (counting }\end{array}$ & $\begin{array}{l}\text { K2 with GIF } \\
\text { (counting }\end{array}$ & $\begin{array}{l}\text { K2 with GIF } \\
\text { (counting }\end{array}$ & $\begin{array}{l}\text { K2 with GIF } \\
\text { (counting }\end{array}$ & $\begin{array}{l}\text { K2 with GIF } \\
\text { (counting }\end{array}$ \\
\hline Slit width (ev) & 20 & - & - & 20 & 20 & 20 & 20 & 20 \\
\hline Magnification & $165,000 \mathrm{x}$ & $132000 \mathrm{X}$ & $132000 \mathrm{X}$ & $130,000 \mathrm{X}$ & $130,000 \mathrm{x}$ & $130,000 x$ & $130,000 x$ & $130,000 \mathrm{X}$ \\
\hline Pixel size $(\AA)$ & 0.815 & 1.06 & 1.06 & 1.05 & 1.05 & 1.05 & 1.05 & 1.05 \\
\hline No. of Frames & 40 & 20 & 20 & 40 & 40 & 40 & 40 & 40 \\
\hline Electron exposure $\left(\mathrm{e}-/ \AA^{2}\right)$ & 50 & 30 & 30 & 46 & 46 & 46 & 46 & 46 \\
\hline Defocus range $(\mu \mathrm{m})$ & $0.5-2.5 \mu \mathrm{m}$ & $0.7-3 \mu \mathrm{m}$ & $0.8-2.5 \mu \mathrm{m}$ & $0.6-2.2 \mu \mathrm{m}$ & $0.5-2.5 \mu \mathrm{m}$ & $0.5-2.6 \mu \mathrm{m}$ & $0.7-2.5 \mu \mathrm{m}$ & $0.5-2.6 \mu \mathrm{m}$ \\
\hline Micrographs acquired & 2947 & 2167 & 1802 & 1329 & 1570 & 1961 & 1652 & 2263 \\
\hline Initial particle images & 222903 & 419527 & 350330 & 164767 & 172994 & 240306 & 220995 & 310814 \\
\hline $\begin{array}{l}\text { Particle images after 2D } \\
\text { classification }\end{array}$ & 114553 & 247996 & 230491 & 90050 & 100589 & 69904 & 27918 & 27649 \\
\hline $\begin{array}{l}\text { No. of Classes for } \\
\text { conformational landscapes }\end{array}$ & 25 & 30 & 30 & 20 & 20 & 20 & 10 & 10 \\
\hline Symmetry imposed & D3 & D3 & D3 & D3 & D3 & D3 & D3 & D3 \\
\hline
\end{tabular}




\section{B. Supplementary figures}

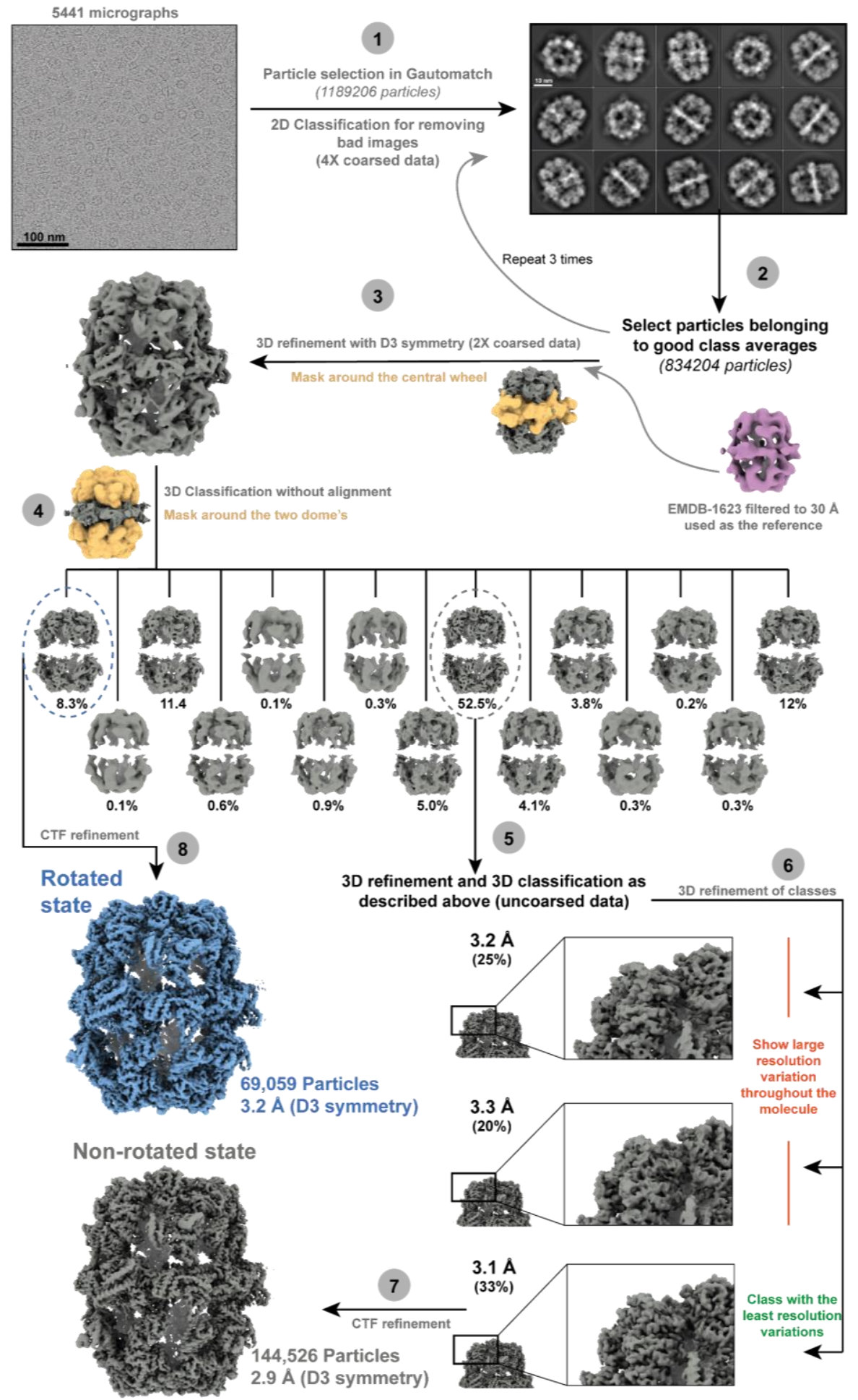

Figure S1. Scheme for cryo-EM data processing for FAS. The scheme depicts the different image processing steps performed. (1) Motion-corrected and dose weighted micrographs were used for particle selection. (2) Selected, individual particles were classified in 2D for 3 rounds where particles belonging to bad classes (resulting from bad optical properties or low signal-to-noise) were removed 
after each step. (3) The selected particles were then used for 3D refinement using D3 symmetry with the published cryo-EM structure of Saccharomyces cerevisiae FAS (EMDB - 1623) as a reference. During the 3D refinement, a mask was placed around the central wheel to focus the refinement around the most stable part of the protein. (4) The alignment parameter of the particles from the previous step were used for classifying the particles into 15 classes. For classification, only the dome of the FAS was considered to sort out the major movements of this molecule. (5) Particles from the best $3 D$ class with a non-rotated dome conformation were selected followed by another round of $3 D$ refinement and classification as described above. (6) Particles from classes with resolutions below 4 $\AA$ after $3 D$ classification were refined again. (7-8) CTF refinement of the respective particles belonging to the class with the most uniform resolution was performed followed by a final $3 D$ refinement. The final 3D maps obtained had an overall resolution of $2.9 \AA$ for the non-rotated FAS conformation (grey) and $3.2 \AA$ for the rotated FAS conformation (blue).

A

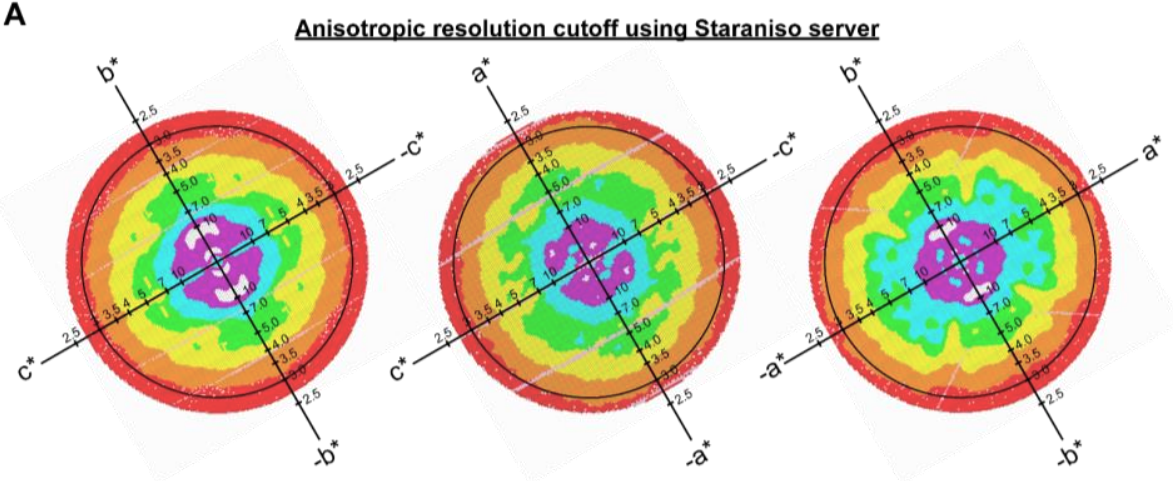

B
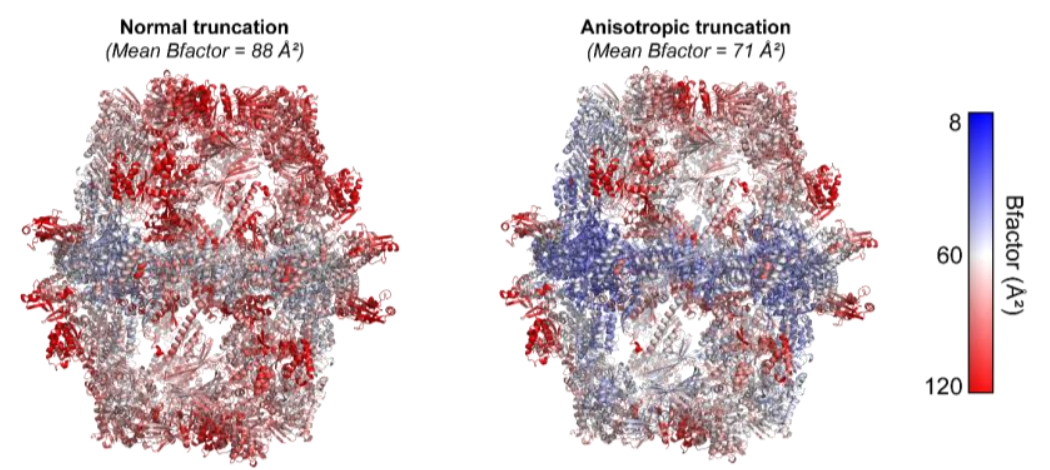

Figure S2. Analysis of the FAS X-ray dataset for anisotropic diffraction using the STARANISO server. (i) Diffraction intensity plots along the three crystallographic axes. Note the strongest diffraction is along the $a^{*}$-axis $(2.8 \AA)$, followed by the $c^{*}$-axis $(3.0 \AA)$ and the weakest diffraction is along the $b^{*}$-axis $(3.1 \AA)$. Since the crystal is nearly isometric in all three dimensions, this mild anisotropy is likely to represent the difference in unit cell numbers along the three crystallographic axes. (ii) B-factor distribution of main chain atoms of the refined $F A S$ model against data spherically truncated at $2.9 \AA$ (left) and anisotropically truncated as indicated in (i) (right). A distinct drop in mean B-factors is visible upon anisotropic truncation of the data. 


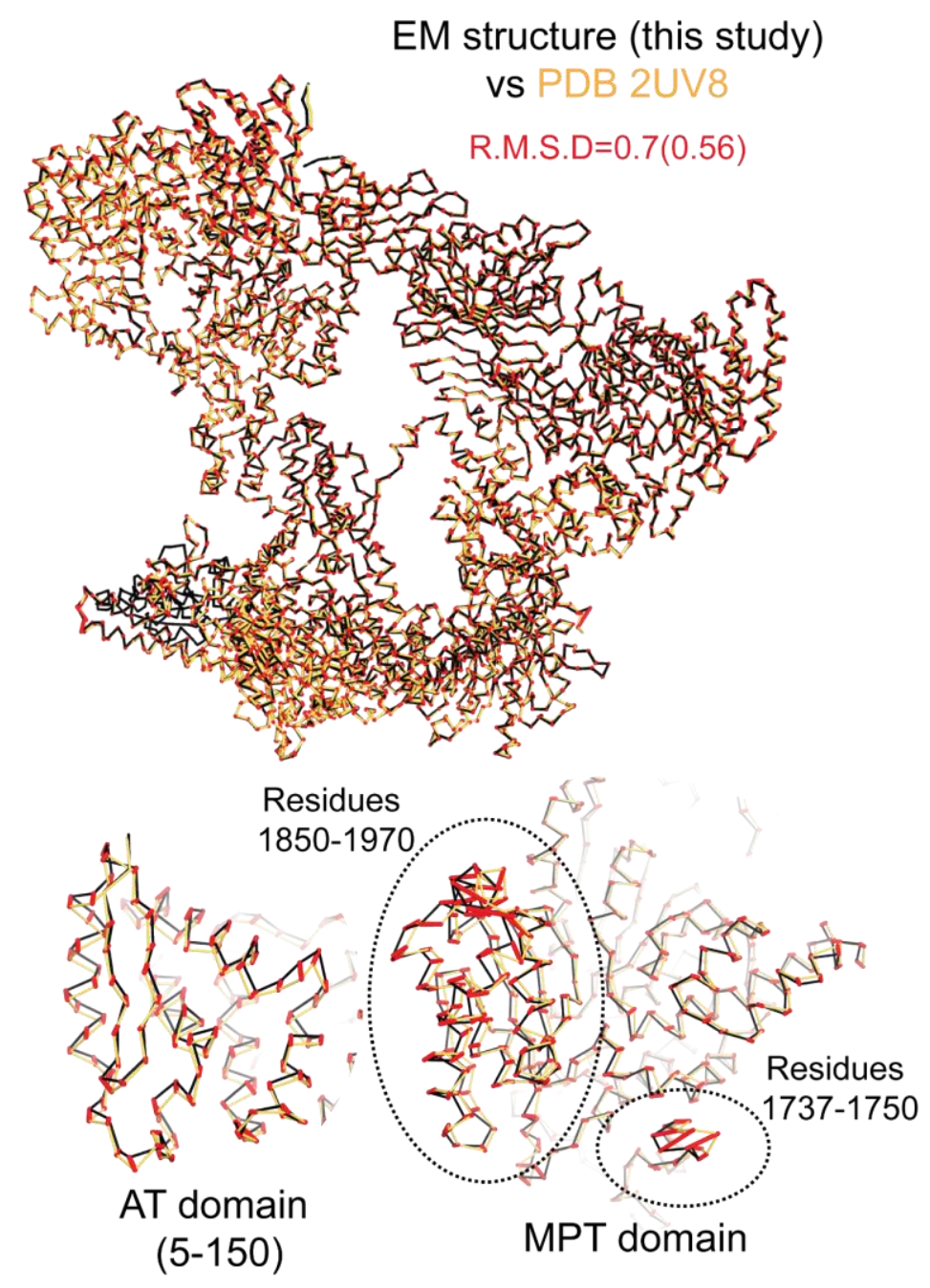

Figure S3. Comparison between the cryo-EM FAS model and the published X-ray crystallographic FAS structure. The RMSD between the cryo-EM from this study and the published FAS structure (PDB 2UV8) was less than or equal to $7 \AA$. The AT domains residues 5150 along with MPT domain residues 1737-1750 and 1850-1970 contributed towards 20-30\% of the total deviations among the structures. RMSD values calculated excluding the aforementioned residues are indicated within brackets. 

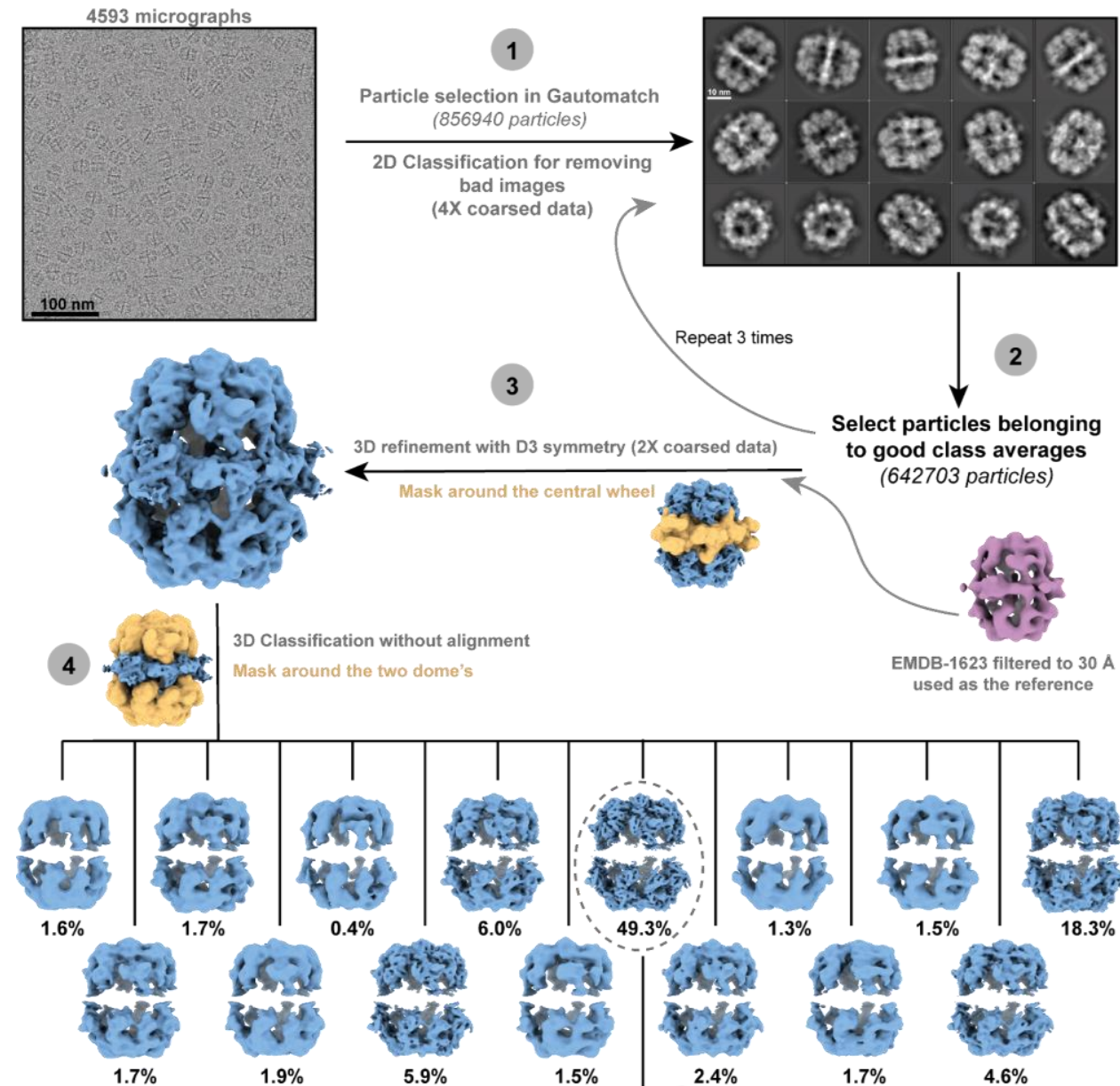

EMDB-1623 filtered to $30 \mathrm{~A}$ used as the reference
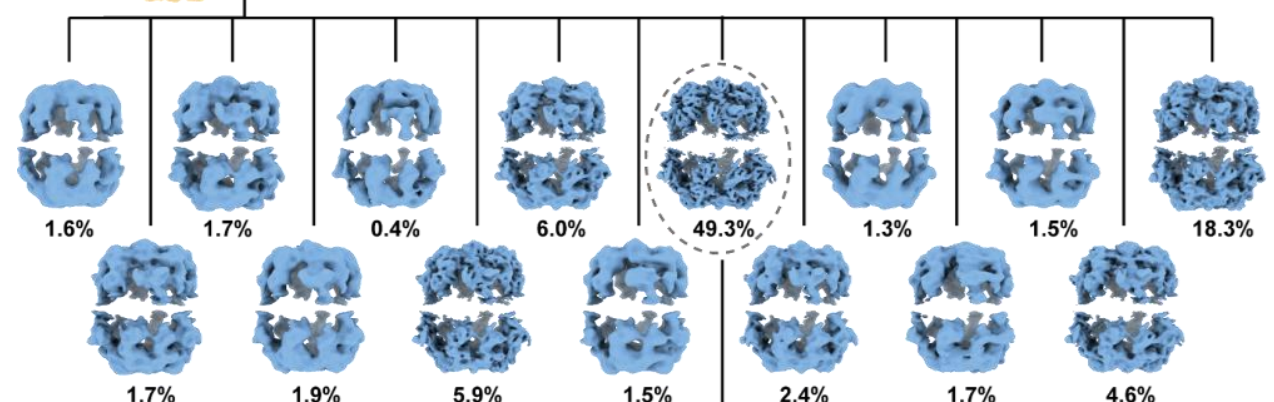

6

3D refinement and 3D classification as

3D refinement of classes
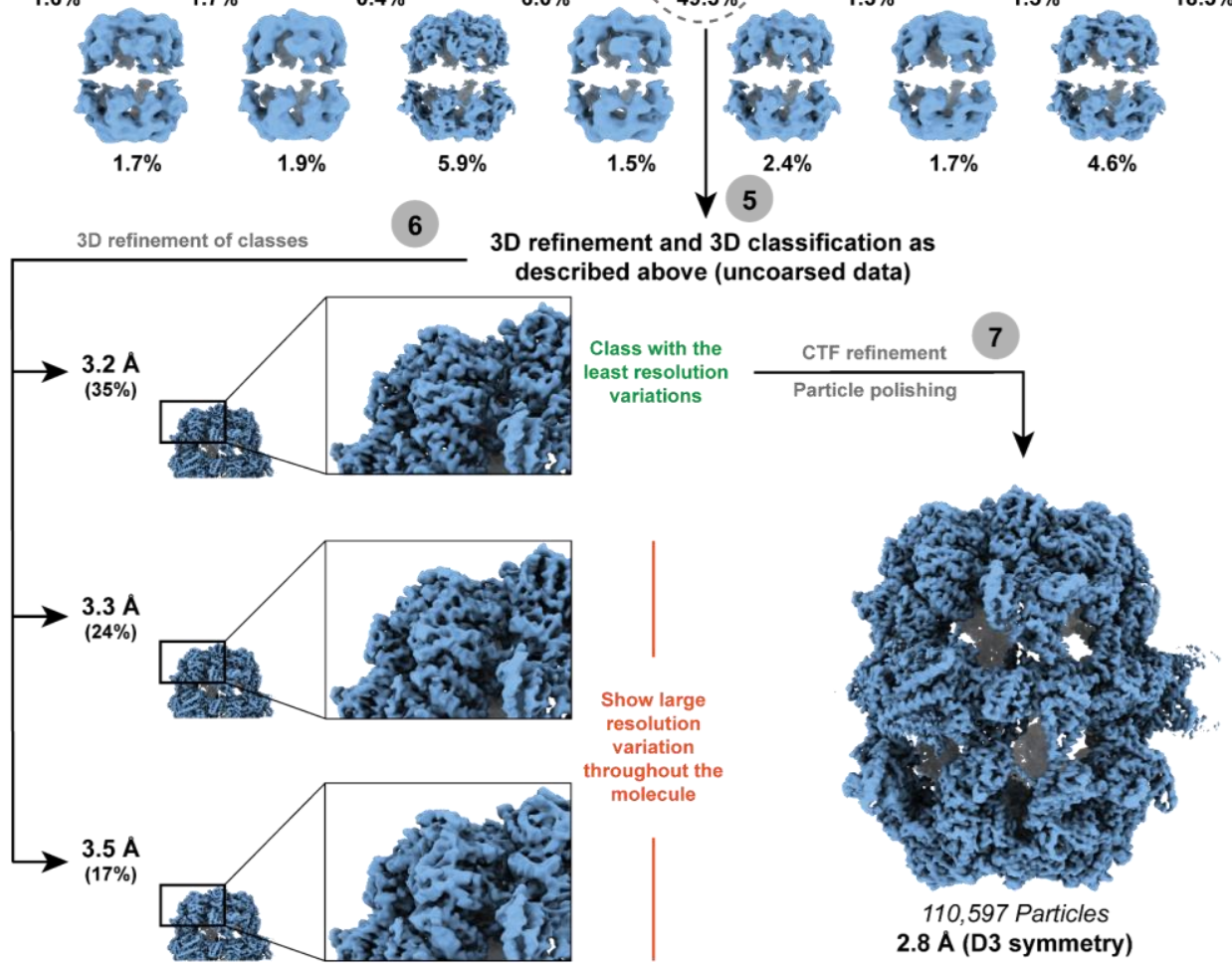

Figure S4. Scheme for cryo-EM data processing for FAS Holoenzyme complex. The scheme depicts the different image processing steps performed. The processing pipeline was identical to the one used for the FAS complex (Supplementary figure S1). For this dataset, after CTF refinement, particle polishing was performed in Relion for the particles used in the final $3 \mathrm{D}$ refinement step. The final 3D map obtained for this complex had an overall resolution of $2.8 \AA$ as determined by the FSC 0.143 criterion. 


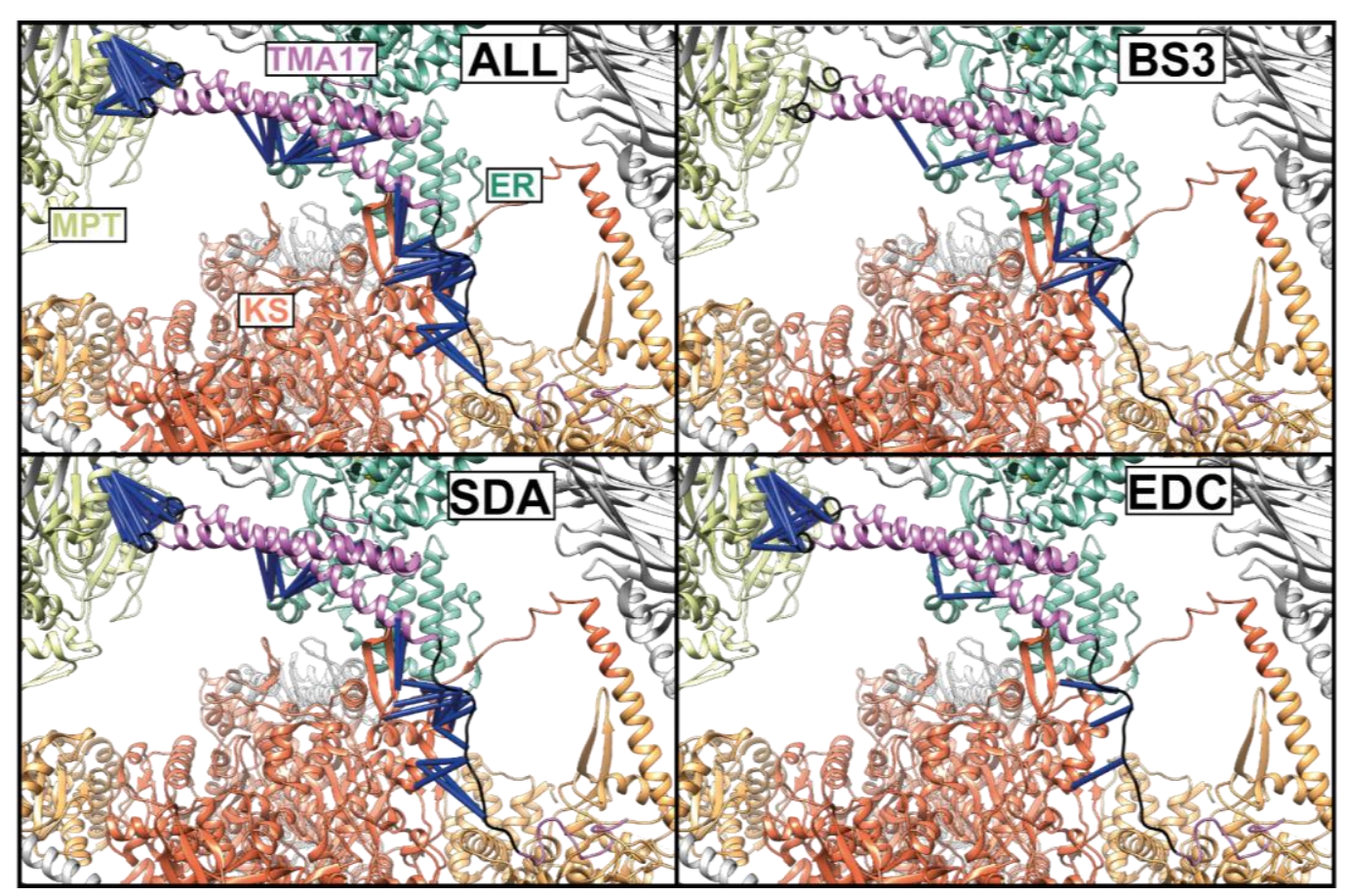

Figure S5. XL-MS validation of the $\gamma$-subunit model. Crosslinks mapped on the final FAS holoenzyme structure for BS3 (top right), SDA (bottom left) and EDC (bottom right) are shown. The crosslinks are indicated by the blue rods. Only the crosslinks between residues within the distance threshold of $20 \AA$ for the modelled part of the $\gamma$-subunit and $30 \AA$ for the un-modelled part of the $\gamma$-subunit are depicted. 
(i)
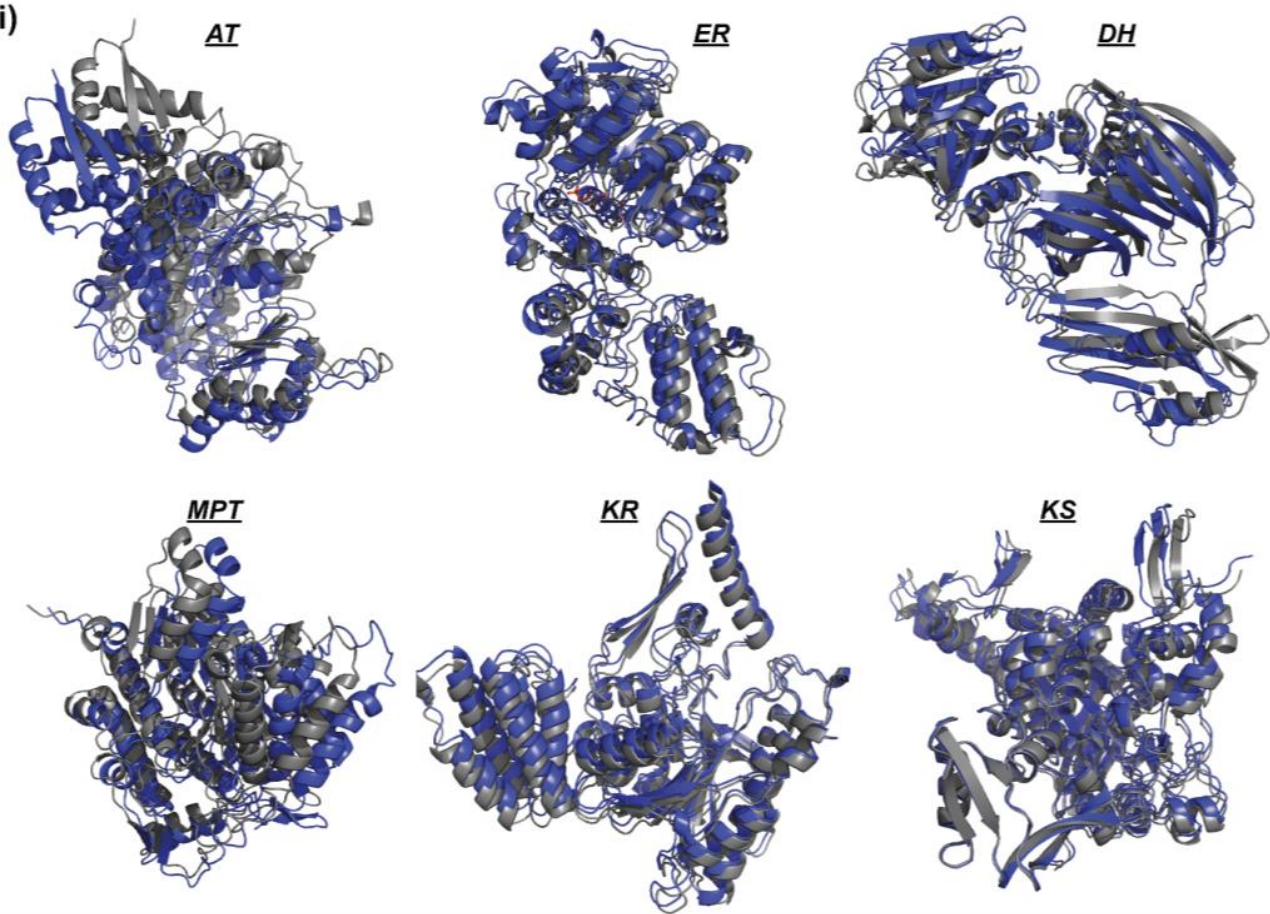

(ii)
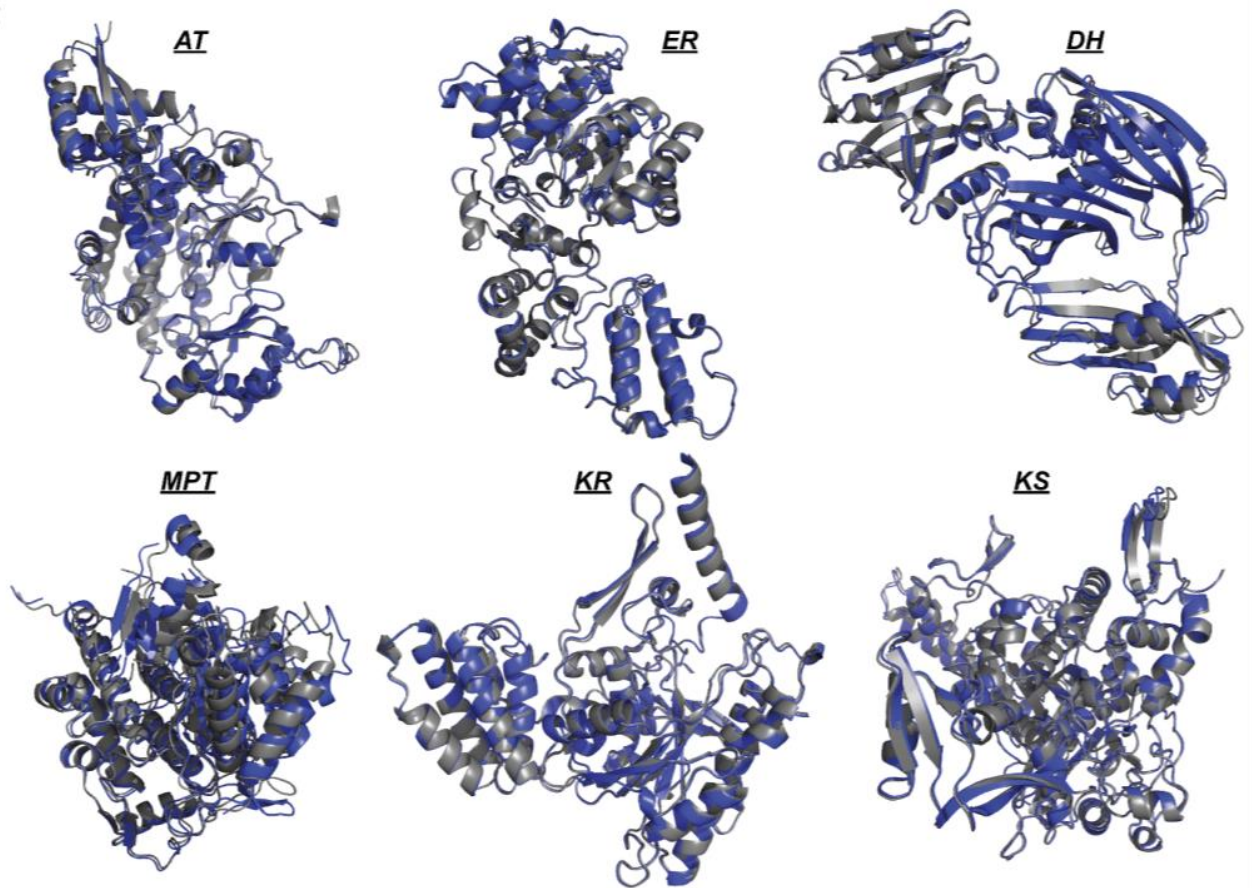

Figure S6. Overlay of FAS models from the non-rotated and rotated conformation. (i) Shown are overlays of domains of the non-rotated (grey) vs. the rotated conformation of the FAS (blue) when the two structures are aligned with respect to the central wheel. (ii) When the isolated domains are aligned to each other, it is notable that all domains superpose well, aside from minute rigid body shifts seen in the MPT domain. This allows the conclusion that the conformational change in the FAS is elicited by rigid-body motions, rather than re-structuring of enzymatic domains. 
(i)
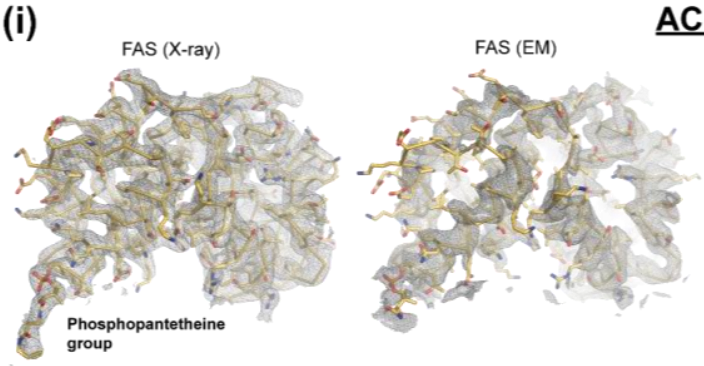

$\underline{A C P}$

(ii)
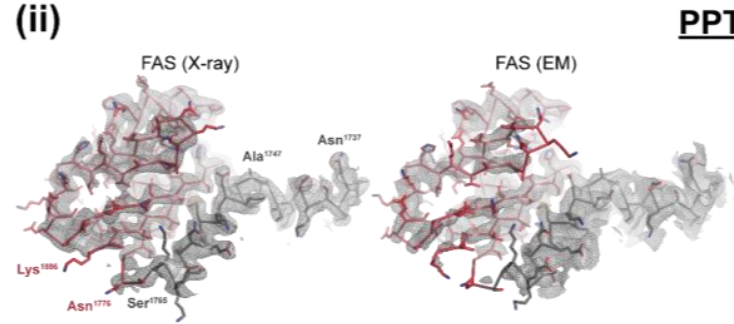

$\underline{\text { PPT }}$
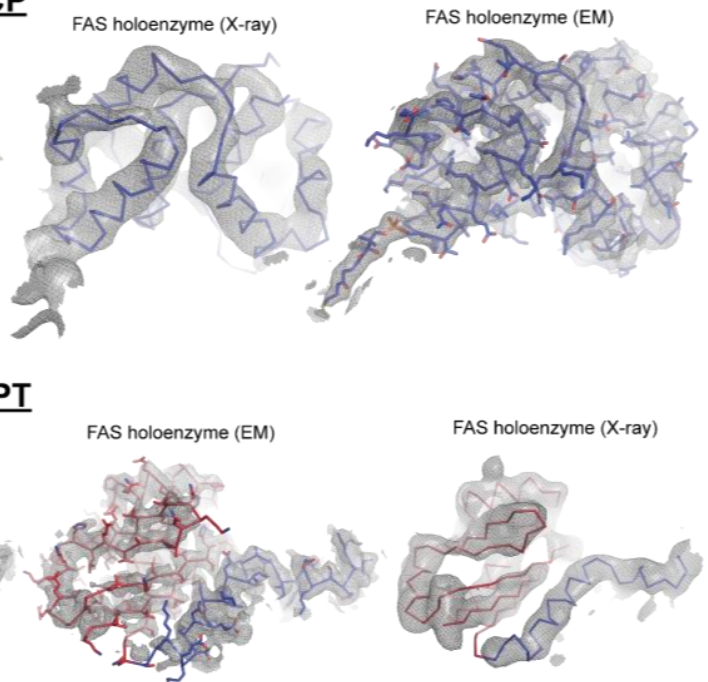

Figure S7. Comparison of ACP- and PPT-domain models in the FAS and FAS holoenzyme structures, as determined by X-ray crystallography and cryo-EM. (i) ACP domain models with their corresponding density from the crystallographic and cryo-EM structures of FAS (yellow) and the FAS holoenzyme (blue). In contrast to the previously published structures of the yeast FAS (Anselmi et al., 2010; Johansson et al., 2008; Leibundgut et al., 2007), the full phosphopantetheine prosthetic group attached to Ser180 was visible in the FAS crystallographic structure and the FAS holoenzyme cryo-EM structure reported herein. (ii) PPT domain (red) models with their corresponding density from the crystallographic and cryo-EM structures of FAS (left) and FAS holoenzyme (right). The C-terminal segment of the $\alpha$-subunit comprising of an $\alpha$-helical segment (Ala1747-Ser1765) and the PPT domain (Asn1776-Lys1886) present here, were elusive in the previous reported high resolution structures of the fungal FAS (Jenni et al., 2007; Leibundgut et al., 2007). 


\section{References}

Adams, P.D., Afonine, P. V., Bunkóczi, G., Chen, V.B., Davis, I.W., Echols, N., Headd, J.J., Hung, L.-W., Kapral, G.J., Grosse-Kunstleve, R.W., et al. (2010). PHENIX : a comprehensive Python-based system for macromolecular structure solution. Acta Crystallogr. Sect. D Biol. Crystallogr. 66, 213-221.

Albuquerque, C.P., Smolka, M.B., Payne, S.H., Bafna, V., Eng, J., and Zhou, H. (2008). A Multidimensional Chromatography Technology for In-depth Phosphoproteome Analysis. Mol. Cell. Proteomics 7, 1389-1396.

Anselmi, C., Grininger, M., Gipson, P., and Faraldo-Goěmez, J.D. (2010). Mechanism of substrate shuttling by the Acyl-carrier protein within the fatty acid mega-synthase. J. Am. Chem. Soc. 132, 12357-12364.

Beld, J., Lee, D.J., and Burkart, M.D. (2015). Fatty acid biosynthesis revisited: structure elucidation and metabolic engineering. Mol. Biosyst. 11, 38-59.

Bertram, K., Agafonov, D.E., Liu, W.-T., Dybkov, O., Will, C.L., Hartmuth, K., Urlaub, H., Kastner, B., Stark, H., and Lührmann, R. (2017). Cryo-EM structure of a human spliceosome activated for step 2 of splicing. Nature 542, 318-323.

Blees, A., Januliene, D., Hofmann, T., Koller, N., Schmidt, C., Trowitzsch, S., Moeller, A., and Tampé, R. (2017). Structure of the human MHC-I peptide-loading complex. Nature 551, $525-528$.

Brown, A.P., Slabas, A.R., and Rafferty, J.B. (2009). Fatty Acid Biosynthesis in Plants Metabolic Pathways, Structure and Organization. (Springer, Dordrecht), pp. 11-34.

Cesa, L.C., Mapp, A.K., and Gestwicki, J.E. (2015). Direct and Propagated Effects of Small Molecules on Protein-Protein Interaction Networks. Front. Bioeng. Biotechnol. 3, 119.

Chan, D.I., and Vogel, H.J. (2010). Current understanding of fatty acid biosynthesis and the acyl carrier protein. Biochem. J. 430, 1-19.

Chari, A., Haselbach, D., Kirves, J.-M., Ohmer, J., Paknia, E., Fischer, N., Ganichkin, O., 
Möller, V., Frye, J.J., Petzold, G., et al. (2015). ProteoPlex: stability optimization of macromolecular complexes by sparse-matrix screening of chemical space. Nat. Methods 12, 859-865.

Cheng, Y. (2015). Single-Particle Cryo-EM at Crystallographic Resolution. Cell 161, 450-457.

Chirala, S.S., Huang, W.Y., Jayakumar, A., Sakai, K., Wakil, S.J., Mahon, K., Finegold, M., and Wakil, S.J. (1997). Animal fatty acid synthase: functional mapping and cloning and expression of the domain I constituent activities. Proc. Natl. Acad. Sci. U. S. A. 94, 55885593.

Davis, M.S., Cronan, J.E., and Jr. (2001). Inhibition of Escherichia coli acetyl coenzyme A carboxylase by acyl-acyl carrier protein. J. Bacteriol. 183, 1499-1503.

Dmitriev, O.Y., Lutsenko, S., and Muyldermans, S. (2016). Nanobodies as Probes for Protein Dynamics in Vitro and in Cells. J. Biol. Chem. 291, 3767-3775.

Duhoo, Y., Roche, J., Trinh, T.T.N., Desmyter, A., Gaubert, A., Kellenberger, C., Cambillau, C., Roussel, A., Leone, P., and IUCr (2017). Camelid nanobodies used as crystallization chaperones for different constructs of PorM, a component of the type IX secretion system from Porphyromonas gingivalis. Acta Crystallogr. Sect. F Struct. Biol. Commun. 73, 286-293.

Egner, R., Thumm, M., Straub, M., Simeon, A., Schuller, H.J., and Wolf, D.H. (1993). Tracing intracellular proteolytic pathways. Proteolysis of fatty acid synthase and other cytoplasmic proteins in the yeast Saccharomyces cerevisiae. J. Biol. Chem. 268, 27269-27276.

Elad, N., Baron, S., Peleg, Y., Albeck, S., Grunwald, J., Raviv, G., Shakked, Z., Zimhony, O., and Diskin, R. (2018). Structure of Type-I Mycobacterium tuberculosis fatty acid synthase at $3.3 \AA$ resolution. Nat. Commun. 9, 3886.

Emsley, P., and Cowtan, K. (2004). Coot : model-building tools for molecular graphics. Acta Crystallogr. Sect. D Biol. Crystallogr. 60, 2126-2132.

Fischer, N., Konevega, A.L., Wintermeyer, W., Rodnina, M. V., and Stark, H. (2010). Ribosome dynamics and tRNA movement by time-resolved electron cryomicroscopy. Nature 466, 329-333. 
Fleischer, T.C., Weaver, C.M., McAfee, K.J., Jennings, J.L., and Link, A.J. (2006). Systematic identification and functional screens of uncharacterized proteins associated with eukaryotic ribosomal complexes. Genes Dev. 20, 1294-1307.

Förster, A., Masters, E.I., Whitby, F.G., Robinson, H., and Hill, C.P. (2005). The $1.9 \AA$ Structure of a Proteasome-11S Activator Complex and Implications for ProteasomePAN/PA700 Interactions. Mol. Cell 18, 589-599.

Gajewski, J., Buelens, F., Serdjukow, S., Janßen, M., Cortina, N., Grubmüller, H., and Grininger, M. (2017a). Engineering fatty acid synthases for directed polyketide production. Nat. Chem. Biol. 13, 363-365.

Gajewski, J., Pavlovic, R., Fischer, M., Boles, E., and Grininger, M. (2017b). Engineering fungal de novo fatty acid synthesis for short chain fatty acid production. Nat. Commun. 8 , 14650.

Gietz, R.D. (2014). Yeast transformation by the LiAc/SS carrier DNA/PEG method. Methods Mol. Biol. 1163, 33-44.

Gipson, P., Mills, D.J., Wouts, R., Grininger, M., Vonck, J., and Kuhlbrandt, W. (2010). Direct structural insight into the substrate-shuttling mechanism of yeast fatty acid synthase by electron cryomicroscopy. Proc. Natl. Acad. Sci. U. S. A. 107, 9164-9169.

Goddard, T.D., Huang, C.C., Meng, E.C., Pettersen, E.F., Couch, G.S., Morris, J.H., and Ferrin, T.E. (2018). UCSF ChimeraX: Meeting modern challenges in visualization and analysis. Protein Sci. 27, 14-25.

Grigorieff, N. (2016). Frealign: An Exploratory Tool for Single-Particle Cryo-EM. Methods Enzymol. 579, 191-226.

Groll, M., Ditzel, L., Löwe, J., Stock, D., Bochtler, M., Bartunik, H.D., and Huber, R. (1997). Structure of $20 S$ proteasome from yeast at $2.4 \AA$ resolution. Nature $386,463-471$.

Hanssum, A., Zhong, Z., Rousseau, A., Krzyzosiak, A., Sigurdardottir, A., and Bertolotti, A. (2014). An inducible chaperone adapts proteasome assembly to stress. Mol. Cell 55, 566577. 
Harshbarger, W., Miller, C., Diedrich, C., and Sacchettini, J. (2015). Crystal Structure of the Human 20S Proteasome in Complex with Carfilzomib. Structure 23, 418-424.

Haselbach, D., Schrader, J., Lambrecht, F., Henneberg, F., Chari, A., and Stark, H. (2017). Long-range allosteric regulation of the human $26 S$ proteasome by $20 \mathrm{~S}$ proteasome-targeting cancer drugs. Nat. Commun. 8, 15578.

Haselbach, D., Komarov, I., Agafonov, D.E., Hartmuth, K., Graf, B., Dybkov, O., Urlaub, H., Kastner, B., Lührmann, R., and Stark, H. (2018). Structure and Conformational Dynamics of the Human Spliceosomal Bact Complex. Cell 172, 454-464.e11.

Hasslacher, M., Ivessa, A.S., Paltauf, F., and Kohlwein, S.D. (1993). Acetyl-CoA carboxylase from yeast is an essential enzyme and is regulated by factors that control phospholipid metabolism. J. Biol. Chem. 268, 10946-10952.

Hedbacker, K., and Carlson, M. (2008). SNF1/AMPK pathways in yeast. Front. Biosci. 13, $2408-2420$.

Herbst, D.A., Townsend, C.A., and Maier, T. (2018). The architectures of iterative type I PKS and FAS. Nat. Prod. Rep. 35, 1046-1069.

Holt, L.J., Tuch, B.B., Villén, J., Johnson, A.D., Gygi, S.P., and Morgan, D.O. (2009). Global analysis of Cdk1 substrate phosphorylation sites provides insights into evolution. Science $325,1682-1686$.

Hunkeler, M., Stuttfeld, E., Hagmann, A., Imseng, S., and Maier, T. (2016). The dynamic organization of fungal acetyl-CoA carboxylase. Nat. Commun. 7, 11196.

Janßen, H.J., and Steinbüchel, A. (2014). Fatty acid synthesis in Escherichia coli and its applications towards the production of fatty acid based biofuels. Biotechnol. Biofuels 7, 7.

Jenni, S., Leibundgut, M., Boehringer, D., Frick, C., Mikolásek, B., and Ban, N. (2007). Structure of fungal fatty acid synthase and implications for iterative substrate shuttling. Science 316, 254-261.

Johansson, P., Wiltschi, B., Kumari, P., Kessler, B., Vonrhein, C., Vonck, J., Oesterhelt, D., and Grininger, M. (2008). Inhibition of the fungal fatty acid synthase type I multienzyme 
complex. Proc. Natl. Acad. Sci. U. S. A. 105, 12803-12808.

Johansson, P., Mulinacci, B., Koestler, C., Vollrath, R., Oesterhelt, D., and Grininger, M. (2009). Multimeric Options for the Auto-Activation of the Saccharomyces cerevisiae FAS Type I Megasynthase. Structure 17, 1063-1074.

Kabsch, W. (2010). XDS. Acta Crystallogr. Sect. D 66, 125-132.

Kastner, B., Fischer, N., Golas, M.M., Sander, B., Dube, P., Boehringer, D., Hartmuth, K., Deckert, J., Hauer, F., Wolf, E., et al. (2008). GraFix: sample preparation for single-particle electron cryomicroscopy. Nat. Methods 5, 53-55.

Kastritis, P.L., O’Reilly, F.J., Bock, T., Li, Y., Rogon, M.Z., Buczak, K., Romanov, N., Betts, M.J., Bui, K.H., Hagen, W.J., et al. (2017). Capturing protein communities by structural proteomics in a thermophilic eukaryote. Mol. Syst. Biol. 13, 936.

Kim, D.E., Chivian, D., and Baker, D. (2004). Protein structure prediction and analysis using the Robetta server. Nucleic Acids Res. 32, W526-31.

Kufareva, I., and Abagyan, R. (2012). Methods of protein structure comparison. Methods Mol. Biol. 857, 231-257.

Lebedev, A.A., Young, P., Isupov, M.N., Moroz, O. V, Vagin, A.A., and Murshudov, G.N. (2012). JLigand: a graphical tool for the CCP4 template-restraint library. Acta Crystallogr. D. Biol. Crystallogr. 68, 431-440.

Leibundgut, M., Jenni, S., Frick, C., and Ban, N. (2007). Structural basis for substrate delivery by acyl carrier protein in the yeast fatty acid synthase. Science 316, 288-290.

Liu, W., Song, H., Chen, Q., Yu, J., Xian, M., Nian, R., and Feng, D. (2018). Recent advances in the selection and identification of antigen-specific nanobodies. Mol. Immunol. 96, 37-47. Liu, Y., Ranish, J.A., Aebersold, R., and Hahn, S. (2001). Yeast Nuclear Extract Contains Two Major Forms of RNA Polymerase II Mediator Complexes. J. Biol. Chem. 276, 7169-7175.

Lomakin, I.B., Xiong, Y., and Steitz, T.A. (2007). The crystal structure of yeast fatty acid synthase, a cellular machine with eight active sites working together. Cell 129, 319-332. 
Lynen, F. (1980). On the Structure of Fatty Acid Synthetase of Yeast. Eur. J. Biochem. 112, $431-442$.

Maier, T., Leibundgut, M., and Ban, N. (2008). The crystal structure of a mammalian fatty acid synthase. Science 321, 1315-1322.

Murphy, J.P., Stepanova, E., Everley, R.A., Paulo, J.A., and Gygi, S.P. (2015). Comprehensive Temporal Protein Dynamics during the Diauxic Shift in Saccharomyces cerevisiae. Mol. Cell. Proteomics 14, 2454-2465.

Murshudov, G.N., Skubák, P., Lebedev, A.A., Pannu, N.S., Steiner, R.A., Nicholls, R.A., Winn, M.D., Long, F., and Vagin, A.A. (2011). REFMAC5 for the refinement of macromolecular crystal structures. Acta Crystallogr. D. Biol. Crystallogr. 67, 355-367.

Muyldermans, S. (2013). Nanobodies: Natural Single-Domain Antibodies. Annu. Rev. Biochem. 82, 775-797.

Notredame, C., Higgins, D.G., and Heringa, J. (2000). T-coffee: A novel method for fast and accurate multiple sequence alignment. J. Mol. Biol. 302, 205-217.

Di Pasquale, M.G. (2009). The Essentials of Essential Fatty Acids. J. Diet. Suppl. 6, 143-161.

Peralta-Yahya, P.P., Zhang, F., del Cardayre, S.B., and Keasling, J.D. (2012). Microbial engineering for the production of advanced biofuels. Nature 488, 320-328.

Pettersen, E.F., Goddard, T.D., Huang, C.C., Couch, G.S., Greenblatt, D.M., Meng, E.C., and Ferrin, T.E. (2004). UCSF Chimera: A visualization system for exploratory research and analysis. J. Comput. Chem. 25, 1605-1612.

Pleiner, T., Bates, M., Trakhanov, S., Lee, C.-T., Schliep, J.E., Chug, H., Böhning, M., Stark, H., Urlaub, H., and Görlich, D. (2015). Nanobodies: site-specific labeling for superresolution imaging, rapid epitope-mapping and native protein complex isolation. Elife 4 .

Punjani, A., Rubinstein, J.L., Fleet, D.J., and Brubaker, M.A. (2017). cryoSPARC: algorithms for rapid unsupervised cryo-EM structure determination. Nat. Methods 14, 290-296.

Rousseau, A., and Bertolotti, A. (2016). An evolutionarily conserved pathway controls proteasome homeostasis. Nature 536, 184-189. 
Scazzari, M., Amm, I., and Wolf, D.H. (2015). Quality control of a cytoplasmic protein complex: Chaperone motors and the ubiquitin-proteasome system govern the fate of orphan fatty acid synthase subunit Fas2 of yeast. J. Biol. Chem. 290, 4677-4687.

Scheres, S.H.W. (2012). RELION: implementation of a Bayesian approach to cryo-EM structure determination. J. Struct. Biol. 180, 519-530.

Schindelin, J., Arganda-Carreras, I., Frise, E., Kaynig, V., Longair, M., Pietzsch, T., Preibisch, S., Rueden, C., Saalfeld, S., Schmid, B., et al. (2012). Fiji: an open-source platform for biological-image analysis. Nat. Methods 9, 676-682.

Schrader, J., Henneberg, F., Mata, R.A., Tittmann, K., Schneider, T.R., Stark, H., Bourenkov, G., and Chari, A. (2016). The inhibition mechanism of human 20 S proteasomes enables nextgeneration inhibitor design. Science 353, 594-598.

Schrödinger, L. (2015). The PyMOL Molecular Graphics System, Version 1.8.

Schweizer, E., and Hofmann, J. (2004). Microbial Type I Fatty Acid Synthases (FAS): Major Players in a Network of Cellular FAS Systems. Microbiol. Mol. Biol. Rev. 68, 501-517.

Shi, Y. (2014). A glimpse of structural biology through X-ray crystallography. Cell 159, 9951014.

Shiber, A., Döring, K., Friedrich, U., Klann, K., Merker, D., Zedan, M., Tippmann, F., Kramer, G., and Bukau, B. (2018). Cotranslational assembly of protein complexes in eukaryotes revealed by ribosome profiling. Nature.

Shpilka, T., Welter, E., Borovsky, N., Amar, N., Shimron, F., Peleg, Y., and Elazar, Z. (2015). Fatty acid synthase is preferentially degraded by autophagy upon nitrogen starvation in yeast. Proc. Natl. Acad. Sci. U. S. A. 112, 1434-1439.

Smith, S., Witkowski, A., and Joshi, A.K. (2003). Structural and functional organization of the animal fatty acid synthase. Prog. Lipid Res. 42, 289-317.

Stark, H., and Chari, A. (2016). Sample preparation of biological macromolecular assemblies for the determination of high-resolution structures by cryo-electron microscopy. Microscopy 65, 23-34. 
Steyaert, J., and Kobilka, B.K. (2011). Nanobody stabilization of G protein-coupled receptor conformational states. Curr. Opin. Struct. Biol. 21, 567-572.

Sumper, M., Oesterhelt, D., Riepertinger, C., and Lynen, F. (1969). [Synthesis of various carboxylic acids by the fatty acid synthetase multienzyme complex of yeast and the explanation for their structure]. Eur. J. Biochem. 10, 377-387.

Swanson, D., Block, R., and Mousa, S.A. (2012). Omega-3 Fatty Acids EPA and DHA: Health Benefits Throughout Life. Adv. Nutr. 3, 1-7.

Tang, L., Weissborn, A.C., and Kennedy, E.P. (1997). Domains of Escherichia coli acyl carrier protein important for membrane-derived-oligosaccharide biosynthesis. J. Bacteriol. 179, 3697-3705.

Tickle, I. Flensburg, C. Keller, P. Paciorek, W. Sharff, A. Vonrhein, C. Bricogne, G. (2018). STARANISO.

Tsumoto, K., Ejima, D., Senczuk, A.M., Kita, Y., and Arakawa, T. (2007). Effects of salts on protein-surface interactions: applications for column chromatography. J. Pharm. Sci. 96, 1677-1690.

Vagin, A., and Teplyakov, A. (2010). Molecular replacement with MOLREP. Acta Crystallogr. Sect. D Biol. Crystallogr. 66, 22-25.

Wakil, S.J., Stoops, J.K., and Joshi, V.C. (1983). Fatty Acid Synthesis and its Regulation. Annu. Rev. Biochem. 52, 537-579.

Wang, X.Y., Liang, Z.H., Huang, H.L., and Liang, W.X. (2011). Principles of ethics review on traditional medicine and the practice of institute review board in China. Chin. J. Integr. Med. 17, 631-634.

Wei, J., Zhang, Y., Yu, T.-Y., Sadre-Bazzaz, K., Rudolph, M.J., Amodeo, G.A., Symington, L.S., Walz, T., and Tong, L. (2016). A unified molecular mechanism for the regulation of acetyl-CoA carboxylase by phosphorylation. Cell Discov. 2, 16044.

Wenz, P. (2001). A downstream regulatory element located within the coding sequence mediates autoregulated expression of the yeast fatty acid synthase gene FAS2 by the FAS1 
gene product. Nucleic Acids Res. 29, 4625-4632.

White, S.W., Zheng, J., Zhang, Y.-M., and Rock, C.O. (2005). THE STRUCTURAL BIOLOGY OF TYPE II FATTY ACID BIOSYNTHESIS. Annu. Rev. Biochem. 74, 791-831.

Winn, M.D., Ballard, C.C., Cowtan, K.D., Dodson, E.J., Emsley, P., Evans, P.R., Keegan, R.M., Krissinel, E.B., Leslie, A.G.W., McCoy, A., et al. (2011). Overview of the CCP4 suite and current developments. Acta Crystallogr. Sect. D Biol. Crystallogr. 67, 235-242.

Yang, B., Wu, Y.J., Zhu, M., Fan, S.B., Lin, J., Zhang, K., Li, S., Chi, H., Li, Y.X., Chen, H.F., et al. (2012). Identification of cross-linked peptides from complex samples. Nat. Methods 9 , 904-906.

Zhang, K. (2016). Gctf: Real-time CTF determination and correction. J. Struct. Biol. 193, 112.

Zheng, S.Q., Palovcak, E., Armache, J.P., Verba, K.A., Cheng, Y., and Agard, D.A. (2017). MotionCor2: Anisotropic correction of beam-induced motion for improved cryo-electron microscopy. Nat. Methods 14, 331-332.

Zhou, Y.J., Buijs, N.A., Siewers, V., and Nielsen, J. (2014). Fatty Acid-Derived Biofuels and Chemicals Production in Saccharomyces cerevisiae. Front. Bioeng. Biotechnol. 2, 32.

Zivanov, J., Nakane, T., Forsberg, B.O., Kimanius, D., Hagen, W.J., Lindahl, E., and Scheres, S.H. (2018). New tools for automated high-resolution cryo-EM structure determination in RELION-3. Elife 7. 



\title{
Curriculum Vitae
}

\author{
Kashish Singh
}

Born $30^{\text {th }}$ August 1992 in Jammu, India

Address: Immanuel-Kant Str. 34, 37083 Goettingen

Phone: +49 (0) 17672619495

E-mail: ksingh2@gwdg.de

\section{University Education}

06/2015 - present Max Planck Institute for Biophysical Chemistry, Goettingen, Germany. Doctoral studies in the lab of Prof. Holger Stark.

PhD thesis: New sample preparation techniques of macromolecular complexes for high resolution structure determination using cryo$E M$

09/2013-03/2015 International Max Planck Research School in Molecular Biology, Georg-August University Goettingen. Masters in Molecular Biology (M.Sc)

Master thesis: Purification of Human chaperonin TRiC/CCT in the lab of Prof. Holger Stark.

06/2010-06/2013 Sri Venkateswara College, University of Delhi

Bachelor of Science with honors in Biochemistry

\section{High School Education}

04/1998 - 03/2010 Air Force Bal Bharti School, New Delhi 